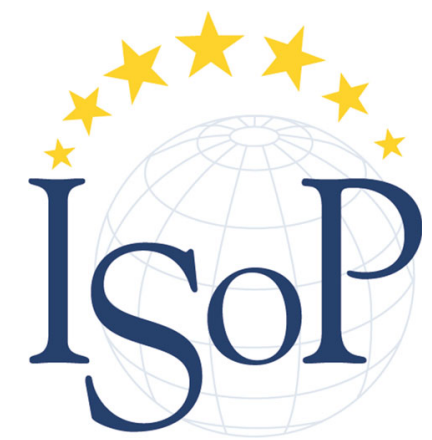

17th ISoP Annual Meeting "Pharmacovigilance in the 21st Century" Liverpool, UK 15-18 October, 2017

\title{
ABSTRACTS
}

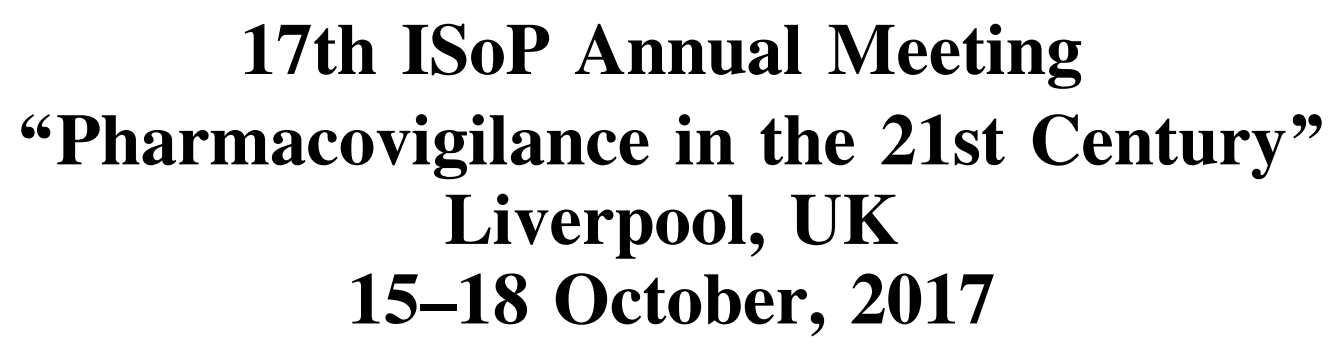




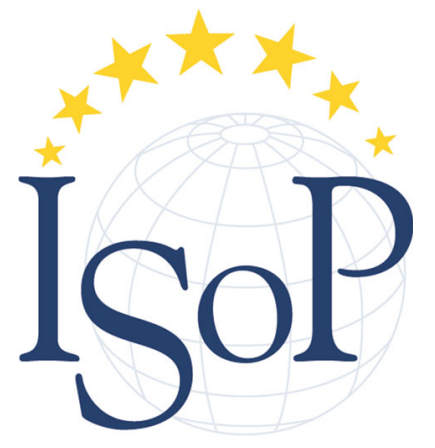

\section{INTERNATIONAL SOCIETY OF PHARMACOVIGILANCE}

The International Society of Pharmacovigilance (ISoP) is devoted to developing its activities on a worldwide basis towards supporting safer use of medicines in clinical practice.

ISoP aims to promote the use of all types of information and methodologies in providing optimal drug treatment for patients. The Society is not only for clinical pharmacologists, pharmaceutical industry representatives, epidemiologists and regulators, but also for practising clinicians, other healthcare professionals and anyone else who is interested in learning about better ways for patients to receive and use medicines safely.

\section{Countries where there are ISoP members:}

From Argentina to Vietnam, from countries in South-America to North-America, from Europe to Asia and Australia via Africa, we have members in all five continents.

"By becoming a member of ISoP, you will have the opportunity to share your knowledge and ideas and to contribute to improving pharmacovigilance activities worldwide."

Sten Olsson, President of the International Society of Pharmacovigilance

\section{ISoP Membership incentives include:}

- Biannual newsletters (ISoP Star)

- Training workshops

- Reduced fees for Annual Meeting and training courses

- Discounted online subscription to the Drug Safety journal

- Other offers/discounts on books

- Involvement in ISoP's Chapters and Special Interest Groups

For more information you can visit www.isoponline.org, the Society's official website

\section{International Society of Pharmacovigilance}

ISoP Secretariat Ltd

140 Emmanuel Road, London SW12 0HS, UK

Tel and Fax: +44 (0)20 32560027

administration@isoponline.org 


\title{
ISoP 2017 Local Organising Committee
}

Chair: Munir Pirmohamed

Ana Alfirevic

Andrew Bate

Dan Carr

Brian Edwards

Richard Fitzgerald

Neil French

Daniel Hawcutt

Simon Maskell

Christine Randall

Andrew Thompson

\section{ISoP 2017 Scientific Committee}

Chair: Hervé Le Louet (France)

Co-chair: Munir Pirmohamed (UK)

Luis Alesso (Argentina)

Raul Andrade (Spain)

Jeff Aronson (UK)

Joanne Barnes (New Zealand)

Chia-Yu Chu (Taiwan)

Jayesh Pandit (Kenya)

Peter Pitts (USA)

June Raine (UK)

Ian Wong (UK)

\section{ISoP 2017 Poster Prize Committee}

Chair: Jeffrey Aronson (UK)

Ana Alfirevic

Deirdre McCarthy

\section{ISoP Executive Committee and Advisory Board 2016-2019}

\author{
Sten Olsson, President (Sweden) \\ Ian Wong, Vice-President (UK) \\ Mira Harrison-Woolrych, Secretary General (New Zealand) \\ Jean-Christophe Delumeau, Treasurer (Singapore)
}

\section{Board Members}

Hilda Ampadu, Africa/ Education and Training Programme (Ghana)

Brian Edwards, Coordinator Chapters (UK)

Deirdre McCarthy, Coordinator Special Interest Groups (USA)

Jan Petracek, America/ Education and Training Programme (Czech Republic)

Phil Tregunno, Asia/ Education and Training Programme (UK)

Marco Tuccori, Europe/ Education and Training Programme (Italy)

Hervé Le Louet, Past-President (France)

\section{Disclaimer}

ISoP requests that a high standard of science is followed concerning publications and presentations at all its Annual Meetings and training courses. However, ISoP as a whole or its Advisory Board and Executive Committee (EC) or appointed Scientific Committees, or its members, do not take any responsibility for the completeness or correctness of data or references given by authors in publications and presentations at official scientific meetings. It is not within the remit of ISoP, the Advisory Board, the EC or Scientific Committees in particular, to seek clarification or detailed information from authors about data in submitted abstracts. Moreover, it is not within the scope of ISoP and its committees to monitor compliance with any legal obligations, e.g. reporting requirements or regulatory actions. 


\section{Challenges of Identifying Safety Signals in Clinical Trials}

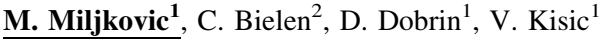 \\ ${ }^{1}$ PrimeVigilance, Belgrade, Serbia, ${ }^{2}$ PrimeVigilance, Zagreb, Croatia
}

Introduction: Clinical trials (CTs) conducted during product development programme are mostly designed to detect beneficial effects, while determining product safety is seldom selected as one of the primary outcomes. Pivotal studies identify common adverse events (AEs) related to the therapy and may help to identify signals for less common AEs [1].

Aim: To review methodological challenges and potential solutions in identifying safety signals from CT sources.

Methods: The guidelines and recommendations (EMA, FDA, CIOMS) related to signal detection/management and safety assessments in clinical trials [1-5] were used, and also systematic reviews focusing on product safety [6] published from 2005 to 2016

Results: Statistical power of CTs to detect the specific harm is limited either by recruitment of low-risk participants or by low intensity of determination of events. Study size and duration are limiting factors for detection of rare and long-term safety problems. Extrapolation of safety data to wider populations is difficult due to recruitment criteria that typically exclude risky patients. Signal detection in CTs should be focused on potential risks identified during non-clinical studies or with similar products. Quantitative signal detection is most useful on large pivotal study (late stage) or pooled studies, while qualitative methods remain the basis of signal detection in early stages. Randomised controlled trials (RCTs) are suited for evaluating outcomes that occur close to treatment initiation and have relatively high baseline incidence even in untreated populations, and that provide risk increase for a specific AE. RCTs offer unconfounded comparison between groups of patients where the only difference is the intervention. Randomization prevents the selection bias and ensures against the accidental bias. RCTs should include explicit pre-specified monitoring of pharmacologically predictable AEs and ensure adequate follow-up of withdrawn participants. Pooled analysis (with appropriate statistical method and optimal information size to avoid that the signal remains undetected) can provide reliable and valid comparative evaluation of drug safety.

Conclusions: Due to stated methodological limitations of CT, pooled analysis of CT datasets is important component in identifying signals in premarketing period. The major challenge for regulators in pharmacovigilance in the 21 st century is to make specific guideline for the signal management in clinical trial.

Further sources of information/References:

1. FDA. Guidance for Industry. Premarketing risk assessment, 2005

2. FDA. Guidance for Industry. Safety Assessment for IND Safety Reporting. Draft, 2015

3. Report of CIOMS Working Group VI. Management of Safety Information from Clinical Trials. Geneva, 2005

4. Report of CIOMS Working Group VIII. Practical Aspects of Signal Detection in Pharmacovigilance. Geneva, 2010

5. EMA. Guideline on good pharmacovigilance practices (GVP). Module IX-Signal management, 2012

6. Keisu M, Andersson TB. Drug-induced liver injury in humans: the case of ximelagatran. Handb Exp Pharmacol. 2010; (196): 407-18

\section{Statins and Diabetes: Are All Statins at Risk?}

J. Montastruc $^{1}$, F. Montastruc ${ }^{1}$, J. Benevent ${ }^{1}$, V. Rousseau ${ }^{1}$, L. Chebane $^{1}$, E. Bondon-Guitton ${ }^{1}$, G. Durrieu ${ }^{1}$, A. Sommet ${ }^{1}$

${ }^{1}$ Faculté De Médecine De Toulouse, Toulouse, France

Introduction: The association between diabetes and statins is well demonstrated, with, according to the studies, an increased risk for incident diabetes of $9-12 \%$ in general. Results also showed conflicting results according to the different statins, with, for example association with atorvastatin or rosuvastatin, but not with other statins, in the Takker's metaanalysis [1].

Aim: Since these results were obtained from clinical trials, we decided to investigate this safety signal in real conditions of life, using a pharmacovigilance database.

Methods: Using Vigibase ${ }^{\circledR}$, we performed a disproportionality analysis (case/non-case method) for the signal of diabetes with statins, including ICSRs until March 2017, only if age ( $\geq 18$ years) and gender were known. Cases were ICSRs with diabetes and non-cases all other ICSRs reports registered during the same period. Diabetes cases were defined as reports registered under the HLT MedDRA terms "diabetes mellitus" or "hyperglycemic conditions" in the SOC "Metabolism and Nutrition Disorders". Drug exposure to statins (atorvastatin, cerivastatin, fluvastatin, lovastatin, pitavastatin, pravastatin, rosuvastatin, simvastatin) was identified using the ATC code C10AA, i.e. HMG CoA reductase inhibitors defined as "suspected" or "concomitant". Strength of the link between statins' exposure and diabetes was quantified by crude Reporting Odds Ratio (ROR) with their 95\% CI. P threshold was 0.05 .

Results: Among the 9,372,588 ICSRs included, 13,071 involved the 8 studied statins plus diabetes. Most of the patients were women (69.3\%) with age mainly between 45 and 64 years $(57.0 \%)$. The 3 most frequently involved statins in the diabetes' ICSRs were atorvastatin $(62.4 \%)$ followed by simvastatin $(20.4 \%)$ and rosuvastatin $(11.7 \%)$. A signal was found between occurrence of diabetes and exposure to statins in general $[\mathrm{ROR}=4.29$ (4.21-4.38)] and all other statins in particular. The higher ROR value was found with atorvastatin [7.27 (7.10-7.45)] followed by rosuvastatin [3.02 (2.86-3.17)] and the lowest with cerivastatin [1.38 (1.07-1.78)]. Sensibility analyses performed investigating ROR with one statin after exclusion of cases registered with the other statins show similar results.

Conclusion: The present study found a clear signal of diabetes for statins in general and all the statins in particular, suggesting that the diabetogenic effect of statins is a class property. This is the first study investigating the problem of diabetes according to the different statins in the context of real world.

Further sources of information/References:

1. Thakker D, Nair S, Pagada A, et al. Statin use and the risk of developing diabetes: a network meta-analysis. Pharmacoepidemiol Drug Saf 2016; 25: 1131-49

\section{Impact of Pharmacovigilance and Evaluation Activities-A regulatory Perspective}

\section{R. Mouchantaf ${ }^{1}$, N. Comarova ${ }^{2}$, F. Bouterfa ${ }^{2}$}

${ }^{1}$ Health Canada, Ottawa, Ontario, Canada, ${ }^{2}$ University of Montreal, Montreal, Quebec, Canada 
Background: The past 50 years have witnessed an emergence of activities in the field of pharmacovigilance with an observed increase in post-market requirements. In view of the broad range of pharmacovigilance activities that are now at the disposal of the regulator, it is timely to analyse if such post-market processes are meeting their goals.

Aim: The current study evaluates the impact of certain pharmacovigilance activities with focus on signal detection and evaluation including the adoption of risk management planning (RMPs) in Canada. This is important given the increased focus on proactive signal detection and risk management.

Methods: (a) Signal detection activities internal to Health Canada from 2008 to 2015 were evaluated with scope limited to prescription pharmaceuticals. Signals were identified from scanning: Canada Vigilance database, manufacturer information, literature, foreign agencies, and other internal review activities. For each signal the source of we determined the source of data that gave rise to it and collected the signal recommendations. (b) For RMPs, an assessment was carried out to determine the extent to which RMPs have impacted Health Canada's ability to anticipate safety concerns. RMPs included in the submission for approval of a new active substance from 2007-2015 were screened for identification of preapproval safety concerns.

Results: Number of safety signals screened has steadily increased, in comparison number of signals validated has remained consistent $(\sim 30 /$ yr). In contrast, an increase was noted in activities related to review of submissions by the manufacturer (e.g., RMPs). The majority of the signals were detected from scanning foreign agencies $(34 \%)$ and literature $(21$ $\%)$. In parallel, through the review of RMPs post-market safety signals were identified in $66 \%$ of the cases. In relation to recommendations stemming from signal assessments, the majority were label changes (61 $\%)$ or routine monitoring (33\%) with a decline in additional activities recommended. Evidence from spontaneous reports contributed to over 65 $\%$ of the safety-related labelling changes. Analysis of domestic reports showed that the majority were reported by the manufacturer $(82 \%)$.

Conclusion: Introduction of RMPs has impacted a number of review activities and, provided the regulator with a structured approach for the detection and management of safety issues. Also, spontaneous reporting of adverse events continues to play a major role in regulatory decision making. Finally, assessing the effect of a regulatory activity has proven to be challenging given the multitude of determinants that may impact the outcome of an activity under study.

\section{Application of the Bleeding Academic Research Consortium (BARC) Definition for Bleeding Outcomes within a Post Authorization Safety Study}

\author{
M. Davies ${ }^{1,2}$, A. Evans ${ }^{1,2}$, S. Shakir ${ }^{1,2}$ \\ ${ }^{1}$ Drug Safety Research Unit, Southampton, UK, ${ }^{2}$ University \\ of Portsmouth, Portsmouth, UK
}

Background: In cardiovascular (CVS) clinical trials, bleeding endpoints are important as they help to define the benefit risk profile for antithrombotic (AT) agents and invasive procedures. It is acknowledged that there has been a high degree of heterogeneity between bleeding endpoint definitions within CVS trials. The Thrombolysis in Myocardial Infarction (TIMI) bleeding criteria have been most widely used in CVS trials, although this definition has its limitations. In 2010 the Bleeding Academic Research Consortium (BARC) proposed a clinical trial and/or registry bleeding endpoint definition, which included criteria relating to Coronary Artery Bypass Graft (CABG) procedures. The recommendation for researchers was to use the BARC bleeding endpoint definition in trials, even if in conjunction with other definitions. Bleeds reported in a Specialist Cohort Event Monitoring (SCEM) study on the oral anticoagulant rivaroxaban using real world data and conducted as part of Risk Management Plan, will be classified using both these definitions.

Objectives: To describe the methodological considerations of applying this definition to observational data.

Methods: Aim to collect data on a cohort of patients (pts) treated with rivaroxaban for the prevention of atherothrombotic events in pts who have had acute coronary syndrome, plus a contextual cohort of pts treated with standard anti-platelet therapy. Short-term (up to 12 weeks) safety will be assessed via quantification (risk and rate) of bleeding outcomes defined according to both BARC and TIMI. Recruitment Sep 2015-2018. TIMI and BARC criteria ascertained via a data capture form; hence each bleed will be classified according to both sets of criteria.

Results: To minimise misclassification, supplementary information will be used to validate the site and type of bleed, and collect further details relating to the outcome and management. All bleeds will be adjudicated by an expert and interim results will be published.

Conclusions: By systematically applying both TIMI and BARC definitions, we hope to gain better understanding of the type of bleeds reported in a cohort of AT users, associated risk factors and outcome details. We will also be able to compare and contrast definitions reported within both sets of criteria, and in relation to clinical trials results. We also aim to demonstrate how these definitions can be successfully applied to real world data.

\section{Drugs, Adverse Drug Reactions and PharmacoVigilance in the Adventures of Tintin}

\author{
J. L. Montastruc ${ }^{1}$, C. Damase-Michel ${ }^{1}$, I. Lacroix ${ }^{1}$, G. Durrieu ${ }^{1}$,

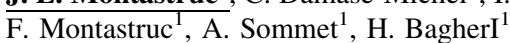

${ }^{1}$ Faculté De Médecine de Toulouse, Toulouse, France

Introduction: The Adventures of Tintin are a series of 24 comics' album, reporting the activities of the Belgian reporter Tintin, with his faithful dog Snowy (Milou in French), the brash and cynical Captain Haddock and the wacky and hearing-impaired Professor Calculus (Tournesol) as well as the incompetent detectives Thomson and Thompson (Dupont et Dupond) with the opera diva Bianca Castafiore.

Methods: We reviewed the references to drugs, Adverse Drug Reactions and PharmacoVigilance in the 24 albums.

Results: The most frequently found drug was chloroform cited in 6 books (Tintin in the Land of the Soviets, Tintin in America, The Blue Lotus, The Black Island, The Secret of Unicorn, Tintin and the Picaros...), followed by opium (Cigars of the Pharaoh, The Blue Lotus, The Crab with the Golden Claws) and quinine (used as antipyretic in Tintin in the Congo and Cigars of the Pharaoh). Other drugs were mentioned like camphor alcohol (unknown indication in Destination Moon), magnesia (as a gastric protective drug in Flight 714 to Sydney). Cocaïne was also found in Cigars of the Pharaoh and Land of Black Gold.

Other references include drugs to make alcohol unbearable (antabuse effect? in Tintin and the Picaros), the poison of madness (Cigars of the Pharaoh, The Blue Lotus) and the OX-2Z asphyxic gas (Tintin in Amer$i c a)$. In Tintin and the Alph-Art, there was a reference to medicine, Castafiore saying to the captain: "You must take your medicine" which in fact was a Loch Lomond whisky!

The most interesting part concerns pharmacovigilance with acetylsalicylic acid as described in 2 albums. First, in Land of Black Gold, Thomson and Thompson, suffering from headache, took gasoline which was presented as aspirin tablets 
(falsified drug?). They suffered from two adverse drug reactions (ADRs): first, an immediate "labeled" ADR (nausea, vomiting) and second, an "unlabeled" one, a marked hypertrichosis (whiskers, hair) that was "serious" leading the detectives to Wadeshah hospital, where a nurse found the cases "extra-ordinary" and asked to the doctor to "come quickly". The doctor was very surprising. Unfortunately, in 1947 , there was none pharmacovigilance center! Later, in Explorers on the Moon, Thomson and Thompson took again aspirin with recurrence of hypertrichosis. According to WHO-UMC scale, we evaluated the ADR's causality as "certain" due to a positive rechallenge.

Conclusion: The present analysis found several pharmacological and pharmacovigilance bases in The Adventures of Tintin. These comics can be useful to teach pharmacology and pharmacovigilance.

\section{Olpadronate retrospective compilation of adverse events: Determination of dose of exposition (DOE) until the first adverse event occurrence}

\section{Guadalupe García Darderes ${ }^{1}$, C. Gómez Acotto ${ }^{2}$, L. DAlessio ${ }^{3}$, E. Roldán}

${ }^{1}$ Gador S.A., Darwin, Argentina, ${ }^{2}$ University Maimonides, PhosphoCalcium Department, Hidalgo, Argentina, ${ }^{3}$ Universidad de Buenos Aires, Paraguay, Argentina

Introduction: Bisphosphonates such as OPD are used in the treatment of skeletal disorders such as Pagets disease and osteoporosis. But new indications are under development, therefore safe schedules are on demand. As intermittent schedules may be an option, we hereby report a compilation of OPD adverse effects aiming to estimate a specific exposition free of adverse events, by either I.V. or oral routes.

Methods: Adverse events were compiled retrospectively from a series of patients having Pagets bone disease and treated with OPD at a university hospital in which the drug was authorized. Doses ranged from 4-8 mg/day I.V., and/or 100-200 mg/day orally. The OPD exposition until the first event (DOE) was calculated using the formula: ( [daily dose multiplied by treatment duration until first adverse event reported] divided by body weight). The outcome was further divided by the bioavailability rate 0.035 when the administration had been orally. Students $\mathrm{T}$ test was used for the statistical analysis.

Results: One hundred patients, aged $60.8 \pm 10.6$ years ( 39 females) were included. More frequent adverse events were hypocalcemia (59\% total sample), flu like (23\%), leukopenia (16\%), fever (15\%), phlebitis (8\%), headache $(4 \%)$, somatic pain $(7 \%)$, hypersensitivity (2\%), and dizziness (2\%). DOE after I.V. administration was significantly lower than after oral route for hypocalcemia $(0.20 \mathrm{vs} 0.33 \mathrm{mg} / \mathrm{Kg} ; \mathrm{p}<0.05)$ and for flu like $(0.09$ vs. $0.17 \mathrm{mg} / \mathrm{Kg} ; \mathrm{p}<0.05)$ and no significant differences in oral versus I.V. DOE ( 0.23 vs. $0.19 \mathrm{mg} / \mathrm{Kg}$ ) were observed for leukopenia $(\mathrm{p}>0.05)$.

Conclusion: The estimation of OPD DOE may help in the design of comfortable safe therapeutic schemes by providing intermittent oral and / or I.V. schedules best tolerated.

\section{Parents' Perception and Practices Regarding Immunization: A Cross Sectional Investigation}

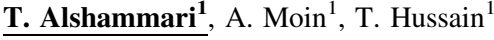 \\ ${ }^{1}$ University of Hail, Hail, Saudi Arabia}

Introduction: Vaccines represent one of the greatest achievements of science and medicine in the battle against disease. It remains one of the most economical public health policies to decrease disease and death associated with vaccine preventable diseases. Despite thorough scientific proof that vaccinations are amongst the safest and most cost-effective public health measures, accomplishment and maintenance of high levels of immunization coverage has remained a challenge [1-3].

Aim: The present investigation attempts to understand parents' perception and practices regarding immunization.

Methods: The survey instrument was a questionnaire containing 18 questions in three domains namely demographic characteristics, parent awareness and practices aligned to the set objectives. Randomly selected parents were interviewed according to the questionnaire and the response was noted. A cross sectional study design was used to carry out the survey with a sample size of 467 parents in public places and hospitals at Hail and other parts of Saudi Arabia.

Results: Results indicate Female $(54.5 \%, \mathrm{n}=255)$ parents were $9 \%$ more than the males $(45.5 \%, \mathrm{n}=212)$, more than $75 \%(\mathrm{n}=357)$ of the parents were in the age group of $18-55$ years, and approximately $94.1 \%(n=439)$ parents were educated. The vast majority of the population $368(78.9 \%)$ was aware of vaccination/immunization \& had vaccinated their kids. However, $46.8 \%(n=219)$ of the total surveyed population was unaware of specific immunization programs in Saudi Arabia and 45.6\% $(\mathrm{n}=213)$. Surprisingly one tenth $(11.6 \%, \mathrm{n}=54)$ of the respondents were aware of the perceived popular $\mathrm{MoH}$ mobile remainder. $91.1 \%$ of the population expressed that they never faced any problems during or after vaccination. About $86.2 \%(n=403)$ respondents strongly agreed that their children had received all the vaccines that are recommended as per the ministry of health, KSA.

Conclusions: From the investigations and obtained results, it clearly indicated that the parents are well aware of immunization and its relevance in child's health. Additionally, the necessary practices of immunization were adhered, which is in the interest of the successful immunization program and for betterment of the society.

Further sources of information/References

1. Fiore AE, Bridges CB, Cox NJ. Seasonal influenza vaccines. Curr Top Microbiol Immunol 2009; 333: 43-82

2. Chang Y, Brewer NT, Rinas AC, Schmitt K, Smith JS. Evaluating the impact of human papillomavirus vaccines. Vaccine 2009; 27: 4355-62

3. Allison K, Katherine LV, Glen N, Michelle B, Sarah L. Confidence about vaccines in the United States: Understanding parents' perceptions. Health Affairs 2011; 30: 1151-59

\section{A way to classify the prescriptive cascades}

\author{
$\underline{\text { M. Ponte }}{ }^{1}$, A. Serra ${ }^{1}$
}

${ }^{1}$ Pontificia Universidad Catolica Buenos Aires, Ciudad Autonoma De Buenos Aires, Argentina

Background: A prescriptive cascade (PC) is the counterpart of current pharmacology. New treatments require greater knowledge and skills that are not always accompanied by adequate professional training. Thus, by mistake or omission it falls into such cascade. Previously we have developed a score and an algorithm for its measurement. However, some points remain to be defined, basically the cascade types.

Aim: the aim of this communication is to classify the types of PC.

Methods: Using the tertiary care Hospital Argerich (Autonomous City of Buenos Aires) pharmacovigilance database.

Results: from a total ADR ( $\mathrm{n}=2990$ cases), we detected a total of 398 PC $(13.31 \%)$. According to their outcome we propose three types: 
Mandatory, because it is necessary to execute it as part of an accepted treatment, for example, the use of antiemetics after the antineoplastic treatment $(\mathrm{n}=218$ cases; 55\%). Absolutely avoidable, because its recognition and prevention is the necessary condition to avoid major dangerous ADRs, for example, the use of anticonvulsants to treat seizures induced by beta-lactams, since the former can cause serious dermatologic reactions ( $\mathrm{n}=155$ cases; 39\%). Intermediate, because the prevention and cutoff are not defined, for example, the use of vitamins against statin induced asthenia $(\mathrm{n}=25$ cases; $6 \%)$.

Conclusion: Majority of PC are mandatory but a great number of this are avoidable. Some of mandatory PC could be prompt detected to decrease severity of PC.

Discussion: It is important to trainee all health community to identify this problem and avoid the preventable ones.

\section{Pevalence of Prescriptive Cascades}

M. Ponte ${ }^{1}$, A. Serra ${ }^{1}$, M. Frojan ${ }^{1}$, L.N. Noferi ${ }^{1}$, J.M. Fernandez Acuña $^{1}$, F. Paso ${ }^{1}$, J.F. Vignale ${ }^{1}$, E.J. Tosi ${ }^{1}$, D.C. Manzano ${ }^{1}$

\section{${ }^{I}$ Pontificia Universidad Catolica Buenos Aires, Ciudad Autonoma De Buenos Aires, Argentina}

Background: The prescriptive cascade (PC), a term coined by Paula Rochon in 1998, refers to the use of a new drug to treat an adverse drug reaction that was not appropriately noticed and diagnosed. Recently, we have defined a score and an algorithm to determine its existence and the possibility of preventing it.

Aim: Here we described its prevalence in a tertiary care Hospital in the Autonomous City of Buenos Aires during the period 2010-2015.

Method: We used the Argerich Hospital pharmacovigilance database ( $\mathrm{n}=2990$ cases) and we applied the $a d$ hoc score (a cutoff value of more than 4) (table 1). We were able to identify a total of 398 PC $(13.31 \%)$ during the considered period. Classified according to apparatus or system PC were: CNS $(\mathrm{n}=19)$; Endocrine $(\mathrm{n}=40)$; Digestive $(\mathrm{n}=103)$; Cardiovascular $(\mathrm{n}=12)$; and locomotor $(\mathrm{n}=6)$. With the algorithm, we determined that $95 \%$ of them were preventable.

Conclusion/discussion: In conclusion, we believe that the tools developed are of value in terms of the identification of the prescriptive cascades, but it takes a lot of training of the personnel to think about PC in order to avoid them.

\section{Knowledge and attitude of health care professionals toward adverse drug reactions reporting at King Saud Medical City}

\author{
S. Ali ${ }^{1}$, K. Moinuddin, S. Salem, A. Al-Aqqad, M. Al-Dossari, \\ A. Ananzeh, J. Baqar

\section{${ }^{1}$ Ministry of Health, Riyadh, Saudi Arabia}

Introduction: Health care professionals across the globe are obligated to report adverse drug reactions (ADRs). The reporting of ADRs is itself regarded as an important source of information for health care professionals and the quality of collected ADR reports mostly depend on their reported data. [1-2] This is the first study that explored the knowledge and attitude of health care professionals (HCPs) regarding ADR reporting in a large tertiary care hospital in the Kingdom of Saudi Arabia.
Aim: The aim of this study was to assess the understanding of health care professional's awareness regarding ADRs reporting and their perspective towards its reporting which address the factors of underreporting, as well. Methods: A cross-sectional study using an anonymous questionnaire was conducted over a period of 3 months (September 2016-November 2016) at King Saud Medical City (KSMC), Riyadh. Descriptive statistics were applied to the categorical variables and represented as frequency and percentages. The influence of gender and nationality differences on the responses of knowledge and attitude questions was also evaluated by performing chi-square test.

Results: Total 399 forms received and responding females (86.7\%) were more than males. About $34.3 \%$ responders were belong to the age group 26-30 years and $76.2 \%$ were international employees. Only $14.8 \%$ knew the term ADR but $55.1 \%$ responders reported the ADRs during their practice. Interestingly $70.2 \%$ answered that all health care professionals are responsible to report ADRs and $92 \%$ said all types of ADRs should be reported. The $93.8 \%$ responders agreed that ADR reporting should be mandatory for health care professionals and $94.5 \%$ agreed that it improves the patient safety. Only $17.8 \%$ agreed that ADR reporting is not important for health care system and $58.4 \%$ agreed that legal liability issues affect the ADR reporting.

Conclusions: It was found that health care professionals are less aware about the term ADR but they know the importance of ADR reporting which is directly related to improving the patients' safety and health care system. There is a clear need of PV awareness programs for health care professionals and a large scale multicenter study would help to formulate a strategy to encourage ADR reporting by health care professionals.

\section{Further sources of information/References}

1. The Importance of pharmacovigilance Safety monitoring of medicinal products. WHO Lib Catalog. 2002. Available from: http://apps.who. int/iris/bitstream/10665/42493/1/a75646.pdf (Accessed 27 Feb 2016)

2. Reporting adverse drug reactions: definitions of terms and criteria for their use. Geneva, Council for International Organizations of Medical Sciences (CIOMS), 1999

\section{Direct oral anticoagulants: are they safer than vitamin $\mathrm{k}$ antagonists?}

\author{
M. Baumberger ${ }^{1}$, O. Hugli ${ }^{2}$, T. Buclin ${ }^{3}$, F. Livio $^{3}$
}

${ }^{1}$ Medical School, Faculté de Biologie et de Médecine, University of Lausanne, Lausanne, Switzerland, ${ }^{2}$ Emergency Department, Centre Hospitalier Universitaire Vaudois, Lausanne, Switzerland, ${ }^{3}$ Division of Clinical Pharmacology, Centre Hospitalier Universitaire Vaudois, Lausanne, Switzerland

Introduction: A significant fraction of the Swiss population is treated with anticoagulants. Bleeding complications are frequent, counting for about $10 \%$ of adverse drug reaction-related hospitalizations. After decades of exclusive use of vitamin $\mathrm{K}$ antagonists (VKA), direct oral anticoagulants (DOACs) are now available and their use is increasing. According to the published randomized controlled trials, DOACs are at least as effective as VKA, but might be safer.

Objectives: This study aimed to evaluate whether DOACs are safer than VKA by comparing their respective consumption with the number of hospitalizations for bleeding complications in an emergency department over the past years.

Methods: We retrospectively collected data from all patients treated with DOACs or VKA who were admitted to the Emergencies of CHUV for bleeding with externalization (any site) between 2011 and 2015. The 
severity of these hemorrhages was graded. Patient characteristics were recorded. The consumption data of DOACs and VKA were obtained from Interpharma and The Cooperative of the Swiss Pharmacists for the years 2011 to 2015. Spontaneous reporting of bleeding events under DOACs and VKA to Swissmedic was extracted from the Vigilyze database.

Results: A total of 779 admissions for bleeding events were recorded, among which 250 in patients treated with DOACs or VKA. Only 15 cases were associated with DOACs and were compared with a sub-sample of 50 among the 235 cases of the VKA group. Patients in the VKA group were slightly older (79.3 vs 76.4 years; NS), with higher comorbidity Charlson scores (2.16 vs 1.46 ; NS), and lower renal function ( $44 \mathrm{vs} 57 \mathrm{ml} / \mathrm{min} /$ $1.73 \mathrm{~m}^{2}$ GFR; $p=0.006$ ). They had more often drug interactions (pharmacokinetic $: p=0.008$; pharmacodynamic $: p=0.004)$. The bleeding events did not differ significantly in their location, severity and management. The DOACs market share is growing with a number of DOACs units sold outpacing the VKA in 2015. The number of spontaneous reporting of DOACs-associated bleeding events to Swissmedic is higher than that of VKA since 2013.

Conclusion: The results tend to confirm a better safety of DOACs compared to VKA. However, VKA are prescribed to patients in less good condition and thus more at risk of bleeding, which may accentuate the difference in incidence of bleeding events. The over-representation of DOACs in the Swissmedic database might be due to reporting biases.

\section{Adverse drug reactions as a cause of admission to intensive care unit: a 4-months prospective cohort study}

\author{
V. Berthaut ${ }^{1}$, D. Renard ${ }^{2}$, C. Manzon ${ }^{3}$, M.D. Schaller ${ }^{3}$,

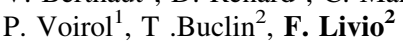

${ }^{1}$ Pharmacy, Centre Hospitalier Universitaire Vaudois, Lausanne, Switzerland, ${ }^{2}$ Division of Clinical Pharmacology, Centre Hospitalier Universitaire Vaudois, Lausanne, Switzerland, ${ }^{3}$ Intensive Care Unit, Centre Hospitalier Universitaire Vaudois, Lausanne, Switzerland

Introduction: Adverse drug reactions (ADR) are a significant cause of hospitalization, including in intensive care unit (ICU).

Aim: The aim of the present study was to assess the frequency and preventability of ADR-related admissions to ICU.

Method: Design: prospective 4-months observational study. Patients: all adult patients admitted to the Centre Hospitalier Universitaire Vaudois ICU between February and June 2015. Data collection: patient's age and gender, current drugs, Simplified Acute Physiology Score II (SAPS II), length of stay and clinical outcome. The causality between drugs and organ failure at admission was assessed for all patients by the principal investigator and two clinical pharmacologists using a standardized method. Drug abuses and suicide attempts were excluded. ADR preventability was assessed with standardized criteria. ICU patients admitted for ADR were compared to all others.

Results: A total of 615 admissions was recorded during the 4-months study period. Of these, 55 (8.9\%) admissions were ADR-related. A total of 18 ADR (33\%) was deemed preventable. Most frequent ADR (by affected organ) were infections $(36 \%, \mathrm{n}=20)$, CNS disorders $(18 \%, \mathrm{n}=10)$ and cardiac disorders $(13 \%, \mathrm{n}=7)$. Bleeding, any site, was common $(\mathrm{n}=14)$. Immunosuppressants, antithrombotics and beta blockers were the most frequent classes of drugs involved. Compared to control patients, those with ADR were more polymedicated (69\% vs 20\%), had higher SAPS II scores (48 vs 41 ) and hospital mortality ( $29 \%$ vs $13 \%)$. The mean length of ICU stay was 5 to 6 days in both groups. Only 1 out of those 55 ADR cases was spontaneously reported to the pharmacovigilance officer. Conclusion: ADR represent a frequent cause of ICU admission; frail polymedicated patients are at higher risk. A third of these ADR meets criteria of preventability. Better training in pharmacotherapy and awareness of drug-related risks among health professionals are important to limit the heavy human and financial burden associated with ADR.

\section{An MCEM-MTL Framework for Drug Safety Signal Filtering and Detection in Spontaneous Reporting Systems}

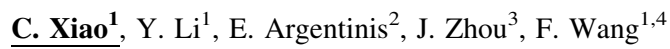

${ }^{1}$ Center for Computational Health, IBM T.J. Watson Research Center, Yorktown Heights, USA, ${ }^{2}$ Watson Health, Life Sciences Division, New York, USA, ${ }^{3}$ Computer Science and Engineering, Michigan State University, East Lansing, USA, ${ }^{4}$ Health Policy and Research, Weill Cornell Medical School, Cornell University, New York, USA

Introduction: Spontaneous reporting systems (SRS) serves as the cornerstone for post-marketing drug surveillance. A series of disproportionality analysis methods (DAMs) were developed to detect safety signals based on the SRS. Multi-item Gamma Poisson Shrinker (MGPS) is one of the most reliable DAMs and is in routine use by the FDA [1]. MGPS solves the sampling variance issue faced by other DAMs, but does not solve the confounding challenge induced by concomitant drugs [2].

Aim: Propose a computational framework that attempts to remove confounding by concomitant drug and improve signal detection performance. Methods: We proposed an integrated Monte Carlo Expectation-Maximization (MCEM) framework that leverages signal measures generated by MGPS. In addition to inheriting the benefits of MGPS, it has the following characteristics: (1) it filters out some confounders induced by concomitant drugs and consequently generates an improved count of " "in the $2 \times 2$ contingency table used in computing MGPS. This is achieved by assigning each ADR with one drug in the record as its major cause where the drug was determined via Monte Carlo sampling based on the probability proportional to risks. (2) it employs multitask learning with a Gaussian model to allow for related signals to borrow statistical support from one another. We performed experiments using the de-duplicated and standardized (e.g. drug names mapped to RxNorm concepts and ADR outcomes mapped to MedDRA concepts) FDA adverse event reporting system (FAERS) from 2006 to 2014 [3], and evaluated the results using a validated reference standard created by the Observational Medical Outcomes Partnership (OMOP) involving four serious adverse drug reactions (ADRs) - acute myocardial infarction (AMI), acute renal failure (ARF), acute liver injury (ALI), and upper gastrointestinal bleeding (GIB) [4]. By eliminating the test cases that were not included in the dataset, it resulted in 156 positive cases and 212 negative cases.

Results: The proposed framework achieved an AUC of 0.81 compared with an AUC of 0.77 produced by MGPS based on the reference standard. The evaluation based on each of the four ADR sets of test cases showed that the proposed framework achieves better AUC for three of four ADRs except for ALI.

Conclusions: This is the first of its kind that explicitly deals with concomitant drug confounders and leverages the advantages of the MGPS using an integrated MCEM scheme via a multitask learning (MTL) approach. Evaluation on real-world data demonstrates the promising utility and efficacy of the proposed framework. 


\section{Further sources of information/References}

1. DuMouchel W. Bayesian data mining in large frequency tables, with an application to the FDA spontaneousreporting system. Am Stat 1999;53:177-90

2. Harpaz R, DuMouchel W, Shah N, Madigan D, Ryan P, Friedman C. Novel data-mining methodologies foradverse drug event discovery and analysis. Clin Pharmacol Ther 2012;91:1010-21

3. Banda JM, Evans L, Vanguri RS, Tatonetti NP, Ryan PB, Shah NH. A curated and standardized adverse drug event resource to accelerate drug safety research. Scientific data 2016;3

4. Ryan PB, Schuemie MJ, Welebob E, Duke J, Valentine S, Hartzema AG. Defining a reference set to supportmethodological research in drug safety. Drug Saf 2013;36:33-47

\section{Alternative facts: why patient safety is under threat and what we can do about it}

\author{
B. Hugman ${ }^{1}$ \\ ${ }^{1}$ Uppsala Monitoring Centre, Uppsala, Sweden
}

Background: Scientists, experts and scientific evidence are increasingly under attack in public policy and popular discourse. While individual health practitioners remain respected by many, there is a growing climate of skepticism and denial in relation to public and personal health issues (vaccination, obesity, opioid abuse, for example); confusion and uncertainty about controversial issues (GMOs, climate change, nuclear power, pesticides, dietary sugar and fat, amongst many); and a strong drift towards irrationality (inter alia, ignorance or denial of evidence, dietary and lifestyle fads, alternative explanations and therapies (homeopathy, herbals), and belief-based solutions). These pose a major threat to the future impact and effectiveness of science and scientific thinking as a whole and for pharmacovigilance and patient safety, among many other fields vital to human welfare.

Objectives: This presentation will review some of the current trends that are threatening public respect for evidence and understanding of medicinal safety issues. This will lead to identification of some paths that may help science begin to recover ground lost in the recent past, along with some specific proposals for pharmacovigilance and patient safety.

Methods: The work behind this presentation is part of a larger project examining how medicines information can recover from its failure to deliver full benefits in an increasingly complex political, social and philosophical context.

Discussion: One of the missing elements in scientific communication is an analysis of and response to the complexities and influences of the political and social context in which it takes place. Evidence about specific issues must be communicated in ways that not only make it clear and transparent, but also acknowledge and manage the multiple extraneous obstacles that stand in the way of its visibility and acceptability. Recovery requires not only specific focused action within each of the multiple branches of science, but also a large, radical coalition, like that symbolically manifested in the March for Science.

Conclusion: Contemporary events suggest that the rise of populism will continue, with the consequence, among many, that respect for experts, evidence and rationality will continue to decline in the years ahead unless strong, imaginative measures are taken soon. The presentation will end with suggestions about some novel approaches that could be taken to mitigate the problems and that could benefit pharmacovigilance and patient safety.
22 A change point analysis to study the reporting pattern of ADRs after drug substitution

\author{
$\underline{\text { F. Van Hunsel }}{ }^{1}$, L. Pelzer ${ }^{1}$, A. Kant ${ }^{1}$ \\ ${ }^{I}$ Netherlands Pharmacovigilance Centre Lareb, 's Hertogenbosch, \\ the Netherlands
}

Introduction: The Netherlands Pharmacovigilance Centre Lareb regularly receives reports of adverse drug reactions (ADRs) after patients' brand of drugs have been changed (generic substitution). Often, these reports also mention a reduced drug efficacy. Usually these switches between brands of drugs are a result of the Preference Policy of Dutch Health Insurance companies, but drug shortages of a particular brand can also play a role. Aim: To identify for which drugs ADRs related to generic drug substitution were reported and to study the usability of a change point analysis (CPA) to detect meaningful changes in the reporting pattern over time. Methods: Reports from 2006-2016 containing the MedDRA ${ }^{\circledR}$ LLT 'Therapeutic response unexpected with drug substitution' were selected. For the drugs (ATC-7) that were reported most often ( $>25$ reports) a change point analysis (CPA) was used to get insight in the pattern of reports on drug substitution over time. With the CPA the reports of a specific association are counted per time period, and changes in the mean counts per time period can be detected by different methods (open-source software R) [1]. The reports were counted per week, and the Pruned Exact Linear Time (PELT) method with the 'Modified Bayesian Information Criterion (MBIC)' penalty was used to detect changes in the mean. The results of the CPA were compared with a clinical assessment of the reports by a trained assessor.

Results: Lareb received 2667 reports on ADRs related to drug substitution. There were 20 drugs with $>25$ reports on drug substitution. 12 Changepoints were detected for 5 drugs, and for 15 drugs there was no change point. Levothyroxine was the drug with the most ADRs $(\mathrm{N}=295)$ reported after drug substitution and with the most change points $(n=4)$. Most often a statistical 'change point' coincided with the action that Lareb had undertaken on the basis of case-by-case analyses. For 2 drugs (methotrexate and rivastigmine) a total of 3 signals were issued, while no statistical change point was detected.

Conclusions: The CPA can be a useful tool to study pattern changes. However, lack of a statistical change in the reporting pattern does not necessarily rule out that problems due to substitution did arise. This is also due to the chosen sensitivity of the CPA method, which prevents too many false-positive signals. Clinical assessment of the cases remains indispensable.

Further sources of information/References

1. Killick R, Eckley I. Changepoint: an R package for changepoint analysis. J Stat Softw 2014;58:1-19

\section{The Contribution of Direct Patient Reports to Drug Safety Signals in the Netherlands from 2010 to 2015}

\author{
F. Van Hunsel ${ }^{1}$, L. Harmark ${ }^{1}$ \\ ${ }^{1}$ Netherlands Pharmacovigilance Centre Lareb, 's Hertogenbosch, \\ the Netherlands
}

Introduction: Direct patient reporting can enrich pharmacovigilance and the Netherlands Pharmacovigilance Centre Lareb has favorable experiences with this relatively new reporting group [1-3]. The number of 
reports from patients to Lareb grew from 173 reports in 2003 to 8010 reports in 2015, while the number of healthcare professional (HCP) reports has remained stable. In the past few years, since the 2012 pharmacovigilance legislation, Lareb has also widened the scope of their drug safety signals, including ADRs related to overdose, abuse, off-label use, misuse and medication errors. With this increasing number of patient reports and a broader focus in signal detection, we re-analysed [4] the contribution of patient reporting to signal detection.

Aim: We aimed to study the current contribution of patient reports to disseminated signals to the Dutch Medicines Evaluation Board and in addition to determine if there are certain types of signals where patient reports add a distinct contribution compared to HCP and Marketing Authorization Holders (MAH) reports.

Methods: All signals from 2010 until 2015 were included. First, we investigated how many patient reports were present in the signals and the characteristics of these reports compared to $\mathrm{HCP}$ and $\mathrm{MAH}$ reports.

In addition to source, the analysis included ATC-code of the drug, MedDRA $^{\circledR}$ System Organ Class and Preferred Term for the ADR, seriousness of the ADR, and seven other factors like reports on Over-TheCounter medication and how often an ADR listed in the Important medical event terms list was present.

Secondly, we determined the proportion of reports submitted by the individual groups to signals, in a cross-sectional manner.

Results: 150 signals were included, including 1691 ADR reports. Our results show that $26.3 \%$ of all ADR reports in Dutch drug safety signals were reported by patients. $30.5 \%$ of the patient reports in the signals contained one or more terms listed as Important Medical Events. The proportion of reports by patients which were included the signals was 2 and $3.9 \%$ for HCP reports and $0.2 \%$ for MAH reports.

Conclusions: Patients had an important contribution to signals overall, but especially for ADRs related to generic drug substitution and Psychiatric ADRs.

Further sources of information/References

1. van Hunsel F, Härmark L, Pal S, Olsson S, van Grootheest K. Experiences with adverse drug reaction reporting by patients: an 11-country survey. Drug Saf 2012;35:45-60

2. Härmark L, van Hunsel F, Grundmark B. ADR Reporting by the General Public: Lessons Learnt from the Dutch and Swedish Systems. Drug Saf 2015;38:337-47

3. Rolfes L, van Hunsel FPAM, van der Linden, Taxis $\mathrm{K}$, van Puijenbroek EP. The quality of clinical information in adverse drug reaction reports by patients and healthcare professionals; a retrospective comparative analysis. Drug Saf 2017 (accepted for publication)

4. van Hunsel F, Talsma A, van Puijenbroek E, de Jong-van den Berg L, van Grootheest $\mathrm{K}$. The proportion of patient reports of suspected ADRs to signal detection in the Netherlands: case-control study. Pharmacoepidemiol Drug Saf 2011;20:286-91

\section{Awareness Survey on Pharmacovigilance: Design of an online program for Argentinean health care professionals}

\section{Guadalupe García Darderes ${ }^{1}$, J. Carlos Gallo ${ }^{2}$, E. Roldan ${ }^{1}$ \\ ${ }^{1}$ Gador S.A., Darwin, Argentina, ${ }^{2}$ Hospital Alvarez, Dr. J.F. Aranguren, Argentina}

Introduction: Argentinean National Regulatory Health Authority became official member of the WHO Program for International Drug Monitoring in
1994. However, the number of adverse drugs reactions (ADRs) reported is still very low. Programs in healthcare professionals (HCP) in other countries showed that after surveys the knowledge and awareness about pharmacovigilance reporting improved, and the number of reports increase (1-4). Aim: The aim of this study is to investigate the level of knowledge about pharmacovigilance and the activity of reporting an adverse event among local HCP, including dentists. At the same time, the survey will be used as an awareness tool. We hereby report on the study design.

Methods: A cross-sectional survey is being planned, using a validated selfadministered questionnaire. The questionnaires will be distributed to $\sim 10.000$ HCP from an own database, including general practitioners, pharmacists and dentists. The survey consists of questions related to adverse drug reactions and the reporting behavior of health care professionals in ordinary practice. Questions were chosen based on daily practice situations and relaxed responses. The survey form will be distributed by on-line responsive tool. The collected data will be then analyzed descriptively and results will be presented as mean and ranges, and \% of total answers. The survey will have two sections, first one collecting the number of respondents, and the second one clustering the answered questions.

Results: A form with a set of 15 questions and their correspondent multiple choice options for answering was designed. Basically, question focus on daily practice load, reporting situations, reporting behaviors, and to whom the ADRs are usually reported were chosen for this survey. The online form allows easy answering, estimated in 3-5 min. After reporting opinions, the participants will receive a feedback about their reporting role in practice. These materials will be exposed to allow discussions. Early example of results will be included upon availability.

Conclusions: The selected set of questions to be employed in a survey provides a simple way of increasing the awareness of health professional on their reporting role of ADRs. Besides, once analyzed it will allow to measure the awareness status of a given population, in this case health professionals living in Argentina.

Further sources of information/References:

1. Dorji C, Tragulpiankit P, Riewpainboon A, et al. Knowledge of adverse drug reaction among healthcare professionals in Buthan: A cross-sectional survey. Drug Saf 2016; 39: 1239-50

2. Lepakhin VK. Safety of Medicines: a guide to detecting and reporting adverse drug reactions. WHO/EDM/QMS/2002.2.Geneva: World Health Organization

3. Uppsala Monitoring Centre. Safety Monitoring of medicinal products. Uppsala SUMC; 2000

4. Bordet R, Gautier S, Le Louet H, Dupuis B, Caron J. Analysis of the direct cost of adverse drug reactions in hospitalized patients. Eur $\mathbf{J}$ Clin Pharmacol. 2001; 56:935-41

\section{Gender Differences in Reported Adverse Drug Reactions of SSRIs}

\author{
C. Ekhart ${ }^{1}, \underline{\text { F. van Hunsel }}{ }^{1}$, E. van Puijenbroek ${ }^{1,2}$ \\ ${ }^{I}$ Netherlands Pharmacovigilance Centre Lareb, 's Hertogenbosch, \\ the Netherlands, ${ }^{2}$ Dept. of Pharmacotherapy, Epidemiology \\ and Economics, University of Groningen, Groningen, the Netherlands
}

Background: Adverse drug reactions (ADRs) occur in approximately $3-5 \%$ of patients taking a drug. It appears that women experience ADRs more often than men [1,2]. A study by Zopf et al showed that female gender was a risk factor for encountering ADRs [OR 1.6, 95\% CI 1.3-1.9] and dose-related ADRs were the dominant type in female subjects. The 
reasons for this increased risk are not entirely clear but may include gender-related differences in pharmacodynamics, pharmacokinetics and physical differences (body-water space, muscle mass, organ blood flow, organ function).

Selective serotonin reuptake inhibitors (SSRIs) are widely prescribed nowadays since their introduction in the late 1980s. They have exhibited a good tolerability and safety profile. Nevertheless, some ADRs can arise during SSRI treatment, which may lead to poor treatment adherence or ultimately treatment discontinuation [3]. It is unknown whether gender differences exist in the occurrence of ADRs when using SSRIs.

Aim: The aim of the present study was to investigate whether differences exist in the number of reported ADRs of SSRIs for men and women in the database of the Netherlands Pharmacovigilance Centre Lareb.

Methods: All SSRI reports entered into the database from 1 January 2003 until 31 December 2015 were included in this study.

Disproportionality based on reporting odds ratios (RORs) was calculated for all the preferred terms (PTs) reported on SSRIs for men and women. These RORs were corrected for the number of females and males using SSRIs in the Netherlands using the GIP database. This is an information system of National Health Care Institute containing information on expenditure on drugs in the Netherlands and the degree to which they are used [4]. A statistically significant ROR $>1$ indicates that a certain PT is reported more in females than males, whereas a statistically significant ROR $<1$ indicates that a certain PT is reported more in males than females. SSRI-PT associations that were mentioned in at least 50 reports were assessed. Gender specific ADRs, like amenorrhoea, metrorrhagia and erectile dysfunction were excluded from the analysis.

Results: 15 PTs were statistically significantly more reported in females than in males. 3 PTs were statistically significantly more reported in males than females (Table I). Woman were more likely to report dose-related adverse effects

Conclusions: This explorative study on the influence of gender on reported ADRs when using SSRIs shows that there are several ADRs (especially doserelated adverse effects) that are reported more in females than males.

Further sources of information/References

1. Franconi F, Brunelleschi S, Steardo L, Cuomo V. Gender differences in drug responses. Pharmacol Res 2007; 55: 81-95

2. Zopf Y, Rabe C, Neubert A, Gassmann KG, Rascher W, Hahn EG, et al. Women encounter ADRs more often than do men. Eur J Clin Pharmacol 2008; 64: 999-1004

3. Kostev K, Rex J, Eith T, Heilmaier C. Which adverse effects influence the dropout rate in selective serotonin reuptake inhibitor (SSRI) treatment? Results for 50,824 patients. Ger Med Sci 2014; 12: Doc15

4. GIPdatabase-Drug Information System of the Dutch Health Care Insurance Board. http://www.gipdatabank.nl

Table I. Number of reports in females and males and ROR
\begin{tabular}{|l|l|l|l|}
\hline Preferred term & Number of reports (F) & Number of reports (M) & ROR (95\%CI) \\
\hline Haematoma & 93 & 7 & $6.8(3.2-14.7)$ \\
\hline Alopecia & 102 & 16 & $3.3(1.9-5.6)$ \\
Purpura & 44 & 7 & $3.2(1.5-7.2)$ \\
\hline Weight increased & 198 & 40 & $2.5(1.8-3.6)$ \\
Arthralgia & 50 & 11 & $2.3(1.2-4.5)$ \\
Vision blurred & 62 & 14 & $2.3(1.3-4.1)$ \\
Palpitations & 90 & 22 & $2.1(1.3-3.4)$ \\
Dry mouth & 85 & 21 & $2.1(1.3-3.4)$ \\
Dizziness & 228 & 60 & $2.0(1.5-2.6)$ \\
\hline Hyponatraemia & 93 & 25 & $1.9(1.2-3.0)$ \\
Abdominal pain & 62 & 17 & $1.9(1.1-3.2)$ \\
Tremor & 147 & 45 & $1.7(1.2-2.3)$ \\
Hyperhidrosis & 214 & 74 & $1.5(1.1-1.9)$ \\
Nausea & 267 & 94 & $1.5(1.2-1.9)$ \\
Fatigue & 148 & 54 & $1.4(1.0-1.9)$ \\
\hline Aggression & 63 & 70 & $0.5(0.3-0.7)$ \\
Suicide attempt & 35 & 38 & $0.5(0.3-0.8)$ \\
Tinnitus & 61 & 63 & $0.5(0.4-0.7)$ \\
\hline
\end{tabular}

\section{Multi-Level Modelling (MLM) in Specialist Cohort Event Monitoring (SCEM) Studies}

\author{
D. Layton ${ }^{1,2}$, D. Roy ${ }^{1,2}$, S. Marley ${ }^{3}, \underline{\text { S. Shakir }}{ }^{1,2}$ \\ ${ }^{1}$ Drug Safety Research Unit, Southampton, UK, ${ }^{2}$ University \\ of Portsmouth, Portsmouth, UK, ${ }^{3}$ Select Statistical Services Ltd, \\ Exeter, $U K$
}

Background: Prescribing guidelines influence treatment choice based on patient factors. Clinical use is also influenced by non-patient factors. MLM can provide insight into sources of variability in healthcare, where hierarchical structures exist [1]. A SCEM study is investigating the safety and use of rivaroxaban in clinical use, with a warfarin cohort for context. Objective: Ad-hoc MLM to explore influences on prescribing anticoagulants.

Methods: Data on 53 NHS acute trusts in England and Wales (e.g. population size, prescribing guidelines) were linked to interim SCEM patient demographic and drug utilization data, and prescriber details (e.g. degree, specialism). MLM was applied to 1006 (55\%) rivaroxaban vs. 816 warfarin $(45 \%)$ adult patients nested in 514 prescribers, nested in trusts. The binary outcome was rivaroxaban or warfarin treatment. Variance components were expressed as median odds ratios (MOR-median relative increase in odds of rivaroxaban treatment if patient changed prescriber/ trust) and proportional change in variance (PCV) between models when successively adding fixed effects (patient/prescriber/trust). If treatment choice was dominated by patient factors, having accounted for their effects, variance between prescribers and trusts would be comparatively low.

Results: Differences between trusts and prescribers in trusts are important in treatment choice; trust being more influential (MOR ${ }_{\text {TRUST }}$ (T) $=6.9$; MOR PRESCRIBER (P) $=3.9$ ). Some patient factors had a relatively large effect on odds of treatment choice, e.g. cerebrovascular accident [OR 2.0 $\left(95 \%\right.$ CI 1.3, 3.0)]. Adjusting for patient factors, $\mathrm{MOR}_{\mathrm{T}}=7.8$ $(\mathrm{PCV}=20 \%) ; \mathrm{MOR}_{\mathrm{P}}=4.5(\mathrm{PCV}=14 \%)$. Adjusting for prescriber factors, $\mathrm{MOR}_{\mathrm{T}}=7.1 \quad(\mathrm{PCV}=-8 \%) ; \mathrm{MOR}_{\mathrm{P}}=3.8 \quad(\mathrm{PCV}=-19 \%)$. Adjusting for trust factors did not improve the model performance.

Conclusions: In this exploratory analysis, treatment variability appears dominated by differences between trusts and prescribers; most notably trusts. Some patient factors were important in treatment choice, but PCV between models suggest that accounting for patient differences does not fully explain the variance between prescribers and between trusts. This study highlights the utility of MLM in exploring non-patient factors. This interim analysis will be superseded on completion of the SCEM study.

\section{Further sources of information/References:}

1. Zuidgeest MG, van Dijk L, Spreeuwenberg P, Smit HA, Brunekreef $\mathrm{B}$, Arets HG, et al. What drives prescribing of asthma medication to children? A multilevel population-based study. Ann Fam Med. 2009;7:32-40

\section{Are NSAIDs really cheap medications?}

M. Ponte ${ }^{1}$, M. Froján ${ }^{1}$, J.M. Fernandez Acuña ${ }^{1}$, L.N. Noferi ${ }^{1}$, J.F. Vignale ${ }^{1}$, L.R. Zielinski ${ }^{1}$, F. Paso ${ }^{1}$, H.A. Serra ${ }^{1}$

${ }^{1}$ Pontificia Universidad Catolica Buenos Aires, Ciudad Autonoma De Buenos Aires, Argentina 
Background: There is a great concern nowadays in medicine about new drug̈̈ or"monoclonal antibodies̈ or"generic̈̈ or"biosimilars̈ and their clinical and economic burden. But slowly we have forgotten another great problem of the true costs of cheap medications to the medical system. Aim: the aim of this study is to show that normally thought as "cheap medications could be as expensive to the medical system an" new drugs̈. Methods. We evaluated a pharmacovigilance database of a tertiary care hospital. We separated two great NSAIDs-induced ADR (gastrointestinal bleeding and renal failure) and we performed the economic (direct and indirect) burden of this ADR.

Results: For a population of approximately 200,000 inhabitants, the direct and indirect economic burden of NSAIDs-induced ADR is 347,749.73 US dollars per year. This is near one fourth of the economic burden of hemophilic treatment according to prevalence in this population or one third of the economic burden of Gaucher disease according to prevalence in this population.

Discussion: Despite NSAIDs could be cheap medications for the patient, they generate extremely high economic burden for the health system. We urgent need to encourage professional community and general population to use cautiously this medication.

\section{Characteristics of adverse events following influenza vaccination: comparison between spontaneous reports and reports from an intensive monitoring programme}

L. van Balveren-Slingerland ${ }^{1}$, S. Vorstenbosch ${ }^{1}$, E. van Puijenbroek

\section{${ }^{1}$ Lareb, 's-Hertogenbosch, the Netherlands}

Introduction: Most reported adverse events (AEs) following influenza vaccination are well-known. One commonly used method to retrieve information on AEs is spontaneous reporting. Information concerning the course of reported AEs is highly dependent on the time of reporting. If reported in an early stage, this information cannot yet be provided. Data can also be obtained through intensive monitoring of patients, thereby facilitating to report (the course) of AEs. Both methods provide information concerning the frequencies of reported AEs, latency time, outcome and recovery time. However, whether or not both approaches yield comparable information in respect to the aforementioned aspects, is unknown.

Aim: To determine differences between spontaneous reporting and an intensive monitoring programme in frequencies of reported AEs, latency time and recovery status and -time of the reported AEs.

Methods: Comparison between the characteristics of most reported AEs reported in spontaneous reports and in an intensive monitoring programme following influenza vaccination. All spontaneous reported AEs of patients received within 40 days after influenza vaccination in 2013, 2014 and 2015 were included. In the same years an intensive monitoring programme was performed. Patients received three questionnaires on day 5, 15 and 30 (valid thru day 40) after vaccination. Of the ten most reported AEs the recovery status, latency- and recovery time were calculated. The latencyand recovery time were categorized into four groups for different intervals $(0-24 ; 24-48 ; 48-72 ;>72 \mathrm{~h})$. To compare the early $(<72 \mathrm{~h})$ and late $(>72$ h) latency- and recovery times between both methods, Fisher Exact Tests were performed.

\begin{tabular}{|l|l|l|}
\hline & $\begin{array}{l}\text { \% of total reported } \\
\text { AE: spontaneous } \\
(1488 \mathrm{AE}) \text { vs } \\
\text { monitor }(2588 \mathrm{AE})\end{array}$ & $\begin{array}{l}\text { \% recovered AE: } \\
\text { spontaneous vs } \\
\text { monitor }\end{array}$ \\
\hline Reported AE & $21.3-22.4$ & $25.0-76.7$ \\
\hline Injection site inflammation & $9.7-14.9$ & $36.6-77.7$ \\
\hline Myalgia & $11.1-14.3$ & $43.2-79.5$ \\
\hline Headache & $8.2-5.6$ & $46.7-80.0$ \\
\hline Pyrexia & $2.1-5.6$ & $45.2-84.0$ \\
\hline Injection site pain & $4.0-1.8$ & $29.3-72.3$ \\
\hline Extensive swelling of vaccinated limb & $2.1-2.4$ & $32.3-54.7$ \\
\hline Fatigue & $2.8-3.0$ & $29.3-72.7$ \\
\hline Malaise & $2.7-2.8$ & $48.7-68.1$ \\
\hline Influenza like illness & &
\end{tabular}

Results: The most reported AEs in both methods were comparable. The table below shows the frequencies of occurrence of these reported AEs. In both methods most reported AEs started within $72 \mathrm{~h}$ after vaccination. Significant differences were seen for the latency time of pyrexia, headache and malaise. These AEs were more frequently reported after $72 \mathrm{~h}$ in the monitoring programme. Data on recovery was more frequently available in the intensive monitoring (see Table). Yet, no differences were seen between the recovery time of reported events for both methods.

Conclusion: This study shows that the pattern of reported AEs is comparable between both methods. Possibly more events in the monitoring programme were reported because patients were monitored over time and asked to report the (course of) AEs. Consequently, more data on recovery was available, which illustrates the strength of monitoring in providing indepth information about the course of the AEs.

\section{Deaths from medicines: a systematic analysis of UK Coroners' reports to prevent future deaths}

\author{
A. R. Cox ${ }^{1,2}$, C. Easton ${ }^{1}$, R.E. Ferner ${ }^{1,2}$ \\ ${ }^{1}$ Institute of Clinical Sciences, University of Birmingham, \\ Birmingham, UK, ${ }^{2}$ West Midlands Centre for Adverse Drug \\ Reactions, Birmingham, UK
}

Introduction: Coroners in England and Wales investigate unexplained deaths. Since legislation in 2009 , they must make reports following deaths where they believe it is possible to prevent future deaths (PFDs). [1] PFDs are sent to relevant people or organizations, asking for recommendations on how to prevent future deaths. They could provide information relevant to pharmacovigilance.

Aim: We wished to establish how often PFDs implicated medicines and other drugs, the extent to which they revealed preventable medication errors or novel adverse drug reactions, and the concerns raised by coroners.

Methods: We examined coroners' reports by pre-defined criteria to identify those in which medicines played a part, and to collect information on coroners' concerns. We included a PFD if it mentioned part of the medication process (for example, administration), or if a specific medicine (or both) that caused or contributed to death. We included drugs of abuse such as diamorphine (heroin) and cocaine. We excluded cases where the medicine or part of the medication process mentioned did not cause or 
contribute to death; and where delays in assessment, investigation, or diagnosis led to delays in treatment. We also excluded cases concerning only alcohol.

Results: We identified 99 reports (100 deaths) in which medicines or a part of the medication process or both contributed to death. Reports mentioned anticoagulants (25 deaths), opioids (24), antidepressants (18), drugs of abuse excluding opioids (13), and other drugs. The most important concerns related to adverse reactions to prescribed medicines (22), omission of necessary treatment (21), failure to monitor treatment (17), and poor systems (17). (Table 1) Almost all were well known. Coroners related them principally to defects in education or training, lack of clear guidelines or protocols, and failure to implement existing guidelines. Most reports went to healthcare organisations. Responses to PFDs were rarely published. We identified four safety warnings from the UK's Medicines and Healthcare products Regulatory Agency that were based on coroners' warnings. [2-5]

Conclusions: Coroners' reports to Prevent Future Deaths provide some information on medication errors and adverse reactions relevant to pharmacovigilance. The majority report previously well-known problems. Many reports of deaths associated with medicines are sent to local organizations, and wider regulatory lessons may be lost.

\section{Further sources of information/References}

1. https://www.judiciary.gov.uk/related-offices-and-bodies/office-chiefcoroner/pfd-reports/

2. Anonymous. Citalopram: suspected drug interaction with cocaine; prescribers should consider enquiring about illicit drug use. Drug Saf Update 2016; 9 (12): 2

3. Anonymous. Hyoscine butylbromide (Buscopan) injection: risk of serious adverse effects in patients with underlying cardiac disease. Drug Saf Update 2017; 10 (7): 1

4. Anonymous. Spironolactone and renin-angiotensin system drugs in heart failure: risk of potentially fatal hyperkalaemia. Drug Saf Update 2016; 9 (7):2

5. Anonymous. Topical miconazole, including oral gel: reminder of potential for serious interactions with warfarin. Drug Saf Update 2016; 9 (11): 3

Table 1: Top seven concerns raised in UK Coroners' reports concerning medicines.

\begin{tabular}{|l|l|}
\hline Concern & $\begin{array}{l}\text { Number of } \\
\text { occurrences }\end{array}$ \\
\hline ADR to prescribed medicines & 22 \\
\hline Omission of necessary treatment & 21 \\
\hline Monitoring failure & 17 \\
\hline Poor systems & 17 \\
\hline Poor communication & 13 \\
\hline Drug regulation inadequate [or failure to enforce] & 9 \\
\hline Interaction & 7 \\
\hline
\end{tabular}

\section{Supporting Pharmacovigilance by Landscaping EU-funded Real World Data}

\author{
K. Plueschke ${ }^{1}$, X. Kurz ${ }^{1}$, A. Cave ${ }^{1}$, P. McGettigan ${ }^{1}$, \\ A. Pacurariu $^{1}$ \\ ${ }^{1}$ European Medicines Agency, Canary Wharf, London, UK
}

Background: There is increasing interest in the use of real world data (RWD) to support pharmacovigilance activities. Substantial European funding has addressed the numerous challenges associated with these datasources, including data heterogeneity, access, and governance. Multiple parallel initiatives may however increase the risk of duplication and the potential for generating unsustainable data sources.

Aim: To create an inventory of EU-funded healthcare-related initiatives linked to RWD to provide a systematic mean of identifying EU datasources and expertise to support regulatory pharmacovigilance activities. Methods: Key EU-funded programmes were interrogated to identify initiatives linked to RWD. Using publicly available information, initiatives were characterised according to their primary goal: (1) creation of new 'real-world' data-sources, (2) development of new methodologies for RWD, (3) governance models for 'real world' studies, (4) analytical models to collect/link/transform RWD, or (5) infrastructure to store and share RWD. A dashboard was created to score the initiatives' outputs and enable identification of key attributes. Lastly, owners of selected datasources were contacted to understand potential mechanisms for regulatory access.

Results: Of 171 initiatives screened, 68 were relevant for the inventory. On preliminary classification, 35 were novel 'real world' data-sources, 6 were methodologies, 3 were governance models, 12 were analytical models and 12 were infrastructure. The mean funding period was 5 years and ranged from 1 million euros to 56 million euros for a single initiative, and partners ranged from 2 to above 50. The number of patients included were highly variable (median: 10,000, range: 350-40 million). 'Real world' data-sources initiatives clustered into specific disease areas, e.g. $37 \%$ covered Central Nervous System- or Respiratory diseases-focused (Table 1). Outputs matched the stated objectives for 13 of the 23 finalised initiatives. Limitations identified included restricted or no access to data for non-participants and lack of funding sustainability beyond the funding period.

Conclusion: The creation of the inventory delivered an understanding of the RWD landscape in Europe and expanded regulatory knowledge of RWD expertise potentially capable of contributing to a multi-national network of pharmacovigilance activities. Acknowledging the limitations of RWD, the outputs could contribute to evidence-based decision-making, in line with the EU Network Strategy to 2020.

Table 1: Number of initiatives per specific disease areas

\begin{tabular}{|l|l|}
\hline Specific disease areas & Number of initiatives (\%) \\
\hline Central Nervous System & $7(20)$ \\
\hline Respiratory & $6(17)$ \\
\hline Rheumatology & $3(8.6)$ \\
\hline Oncology & $5(14.3)$ \\
\hline Immunology & $3(8.6)$ \\
\hline Metabolism & $2(5.7)$ \\
\hline Pregnancy & $1(2.8)$ \\
\hline Rare diseases & $1(2.8)$ \\
\hline Any & $7(20)$ \\
\hline
\end{tabular}

32 Concurrent Use of Prescription Medicines and Herbal Medicinal Products among Older Adults: A Systematic Review

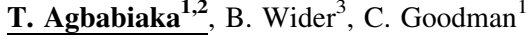 \\ ${ }^{1}$ Centre for Research in Primary \& Community Care, University \\ of Hertfordshire, Hatfield, UK, ${ }^{2}$ Patient Safety Team. NHS
}


Improvement, London, UK, ${ }^{3}$ Institute of Health Research. University of Exeter Medical School, Exeter, UK

Background: Polypharmacy or multiple use of medicines is very common among older adults. [1, 2] A significant number of older adults concurrently use prescription medicines with herbal medicinal products (HMPs). $[3,4]$ Due to interactions between conventional drugs and HMPs, there is potential for adverse drug reaction (ADR) to occur, some with serious consequences [5, 6]. Older people are at greater risks of ADR due to comorbidities and reduced clearance of pharmacologically active compounds with increasing age. [7] Little is known of the extent and implications of older adults' concurrent use of prescription medicines with HMPs.

Aims: To assess and synthesise available evidence on the prevalence, patterns of use and potential herb-drug interactions associated with the concurrent use of prescription medicines and HMPs among older adults. Methods: Systematic searches of Medline, PsychInfo, Embase, CINAHL, AMED, Web of Science and Cochrane until April 2016, for studies reporting concurrent use of HMPs with prescription medicines among adults aged $\geq 65$ years. And lateral searches via related citation in PubMed and checking reference lists of identified studies. Studies reporting use of other complementary and alternative remedies, animal studies and nonempirical papers were excluded. Two reviewers independently screened studies, extracted data and appraised methodological quality using the Joanna Briggs Institute (JBI) checklist Institute checklist for prevalence data [8]. A narrative synthesis of included studies was done to summarise the evidence.

Results: The literature screening identified 2176 records from which 20 were included in the analysis. The majority of studies were conducted in the United States. The definition of HMPs varied and some papers also assessed non-herbal dietary supplements, vitamins and minerals. Only 14 studies reported the prevalence of concurrent use between prescriptions and HMPs. Prevalence varied widely between 5 and $83 \%$, mean prevalence was $32.9 \%$. The most commonly combined HMPs were gingko, garlic, ginseng, St John's wort, Echinacea, Saw palmetto, evening primrose and ginger. The most reported prescription medicines were antihypertensives, diuretics, antihyperlipidemic agents, anticoagulants, analgesics, antihistamines, antidiabetics and antidepressants. A total of 2697 potential interactions were reported in 14 studies. The majority of interactions involved risk of bleeding due to use of ginkgo, garlic, ginseng together with aspirin or warfarin.

Conclusion: Despite the heterogeneity in definition of herbal medicines and varying prevalence from included studies, this review established that concurrent use is substantial among older adults, identified range of HMPs, prescription medicines frequently combined and the potential interactions from such combinations.

Further sources of information/References

1. Chen YF, Dewey M, Avery A. Self-reported medication use for older people in England and Wales. J Clin Pharm Ther 2001; 26:129-140

2. Leiss W, Méan M, Limacher A, Righini M, Jaeger K, Beer H-J, et al. Polypharmacy is associated with an increased risk of bleeding in elderly patients with venous thromboembolism. J Gen Intern Med 2015; 30:17-24

3. Ly J, Percy L, Dhanani S. Use of dietary supplements and their interactions with prescription drugs in the elderly. Am J Health Syst Pharm 2002; 59:1759-1763

4. Qato DM, Alexander GC, Conti RM, Johnson M, Schumm P, Lindau ST. Use of prescription and over-the-counter medications and dietary supplements among older adults in the United States. JAMA 2008; 300

5. Fugh-Berman A. Herb-drug interactions. Lancet 2000; 355:134-138
6. Williamson EM, Driver S, Baxter K. Stockley's Herbal Medicines Interactions. In. Second ed. London: Pharmaceutical Press; 2013. pp. 512

7. Davies EC, Green CF, Taylor S, Williamson PR, Mottram DR, Pirmohamed M. Adverse drug reactions in hospital in-patients: a prospective analysis of 3695 patient-episodes. PLoS One 2009; 4(2):e4439

8. The Joanna Briggs Institute. Reviewers manual. South Australia: The University of Adelaide; 2014

\section{Pharmacovigilance and Rational Drug Use Content in Pharmacology Education in Medical Schools in Turkey}

\section{M.D. Güner' ${ }^{1}$, P.E. Ekmekci ${ }^{2}$}

${ }^{1}$ Tobb Economy and Technology University Faculty of Medicine Department of Medical Pharmacology, Ankara, Turkey, ${ }^{2}$ TOBB Economy and Technology University, Faculty of Medicine Department of History of Medicine and Ethics, Ankara, Turkey

Background: Adverse drug reaction (ADR)-reporting rate of Turkey is 2-reports/million-inhabitants/year and it is below the average of similarincome countries [1]. Scarceness of pharmacovigilance knowledge may be the factor limiting reporting rate. The level of content of pharmacovigilance in medical schools is the essential determinant of this knowledge. The future doctors' knowledge on pharmacovigilance and ADR-reporting is below the desired and required levels [2].

Objective/Aim: To evaluate the content of pharmacovigilance and related subjects in the syllabi of the medical schools.

Methods: The syllabi of the 81 medical schools in Turkey as provided on their web pages were evaluated for the following subjects: pharmacovigilance, rational drug use, drug use in geriatric/paediatric populations and during pregnancy, drug toxicity, and ADR-reporting. The number of hours dedicated and at what phase the course is provided were also evaluated.

Results: Of 81 medical schools, 38 provide at least $1 \mathrm{~h}$ of pharmacovigilance course. Similarly, drug toxicology (37), prescription writing (34), rational drug use (33), rational antibiotic use (10), and drug use in pregnancy and lactation (22), geriatric population (16), and paediatric population (11) were presented at least for $1 \mathrm{~h}$ and mostly at 3rd year of medical school. Pharmacoeconomics was provided by two, and ADR reporting, legal aspects of medication/drug prescription, or counterfeit drugs by one medical school.

Only 16 of the medical schools had clinical pharmacology stage at 4th or 5 th year of the medical school. The clinical pharmacology stage provide courses on rational drug use, personal-drug selection, and clinical pharmacology of organ-systems.

Conclusion: In order to increase the number of ADR-reporting, more time and effort must be allocated to pharmacovigilance education in all phases of medical school.

Discussion: Most pharmacology education in medical schools occurs in the early years. Evaluation of the syllabi showed that the time allocated for pharmacovigilance and related subjects is not sufficient to acquire a knowledge that will make a change in the future doctors' attitude and behaviours. Providing the subject during the later phases may make a difference [3], however, the course duration must be increased and ADR reporting practice must be included.

We suggest that at least 1-week of pharmacovigilance compulsory course should be provided covering the basic elements of pharmacovigilance 
education and training $[4,5]$, in order to increase the ADR reporting rates during the following decades.

Further sources of information/References:

1. Aagaard L, Strandell J, Melskens L, Petersen PS, Holme Hansen E. Global patterns of adverse drug reactions over a decade: analyses of spontaneous reports to VigiBase ${ }^{\mathrm{TM}}$. Drug Saf. 2012; 35:1171-82.

2. Schutte T, Tichelaar J, Reumerman MO, van Eekeren R, Rissmann R, Kramers C, et al. Pharmacovigilance Skills, Knowledge and Attitudes in our Future Doctors-A Nationwide Study in the Netherlands. Basic Clin Pharmacol Toxicol. 2017; 120:475-481

3. Arici MA, Gelal A, Demiral Y, Tuncok Y. Short and long-term impact of pharmacovigilance training on the pharmacovigilance knowledge of medical students. Indian J Pharmacol. 2015; 47:436-9

4. Beckmann J, Hagemann U, Bahri P, Bate A, Boyd IW, Dal Pan GJ, et al. Teaching pharmacovigilance: the WHO-ISoP core elements of a comprehensive modular curriculum. Drug Saf. 2014; 37:743-59

5. Guideline on good pharmacovigilance practices http://www.ema. europa.eu/ema/index.jsp?curl=pages/regulation/document_listing/ document_listing_000345.jsp Last visited 22 April 2017

\section{Turkish physicians' pharmacovigilance and adverse drug reaction reporting knowledge, attitude, and practices}

\section{M.D. Güner ${ }^{1}$, P.E. Ekmekci ${ }^{2}$, S.E. Öziş ${ }^{3}$}

${ }^{I}$ Tobb Economy and Technology University Faculty of Medicine Department of Medical Pharmacology, Ankara, Turkey, ${ }^{2}$ Tobb

Economy \& Technology University Faculty of Medicine Department of History of Medicine and Ethics, Ankara, Turkey, ${ }^{3}$ Tobb Economy \& Technology University Faculty of Medicine Department of General Surgery, Ankara, Turkey

Background: Adverse drug reaction (ADR)-reporting rate of Turkey is 2-reports/million-inhabitants/year and it is below the average of similarincome countries [1]. The limited number of studies evaluating this indicate the scarceness of pharmacovigilance knowledge; which may be the factor limiting the reporting rate. As pharmacovigilance education is limited at undergraduate level, physicians may not sufficiently have the knowledge of pharmacovigilance practices.

Objective/Aim: To evaluate the knowledge, attitude and practices (KAP) of pharmacovigilance activities of medical doctors.

Methods: We searched the literature for the questionarres evaluating KAP of physicians. We gathered $\sim 70$ questions to evaluate the following subjects:

A. Sociodemographic characteristics (10-questions)

1. Qualified person for pharmacovigilance: if a physician has/had this responsibility 8-questions related to the activities and responsibilities are asked.

2. Pharmacovigilance and ADR reporting KAP (40-questions)

3. Drug use, prescription habits and communication of ADRs with patients (11-questions)

The online survey is presented to a small group of physicians in order to understand the feasibility and applicability. We will contact Turkish Medical Association (TMA), share the details of the study, and request help for dissemination of survey. TMA has $\sim 80,000$ members and use electronic communication systems effectively. The survey will be disseminated via their system.
The survey webpage will open with information about the study and after only accepting to voluntarily answer the questions by check marking, the volunteer will be directed to questions page. The number of questions will change between 58 and 69 depending on their answers.

Results: Literature search showed that the ADR reporting number of Turkey is very small (2455 reports in 2013 and most of these were reported by physicians $(59.8 \%)$ [1,2]. The main reason for underreporting is limited knowledge of pharmacovigilance and ADR reporting system. Based on the data collected from our focus group, physicians don't know the essentials of pharmacovigilance, ADR reporting system in Turkey, and their role in this system.

Conclusion: Urgent incentives are needed to increase the awareness of physicians on pharmacovigilance system in Turkey as their scarce knowledge is the main factor limiting the number of ADR reports.

Discussion: It is estimated that at least 5\% of the TMA members will join the survey which will provide data on pharmacovigilance KAP of $\sim 4000$ medical doctors. This will be a comprehensive study evaluating the subject and will provide information to academicians and authorities to direct the incentives to increase the pharmacovigilance knowledge and then increase the number of ADR reports.

Further sources of information/References:

1. Aagaard L, Strandell J, Melskens L, Petersen PS, Holme Hansen E. Global patterns of adverse drug reactions over a decade: analyses of spontaneous reports to VigiBase ${ }^{\mathrm{TM}}$. Drug Saf. 2012; 35:1171-82

2. Ozcan G, Aykac E, Kasap Y, Nemutlu NT, Sen E, Aydinkarahaliloglu ND. Adverse Drug Reaction Reporting Pattern in Turkey: Analysis of the National Database in the Context of the First Pharmacovigilance Legislation. Drugs Real World Outcomes. 2016; 3:33-43

\section{Towards Web-Based, Purchase-Event Intensive Monitoring for Pharmacovigilance of Natural Health Products (NHPs): Lessons From Development Work}

\author{
J. Barnes $^{1}$, J. Sheridan ${ }^{1}$, M. Harrison-Woolrych ${ }^{2}$, C. Dong ${ }^{1}$, \\ L. Härmark $^{3}$, S. Vohra ${ }^{4}$ \\ ${ }^{I}$ School of Pharmacy, University Of Auckland, Auckland, New \\ Zealand, ${ }^{2}$ Dunedin School of Medicine, University of Otago, \\ Dunedin, New Zealand, ${ }^{3}$ Netherlands Pharmacovigilance Centre \\ Lareb, 's-Hertogenbosch, the Netherlands, ${ }^{4}$ Dept of Pediatrics \\ and Medicine, Faculty of Medicine \& Dentistry and School of Public \\ Health, University of Alberta, Edmonton, Canada
}

Background: Identification of adverse drug reactions (ADRs) associated with natural health products (NHPs) relies on voluntary spontaneous reporting to national schemes, but relatively few NHP-ADR reports are submitted. [1-3] There is international recognition of the need to improve pharmacovigilance for NHPs, including through developing methods building on existing systems, such as intensive monitoring. [4,5]

Aim: To examine feasibility issues for purchase-event intensive monitoring for NHPs.

Methods: Two studies were conducted. Study 1: single-/multi-ingredient NHPs containing the herbal ingredient Ginkgo biloba ('ginkgo') leaf were selected for monitoring; study 2 monitored all NHPs sold in participating pharmacies. Community pharmacies were recruited through postal invitations sent to all Auckland pharmacies (August 2015; study 1), and all Green Cross Health (a nationwide primary-healthcare network) pharmacies (April 2016; study 2). Participating pharmacies received an audit visit and study training via Skype (study 1); in study 2 , 
pharmacists were emailed a training presentation and audited by telephone. During consumer recruitment (study 1:3 months; study 2: 2 weeks), pharmacy staff gave uniquely numbered study invitation cards to all customers purchasing ginkgo/NHPs (study 1/2). Customers contacting the study team and providing informed consent were enrolled. Consumer participants were emailed links to web-based baseline and follow-up questionnaires (recording use of NHP purchased, medical history, concurrent medicines, adverse events (AEs)) using Qualtrics software. Feedback data on study methods were collected from pharmacist and consumer participants in both studies through interviews/web-based questionnaires. The University of Auckland Human Participants Ethics Committee approved the studies.

Results: Three pharmacies (1.4\%) participated in study 1; no consumers were recruited. In study 2,18 pharmacies $(5.4 \%)$ participated and 4 consumers were enrolled ( $<0.5 \%$ of those given a study invitation card). All consumer participants reported taking purchased NHP(s) with other medicines (range 3-9) and described one or more current chronic health conditions. Three consumers completed the follow-up questionnaire; one reported an AE, categorised 'serious' (caused hospitalisation). In study 2, $>90 \%$ of pharmacist respondents agreed they participated because they had a strong personal interest in NHPs and safety of NHPs, were concerned about safety of NHPs, and because NHPs were an important sales category in their pharmacy.

Conclusion: Low recruitment of pharmacists/pharmacies and NHP-purchasers renders these studies unfeasible at present. Monitoring safety of NHPs, however, remains important. While challenging, reasons for low participation need to be examined towards finding solutions to increasing reporting rates for NHP ADRs. This is particularly important given the contribution patient reporting makes to detecting signals of medicines' safety concerns.

Further sources of information/References:

1. Farah $\mathrm{MH}$, Edwards R, Lindquist M, Leon $\mathrm{C}$, Shaw D. International monitoring of adverse health effects associated with herbal medicines. Pharmacoepidemiol Drug Saf 2000;9:105-12

2. Barnes J, Tatley M. Scant reporting on CAMs safety in NZ. Pharmacy Today 2007;Feb:6

3. Barnes J, Mills SY, Abbot NC, Willoughby M, Ernst E. Different standards for reporting ADRs to herbal remedies and conventional OTC medicines: face-to-face interviews with 515 users of herbal remedies. Br J Clin Pharmacol 1998;45:496-500

4. Barnes J. Pharmacovigilance of herbal medicines. A UK perspective. Drug Safety 2003;26:829-851

5. World Health Organisation. WHO guidelines on safety monitoring of herbal medicines in pharmacovigilance systems. Geneva: WHO, 2004

\section{Drug-induced bone loss: A study of the French and Spanish PharmacoVigilance DataBases}

\author{
Q. Dardonville ${ }^{1}$, E. Salguiero ${ }^{2}$, V. Rousseau ${ }^{1}$, D. Abadie ${ }^{1}$, \\ G. Manso $^{2}$, J.L. Montastruc ${ }^{1}$, H. Bagheri ${ }^{1}$
}

${ }^{1}$ Service De Pharmacologie Médicale Et Clinique, Centre De Pharmacovigilance, Toulouse, France, ${ }^{2}$ Department of Medicine, Pharmacology, Faculty of Medicine, University of Oviedo, Asturias, Spain

Introduction: Osteoporosis, the most prevalent bone disorder, is a skeletal disease characterized by a systemic impairment of bone mass, strength and microarchitecture which increases the propensity of fragility fractures.
Corticoids are well known to induce bone loss. However, involvement of other drugs remains discussed. The aim of this study was to analyze the characteristics of drug-induced osteoporosis in the French and Spanish PharmacoVigilance DataBases (PVDB).

Material and methods: All cases of drug-induced osteoporosis registered in French and Spanish PVDB were extracted until 31 December 2015. All adverse drug reactions (ADRs) registered as « osteopenia » or « osteoporosis» according to the Medical Dictionary for Drug Regulatory Activities were selected. Descriptive analysis was performed for demographic data, ADRs and their seriousness, risk factors and suspected drugs. The comments of all reports were reviewed in order to exclude all cases which not corresponded to osteoporosis. Furthermore, a case/no case study was performed in the French PVDB for two classes: Proton Pomp Inhibitors (PPI) and serotoninergic recapture inhibitors (SRI).

Results: French analysis included 318 patients with a mean age of $57.8 \pm 18.7$ years and $55.7 \%$ of men. In 147 cases (46.2\%), ADRs led to bone lesions like fractures. ADRs were serious in $41.5 \%$ of cases $(\mathrm{n}=132)$. The mean number of suspected drugs was $2.3 \pm 1.8$.

Spanish analysis included 42 patients with a mean age of $49.9 \pm 20.3$ years and $40.0 \%$ of men. In 15 patients (35.7\%), ADRs led to bone lesions. ADRs were serious for $81 \%$ of cases $(n=34)$. The mean number of suspected drugs was $2.0 \pm 1.5$.

In order to remove the risk of corticoids exposure (corresponding to glucocorticoids dose $>7.5 \mathrm{mg}$ prednisone equivalency more than 3 months) bias, a total of 81 cases were excluded. Then, the most suspected drugsinduced osteoporosis were: antivirals (92 cases), corticosteroids short-term exposure ( 90 cases) and anti-epileptics (42 cases).

The case/no case analysis study did not show any significant association for SRI (OR $=0.45[0.18-1.07])$. However, the association was significant for PPI exposure (OR $=1.81[1.01-3.22]$ ).

Conclusion: Data of French and Spanish PVDB show that osteoporosis is not only restricted to GCs. Other drugs like mainly antivirals, antiepileptics or PPI are involved. Their effect can be explained by their pharmacological properties. Further studies should be undertaken in order to assess SRI-induced osteoporosis as suggested in the literature.

\section{Dolutegravir Withdrawal and Adverse Drug Reactions: A Retrospective Study in Midi-Pyrénées}

\author{
O. Phouthavong ${ }^{1}$, L. Cuzin ${ }^{2}$, A. Sommet ${ }^{1}$, J.L. Montastruc ${ }^{1}$, \\ H. Bagheri ${ }^{1}$ \\ ${ }^{I}$ Service De Pharmacologie Médicale Et Clinique, Centre De \\ Pharmacovigilance, Toulouse, France, ${ }^{2}$ INSERM, UMR 1027,; \\ Université de Toulouse III, Toulouse, France
}

Objective: Dolutegravir (DTG) is an integrase inhibitor blocking an enzyme called integrase involved in the integration of viral DNA in the host lymphocyte cells. It was marketed in Europe from 2014 as Tivicay ${ }^{\circledR}$ or Triumeq ${ }^{\circledR}$ (lamivudine + DTG + abacavir) in association to other drugs for Viral Human Immunodeficiency (HIV). Our study was undertaken in order to describe the characteristics of patients in whom DTG was withdrawn due to Adverse Drug Reactions (ADRs) in Midi-Pyrénées.

Method: We performed a retrospective study (2014-2016) in patients seeking care in Toulouse University Hospital, exposed to DTG. Data were extracted from Nadis ${ }^{\circledR}$ software. Nadis ${ }^{\circledR}$ (Fedialis Medica) is a medical electronic record for HIV, HBV and HCV patients consulting in French public hospitals. Patients exposed to DTG during this period were selected. Furthermore, characteristics of patients in whom DTG was withdrawn for ADRs were assessed. 
Results: A total of 1087 patients was included with mean age of $50 \pm 11.6$ years (14-84), including $71.5 \%$ men and $20.9 \%$ co-infected patients (HBV or HCV). Mean duration of exposure to HIV was 15.7 years. Concerning ARVs combination, DTG was associated with two nucleoside reverse transcriptase inhibitors (NRTI) in 916 (84.3\%) patients with mainly abacavir + lamivudine $\left(\right.$ Kivexa $^{\circledR}+$ Tivicay $^{\circledR}$ ) in $640(58.9 \%)$ patients and emtricitabine + tenofovir (Truvada + Tivicay $\left.{ }^{\circledR}\right)$ in 276 $(25.4 \%)$ patients. There was no significant difference for age, gender or duration of exposure to HIV with patients exposed to DTG without ADRs. However, DTG-induced ADRs were more frequent in co-infected patients for $\mathrm{HBV}$ or $\mathrm{HCV}(\mathrm{OR}=1.71$; IC $95 \%=1.04-2.80)$. The most frequent ADRs were neuropsychiatric $(\mathrm{n}=28 ; 2.6 \%)$, digestive $(\mathrm{n}=21 ; 1.9 \%)$, osteo-articular $(\mathrm{n}=8 ; 0.7 \%)$ and cutaneous $(\mathrm{n}=7 ; 0.6 \%)$. A total of 83 patients $(7.6 \%)$ discontinued DTG due to ADRs, mainly the first month following its introduction.

Conclusion: Our data show a higher rate of DTG withdrawal due to ADRs (7\%) compared to literature data (3\%). Neuropsychiatric ADRs remain the most frequent. Using Nadis ${ }^{\circledR}$ as a data source allows an improvement of ADRs reporting of ARV drugs mainly for new marketed drugs ${ }^{[1,2]}$.

References:

1. de Boer $\mathrm{M}$, et al. Intolerance of dolutegravir containing cART regimens in real life clinical practice. AIDS 2016;30:2831-4

2. Hoffmann $\mathrm{C}$, et al. Higher rates of neuropsychiatric adverse events leading to dolutegravir discontinuation in women and older patients. HIV Medicine. 2016:in press

\section{Adverse Drug Reactions related to Direct Oral Anticoagulant: Patient's Internet Narratives Versus Pharmacovigilance Database}

\section{S. Sadeghi ${ }^{1}$, L. Chebane ${ }^{1}$, J.L. Montastruc ${ }^{1}, \underline{\text { H. Bagheri }}{ }^{1}$ \\ ${ }^{1}$ Service De Pharmacologie Médicale Et Clinique, Centre De Pharmacovigilance, Toulouse, France}

Introduction: Nowadays, Internet is changing the way people learn about illnesses and treatments. Direct Oral Anticoagulants (DOAs) are a new class of drugs supposed to be more practice as they do not require injection or regular haemostasis monitoring. Thus, websites and online discussions could be an interesting source of data on DOA and their Adverse Events (AEs) by allowing patients to exchange freely about their feelings. The aim of the study was to describe the characteristics of their AEs reported by the patients exposed to DOAs and to compare them to those recorded in the French PharmacoVigilance Database (FPVD).

Methods: We performed a retrospective study to collect AEs reported by patients in four websites forums (sante-medecine.fr, allodocteur.fr, meamedica.fr and askapatient.com) from January 2012 to June 2016. For each report, we recorded demographic data, the type and intensity of the AEs and the DOA involved. The same analysis was done in the FPVD for DOA-induced Adverse Drug Reactions (ADRs) from January 2012 to December 2015. AEs and ADRs were then classified in three groups: hemorrhagic effects, thrombotic effects and "others".

Results: A total of 235 AEs were identified in website posts and 7912 ADRs were found in the FPVD. Online discussions included mainly women $(n=49,65 \%)$ and patients aged under $65(n=47,66.2 \%)$. In the FPVD, sex ratio was 1 (2383 women and 2397 men) with a majority of patients aged 65 and older $(\mathrm{n}=3853,80.6 \%)$. More AEs labelled as "others" were found in websites $(\mathrm{n}=205,87.2 \%)$ including mainly arthralgia $(\mathrm{n}=18,8.8 \%)$, asthenia $(\mathrm{n}=17,8.3 \%)$ and headache $(\mathrm{n}=14$, $6.8 \%)$ compared to the FPVD $(\mathrm{n}=2594,26.7 \%)$ where ADRs labelled as "others" were mainly pruritus $(\mathrm{n}=80,2.8 \%)$, asthenia $(\mathrm{n}=72,2.5 \%)$ and acute renal failure $(n=63,2.2 \%)$. Hemorrhagic effects were most frequently recorded in the FPVD $(n=4495,56.8 \%)$ compared to websites $(\mathrm{n}=28,11.9 \%)$. AEs reported in websites were less "serious" $(\mathrm{n}=16$, $21 \%)$ than those recorded in FPVD $(n=3827,80 \%)$.

Conclusion: There are differences between AEs reported in forums and ADRs reported by health professionals. As reported by other studies, data from patients' websites and online discussions would complement those of FPVD and could be used as another source of data in pharmacovigilance to detect ADRs that could affect patients' quality of life.

\section{Measuring the Impact of Product Withdrawals and Other Major Pharmacovigilance Actions on Public Health Burden in the EU: Methodological Considerations}

S. Lane ${ }^{1}$, E. Lynn ${ }^{1,2}$, S. Shakir ${ }^{1,2}$

${ }^{1}$ Drug Safety Research Unit, Southampton, UK, ${ }^{2}$ School of Pharmacy and Biomedical Sciences, University of Portsmouth, Portsmouth, UK

Background: The adoption of pharmacovigilance legislation in 2012 saw major changes to pharmacovigilance processes within the EU [1]. The impact of these changes has not yet been measured, therefore this project has been proposed in line with PRAC and ENCePP strategies for Impact to measure the effect that pharmacovigilance actions have had on public health in terms of serious morbidity and mortality [2-3]. Previous studies have assessed evidence used to support post-marketing product withdrawals; this study will build on previous work to examine changes to the robustness of supporting evidence since adoption of EU pharmacovigilance legislation in 2012 [4-5].

Aim: To promote discussion of methodological considerations regarding impact measurement.

Methods: The aim of the study is to determine the impact of pharmacovigilance activities on public health within the EU. Assessment of evidence supporting pharmacovigilance decisions will follow identification of prescription products suspended or withdrawn from EU market for safety reasons between $01 / 07 / 2012$ and $31 / 12 / 2016$. Studies cited in the evidence will then be used to measure impact on public health, by statistical analyses and impact metrics (e.g. time series regression and population impact number of eliminating a risk factor over time $t$ [PIN-ER- $t$ ] ${ }^{[6]}$ ) and time-point sensitivity analyses. Relative risk of absence together with measured health outcomes or usage figures will be used for this. Further investigations into alternative available products and their risks may also be possible. Other major pharmacovigilance actions providing significant risk minimisation (e.g. contraindications, restrictions) will be investigated in the same manner.

Results: Data collection and identification of valid data sources is ongoing. Databases such as THIN, CPRD and IMS will be used to estimate usage figures. Sources of evidence for pharmacovigilance actions may include scientific and medical literature.

Conclusions: Completion of this study will suggest changes to robustness of evidence supporting pharmacovigilance actions since the adoption of EU legislation in 2012. The main outcome of the study will be 
identification of modelling methods which may contribute to updated ENCePP guidance and used in future impact studies.

Further sources of information/References

1. Montanari-Vergallo G. Recent developments in EU and US pharmacovigilance legislation. J Pharmacovigilance 2013; 1: 1000e105

2. European Medicines Agency. PRAC strategy on measuring the impact of Pharmacovigilance activities. London: EMA; 2016 January 11. (EMA/790863/2015).

3. European Medicines Agency. Mandate of the ENCePP Special Interest Group (SIG) on Measuring the Impact of Pharmacovigilance Activities. London: ENCePP; 2016 July 01. (EMA/43174/2016)

4. Clarke A, Deeks JJ, Shakir S. An assessment of the publicly disseminate evidence of safety used in decisions to withdraw medicinal products from the UK and US markets. Drug Saf 2006; 29:175-81

5. McNaughton R, Huet G, Shakir S. An investigation into drug products withdrawn from the EU market between 2002 and 2011 for safety reasons and the evidence used to support the decision-making. BMJ Open 2014; 4:e004221

6. Verma A, Torun P, Harris E, Edwards R, Gemmell I, Harrison RA, Buchan IE, Davies L, Patterson L, Heller RF. Population impact analysis: A framework for assessing the population impact of a risk or intervention. J. Public Health 2011; 34:83-9

\section{Adverse drug reactions of anticoagulants in Norway in a two year period (2013-2015)}

\author{
$\underline{\text { A. Eek }^{1}}$, B. Oddvar Strøm ${ }^{2}$, G. Bakkeh $\varnothing i^{2}$, H. Stenberg-Nilsen ${ }^{1}$ \\ ${ }^{1}$ Regional Medicines and Pharmacovigilance Center, Oslo University \\ Hospital, Oslo, Norway, ${ }^{2}$ Norwegian Medicines Agency, Oslo, \\ Norway
}

Background and aim: In recent years, several new anticoagulants have been introduced in Norway. The purpose of this study was to improve knowledge of the adverse drug reactions of these drugs in a clinical setting.

Material and methods: All spontaneous adverse drug reaction reports for warfarin, dabigatran, rivaroxaban and apixaban during the period 1 June 2013-31 May 2015 were reviewed. The reports were submitted by health care professionals to the medicines information and pharmacovigilance centers (RELIS). Information from the reports and a supplementary form completed by the reporters, as well as prescription data for anticoagulants from the Norwegian Prescription Database were recorded and analyzed.

Results: The number of reported adverse drug reactions increased during the project period due to increased prescribing of the new anticoagulants (dabigatran, rivaroxaban and apixaban). The most commonly reported adverse drug reactions were hemorrhages, with cerebral bleedings as the most reported. Thrombosis (therapeutic failure), cognitive effects, headache and hair loss were some of the other frequently reported side effects. A large proportion of the patients were 70 years and older. There was slightly more men than women among the patients. The incidence of hypertension, heart diseases and renal failure was higher in the fatal patient group, compared to those who survived. The proportion of reported deaths per 1000 users was highest for rivaroxaban, with decreasing incidence for apixaban, dabigatran and warfarin respectively. The rate of adverse drug reactions classified as severe was $97 \%$ for warfarin, $74 \%$ for dabigatran, $76 \%$ for rivaroxaban and $82 \%$ for apixaban.

Conclusion: Much is still unknown regarding the adverse drug reactions of the new anticoagulants. All substances can induce side effects, including severe reactions. Factors that may increase the risk of side effects include patient age, comorbidity, renal impairment and polypharmacy. All patients treated with anticoagulants should receive regular and thorough follow-up.

\section{HOPIPRAC, PRAC signals validation sentinel system, from a hospital data warhouse}

\author{
L. El Aridi ${ }^{1}$, C. Le Guillou ${ }^{2,3}$, H. Jantzem ${ }^{1}$, A. Happe ${ }^{2}$, \\ D. Carlhant-Kowalski ${ }^{1}$, J. Cauvin ${ }^{2,3}$
}

${ }^{I}$ Centre Régional De Pharmacovigilance, CHRU De Brest, Brest, France, ${ }^{2}$ Département d'information médicale, CHRU de Brest, Brest, France, ${ }^{3}$ LaTIM INSERM-U1101, CHRU de Brest, Brest, France

Introduction: Spontaneous reporting is still a reference device for adverse drug reactions (ADR) collection. However, it is insufficient to ensure a complete and fast collection. Text searching in the electronic medical record opened a new avenue in the ADR collection. Nevertheless, these methods have some limitations. Thus it is possible to consider a systematic reporting by developing technical environment that facilitates the inclusion of all the electronic medical data sources, using keywords and codes, which automates alarm detection once the signal criteria are gathered together for a new patient. Therefore, HOPIPRAC fits into this dynamic of patients' data interrogation in order to rapidly and systematically instruct PRAC (Pharmacovigilance Risk Assessment Committee) signals.

Methods: PRAC signals of the European Medicine Agency must be reinforced by cases reported by the French Pharmacovigilance. Based on a hospital medical records data warehouse, HOPIPRAC, funded by the ANSM, aims to develop a working environment adapted to the pharmacovigilant in order to coordinate all of case detection steps using data from the electronic patient record.

From PRAC signal selected by the pharmacovigilants, sub-warehouses are created using SQL query on the drug and medical terms or diagnostic codes that reflect drug exposure and desired iatrogenic effect. Then the pharmacovigilants use search, navigation and marking functionalities of the sub-warehouse to select the cases for which the imputation between exposure and clinical manifestation strengthen the PRAC signal.

Results: 10 PRAC signals were tested corresponding to 11,824 documents related to 2191 patients and 8558 hospital stays. 8 cases were selected. The interface developed to detect drug exposure takes into account the variability of terms describing this exposure: common names, pharmaceutical specialties and syntactical variations. It relies on a drug database and ORACLE text services. A similar approach is realized to select the ICD-10 codes and the terms describing the iatrogenic effect.

Conclusion: Currently, the project is in an intermediate phase during which the device functionality was validated. Later, the development of search interfaces and event-messaging for recent medical records, coupled with the supply of the warehouse with the prescriptions and laboratory findings should improve the cases detection in a retrospective way as in a prospective way. 


\section{By Selecting Journals for Monitoring by Branches of Medicine, MAHs can Risk Missing Almost Half of Their ICSRs}

\author{
K. Timár-Horváth ${ }^{1}$ \\ ${ }^{1}$ Comfit Europe Ltd., Budapest, Hungary
}

Introduction EMA states ,the marketing authorisation holder should ensure that the literature review includes the use of reference databases that contain the largest reference of articles in relation to the medicinal product properties." [1] MAHs usually interpret this phrase so that they should choose journals from relevant therapeutic fields for their drugs, e.g. journals of cardiology are monitored for medicines in cardiology. However, it is inherent to the nature of pharmacovigilance that we are looking for the unexpected, so that ADRs can turn up anywhere and may not be bound by the borders of medical specializations. Such cases may be published in journals targeted at experts of the prescriber's therapeutic branch, or doctors specialised in treating the given ADR. This implies that with the approach of selecting journals by medical fields, MAHs may risk missing safety data. What is the level of the risk?

Aim: to examine the frequency of publications in which the specialist of a disease is different from the doctor curing the ADR of the therapy used.

Methods: A systematic literature review was conducted in Hungarian local medical journals for the period 1st January-30th June 2015. This consisted of data retrieval from 251 issues of 107 journals. The list of journals was based on the recommendation of the Hungarian NCA. The study focused on articles discussing case reports, aggregated safety data were excluded. The treatments and their ADRs were classified by branches of medicine. They were compared to the specialisation of journals indicated in their impressum to see whether the article reached the specialists of the disease or the ADR.

Results 217 articles met the inclusion criteria of having case reports, out of which 41 articles discussed valid ICSRs. Among them, 18 articles were identified, in which the disease of the patient and the ADR caused by the therapy were treated by doctors working in different fields of medicine, and the specialisation of the journal covered only one of the fields in question. 3 articles [2-4] approached the case from the point of view of the ADR's treatment and were published in journals specialised in the medical field respective to the ADR.

Conclusions Selecting journals for monitoring according to the drug's indications is highly questionable from a pharmacovigilance point of view. To avoid the risk of missing safety data and to comply with the possibility of the unexpected, the best approach for MAHs is to monitor all medical journals.

Further sources of information/References

1. Good Pharmacovigilance Practices (GVP), Module VI, VI.B.1.1.2. Literature report; 9

2. Nagy JE. Csapda a spondylitis ankylopoetica diagnózisában. Magyar Reumatológia 2015; 1: 46

3. Hang D. A hyperlipidaemia követésének komplexitása Parkinsonkórral újonnan diagnosztizált polimorbid beteg esetében. Lege Artis Med 2014; 12: 585-88

4. Lőrincz I, Nagy G, Csapó K, Barna L, Simkó J, Wéber Á. Ritka etiológiájú koronáriaspazmus. Cardiologia Hungarica 2015; 1: 28-31

\section{Big Data of Local Medical Literature Monitoring}

\author{
K. Timár-Horváth ${ }^{1}$ \\ ${ }^{1}$ Comfit Europe Ltd., Budapest, Hungary
}

Introduction: Big data analysis does not exist without having the big data in a structured form. Local medical literature are acknowledged sources of valuable safety data, but have not been integrated in one single database. Moreover, there is no common knowledge or an official list by authorities on local medical journals. Even their selection principles have not been created so far. However, the challenge rather lies in the access to journals. If a journal is accessible online, it is easier to be channelled in a database. From PV point of view, it also matters whether their online sites are updated right after the issue is published. These play a role in what measures are to be taken so to merge all the local journals in a single database for pharmacovigilance monitoring.

Aim: To study the access to local medical journals in six European countries. Methods: The study was conducted on 494 local medical journals from Bulgaria (74), Croatia (40), Hungary (127), Poland (140), Romania (78) and Serbia (35). Local medical journals were included in the study. Medical journals were defined as ones including advertisements of prescription-only drugs, as European law restricts their advertisement to medical professionals only. A journal was considered local if its publishing house has a local address. The following aspects were studied: if any reference databases monitor the journal; if the journal offers online access to their articles, and if they update their website.

Results: $75 \%$ of the journals from the six countries are not included in Embase or Medline. 27\% of the journals are published only in print, $8 \%$ only electronically. $2.42 \%$ do not even have a home page. $44 \%$ are not readable or downloadable online. $38 \%$ of the online accessible journals do not publish their latest issue right after its publication.

Conclusions: All these results suggest that getting safety information from local medical journals is inhibited by obtaining the publications themselves. This is an important issue to solve as currently three out of four local journals are not searchable in international databases, while for example, $82 \%$ of the Hungarian ICSRs in 2016 were published in journals not being part of reference databases. Best practices to find, cooperation of stakeholders to be promoted, encouraging publishing houses to increase their online presence are just a few ideas to work on. As a first step, who will take the role of setting up the list of local medical journals to be monitored?

Further sources of information/References

1. Data retrieved from ComFit's literature database (https://www. comfiteurope.eu/) on 28 April 2017.

\section{Identifying Chemical Substructures Associated with Adverse Drug Reactions using Deep Learning Framework}

\author{
S. Dey ${ }^{1}$, H. Luo ${ }^{1}$, A. Fokoue ${ }^{1}$, J. Hu ${ }^{1}, \underline{\text { P. Zhang }}{ }^{1}$ \\ ${ }^{1}$ IBM T. J. Watson Research Center, Yorktown Heights, USA
}

Introduction: Adverse drug reactions (ADRs) are unintended and harmful reactions caused by normal uses of drugs. Predicting and preventing ADRs 
in early stages of the drug development pipeline can help to enhance drug safety and reduce financial costs.

Aim: We aim to predict ADRs and identify molecular substructures associated with ADRs, so that they can be leveraged in the initial phase of drug design.

Methods: We developed a neural fingerprint method [1] in a simultaneous deep learning framework for ADR prediction without defining the substructures a-priori. The algorithm explores all possible substructures, and summarizes similar structures into final feature representations (i.e., neural fingerprints). We interpreted and analyzed the features to identify which substructures within the drug molecules are specifically related to a particular ADR. Additionally, we used chi-squared test to evaluate their associations and test whether the substructures can help to predict ADRs for new drugs. We compared our method with ten different types of chemical fingerprints (Shortest-path, MACCS, ECFP6 etc. which are implemented in the rCDK package [2]), and used them as features in L2 penalized logistic regression models to assess their performances in ADR prediction based on SIDER database [3].

Results: Our neural fingerprints from the deep learning model achieved an overall accuracy of 0.92 and AUC (the area under the receiver operating characteristic curve) of 0.84 , and outperformed all other pre-defined chemical fingerprints in predicting ADRs. We found that our models performed well on a variety of ADRs in terms of AUC including: (1) skinrelated ADRs such as dermatitis perioral, skin striae and acneiform eruption; (2) metabolic-related ADRs including alkalosis hypokalemic and increased insulin requirement; (3) muscle-related steroid myopathy; and (4) eye related cataract subcapsular. Via feature analysis on drug structures, we identified the specific molecular substructures that are highly associated with these ADRs. We also found literature evidence for the substructures obtained for ADRs, aseptic necrosis and back pain, as two case studies.

Conclusions: The proposed structure-based deep learning models can not only obtain good performance in ADR prediction, but also identify the potential connections between substructures and ADRs in a single datadriven framework without defining substructures a-priori. The deep learning model with feature analysis, substructure identification, and statistical assessment provides a promising solution for identifying risky components within molecular structures and can potentially help to improve drug safety evaluation.

Further sources of information/References:

1. Duvenaud D, Maclaurin D, Iparraguirre J, Bombarell R, Hirzel T, Aspuru-Guzik A, Adams R. Convolutional networks on graphs for learning molecular fingerprints. Adv Neural Inf Process Syst 2015:2224-32

2. Guha R. Chemical informatics functionality in R. J Stat Softw 2007;6:18

3. Kuhn M, Letunic I, Jensen LJ, Bork P. The SIDER database of drugs and side effects. Nucleic Acids Res 2016;44:D1075-9

\section{The WEB-RADR project: Characteristics and Quality of ADR Reports submitted through new reporting tools}

\author{
H. Taavola ${ }^{1}$, O. Caster ${ }^{1,2}$, P. Tregunno ${ }^{3}$, P. Mas ${ }^{4}$, I. Oosterhuis ${ }^{5}$, \\ S. Gama ${ }^{6}$, L. Härmark \\ ${ }^{1}$ Uppsala Monitoring Centre, WHO Collaborating Centre \\ for International Drug Monitoring, Uppsala, Sweden, ${ }^{2}$ Department \\ of Computer and Systems Sciences, Stockholm University, Stockholm, \\ Sweden, ${ }^{3}$ Vigilance and Risk Management of Medicines Division, \\ Medicines and Healthcare Products Regulatory Agency, London, UK,
}

${ }^{4}$ Agency for Medicinal Products and Medical Devices, Zagreb, Croatia, ${ }^{5}$ Netherlands Pharmacovigilance Centre Lareb, 's-Hertogenbosch, the Netherlands, ${ }^{6}$ Novartis Pharma AG, Basel, Switzerland

Background: Spontaneous reporting of suspected ADRs from healthcare professionals (HCPs) and patients is an important source of drug safety information. However, current reporting tools are sometimes perceived as complex and inaccessible. New technologies offer new possibilities to simplify reporting and making it more accessible. Within the WEB-RADR project a mobile app was developed to simplify reporting. Novartis has separately developed a webform (PSI tool) which can also be used on mobile devices. Aim: To evaluate the characteristics and quality of reports submitted by new technologies such as the app and the PSI tool.

Methods: This study includes reports submitted up to September 2016 that (1) were spontaneous, (2) had a single notifier, and (3) were submitted directly by a health care professional or patient. For each organisation separately, the reports from the new tools were compared to a set of reference reports, submitted via conventional means during the same period. The following characteristics were analysed: the proportions of patient reports and reports concerning females, and the median patient age. In addition, a set of 100 app reports and 100 reference reports (for Croatia 37 and 68 reports, respectively) was randomly sampled, stratified by the proportion of patient reports among the reports from new tools. Blinded assessors scored the quality of reports in this subset using a tool called ClinDoc [1], and the proportion of reports of at least moderate quality was compared.

Results: A significantly higher proportion of app reports were submitted by patients in UK and Croatia $(\mathrm{p}<0.01)$, whereas in Netherlands and for Novartis tool the difference was small and non-significant. The proportion of female patients and the median age of patients among app reports was relatively similar to the reference group in all countries. For the Novartis data, it was reported more on women $(\mathrm{p}<0.01)$ and slightly younger patients $(\mathrm{p}<0.05)$. The proportion of reports of at least moderate quality for the app was high in both groups, except for Novartis, but relatively lower for app reports.

Conclusion: New reporting tools such as the WEB-RADR app and the PSI tool offer a complementary route of spontaneous reporting. Patient demographics are similar to conventional reporting routes, and report quality is sufficient despite a simplified reporting form. The addition of new reporting forms is important to attract more reporters. The data shows that the app has managed to attract patients as reporters to a larger extent than conventional reporting routes.

\section{Further sources of information/References:}

1. Oosterhuis, I. et al. Development and validity testing of a clinical documentation tool to assess Individual Case Safety Reports in an international setting. Pharmacoepidemiol Drug Saf 2016; 25(Suppl 3):417

Table 1. Differences in proportion of patient reports and propotion of reports with moderat quality between new and conventional reporting tools.

\begin{tabular}{|l|l|l|l|}
\hline $\begin{array}{l}\text { Proportion of patient } \\
\text { reports }\end{array}$ & New (\%) & Conventional (\%) & $p$ \\
\hline UK & 28 & 18 & $p<0.01$ \\
\hline HR & 32 & 7 & $p<0.01$ \\
\hline NL & 60 & 57 & $p=0.5$ \\
\hline Novartis & 0.3 & 0.6 & $p>0.05$ \\
\hline $\begin{array}{l}\text { Proportion of reports } \\
\text { with moderate quality }\end{array}$ & & & \\
\hline UK & 83 & 92 & $p=0.08$ \\
\hline HR & 78 & 78 & $p=1.0$ \\
\hline NL & 85 & 98 & $p<0.01$ \\
\hline Novartis & 31 & 33 & $p=0.79$ \\
\hline
\end{tabular}


Disclosure: The research leading to these results was conducted as part of the WEB-RADR consortium, (http://webradr.eu) which is a public-private partnership coordinated by the Medicines and Healthcare products Regulatory Agency. The WEB-RADR project has received support from the Innovative Medicine Initiative Joint Undertaking (www.imi.europa.eu) under Grant Agreement $n^{\circ} 115,632$, resources of which are composed of financial contribution from the European Union's Seventh Framework Programme (FP7/2007-2013) and EFPIA companies' in kind contribution. The views expressed are those of the authors only.

\section{Three cases of seizure with nivolumab: a new class of adverse effects for the anti-PD-1 immunotherapy?}

\author{
V. Chawadronow ${ }^{1}$, C. Maucorps ${ }^{1}$, L. Dubuc-Lepetit ${ }^{2}$, A. Martin ${ }^{3}$, \\ M. Sassier ${ }^{1}$, S. Fedrizzi ${ }^{1}$, A. Coquerel ${ }^{1}$
}

${ }^{1}$ Regional Pharmacovigilance Centre, Department of Pharmacology, University Hospital, Caen, France, ${ }^{2}$ Department of Neurology, Memorial France-United States Hospital, Saint lô, France,

${ }^{3}$ Department of Pharmacy, Memorial France-United States Hospital, Saint lo, France

Introduction: Immunotherapy is a recent and major advance in the treatment of cancers. The current drugs are monoclonal antibodies that restore immunity by blocking lymphocyte inhibitory pathways, particularly the PD-1 (programed-death-1) receptor. Such therapies could lead to many adverse reactions but some side effects are still not known or not explained.

Aim: We describe 3 cases of seizures during nivolumab treatments spontaneously reported to our pharmacovigilance centre.

Method: These three cases were medically analyzed (including Computing Tomography [CT] scan and Magnetic Resonance Imaging [MRI] and Electroencephalography [EEG]). Differential diagnoses explaining seizures like brain tumors, stroke or metastasis were excluded. Then we confronted our data to the French and international database of pharmacovigilance $[\mathrm{PV}]$ and to literature (PUBMED).

Results: The 3 cases concerned patients treated with nivolumab at the same dose of $3 \mathrm{mg} / \mathrm{kg}$ every 2 weeks. Two of them were 59 and 70 years old and were diagnosed with non-small cell lung cancer. The third was a 51 years old man included in a clinical trial for a kidney cancer. The first patient had a seizure after his 4th cycle that re-offend despite treatment with levetiracetam. The second patient presented also a seizure after his 15th cycle. A few days later the patient died after a respiratory distress syndrome. For the third patient successive convulsions were observed from the 6th cycle. Various treatments have been tried to avoid recurrence. Only the withdrawal of the nivolumab enabled the resolution. In all cases, the CT scan and MRI did excluded brain tumors. Only the first patient MRI showed slight inflammation in the peri-ventricular white matter. Usual and opportunistic infections were excluded. Epileptic symptoms always began by myoclonus and worsened with status epilepticus or generalized seizures. In the literature, we found a case of seizure with nivolumab after the second cycle for a glioma ${ }^{1}$. The disease itself could explain or promote this effect. Another case of seizure with another antiPD-1 (lambrolizumab) ${ }^{2}$ is described. After the 4th cycle, myoclonus started in arms. Two cycles later a general seizure occurred. Myoclonic seizures reappeared and persisted 3 weeks later. Only the withdrawal of lambrolizumab allowed the seizures resolution. We did not found similar cases in PV database. An immune origin is suspected.

Conclusion: Seizures seem to be an hazardous serious effect during a treatment by anti-PD-1 monoclonal antibodies. These seizures begin as myoclonus and seem to be drug resistant, imposing the treatment discontinuation.

Further sources of information/References:

1. $1 \mathrm{Zhu} \mathrm{X}$, et al. Severe cerebral edema following nivolumab treatment for pediatric glioblastoma: case report. J Neurosurg Pediatr. 2016; $18: 1-5$

2. 2 Mandel JJ., et al. Lambrolizumab induced central nervous system (CNS) toxicity. J Neurol Sci 2014; 344: 229-231

\section{Eculizumab therapy and fatal seizures: about four cases}

Y. Cazaubon ${ }^{1}$, B. Azzouz ${ }^{1}$, Z. Djerada ${ }^{1}, \underline{\text { T. } \text { Trenque }^{1}}$

${ }^{\text {I}}$ Pharmacovigilance Regional Center, Department of Medical Pharmacology and Toxicology, Reims University Hospital, Reims, France

Background: Atypical hemolytic-uremic syndrome (aHUS) is a rare, severe and life-threatening disease. It is characterized by a thrombotic microangiopathy, hemolytic anemia, thrombocytopenia, and renal failure. Eculizumab is a monoclonal antibody that binds with high affinity to C5 and inhibits the generation of inflammatory, prothrombotic, and lytic terminal complements component. Eculizumab was first approved for the treatment of patients with paroxysmal nocturnal hemoglobinuria and then it has successfully been used in patients with aHUS, and is becoming the first-line treatment.

Aim: We report cases of eculizumab use in adult patients for treating aHUS who are developing neurological complications with seizures and fatal outcome.

Methods: A retrospective analysis was performed in Eudravigilance database by using preferred term "seizure" and in our Pharmacovigilance Regional database. Demographic data, creatininemia, thrombopenia, anemia, duration between eculizumab treatment and death (TDe) and between diagnosis and time to take care of aHUS (DiT) were recorded in our database.

Results: In our database, four patients $(2 \mathrm{~F} / 2 \mathrm{M})$ were collected with a fatal issue for all. Mean age was 56.3 [25-81]. Before aHUS, two of four patients were in normal range for creatininemia $(45-115 \mu \mathrm{M})$. During aHUS diagnosis, creatininemia was $1.3 \mathrm{~N}$ to $2.8 \mathrm{~N}$, hemoglobin was 6.8 to $8.8 \mathrm{~g} / \mathrm{dL}$ (N: $12-17 \mathrm{~g} / \mathrm{dL}$ ) and thrombocyte was 13 to $23 \mathrm{G} / \mathrm{L}$ (N: $150-450$ $\mathrm{G} / \mathrm{L}$ ). Dosage regimen was $900 \mathrm{mg}$ per week. Mean DiT was 1.8 days [0-7] and mean TDe was 53.8 days [17-87]. In Eudravigilance, 96 cases were related with seizures. Among these cases, 8 were fatal including our regional cases, 18 not recovered, 20 resolved, 3 resolved with sequelae, 4 recovering and 43 unknown.

Conclusion: To our knowledge, this is the first report undergoing the possible link between eculizumab therapy and fatal seizures. For our cases, the mean DiT was short (1.8 days) and seizures with fatal outcome. Another point to highlight is also the short duration between eculizumab initiation and death.

Discussion: Pape et al. (1) showed that in severe HUS cases which progress rapidly with multiple organ involvement, late treatment with eculizumab seemed to be less benefit. They speculated that prophylactic eculizumab therapy before development of neurological symptoms could be advantageous. The involvement of eculizumab administration with neurological alterations remains questionable. More detailed studies are needed. 


\section{Further sources of information/References}

1. Pape L, Hartmann H, Bange FC, Suerbaum S, Bueltmann E, Ahlenstiel-Grunow T. Eculizumab in Typical Hemolytic Uremic Syndrome (HUS) With Neurological Involvement. Medicine (Baltimore). 2015 Jun;94:e1000

\section{Developing a Signal Detection and Prioritisation Triage Logic for the Korea Adverse Event Reporting System-foreign Database: Results of Pilot Study}

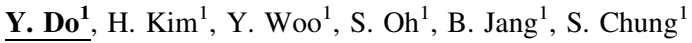 \\ ${ }^{1}$ Korea Institute of Drug Safety \& Risk Management, Anyang-si, \\ South Korea
}

Background: The Korea Adverse Event Reporting System-foreign (KAERS-foreign) was launched on 21 August 2014 in the Republic of Korea in order to collect adverse reaction reports relating to serious events occurring outside Korean territory.

Various quantitative signal detection methodologies have been devised and tested which extract information about drug safety [1] from the various spontaneous-reporting system databases; however, there is no standard methodology for signal detection in terms of data mining [2]. It has been recommended that a quantitative signal detection algorithm should be developed to suit the organisational goals and the resources available. [3] In addition, prioritising detected signals at a local level for regulatory purposes is another challenge: public health aspects need to be considered [4-5].

Aim: To develop a signal detection and prioritisation triage logic that accords with Korea Institute of Drug Safety and Risk Management's safety information management requirements, utilising the KAERS-foreign database.

Methods: A reconstructed version of the KAERS-foreign database as of 31 December 2014 was utilised for the analysis. Only the final follow-up individual-case safety reports were included and the nullified reports were excluded. The substance names for drugs were coded using the WHODrug Dictionary Enhanced (WHO-DDE) and adverse event names were coded using the Medical Dictionary for Regulatory Activities $\left(\right.$ MedDRA $\left.^{\circledR}\right) 18.0$ preferred terms (PT). Proportional Reporting Ratio (PRR) and Chi-square () values were calculated for all possible drug-event combinations using Empirica ${ }^{\mathrm{TM}}$ Signal \& Topics 8.0 and the thresholds of number of cases $(\mathrm{N}) \geq 5, \mathrm{PRR} \geq 2$ and $\geq 4$ were applied. The monthly increase in PRR value for each combination [6] was calculated using SAS $^{\circledR}$ 9.3. The local list of Designated Medical Events (DMEs) and the strength of signal (i.e. PRR value) were considered when the subsequent selection of the combinations took place

Results: A total of 499 drug-event combinations were identified after applying the criterion of ' $>50 \%$ increase in PRR values' [6]. These combinations comprise 220 substance names and 341 adverse events; sixteen of the latter had been included in the local DME list. After a review of the local labels of the selected combinations, some of these were then considered to be potential new signals.

Conclusions: A manageable number of combinations have been were yielded by the developed procedure. However, there is the possibility that some potentially relevant signals were excluded. The developed procedure, with its triage logic was intended to integrate the signal detection and prioritisation steps with regards to signal management. We plan to improve this procedure by including a systematic decision-making step.

\section{Further sources of information/References}

1. Shibata A, Hauben M. Pharmacovigilance, signal detection and signal intelligence overview. Information Fusion (FUSION), 2011
Proceedings of the 14th International Conference on 2011 Jul 5 (pp. 1-7). IEEE

2. Haubenand M, Norén GN. A decade of data mining and still counting. Drug Saf 2010;33:527-34

3. Wisniewski AF, Bate A, Bousquet C, Brueckner A, Candore G, Juhlin K, Macia-Martinez MA, Manlik K, Quarcoo N, Seabroke S, Slattery J. Good signal detection practices: evidence from IMI PROTECT. Drug Saf 2016;39:469-90

4. Levitan B, Yee CL, Russo L, Bayney R, Thomas AP, Klincewicz SL. A model for decision support in signal triage. Drug Saf 2008;31:727-35

5. Ståhl M, Lindquist M, Edwards IR, Brown EG. Introducing triage logic as a new strategy for the detection of signals in the WHO Drug Monitoring Database. Drug Saf 2004;13:355-63

6. Lerch M, Nowicki P, Manlik K, Wirsching G. Statistical signal detection as a routine pharmacovigilance practice: effects of periodicity and resignalling criteria on quality and workload. Drug Saf 2015;38:1219-31

\section{Applying DoTS Adverse Drug Reactions Classification to Reports from Hospital Spontaneous Stimulated Reporting}

A. M. Farcas ${ }^{1}$, N.B. Bulik ${ }^{1}$, A. Popa ${ }^{1}$, A. Mensah Achampong ${ }^{1}$, C. Bucsa ${ }^{1}$, C. Mogosan ${ }^{1}$

${ }^{1}$ Drug Information Research Center, University of Medicine and Pharmacy, Cluj-Napoca, Romania

Background: Evaluating adverse drug reactions (ADRs) detected in clinical practice provides important insights for their future careful monitoring and prevention. Although the Rawlins and Thomson type A and B ADRs classification [1] remains the most widely used, a more recent classification, offering a three dimensional approach considering dose, time and susceptibility (DoTS), was proposed for improved patient care [2].

Objective: To assess ADRs using the new and more complex DoTS classification, aside the Rawlins and Thomson classification.

Methods: 251 ADRs detected in hospitalized patients by spontaneous stimulated reporting and stored in our database were evaluated by a panel of 4 pharmacists experienced in pharmacovigilance ( 1 senior and 3 junior). The information available for evaluation was patient's characteristics (age, sex), including co-morbidity, renal and hepatic function, history of alcohol intake, previous ADRs or known allergies, co-medication, clinical and biological data, ADR description, including causality, seriousness, treatment and outcome, response to dechallenge and rechallenge, and information on suspected drug (dose and treatment duration). Except time, for each DoTS criteria one or more subtypes were chosen based on the agreement of the evaluators.

Results: The ADRs affected mostly women in our cohort (189 ADRs out of 251) and the average patients' age was 63 [24-89]. 215 ADRs were classified as type A and 36 as type B according to Rawlins and Thomson classification. Using the DoTS classification the most frequent associations were collateral/ intermediate reactions (102 ADRs, 41\%), followed by toxic/ intermediate reactions (48 ADRs, 19\%), collateral/early reactions (40 ADRs, 16\%), collateral/ late reaction (27 ADRs, 11\%) and hypersusceptibility/ intermediate reactions (17 ADRs, 7\%). The most frequent susceptibility parameter was age (102 cases, $41 \%)$, followed by drug-drug interactions (in 65 cases, 26\%), sex (49 cases, 20\%) and renal or 
hepatic insufficiency (in 25 cases, 10\%). We have found more than one susceptibility factor in 95 of the cases.

Conclusions: Although application of this classification requires good training and well documented cases, it allows for the identification of high and low risk patients, observance of the time course of a reaction and therefore appropriate monitoring, with the aim of ADRs prevention and risk minimization.

Further sources of information/References:

1. Rawlins MD, Thompson JW. Pathogenesis of adverse drug reactions. In: Davies DM, ed. Textbook of adverse drug reactions. Oxford: Oxford University Press, 1977: 10

2. Aronson JK, Ferner RE. Joining the DoTS: new approach to classifying adverse drug reactions. BMJ 2003;327:1222-5

\section{Years Medication Error Reporting by German Pharmacists: A Disproportionality Analysis}

\author{
M. Ganso' ${ }^{1}$, A. Said ${ }^{1}$, M. Schulz \\ ${ }^{1}$ Drug Commission of German Pharmacists, Federal Union \\ of German Associations of Pharmacists, Berlin, Germany
}

Introduction: When the new EU pharmacovigilance legislative came into force in 2012, adverse drug reactions (ADR) also encompasses medication errors (ME). As a national pharmacovigilance centre, the Drug Commission of German Pharmacists (AMK) collects and evaluates spontaneous reports of suspected risks associated with medicinal products, provided by German primary and secondary care pharmacists. All reports are handled according to the so-called graduated plan (Stufenplan according to $§ 63$ Medicinal Products Act). The AMK collaborates with the national competent authorities as well as marketing authorization holders to minimize potential harm to patients.

Aim: To generate signals by analysing disproportionalities of reported ME associated with different ATC $5^{\text {th }}$ level codes.

Methods: We analyzed our database for the period January 2013 to December 2016. We considered reports for both quality defects and ADRs. We classified risks according to internal standards. The reporting odds ratios (ROR) with the $95 \%$ confidence intervals (95\% CI) were calculated on the 5th level of the ATC code, German version. We used MS Excel ${ }^{\circledR}$ for all calculations. A ROR $>2$ with a $95 \% \mathrm{CI}>1$ and at least four reported cases were defined as a signal [1].

Results: During the 4-year period, we received 31.115 spontaneous reports to medicinal products and 830 of them (2.7\%) were reported as ME. Reporters mostly used quality defect forms for potential ME, whereas errors that had led to an ADR were preferably reported on ADR forms. Compared with all ATC $5^{\text {th }}$ level codes reported, 38 different ATC 5th level codes were disproportionally associated with ME and met the criteria of a signal. Amongst them, coagulation factor IX, II, VII and X in combination (ROR: 27.6, 95\% CI 9.5-79.6), mitomycine (ROR: $16.3,95 \%$ CI 5.0-53.0), insulin lispro 'Mix' (ROR: $12.2,95 \%$ CI 4.4-33.7), dexamethasone and antiinfectives (ROR: $11.8,95 \%$ CI 5.6-25.2), follitropin alfa (ROR: 10.8, 95\% CI 4.0-29.3), aclidinium bromide (ROR: 10.5, 95\% CI 8.0-13.9), ciclesonide (ROR: 10.2, 95\% CI 3.8-27.5), formoterol/beclometasone (ROR: 9.2, 95\% CI 6.6-12.8), peginterferon alfa $2 \mathrm{~b}$ (ROR: 8.2, 95\% CI 3.4-19.8) and leuproreline (ROR: 6.4, 95\% CI 2.2-18.5) revealed the highest measures of disproportionality.

Conclusions: Disproportionality analyses of German pharmacists' spontaneous reports of suspected risks associated with medicinal products identified ATC 5th level codes significantly associated with ME. These signals require validation, however.

\section{Further sources of information/References}

1. van Puijenbroek EP, Bate A, Leufkens HG, Lindquist M, Orre R, Egberts AC. A comparison of measures of disproportionality for signal detection in spontaneous reporting systems for adverse drug reactions. Pharmacoepidemiol Drug Saf 2002; 11: 3-10

\section{Contraceptive and pregnancy testing requirements in clinical trials from French academic sponsors}

S. Crepin ${ }^{1}$, C. Roussillon ${ }^{2}$, M. Gavard ${ }^{3}$, C. Muller ${ }^{4}$,

A. Chilloleau ${ }^{5}$, T. Ouk ${ }^{6}$, C. Mouchel ${ }^{7}$, L. Peyro Saint Paul ${ }^{8}$, N. Petitpain ${ }^{9}$, S. Duranton ${ }^{10}$, P. Olivier ${ }^{11}$, A.1. Colin ${ }^{11}$, M.P. Franceschi ${ }^{12}$, C. Lefeuvre ${ }^{7}$

${ }^{1}$ Unité de Vigilance des Essais Cliniques, Service de Pharmacologie et Toxicologie-Pharmacovigilance, Limoges, FRANCE, ${ }^{2}$ Direction de la Recherche Clinique et de l'Innovation, Sécurité et Vigilance des Etudes Cliniques, Bordeaux, France, ${ }^{3}$ Vigilance des Essais Cliniques, Grenoble, France, ${ }^{4}$ Vigilance des Essais Cliniques, Hôpitaux civils de Strasbourg, Strasbourg, France, ${ }^{5}$ Direction de la Recherche, Département Promotion, Cellule Vigilances, Nantes, France, ${ }^{6}$ Cellule Vigilance-Fédération de Recherche Clinique Département de la Recherche en Santé, Lille, France, ${ }^{7}$ Centre d'Investigation Clinique INSERM 1414, Service de Pharmacologie, Université de Rennes 1, Rennes, France, ${ }^{8}$ Pharmacovigilance des Essais Cliniques, Centre Régional de Pharmacovigilance, Caen, France, ${ }^{9}$ Centre Régional de Pharmacovigilance, Nancy, France, ${ }^{10}$ Vigilance des Essais Cliniques, Direction de la Recherche Clinique et de l'Innovation, Poitiers, France, ${ }^{11}$ Vigilance des Essais Cliniques, Service de Pharmacologie Médicale et Clinique \& Direction de la Recherche, Toulouse, France, ${ }^{12}$ Vigilance Essais Cliniques, Nîmes, France

Background: Patients of reproductive potential participate in all phases of clinical trials $(\mathrm{CT})$. It is then necessary to limit potential embryofetal risks by setting up contraception use and pregnancy testing. Guidelines exist such as M3(R2) guideline from the International Conference of Harmonization and more recently recommendations related to contraception and pregnancy testing in CT from the Clinical Trial Facilitation Group (CTFG) of the European Heads of Medicines Agency.

Objective/Aim: To describe the requirements for contraception and pregnancy testing in French academic sponsors' CT.

Methods: All drug CT including women and men of childbearing potential approved by ANSM (French competent authority) from 2015 and sponsored by 10 French academic sponsors were included. Data collection was completed by sponsors' vigilant staff with a standardized questionnaire.

Results: Data from 49 CT were compiled. $94 \%$ were phase $2-4$ CT. The length of treatment with investigational medicinal product (IMP) varied widely from a unique administration to 2 years. Apart from placebo, 74 IMP were used with $31 \%$ of them belonging to the ATC class "antineoplastic and immunomodulating agents". $28 \%$ of IMP were considered as teratogenic (or suspected of teratogenicity) and $47 \%$ as fetotoxic (or suspected of fetotoxicity) by the sponsors. Contraception at inclusion was requested for $61 \%$ of $\mathrm{CT}$ regardless for the gender, $59 \%$ for women of childbearing potential and $20 \%$ for men of childbearing potential. In CT with contraception requirements, effective contraception was requested in $72 \%$ and highly effective contraception in $24 \%$. By contrast, details on authorized contraception were provided in only $47 \%$. Contraception was requested for $2 / 3$ of IMP considered as teratogenic and for $70 \%$ of IMP considered as fetotoxic. 
Pregnancy testing was requested at inclusion in $61 \%$ of CT and during CT in $16 \%$. For CT with teratogenic IMP, pregnancy test at inclusion was requested in $80 \%$ and for CT with fetotoxic IMP in $70 \%$. In $80 \%$ of the $\mathrm{CT}$, no requirement on how to handle a pregnancy was provided in the protocol. In $22 \%$, the competent authority or the ethic committee requested modification in contraceptive or pregnancy testing requirements. Conclusion: Pregnancy testing and contraception requirements were not consistently addressed in CT enrolling patients of childbearing potential. The safe inclusion of a person of childbearing potential should be adapted based on the assessment of the potential risks of IMP. The recent recommendations from the CTFG should help sponsor to meet regulatory expectations.

\section{Suspected adverse reactions to alpha lipoic acid- containing food supplements: analysis of the Italian Surveillance System of Natural Health Products}

\author{
F. Menniti-Ippolito ${ }^{1}$, R. Da Cas ${ }^{1}$, P. Moro ${ }^{2}$, G. Mazzanti ${ }^{3}$, \\ F. De Ponti ${ }^{4}$, A. Girardi ${ }^{4}$, E. Raschi ${ }^{4}$
}

${ }^{1}$ Italian National Institute Of Health, Rome, Italy, ${ }^{2}$ Niguarda Ca' Granda Hospital, Poison Control Centre, Milan, Italy, ${ }^{3}$ Department of Physiology and Pharmacology, Sapienza, University of Rome, Rome, Italy, ${ }^{4}$ Pharmacology Unit, Department of Medical and Surgical Sciences, Alma Mater Studiorum, University of Bologna, Bologna, Italy

Background: Products containing alpha lipoic acid (ALA) are marketed as food supplements, thus, not claiming clinical indications and not intended for therapeutic purposes. However, they are largely used for the treatment of diabetic neuropathy, and their use is expanding in different diseases as supportive therapy, especially because of the supposed antioxidant properties of ALA against mitochondrial dysfunction [1]. Due to their regulatory status their benefit/risk profile safety profile is unknown.

Aim: To describe suspected adverse reactions (ARs) associated with food supplements containing ALA.

Methods: Spontaneous reports of suspected ARs to food supplements were collected within the Italian Surveillance System of Natural Health Products, coordinated by the National Institute of Health, in collaboration with the Italian Medicines Agency and the Ministry of Health. The analyses took into account data collected between April 2002 and March 2017; diagnoses were coded according to the Medical Dictionary for Regulatory Activities (MedDRA version 4).

Results: Eighty-five reports concerning 160 suspected ARs to ALA food supplements were collected during the study period (5\% out of 1589 total ARs entered in the database). Neuropathic pain, carpal tunnel syndrome and low back pain were the main reasons for use. The mean age was 60 years, $69 \%$ of ARs occurred in females. Reported events mainly consisted in 92 allergy and/or hypersensitivity symptoms (58\% of reactions) and hypoglycemia in 13 cases (8\%). In eight of the 13 patients with hypoglycemia, autoimmune insulin syndrome (also known as Hirata disease) was diagnosed. Thirty-three patients (39\%) needed hospitalisation, in 41 (48\%) concomitant drugs were reported. Dechallenge was positive in 51 cases; rechallenge in 7 cases. The time-to-onset was 1 day in 35 cases, and within 1 week in 52 patients.

Conclusions: The Italian Surveillance System of suspected ARs to Natural Health Products proved to be a useful tool to monitor the safety of food supplements containing ALA in a National context. The variegate uses, without proven efficacy, together with the large proportion of clinically-significant episodes of hypersensitivity reactions and hypoglycaemia, albeit known from the literature [2], call for continuous monitoring by clinicians and pharmacists, especially considering that ALA food supplements may be used as self-medications.

Further sources of information/References:

DOIs not required

1. Koufaki M. et al. Therapeutic applications of lipoic acid: a patent review (2011-2014). Expert Opin Ther Pat. 2014 Sept;24(9):9931005.

2. Gullo D. et al. Insulin autoimmune syndrome (Hirata Disease) in European Caucasians taking $\alpha$-lipoic acid. Clin Endocrinol (Oxf). 2014 Aug;81(2):204-9.

\section{Suspected adverse reactions to herbal dietary supplements for weight loss collected by the Italian surveillance system on natural products}

\author{
$\underline{\text { F. Menniti-Ippolito }}{ }^{1}$, A. Vitalone ${ }^{2}$, R. Da Cas ${ }^{1}$, G. Mazzanti $^{2}$
}

${ }^{1}$ Italian National Institute Of Health, Rome, Italy, ${ }^{2}$ Dept. Physiology and Pharmacology "Vittorio Erspamer", Sapienza University of Rome, Rome, Italy

Introduction: Obesity and overweight are growing public health problems all over the world and their treatment is becoming of great importance also considering the limited number of drugs approved for this indication. Many people use herbal dietary supplements (HDS) for weight loss, even though their benefit/risk profile is not known.

Aim: To analyze spontaneous reports of suspected adverse reactions to HDS or galenic formulations containing herbals used for weight loss purposes.

Methods: In Italy since 2002 a specific Phytosurveillance system was set up. The surveillance is based on the collection and evaluation of spontaneous reports of suspected adverse reactions (ARs) to: food supplements; herbal preparations and galenic formulations; homeopathic medicines. A Scientific Committee evaluates serious reactions. The activities are coordinated by the National Institute of Health in collaboration with the Italian Medicines Agency and the Ministry of Health.

Results: Up to March 2017, 1549 reports of ARs to the above mentioned products have been collected; here we describe those associated with herbal products used for weight loss, received from July 2010 to December 2016. Data up to June 2010 have been previously published [1]. The ARs were analyzed and causality assessment was performed using the WHO-UMC scale.

Forty seven ARs to weight loss products were collected. Women were involved in $83 \%$ of reports. The herbal products involved in the reactions were multicomponent (2-14 components) in more than $80 \%$ of cases; three galenic preparations and one homeopathic medicine were also involved. The reactions affected mainly the cardiovascular system, the liver, the skin, the digestive system and the central nervous system. In about $28 \%$ of cases other medications were reported, while concomitant conditions were present in $23 \%$ of the reports. Most of ARs were serious and required hospitalization.

Conclusions. Present results confirm the safety issues in the use of herbal products for weight loss. Herbal dietary supplements are easily available on the market and consumers use them as self medication, unaware of the risk associated with their use and of lack of evidence of their efficacy. Encouraging spontaneous reporting can contribute to improve awareness among health personnel and patients about the risk profile of these remedies. 


\section{Further sources of information/References}

1. Vitalone A et al. Suspected adverse reactions associated with herbal products used for weight loss: a case series reported to the Italian National Institute of Health, Eur J Clin Pharmacol. 2011 Mar;67(3):215-24.

\section{An example of robust Pharmacovigilance practice: a safety signal of acute kidney injury with combination therapy of piperacillin/tazobactam and vancomycin}

\author{
S. Harmeyer ${ }^{1}$, D. Hewlett ${ }^{1}$, M. Delgado ${ }^{1}$, W. Wang ${ }^{1}$, \\ M. Younus ${ }^{1}$, L. Reich ${ }^{1}$ \\ ${ }^{1}$ Pfizer Inc, Sandwich, UK
}

Introduction: Pfizer maintains a robust pharmacovigilance (PV) program (including collection and processing of adverse event (AE) reports and advanced signal detection processes) to ensure timely presentation of essential new safety information through product labelling. Piperacillin/tazobactam (P/T) and vancomycin is a commonly used combination for empiric therapy of life threatening infections. Although acute kidney injury (AKI) has been associated with both drugs individually, a new safety signal suggested that this combination therapy may have a synergistic effect on this risk.

Aim: To present how Pfizer identified and evaluated a new safety signal of $\mathrm{AKI}$ in connection with combination therapy of $\mathrm{P} / \mathrm{T}$ - vancomycin using Pfizer PV processes and the resulting mitigation measure taken for this new safety risk.

Method: Pfizer learned about a new safety signal (AKI) associated with combination therapy from a poster presented at a Congress held in December 2013. An in-depth evaluation of this signal included a comprehensive review of published literature and other data presented at scientific conferences, analysis of spontaneous cases identified from the Pfizer and FDA (FAERS) safety databases and consultations with internal experts.

Results: Thirty three studies were identified which suggested an increased risk of AKI with combination therapy, and a marked increase of relevant $\mathrm{P} / \mathrm{T}$ cases was detected from around 2009. Evaluation of the entirety of the data led to the inclusion of three proactive updates to the P/T Company Core Safety Information (CCSI) and local labels to further mitigate the risk of AKI in patients using combination therapy.

Conclusion: The increase in AKI reports with P/T - vancomycin combination therapy may be a result of updates to practice guidelines, as for example, the American Thoracic Society (in 2009) or the Infectious Disease Society of America (in 2009) on the management of staphylococcal infections, which encourages vancomycin doses to exceed manufacturer's recommended maximums in order to achieve trough levels of 15 $\mathrm{mg} / \mathrm{L}$. Although the cause for the increase in AKI associated with P/Tvancomycin combination therapy may be multifaceted, it is our opinion that higher dosing recommendations for vancomycin has been a major factor as reflected in the proactive updates of the CCSI.

\section{Reducing prescribing errors associated with inpatient electronic prescribing systems: an investigation of pharmacist interventions to prevent prescribing errors}

\author{
F. Alshahrani ${ }^{1}$, J.F. Marriott ${ }^{1}$, A.R. Cox $^{1}$ \\ ${ }^{1}$ School of Pharmacy, University of Birmingham, Birmingham, UK
}

Introduction: Interventions and advice in the prescribing process provided by pharmacists have been considered a potentially valuable tool to rationalise drug therapy and reduce medication prescribing errors. It is important to understand the incidence and types of medication prescribing errors in order to implement preventive strategies.

Aim: The aim of this study was to examine the number, types, and severity of prescribing errors documented by hospital pharmacists within an electronic prescribing system and their role in identifying and preventing prescribing errors.

Setting: A large teaching hospital in the UK operating an electronic prescribing information and patient management system, which includes electronic pharmacist intervention functionality.

Methods: A prospective one month audit of interventions undertaken by pharmacists as part of their routine practice was analysed to detect prescribing errors in inpatient electronic medication orders. Prescribing error data collected included: medication and medication class (according to British National Formulary categories), type of error, prescribing stage (on admission, during stay, on discharge) and clinical significance. Error classifications were modified from the EQUIP study [1] to suit electronic prescribing errors; the potential severity of errors was pharmacist selfassessed using the EQUIP study severity scale.

Results: Pharmacists logged 1629 interventions on the electronic prescribing system over the month. From these interventions, 1481 (91\%) prescribing errors were identified and rectified before reaching patients. The most common drug classes involved were cardiovascular system $(20.5 \%)$, antibacterial (15.7\%) and central nervous system (14.5\%). Errors most often occurred within general medicine $(61 \%)$. The most common error types identified were omission of medications (42.6\%), dosing errors $(18.2 \%)$ and data entry error (15.7\%) — collectively accounting for more than $75 \%$ of all prescribing errors. Errors were classified as minor (68.4\%), significant $(26.3 \%)$, serious $(5 \%)$ and potentially lethal $(0.1 \%)$. Prescribing errors on admission to hospital were the largest group $(\mathrm{n}=837,56.5 \%)($ Table 1$)$.

Conclusions: Pharmacists detected a significant number of important prescribing errors within this e-prescribing system. Electronic prescribing systems do not eliminate all error. The majority of pharmacist interventions were acted upon, confirming their vital role in preventing prescribing errors potentially harming patients, particularly on admission to hospital. Future studies should evaluate the potential to further reduce prescribing errors by optimising the pharmacist role within electronic prescribing systems and the healthcare team.

\section{Further sources of information/References}

1. Dornan T, Ashcroft D, Heathfield H, Lewis P, Miles J, Taylor D, Tully M, Wass V. (2009) An in-depth investigation into causes of 
prescribing errors by foundation trainees in relation to their medical education: EQUIP study. Final report to the General Medical Council. University of Manchester: School of Pharmacy and Pharmaceutical Sciences and School of Medicine.: eScholarID:83866 URL: www. gmc-uk.org/FINAL_Report_prevalence_and_causes_of_prescribing_errors.pdf_28935150.pdf (Accessed 2017 April 2)

Table 1: Error rates by prescribing stage.

\begin{tabular}{|l|l|}
\hline Stage & $\begin{array}{l}\text { Number of } \\
\text { occurrences }\end{array}$ \\
\hline On admission & 837 \\
\hline During stay & 425 \\
\hline On discharge & 219 \\
\hline Total & 1481 \\
\hline
\end{tabular}

\section{The RE-AIM Framework and REVLIMID REMS Program: A Methodology for REMS Assessment}

\author{
M. Chan-Liston ${ }^{1}$, J. Bourke ${ }^{1}$, P. Sheehan ${ }^{1}$, S. Niemcryk ${ }^{2}$, \\ M. Peng ${ }^{2}$, R. Bwire ${ }^{3}$, J. Freeman ${ }^{4}$, J. Backstrom ${ }^{5}$, R. Glasgow ${ }^{6}$ \\ ${ }^{1}$ U.S. REMS, Celgene Corporation, Summit, USA, ${ }^{2}$ Drug Safety, \\ Celgene Corporation, Summit, USA, ${ }^{3}$ Global Risk Management, \\ Celgene Corporation, Summit, USA, ${ }^{4}$ Global Drug Safety and Risk \\ Management, Celgene Corporation, Summit, USA, ${ }^{5}$ Office \\ of the CMO and Head of Global Regulatory Affairs, Celgene \\ Corporation, Summit, USA, ${ }^{6}$ Family Medicine, University \\ of Colorado School of Medicine, Denver, USA
}

Background: To standardize the assessment approach to Risk Evaluation and Mitigation Strategy (REMS) programs [1], FDA, academia, and professional societies have recommended alternative evaluation models be considered. Traditionally, REMS Assessment Reports are based on FDA determined criteria. The RE-AIM (Reach, Effectiveness, Adoption, Implementation, Maintenance) model [2], a public health program evaluation method, may be an applicable framework. In this study, we applied the RE-AIM framework to develop an alternative methodology for REVLIMID REMS ${ }^{\circledR}$ program assessment.

Objectives/Aim: To determine whether the RE-AIM framework could be applied successfully to the REVLIMID REMS program.

Methods: Using the International Society for Pharmacoepidemiology (ISPE) recommendations [3] and the RE-AIM framework, we evaluated all REVLIMID REMS elements (Implementation Plan and 4 Elements to Assure Safe Use (ETASU)). Data availability, applicability of metrics/measurement criteria to each RE-AIM domain, and the model's overall ability to determine if the REMS program is meeting its goals were considered.

Results: Three proactive, required REVLIMID REMS ETASU, applicable to each REMS stakeholder type (prescribers, pharmacies, patients) were selected for evaluation. The REACH domain included the percentage of patients enrolled based on the number requesting enrollment. EFFECTIVENESS metrics included mandatory REMS stakeholder tasks (e.g. prescriber and patient surveys, pharmacy dispense confirmation numbers, and pregnancy tests). The ADOPTION domain included certified prescribers and pharmacies who requested enrollment. IMPLEMENTATION metrics entailed the examination of enrollment processes, pharmacist REMS training compliance, and active REMS participation and compliance by enrolled stakeholders. For MAINTENANCE, stakeholder compliance with REMS tasks over time, reduction in
REMS burden (e.g. percentage of stakeholders using the fastest channel to perform mandatory REMS tasks), and REMS participation adherence metrics (e.g. percentage and times for flagged survey resolutions) were included.

Discussion: RE-AIM proved relevant to the REVLIMID REMS program, but the selection of appropriate REMS elements and the associated measurement criteria required multiple creative and innovative combinations of metrics. Challenges in applying RE-AIM include the potential for subjective selection of measurement tools from a myriad of data points, limited data availability, and determining the tools' relevance for accurate assessment of the REMS goals. However, after our methodology was determined, we felt that the RE-AIM framework was flexible and capable of adjustment to evaluate future changes in REMS program requirements.

\begin{tabular}{|c|c|c|}
\hline RE-AIM Domain & \begin{tabular}{|l|} 
Evaluated REMS \\
Element
\end{tabular} & Metrics/Measurement Criteria \\
\hline $\begin{array}{l}\text { REACH: } \\
\text { Extent to which patients who } \\
\text { requested enrollment } \\
\text { became enrolled }\end{array}$ & Patient ETASU & $\begin{array}{l}\text { Percentage of enrolled patients of those who requested } \\
\text { enrollment in the restricted distribution REMS }\end{array}$ \\
\hline $\begin{array}{l}\text { EFFECTIVENESS: } \\
\text { Percentage of stakeholders } \\
\text { demonstrating requisite } \\
\text { knowledge, behaviors, and } \\
\text { incidence of outcome }\end{array}$ & $\begin{array}{l}\text { Prescriber ETASU } \\
\text { Pharmacy ETASU } \\
\text { Patient ETASU }\end{array}$ & $\begin{array}{l}\text { Percentage of stakeholders demonstrating compliance with } \\
\text { and accuracy of initial responses for mandatory REMS tasks in } \\
\text { current reporting period: } \\
\text { - Prescriber survey } \\
\text { - REMS pharmacy confirmations-safe use verification } \\
\text { - Patient survey } \\
\text { - Pregnancy tests } \\
\text { - Pregnancy incidence proportion }\end{array}$ \\
\hline $\begin{array}{l}\text { ADOPTION: } \\
\text { Extent to which prescribers } \\
\text { and pharmacies that } \\
\text { requested enrollment } \\
\text { became enrolled/certified }\end{array}$ & $\begin{array}{l}\text { Prescriber ETASU } \\
\text { Pharmacy ETASU }\end{array}$ & $\begin{array}{l}\text { Percentage of enrolled/certified REMS stakeholders in the } \\
\text { restricted distribution REMS: } \\
\text { - Percentage of certified prescribers who requested } \\
\text { enrollment } \\
\text { - Percentage of certified pharmacies who requested } \\
\text { enrollment/inclusion in closed network }\end{array}$ \\
\hline $\begin{array}{l}\text { IMPLEMENTATION: } \\
\text { Percentage of stakeholders } \\
\text { implementing desired } \\
\text { behaviors, percentage of } \\
\text { trained stakeholders, and } \\
\text { barriers to implementation }\end{array}$ & $\begin{array}{l}\text { Prescriber ETASU } \\
\text { Pharmacy ETASU } \\
\text { Patient ETASU }\end{array}$ & $\begin{array}{l}\text { Percentage of enrolled/certified and participating REMS } \\
\text { stakeholders demonstrating REMS compliance: } \\
\text { - Active REMS participation by enrolled/certified } \\
\text { stakeholders } \\
\text { - REMS compliance by participating stakeholders } \\
\text { Percentage of implementation training and enrollment } \\
\text { measurements per active participants: } \\
\text { - REMS prescriber enrollment form receipts } \\
\text { - Training audit findings } \\
\text { - Annual certified Pharmacist/Counselor training rates } \\
\text { Appropriately completed Patient PPAFs }\end{array}$ \\
\hline $\begin{array}{l}\text { MAINTENANCE: } \\
\text { Extent to which the program } \\
\text { has been integrated at the } \\
\text { target sites into existing } \\
\text { workflow and if the program } \\
\text { is delivered as intended and } \\
\text { planned over time }\end{array}$ & $\begin{array}{l}\text { Prescriber ETASU } \\
\text { Pharmacy ETASU } \\
\text { Patient ETASU }\end{array}$ & $\begin{array}{l}\text { Percentage of stakeholders demonstrating compliance with } \\
\text { and accuracy of initial responses for mandatory REMS tasks, } \\
\text { over time: } \\
\text { - Prescriber survey } \\
\text { - REMS pharmacy confirmations-safe use verification } \\
\text { - Patient survey } \\
\text { - Pregnancy tests } \\
\text { - Pregnancy incidence proportion } \\
\text { Stakeholder administrative REMS burden reduction metrics: } \\
\text { - Prescriber surveys, pharmacy safe-use verifications } \\
\text { (Obtaining confirmation number), patient enrollments }\end{array}$ \\
\hline & & $\begin{array}{l}\text { and patient surveys performed by fastest channel (live } \\
\text { phone call, IVR, or on-line) } \\
\text { - Call center service level } \\
\text { REMS Participation adherence metrics (REMS sponsor's } \\
\text { speed of resolution of issues that leads to very low gap in } \\
\text { therapy and delay in treatment initiation): } \\
\text { - Percentage of mandatory prescriber surveys, pharmacy } \\
\text { dispense confirmation numbers obtained, and } \\
\text { mandatory patient surveys completed prior to pharmacy } \\
\text { dispense } \\
\text { - Gaps in therapy caused by REMS } \\
\text { - Delay in treatment initiation caused by REMS } \\
\text { - Resolution rate of flagged prescriber or patient surveys } \\
\text { Percentage of participating prescriber, pharmacy, and } \\
\text { patient complaints }\end{array}$ \\
\hline
\end{tabular}


Conclusions: We identified appropriate measurement criteria applicable to all 5 RE-AIM domains for the REVLIMID REMS program. Therefore, we believe that the RE-AIM framework could serve as a viable and quantifiable method for program assessment.

Further sources of information/References:

1. U.S. Food \& Drug Administration. For Industry. Background Materials for REMS Standardization and Evaluation Public Meeting: REMS Evaluation. Accessed at: http://www.fda.gov/ForIndustry/User Fees/PrescriptionDrugUserFee/ucm361888.htm on January 11, 2017.

2. Glasgow RE, Vogt TM, Boles SM. Evaluating the public health impact of health promotion interventions: the RE-AIM framework. APHA 1999; 89: 1322-7.

3. Benefit Risk Assessment Communication and Evaluation (BRACE) Special Interest Group. International Society for Pharmacoepidemiology. ISPE RESPONSE TO FDA QUESTIONS: Standardization and Evaluation of Risk Evaluation and Mitigation Strategies (REMS) Public Meeting on July 25-26, 2013. Accessed at http://www. pharmacoepi.org/pub/C62134BB-AD3F-1AFF-FABA25C83413971E on January 11, 2017.

\section{Antibiotics Use-Attitudes and Practices of First Year Nursing Students}

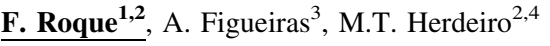 \\ ${ }^{1}$ Research Unit For Inland Development, Guarda Polytechnic, \\ Guarda, Portugal, ${ }^{2}$ Institute for Biomedicine, Medical Sciences \\ Department, University of Aveiro, Portugal, ${ }^{3}$ Consortium \\ for Biomedical Research in Epidemiology and Public Health, \\ Santiago de Compostela, Spain, ${ }^{4}$ Institute of Research and Advanced \\ Training in Health Sciences and Technologies, Gandra, Portugal
}

Introduction: Antibiotics misuse is an important cause of bacterial resistances which is associated to an increased health-treatment costs and arise in new adverse drug reactions [1-4]. In the last Eurobarometer published in 2016, about $16 \%$ of the Portuguese choose the nurses as trustworthy sources of information about antibiotics and resistances [5]. Nursing schools should educate their students with knowledge and awareness about this important issue. To design a tailored education, it is necessary to understand the practices and attitudes of students.

Aim: Identify attitudes and practices of first year nursing students about antibiotics consumption.

Methods: We conducted a cross-sectional study. A self-administered questionnaire on attitudes and practices related to antibiotic use was applied to 79 nursing students from the first year of the course.

Results: From the 79 participants, two never had taken antibiotics and other two did not know if they have already taken antibiotics anytime. About $50 \%$ have taken antibiotics in the previous 12 months, and about $20 \%$ have taken antibiotics without medical prescription (33\% of them in the previous 12 months). The students that have taken antibiotics without previous medical prescription said that they obtained it in the pharmacy $(67 \%)$ or have used the leftovers from previous treatments $(33 \%)$. The main reason for take antibiotics without medical prescription is because they know which normally does them good. The main situations for antibiotic use (without prescription) were throat pain (80\%), colds $(47 \%)$, fever (27\%) and toothache (27\%). When asked about what they usually do with the leftover tablets, about $47 \%$ said that they kept them to take it in other situations, and, $28 \%$ answer that they put the tablets in the household waste. Only $21 \%$ said that they return medicines to the pharmacy. About $24 \%$ of all the participants in the study consider that it is not necessary go to the doctor to treat clinical situations that seems similar to clinical situations that have occurred previously.

Conclusions: Students need educational interventions that change their attitudes and their behaviours related to antibiotic use.

Further sources of information/References:

1. Cosgrowe SE, Carmeli Y.The impact of antimicrobial resistance on health and economic outcomes. Clin Infect Dis 2003;36:1433-1437. 5

2. Howard DH,Scott RD. The economic burden of drug resistance. Clin Infect Dis 2005;41:S283-S286

3. ECDC/EMEA Joint Working Group. Technical Report-The Bacterial Challenge: Time to React. Stockholm, Sweden (2009). http://ecdc.europa.eu/en/publications/

4. Bell BG, Schellevis F, Stobberingh E, Goossens H, Pringle M. A systematic review and meta-analysis of the effects of antibiotic consumption on antibiotic resistance. BMC Infect. Dis 2014; 14(:13

5. European Commission: Special Eurobarometer 445. Antimicrobial Resistance (in the EU). 2016, Available online http://ec.europa.eu/dgs/ health_food-safety/amr/docs/eb445_amr_generalreport_en.pdf

\section{Rapid onset of very high INR values when roxithromycin prescribed with warfarin challenges the accepted mechanism}

R. Savage ${ }^{1,2,3}, \underline{\text { M. Tatley }}{ }^{1}$

${ }^{I}$ New Zealand Pharmacovigilance Centre, University Of Otago, Dunedin, New Zealand, ${ }^{2}$ Dept General Practice, University of Otago Christchurch, Christchurch, New Zealand, ${ }^{3}$ Uppsala Monitoring Centre, Uppsala, Sweden

Introduction: Recently two reports to the New Zealand Centre for Adverse Reactions Monitoring (CARM) highlighted very high International Normalised Ratio (INR) values observed in patients as early as day 3 or 4 after roxithromycin was added to long term warfarin treatment. The interaction was first observed in New Zealand and Australia and published in 1995 [1]. Macrolide antibiotics are known to interact with warfarin. CYP 3A4 inhibition is the postulated mechanism although only the less active R-enantiomer of warfarin is a substrate. Roxithromycin is a weak CYP 3A4 inhibitor. It is considered less likely than erythromycin to interact with warfarin to a clinically important extent which conflicts with the observed early marked effect on prothrombin activity. All the reports of this interaction in the CARM database and relevant publications were therefore reviewed.

Aim: To investigate the potentiating effect of roxithromycin on warfarin activity as described in adverse reaction reports to CARM.

Results: Thirty CARM reports of increased INR values were identified, nine with haemorrhage. The median age was 66.8 years. A feature of the case series was the rapid onset of very high INR values Seven patients had values measured on day 3 of roxithromycin treatment and these ranged from 4.3 to 16.7 (median 8.8). A high proportion of interactions was observed between warfarin and roxithromycin compared with other macrolides and compared with other roxithromycin interactions also considered due to CYP 3A4 inhibition suggesting that this is not the mechanism for the warfarin interaction. This observation closely mirrored Australian experience [2].

Discussion: Pharmacokinetic studies did not show an effect of roxithromycin on CYP1A2-dependent $(R)$-warfarin 7-hydroxylation or CYP2C9-dependent ( $S$ )-warfarin 7-hydroxylation, but confirmed that it is a weak CYP 3A4 inhibitor [3]. Older age and polypharmacy have been 
suggested as contributors to the interaction in two case series $[1,4]$. When increased INR values are observed with broad spectrum antibiotics and warfarin and there is no obvious pharmacokinetic mechanism, reduced bacterial synthesis of vitamin $\mathrm{K}$ is the proposed likely cause. Is there likely to be a more pronounced effect in patients with VKORC1 variants? Would the effect on bacteria be in keeping with the rapid onset of high INR values?

Conclusion: The INR increases observed in the CARM reports suggests that even advice to measure the INR on day 3 of roxithromycin treatment [5] may not be early enough for some patients. Further avoidance of harm may be possible with greater understanding of the mechanism.

Further sources of information/References:

1. Ghose K, Ashton J, Rohan A. Possible interaction of roxithromycin with warfarin. Updated review of ADR reports. Clin Drug Invest 1995; 10: 302-9

2. ADRAC. Interactions with macrolides. Adverse Drug React Bull 2006; 25

3. Yamazaki H, Shimada T. Comparative studies of in vitro inhibition of cytochrome P450 3A4-dependent testosterone 6b-hydroxylation by roxithromycin and its metabolites, trolandeomycin and erythromycin. Drug Metab Dispos 1998; 26: 1053-1057

4. Rotella J-A, Taylor DM. Concomitant roxithromycin use amongst elderly patients taking warfarin resulting in significant over-anticoagulation. J Pharm Pract Res 2015; 45: 182-185

5. Medsafe editorial team. Check INR after starting roxithromycin for patients on warfarin. Prescriber Update 2015; 36: 3 http://www. medsafe.govt.nz/profs/PUArticles/March2015Roxithromycin.htm

\section{How do Adverse Drug Reactions Influence the Patient's Daily Life? Qualitative Analysis on Spontaneous Reports by Patients}

\author{
L. Rolfes ${ }^{1,2}$, M. Haaksman ${ }^{3}$, F. van Hunsel ${ }^{1,2}$, \\ E. van Puijenbroek ${ }^{1,2}$
}

${ }^{1}$ Pharmacovigilance Centre Lareb, Den Bosch, the Netherlands, ${ }^{2}$ University of Groningen, Groningen Research Institute of Pharmacy, Unit of PharmacoTherapy, Epidemiology \& -Economics, Groningen, the Netherlands, ${ }^{3}$ University of Applied Sciences Utrecht, Institute of Pharmaceutical Business Administration, Utrecht, the Netherlands

Background: Patients bring a new dimension to pharmacovigilance by reporting frequently about the impact of adverse drug reactions (ADRs) on their daily life. However, little is known about what aspects of the patient's daily life are being influenced due to ADRs.

Objective: To analyse how ADRs, reported to a pharmacovigilance centre, influence the patient's daily life.

Methods: A qualitatively analysis on information about the impact of ADRs on the patient's daily life. Since March 2017, the Netherlands pharmacovigilance centre Lareb added a question about the impact of the ADR, asked for each reported ADR, to the patient reporting form. The answer option was a 5-point scale ranging from 'not at all' to 'very much', followed by an open text field in which patients could explain their given answer in their own words.

All patient reports from 6 to 29 March 2017 were selected from the Lareb database. Only those in which the patient reported for themselves, and the open text field was filled, were included for analysis. All open text fields were coded individually by two researchers (LR, MH), using content analysis. Differences were discussed until consensus was reached. Prior to the analysis a coding scheme was drafted. This was adapted during this interactive coding process.

Results: In total 194 reports were selected, including 236 drug-ADR associations. Content analyses indicated that ADRs influenced the patient's daily life on several aspects. Table 1 summarizes the five items most mentioned.

The severity of the ADR was most often mentioned. This e.g. included that the ADR was distracting, annoying or painful. But there were also patients that considered the ADR to have little or no impact, e.g. when they were treated for it. Interestingly, for the impact on mood or concentration, over half of the reactions was about anxiety because of possible consequences of the ADR. For physical impartment, reactions were mainly general descriptions, e.g. fatigue or problems with walking. For limitations in social activities, it was often specifically mentioned that the ADR influenced their work or family-life.

Conclusion: Several aspects causing ADRs to influence the patient's daily life were indicated. Patients took the opportunity to explain in their own words how ADRs influenced their daily life. This is valuable to understand the actual impact of ADRs. Moreover, it enables healthcare professionals to provide dedicated information on the consequences of these ADRs from a patient's perspective.

Table 1. Five most mentioned items of how ADRs influence the patient's daily life
\begin{tabular}{|l|l|}
\hline Item & Number (\%) \\
\hline Severity of the adverse drug reaction & $153(40 \%)$ \\
\hline Impact on mood or concentration & $56(15 \%)$ \\
\hline Overall (change in) health & $50(15 \%)$ \\
\hline Physical impairment & $39(10 \%)$ \\
\hline Limitations in social activities & $34(9 \%)$ \\
\hline
\end{tabular}

\section{Suspected adverse reactions to cannabis galenic preparations for medical use in Italy}

\author{
F. Menniti-Ippolito ${ }^{1}$, R. Da Cas ${ }^{1}$, E. Gallo ${ }^{2}$, F. Firenzuoli ${ }^{3}$
}

${ }^{1}$ Italian National Institute Of Health, Rome, Italy, ${ }^{2}$ Department of Experimental and Clinical Medicine, University of Florence, Florence, Italy, ${ }^{3}$ Center for Integrative Medicine, Careggi University Hospital, University of Florence, Florence, Italy

Introduction: The interest of the scientific community and of patients for medical use of cannabis is currently very strong. Only one drug containing cannabis was registered for second line treatment in moderate to severe spasticity due to multiple sclerosis (MS). However, some evidence in the literature suggests cannabis use for neuropathic pain, pain in cancer patients, nausea and vomiting induced by chemotherapy and fibromyalgia $[1,2,3]$.

Aim: To describe suspected adverse reactions (ARs) associated with cannabis galenic preparations for medical use.

Methods: Spontaneous reports of suspected ARs to natural health products (including galenic preparations containing herbals) are collected in Italy within the Italian Surveillance System of suspected adverse reactions to Natural Health Products coordinated by the National Institute of Health, in collaboration with the Italian Medicines Agency and the Ministry of Health

Results: At December 2016 twenty-six suspected adverse reactions to cannabis for medical use were reported. Median age of patients was 56 years (range 22-80), women represented 69\% of reports. Six reports were related to serious reactions. Cannabis was used mainly for neuropathic pain, in two cases cannabis was assumed as palliative care, in one case the 
reason for use was lack of appetite and cancer pain and in two cases headache. In 15 cases concomitant use of drugs was reported.

Psychiatric reactions were reported (dysphoria, panic attack, visual hallucinations, stupor, drowsiness, major depression...); lack of efficacy; dermatological symptoms (itchiness, redness and swallowing of eyelids and face); laryngospasm. Causality assessment was performed for all reactions and the association between cannabis use and the reaction was always probable. Cannabis was assumed almost in all cases but one by decoction. In one case cannabis was extracted in oil.

Conclusion: Cannabis for medicinal use is usually well tolerated. The adverse effects collected are mainly due to dosage errors, mode of administration or individual differences in THC absorption and metabolism. However, it is important to monitor cannabis medical use in the population and to continually evaluate its safety.

\section{Further sources of information/References}

1. Haney M, et al. Dronabinol and marijuana in HIV-positive marijuana smokers. Caloric intake, mood, and sleep. J Acquir Immune Defic Syndr 2007; 45: 545-54

2. Koppel BS, et al. Systematic review: efficacy and safety of medical marijuana in selected neurologic disorders: report of the Guideline Development Subcommittee of the American Academy of Neurology. Neurology 2014; 82: 1556-63

3. Smith LA, et al. Cannabinoids for nausea and vomiting in adults with cancer receiving chemotherapy. Cochrane Database Syst Rev 2015; 12: CD009464

\section{Tailoring signal detection methodologies in a global database to focus on safety concerns reported by patients}

R. Chandler ${ }^{1}$, S. Watson ${ }^{1}$, B. Grundmark ${ }^{1}$, L. Härmark ${ }^{2}$, K. Star ${ }^{1}$, H. Taavola ${ }^{1}$, F. van Hunsel ${ }^{2}$

${ }^{1}$ Uppsala Monitoring Centre-WHO Collaborating Centre

For International Drug Monitoring, Uppsala, Sweden, ${ }^{2}$ Netherlands Pharmacovigilance Centre Lareb, 's-Hertogenbosch, the Netherlands

Introduction: A recent systematic review summarized the current evidence on the value of patient reporting into pharmacovigilance systems. [1] There is literature to suggest that patient reports contribute to signal detection [2-3]. However, descriptions of methodologies for using patient reports in signal detection are scarce, and published experiences of how patient reports are used in national pharmacovigilance centers are limited to a few countries [4-6].

Aim: To explore the contribution of patient reports to signal detection in an international database of suspected adverse drug reactions (ADRs).

Methods: Data was retrieved from the WHO global database of individual case safety reports (ICSRs), VigiBase, in September 2016. Suspected duplicate reports and reports from studies were excluded. Drug-ADR combinations were generated and restricted to report series with at least $50 \%$ patient ICSRs. Further, only combinations with at least one report received after 2014 , from $\geq 2$ countries, and with $\leq 30$ patient ICSRs were included. vigiRank, an algorithm using multiple-strength-of-evidence aspects, was used to prioritize combinations for assessment. In the assessment of each combination, the product information for health care providers as well as patient information leaflets were reviewed for information on ADRs.

Results: A total of 212 combinations were assessed; 20 (9\%) combinations resulted in 8 signals communicated within the WHO program for international drug monitoring. Five signals described new, unlabeled suspected ADRs; 3 described new aspects of known ADRs. An example of the former is the signal of panic attacks with levothyroxine; an example of the latter is the signal of severe genital pruritus leading to non-compliance with SGLT-2 inhibitors. Reviews of patient information leaflets were performed; examples of poor consistency with product information for physicians were found, such as placement of ADR terms and descriptions of ADRs. Patient narratives were confirmed to provide details regarding the experience of the ADR and its impact on the quality of life of the reporter; furthermore, there is evidence in narratives that patients make causality and benefit/harm assessments themselves.

Conclusions: Safety concerns described in patient reports can be identified in a global database including previously unknown ADRs as well as new aspects of known ADRs. Patient reports provide unique information valuable in signal assessment, and they should be included in signal detection as far as is possible. Novel methodologies to highlight patient reports in statistical signal detection can further improve the contribution of patient reports to pharmacovigilance.

\section{Further sources of information/References:}

1. Inácio $\mathrm{P}$, Cavaco $\mathrm{A}$, Airaksinen $\mathrm{M}$. The value of patient reporting to the pharmacovigilance system: a systematic review. $\mathrm{Br} \mathrm{J}$ Clin Pharmacol 2017; 83: 227-46

2. van Hunsel F, Talsma A, van Puijenbroek E, de Jong-van den Berg L, van Grootheest K. The proportion of patient reports of suspected ADRs to signal detection in the Netherlands: case-control study. Pharmacoepidemiol Drug Saf 2011r;20(3):286-91

3. Hazell L, Cornelius V, Hannaford P, Shakir S, Avery AJ; Yellow Card Study Collaboration. How do patients contribute to signal detection? : A retrospective analysis of spontaneous reporting of adverse drug reactions in the UK's Yellow Card Scheme. Drug Saf 2013;36(3):199206

4. de Langen J, van Hunsel F, Passier A, de Jong-van der Berg L, van Grootheest K. Adverse drug reaction reporting by patients in the Netherlands: three years of experience. Drug Saf 2008; 31:515-24

5. Aagaard L, Nielsen LH, Hansen EH. Consumer reporting of adverse drug reactions: a retrospective analysis of the Danish adverse drug reaction database from 2004 to 2006. Drug Saf 2009; 32: 1067-74

6. Avery AJ, Anderson C, Bond CM, Fortnum H, Gifford A, Hannaford $\mathrm{PC}$ et al. Evaluation of patient reporting of adverse drug reactions to the UK "Yellow Card Scheme": literature review, descriptive and qualitative analyses, and questionnaire surveys. Health Technol Assess 2011; 15: 11-234

\section{Adverse Drug Reactions Notifications by Patients}

\author{
M. T. Herdeiro ${ }^{1,2}$, P.C. Mastroianni ${ }^{3}$, F.R. Varallo ${ }^{3,4}$, F. Roque $^{\mathbf{1 , 5}}$
}

${ }^{1}$ Institute for Biomedicine, Medical Sciences Department, University of Aveiro, Aveiro, Portugal, ${ }^{2}$ Institute of Research and Advanced Training in Health Sciences and Technologies, Gandra, Postugal, ${ }^{3}$ São Paulo State University, School of Pharmaceutical Sciences, Araraquara, Brazil, ${ }^{4}$ Americo Brasiliense State Hospital, Araraquara, Brazil, ${ }^{5}$ Research Unit for Inland Development, Guarda Polytechnic, Guarda, Portugal

Introduction: Adverse drug reactions (ADRs) are an important public problem in terms of patient morbidity, mortality and costs for health systems. Therefore, reporting of suspected ADRs is fundamental to drug safety surveillance. Substantial underreporting exists and is the system's 
main limitation. In Portugal, the patients began to report ADR in July 2012, with the implementation of Directive 2010/84/EU.

Aim: Analyse the number of ADR notifications by patients (users) during the last 5 years.

Methods: We conducted an observational study based on the annual reports published on the National Authority of Medicines and Health Products, I.P (INFARMED) website, about notifications and cases of spontaneous ADR received by National Pharmacovigilance System (NPS) from 2012 through 2016. The notification of spontaneous reports data could include duplicates not yet detected.

Results: The notification of ADR by patients (users) started in July 2012 . In 2012, from the total $(n=3104)$ of notifications received in NPS, 1411 were by healthcare professionals (HCPs), 16 were by patients and 1677 by pharmaceutical industry. In 2013, the number of notifications by patients increase to 50 notifications, the total of notifications by direct via (from HCPs and patients) was 1526, the total of notifications including the indirect via (from pharmaceutical industry) was 3461 . The NPS received in 2014, a total of 4618 notifications, from patients were 175 ADR notifications of which 21 were online, through the ADR portal, an increase of $4 \%$ relative to 2013. In 2015, the patient's ADR reports increase to 215 (19 via online) and the total number of notifications was 5690. In the last year, 2016, the number of ADR notifications received are similar to 2015 (total of 5698). From direct via there are 2786 notifications, from which, $329(11 \%)$ are from patients.

Conclusions: Accordingly to data published in the INFARMED website, the number of notifications by patients has increased from 2012 to 2016 . However, this number is lower compared with other countries. The reporting from patients is very important to complement the reporting from HCPs.

Acknowledgements: Authors wish to acknowledge to Institute for Biomedicine, University of Aveiro (iBiMED/UA) and to Research Unit for Inland Development, Polytechnic of Guarda (UDI/IPG), that support this work.

Further sources of information/References:

1. The European Parliament and the Council of the European Directive 2010/84/EU of the European Parliament and of the Council of 15 December 2010 amending, as regards pharmacovigilance, Directive 2001/83/EC on the community code relating to medicinal products for human use. Official J Eur Commun 348: 74-99.

2. Ministry of Health. DecreeLaw 20/2013, of 14 de Februry 2013, amending DecreeLaw 176/2006 of 30 August 2006. Diário da República.

3. Banovac M, Candore G, Slattery J, Houÿez F, Haerry D, Genov G, Arlett P. Patient Reporting in the EU: Analysis of EudraVigilance Data. Drug Saf. 2017 Jul;40(7):629-645.

\section{Potentially inappropriate medicines in elderly patients-studies using the European screening tool, EU(7)-PIM list}

\author{
F. Roque $^{\mathbf{1 , 2}}$, P.C. Mastroianni ${ }^{3}$, F.R. Varallo ${ }^{3,4}$, M.T. Herdeiro ${ }^{1,5}$ \\ ${ }^{1}$ Institute for Biomedicine, Medical Sciences Department, University \\ of Aveiro, Aveiro, Portugal, ${ }^{2}$ Research Unit for Inland Development, \\ Guarda Polytechnic, Guarda, Portugal, ${ }^{3}$ São Paulo State University, \\ School of Pharmaceutical Sciences, Araraquara, Brazil, ${ }^{4}$ Americo \\ Brasiliense State Hospital, Araraquara, Brazil, ${ }^{5}$ Institute of Research \\ and Advanced Training in Health Sciences and Technologies, \\ Gandra, Portugal
}

Introduction: In Europe, 24 per cent of the population is already aged 60 years or over and that proportion is projected to reach 34 per cent in 2050 [1]. The presence of multiple diseases affects more than half of the elderly population, with increased prevalence in the older population, making them a polimedicated population. Polypharmacy and modified pharmacokinetics and/or pharmacodynamics drug characteristics make older people more prone to adverse drug events, frequently associated with potentially inappropriate medication (PIM) use. Recently, it was published a list of potentially inappropriate medications for older people, consented by experts from seven European countries, the EU(7)-PIM list [2].

Aim: To review studies that analyzed potentially inappropriate medicines in elderly patients according to the EU (7)-PIM list.

Methods: We conducted a search on MEDLINE PubMed database and reviewed the relevant literature on potentially inappropriate medicines in elderly patients. For inclusion in the revision, studies should measure the prevalence of PIMs according to the EU(7)-PIM list.

Results: The search retrieved 6 studies and, one of the studies is the publication of the EU (7)-PIM list, and one study analyses the availability of these medicines in the Belgian market. Four studies were included in the review analysis. From these, 3 studies were developed in Europe and the other one was developed in Brazil. Two studies used two criteria for evaluation of PIMs: the EU (7)-PIM list and the STOPP criteria, in Croatiás study, and, the EU (7)-PIM list and Beers Criteria in the Lithuaniás study. The Brazilian study used 4 criteria to evaluate the prevalence of PIMs, the EU (7)-PIM list, the STOPP criteria, the Beers criteria, and the Taiwan criteria. High prevalence of PIMs were found in all studies, and for all criteria used. The other European study evaluate PIMs just by EU (7)-PIM list. The PIMs evaluated by STOPP criteria varied between 46.2 and $69 \%$, by the Beers criteria varied between 24 and $50 \%$, and for the EU (7)-PIM list, values ranged between 40.9 and $66,7 \%$. In two studies EU(7)-PIM list identified more PIMs then the other criteria used (STOPP and Beers) and in one study STOPP criteria identified more PIMs than EU (7)-PIM list.

Conclusions: There are few published studies using the EU(7)-PIM list, a screening tool developed to allow the identification and comparison of PIMs prescription across European countries. It is important to develop more studies and evaluate the feasibility for each European country.

Acknowledgements: iBiMED/UA; UDI/IPG.

Further sources of information/References

1. United Nations (2015). World Population Prospects. Key findings and Advance tables: The 2015 Revision. https://esa.un.org/unpd/wpp/ publications/files/key_findings_wpp_2015.pdf

2. Renom-Guiteras A, Meyer G, Thürmann PA. The EU(7)-PIM list: a list of potentially inappropriate medications for older people consented by experts from seven European countries. Eur J Clin Pharmacol 2015;71:861-75

\section{Fetal Impregnation with Olanzapine: A Case Report}

\author{
A. Viard ${ }^{1}$, M. Damin-Pernik ${ }^{1}$, S. Rapin ${ }^{2}$, M.N. Beyens ${ }^{1}$
}

${ }^{1}$ Pharmacovigilance Center, CHU Saint-Etienne, Saint Priest En Jarez, France, ${ }^{2}$ Department of Neonatal Intensive Care, CHU SaintEtienne, Saint Priest en Jarez, France

Introduction: A case of fetal olanzapine impregnation was notified in March 2017 in our pharmacovigilance center. Olanzapine is an antipsychotic agent, which can be used during pregnancy only if the potential benefit justifies the potential risk to the fetus (neonatal withdrawal symptoms if treatment is continued until delivery with a greater frequency 
of neonatal intensive care admission (30.8\%)) [1]. The long elimination half-life of olanzapine $(35 \mathrm{~h})$ is a risk factor of accumulation in the newborn.

Methods: To describe a case report and cases of French Pharmacovigilance Database

Results: A 31-year-old woman is treated by haloperidol (50 mg/week) and olanzapine $(15 \mathrm{mg} /$ day) for schizophrenia, during her pregnancy. At $28 \mathrm{WA}+4 \mathrm{~d}$ haloperidol was stopped and olanzapine dosage was increased at $20 \mathrm{mg} /$ day. The mother developed gestational diabetes (insulin + therapeutic education) from the 5th month of pregnancy. She was admitted on March 21, 2017 for a caesarean delivery (38WA+4d) owing to an abnormal fetal heart rhythm.

The newborn was a boy (birth weight $4635 \mathrm{~g}$, height $52.5 \mathrm{~cm}$ ) and has an Apgar score of 6/7/10/10. He presented a hypoglycemia persistent up to D3, respiratory distress, and a progressive axial hypotonia which motive his transfer to neonatal intensive care unit. Then, he was intubated from D1 to D4 and presented comatose state with tremor. The hypothesis of fetal impregnation was evocated at $\mathrm{H} 8$ and dosage of olanzapine confirmed this diagnosis: $35 \mu \mathrm{g} / \mathrm{ml}$ (H8), $13 \mu \mathrm{g} / \mathrm{ml}$ (H40), $10 \mu \mathrm{g} / \mathrm{ml}$ (H64) [Therapeutic rate in adults: $20-80 \mu \mathrm{g} / \mathrm{ml}$ ]. The research of other toxics remained negative (benzodiazepine, barbiturates and antidepressants). $\mathrm{He}$ left hospital without neurological damage at 16 days of life.

Discussion: In the French Pharmacovigilance Database, 68 cases of pregnancy exposed to olanzapine are notified of which 22 cases could correspond to a syndrome of fetal impregnation. Olanzapine is the only suspected drug for only 2 cases. Among these 22 cases, we find most often respiratory distress resolving in $24 \mathrm{~h}$, hypotonia and tremors, they selfresolve in a few days. Problems of suction or feeding, resolved more slowly (persisting disorders of suction for 13 days in one case). Tremor and eating disorders with delayed onset may also correspond to withdrawal symptoms linked to the long half-life. Finally, we find a case of hypoglycemia, without any other symptom associated, normalized at D4. Conclusion: This case is the first French documented case (with concentration of toxic) of impregnation of a new born with only olanzapine, associated with hypoglycemia.

Further sources of information/References:

1. Newport DJ, Calamaras MR, DeVane CL, et al: Atypical antipsychotic administration during late pregnancy: placental passage and obstetrical outcomes. Am J Psychiatry 2007; 164(8):1214-20

\section{Enhanced Safety Surveillance for Seasonal Influenza Vaccines: A Year-to-Year Comparison of Suspected Adverse Drug Reactions}

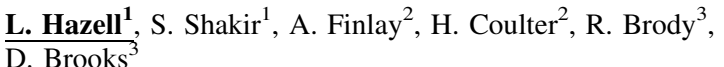

${ }^{1}$ DSRU Education \& Research Ltd., Southampton, UK, ${ }^{2}$ AstraZeneca, Luton, UK, ${ }^{3}$ AstraZeneca, Gaithersburg, USA

Background: In 2014, the European Medicines Agency issued an interim guidance for enhanced safety surveillance (ESS) on seasonal influenza vaccines [1]. In response, we designed and implemented a passive ESS programme to monitor the safety of an intranasal flu vaccine used in children aged 2 to 17 years (Fluenz ${ }^{\circledR}$ Tetra) [2].

Objective: To measure the frequencies of suspected adverse drug reactions (sADRs) in children receiving Fluenz ${ }^{\circledR}$ Tetra during the early 2015 and 2016 influenza seasons in England.
Methods: The ESS programme was approved in each season by an NHS Research Ethics Committee. School immunisation providers and general practices across England were invited to take part with support of the UK Clinical Research Network. Vaccinees or parents/guardians received a Safety Report Card (SRC) after vaccination with Fluenz ${ }^{\circledR}$ Tetra and were asked to return this to the vaccine manufacturer if children experienced sADRs. Reporting rates for sADRs were calculated using concurrent denominator data provided by immunisation teams for each MedDRA preferred term (Version 19.1) [3].

Results: The ESS programme captured data for approximately 8 weeks (October/November) in each season. The number of SRCs issued increased from 8753 (67 sites) in 2015 to 12,610 (91 sites) in 2016. The overall sADR reporting rate was lower in the 2016 season (112 SRCs returned; 8.9 per 1000 SRCs issued) compared to that in 2015 (165 returned; 18.9 per 1000 SRCs issued). The majority of sADRs were for children aged 2 to 4 years $(62.0 \%$ in 2015 and $59.4 \%$ in 2016). The most frequently reported sADRs were similar in both seasons (Table 1). One serious sADR was reported in the 2015 ESS programme (due to hospitalisation) while no serious ADRs were reported in the 2016 season.

Discussion: The ESS approach has limited power to detect signals of rare events but may have utility in understanding variation in reporting of more frequently occurring reactogenic events from season to season. Although the absolute number of sADRs reported was low, the reporting rate in the ESS programme was considerably higher than the overall UK reporting rate, considering SADRs from the vaccinated population for the same time period.

Conclusion: We found no evidence of any increased frequencies of minor expected events in 2016 relative to 2015 or other safety signals. The ESS allows collection of near real-time patient reported outcomes following vaccination and corresponding denominator data and can be considered an important extension to routine pharmacovigilance activities.

Table 1 - Reporting Rate of Suspected ADRs in 2015 and 2016 Fluenz Passive Enhance Safety Surveillance Programme.

\begin{tabular}{|l|l|l|}
\hline \multirow{2}{*}{ sADRs reported } & \multicolumn{2}{|l|}{ No. of sADRs per 1000 SRCs issued (95\% CI) } \\
\cline { 2 - 3 } & 2015 & 2016 \\
\hline Overall & $18.9(16.1-21.9)$ & $8.9(7.3-10.7)$ \\
\hline Most Frequent sADRs: & $6.3(4.7-8.2)$ & $2.4(1.6-3.4)$ \\
\hline Rhinorrhoea & $3.5(2.4-5.0)$ & $1.8(1.2-2.7)$ \\
\hline Pyrexia & $4.0(2.8-5.6)$ & $1.5(0.9-2.3)$ \\
\hline Cough & $2.6(1.7-3.9)$ & $1.6(0.9-2.4)$ \\
\hline Headache & \multicolumn{2}{l}{} \\
SADR = suspected adverse drug reaction
\end{tabular}

Further sources of information/References:

1. European Medicines Agency (EMA). Interim guidance on enhanced safety surveillance for seasonal influenza vaccines in the EU. Published: 10/4/2014. Available at URL: http://www.ema.europa.eu/ docs/en_GB/document_library/Scientific_guideline/2014/04/WC5001 65492.pdf Accessed 31 ${ }^{\text {st }}$ March 2017

2. MedImmune UK Ltd. Summary of Product Characteristics-Fluenz Tetra $^{\circledR}$. Last updated 29/2/2016. Available at URL: http://www. ema.europa.eu/docs/en_GB/document_library/EPAR_-_Product_Infor mation/human/002617/WC500158412.pdf. Accessed 24 ${ }^{\text {th }}$ June 2016

3. Medical Dictionary for Regulatory Activities. Maintenance and Support Services

4. Organisation Available at URL: http://www.meddra.org/ Accessed $31^{\text {st }}$ March 2017 
73 Adherence to therapeutic in outpatients: Literature Review

\author{
M.I.B. Ribeiro ${ }^{1,2,3}$, L.M.F. Nascimento ${ }^{4}$, M.T. Herdeiro ${ }^{5,6}$ \\ F. Roque ${ }^{1,5,7}$
}

${ }^{1}$ Health Sciences School, Polytechnic of Guarda, Guarda, Portugal, ${ }^{2}$ Department of Exact and Social Sciencies, Agriculture School, Institute Polytechnic of Bragança, Bragança, Portugal, ${ }^{3}$ Centre of Transdisciplinary Development Studies, Vila Real, Portugal, ${ }^{4}$ Department of Diagnostic and Therapeutic Technologies, Health School, Institute Polytechnic of Bragança, Bragança, Portugal, ${ }^{5}$ Institute for Biomedicine, Medical Sciences Department, University of Aveiro, Aveiro, Portugal, ${ }^{6}$ Institute of Research and Advanced Training in Health Sciences and Technologies, Gandra, Portugal, ${ }^{7}$ Research Unit For Inland Development, Guarda Polytechnic, Guarda, Portugal

Introduction: Non-adherence to therapeutic is a widespread problem that contributes to the worsening of chronic diseases and to increase the expenditure on health care [1-3].

Objective: To present a review of the literature on the subject "Adherence to therapy" in outpatients.

Methods: A search was carried out in the Medline database and the combinations of words Medication, Adherence and Outpatients were used for the period from 2010 to 2017. including only articles that adopted the Morisky Medication Adherence Scale (MMAS) to measure adherence to medication and studies developed in hospital context. In addition, articles with non-conventional therapies were excluded, namely those that resorted to the use of medicinal plants. Information was collected on the place of the study, period, type of study, sample, pathology, adherence to therapy in terms of rate or score (mean) and factors identified as being associated with non-adherence to therapy.

Results: The search retrieved 461 publications and 44 were included in the analysis. The included studies are from Europe, Asia, Africa and America. Most of the studies were observational (84.1\%). The remaining studies were interventional studies $(15.9 \%)$. There were, also, 4 multicenter studies. In most studies, the Morisky scale was used with 8 item and only $31.8 \%$ used the shorter scale with 4 items. The sample sizes ranged from 34 to 2595 chronic patients and were mostly non-probabilistic (convenience) $(75 \%)$. The most common pathologies were: diabetes, hypertension, diabetic retinopathy and hypercholesterolemia (34.1\%); psychiatric diseases (25\%), cardiovascular diseases (18.2\%), among others. Taking into account the variable "adherence to therapeutic", $84.5 \%$ of the studies used a dichotomous variable. The others determined the score (mean with associated standard deviation). In general, therapeutic adherence was low. Considering the adherence rates, the lowest were recorded in the treatment of hypercholesterolemia (7.4\%) and the highest was recorded in the treatment of hypertension in patients with apnea (83\%). It should be noted that the cut-off point did not remain the same in all studies. The factors most commonly referred to as being associated with non-adherence were age, forgetfulness, lack of social support, the complexity of intake and stopping medication when the patient feels better.

Conclusion: Interventional studies show significant improvements in adherence, comparing the situation before and after the intervention. Therefore, it is a priority to implement measures that improve adherence to therapy. Acknowledgements: This work is supported by FEDER/COMPETE 2020 [Project No. 006971 (UID/SOC/04011); Funding Reference: POCI-010145-FEDER-006971]; and by UDI/IPG and iBiMED/UA.

Further sources of information/References

1. Conn VS, Ruppar TM, Chan KC, Dunbar-Jacob J, Pepper GA, Geest SD. Packaging interventions to increase medication adherence: systematic review and meta-analysis. Curr Med Res Opin 2015, 31:145-60

2. Ababneh MA, Al-Azzam SI, Alzoubi KH, Rababa'h AM. Adherence in outpatients taking warfarin and its effect on anticoagulation control in Jordan. Int J Clin Pharm 2016, 38:816-21

3. Napolitano F, Napolitano P, Angelillo IF. Collaborative Working Group. Medication adherence among patients with chronic conditions in Italy. Eur J Public Health 2016 26:48-52

\section{Beyond co-occurrence-based ADR detection from Social Media}

\author{
D. Bollegala ${ }^{1}$, S. Maskell ${ }^{1}$, M. Pirmohamed ${ }^{1}$
}

${ }^{1}$ University of Liverpool, Liverpool, $\underline{U K}$

Introduction: Detecting adverse drug reactions (ADRs) is an important task that has direct implications for the use of that drug for patients, pharmaceutical companies, health organisations and regulators. A promising approach for detecting ADRs is to use social media. A high level of correlation between a drug name and an ADR can be regarded as an indication of a potential ADR associated with that drug. Although numerous association measures have been proposed in the signal detection community for identifying ADRs, these measures tend to ignore causality. Aim: Our aim was to develop a causality measure that can detect an adverse reaction caused by a drug rather than merely being a correlated signal.

Methods: We represented the relationship between a drug and an ADR using a set of automatically extracted lexical patterns. We proposed a novel signal detection problem where given a social media post $T$ that contains a drug $D$ and an ADR $R$, we would like to determine whether $R$ is related to $D$, or otherwise. We then learn real-valued weights for the extracted lexical patterns that indicate their reliability for expressing an ADR of a given drug.

Results: We obtained an ADR detection accuracy of $74 \%$ on a large-scale manually annotated dataset of tweets, covering a standard set of drugs and ADRs. Moreover, intuitive lexical patterns that describe possibilities of the occurrence of an ADR were captured by the proposed method.

Conclusions: To the best of our knowledge, ours is the first causalitysensitive approach for detecting ADRs from social media. Our results show that the proposed method significantly outperforms several competitive baselines and a bag-of-words classifier. Moreover, both midfix as well as prefix information extracted from social media posts provide useful information for the classifier.

\section{Comparative Safety of Targeted Therapies for mCRC Treatment Between Young and Elderly Patients: a Study Using VigiBase}

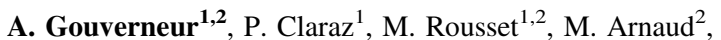
A. Fourrier-Réglat $^{1,2}$, A. Pariente ${ }^{1,2}$, T. Aparicio ${ }^{3}$, G. Miremont-Salamé ${ }^{1,2}$, P. Noize ${ }^{1,2}$

${ }^{1}$ CHU de Bordeaux, Pharmacologie Médicale, Centre de Pharmacovigilance, Bordeaux Cedex, France, ${ }^{2}$ Univ. Bordeaux, Inserm Bordeaux Population Health Research Center, team Pharmacoepidemiology, UMR 1219, Bordeaux Cedex, France, 
${ }^{3}$ Gastroenterology and Digestive Oncology department, Saint Louis Hospital, Paris, France

Background: Metastatic colorectal cancer (mCRC) is frequent among elderly, and often treated with targeted therapies. Yet, safety of those therapies is understudied in real-life, especially in the elderly.

Objective: To describe and compare according to age, the adverse events (AEs) reported for targeted therapies used for the treatment of mCRC in real-life setting.

Methods: An extraction of VigiBase, which contains WHO global individual case safety reports (ICSRs), was performed on the 1st December 2016. All reports of AEs with the following suspected targeted therapies (anti-VEGF: aflibercept, bevacizumab, regorafenib; anti-EGFR: cetuximab, panitumumab) and with mCRC as indication were considered. A descriptive analysis of AEs (according to MedDRA System Organ Class (SOC) and High Level Term (HLT) levels) was performed for all drugs and by therapeutic class. Chi-square tests were used to compare frequencies of $\mathrm{AE}$ between elderly ( $\geq 75$ years) and non-elderly adults ( $<75$ years).

Results: A total of 21,565 ICSRs were retrieved corresponding to 57,427 AEs. Among them, $74 \%$ were serious, and $11 \%$ fatal. Patient's median age was 64 years, $15 \%$ were aged $\geq 75$ and over, and $57 \%$ were male. Bevacizumab was suspected in $44 \%$ of ICSRs and cetuximab in $28 \%$. Cetuximab was significantly more reported in non-elderly adults whereas panitumumab was in elderly. Three SOCs were significantly more reported for both classes in elderly patients: "Cardiac disorders", "Metabolism and nutrition disorders", and "Respiratory, thoracic and mediastinal disorders"; one was in non-elderly adults: "Skin and subcutaneous tissue disorders". Two SOCs were significantly more reported for anti-VEGF in elderly patients: "Nervous system disorders" and "Infections and infestations" and two others in non-elderly adults: "Hepatobiliary disorders" and "Musculoskeletal and connective tissue disorders". For HLT, "Heart failures Not Elsewhere Classified (NEC)" was significantly most frequent for both classes in elderly while "Supraventricular arrhythmias" and "Ischaemic coronary artery disorders" were significantly most frequent for anti-VEGF in elderly and "Peripheral embolism and thrombosis" significantly more reported for anti-EGFR in non-elderly adults. "Non-site specific gastrointestinal haemorrhages" was significantly more reported for anti-VEGF in elderly; yet frequencies of "Haemorrhages NEC", and "Intestinal ulcers and perforation NEC" were not different for both classes among age groups. Thrombocytopenias" were significantly more reported for anti-VEGF in non-elderly adults.

Discussion: AEs related to targeted therapies used for the treatment of mCRC were different across groups. These differences should be taken into account for the prevention and surveillance of AEs according to age.

\section{Serious Drug Adverse Reactions: A Retrospective and Descriptive Study from the French regions of Bordeaux, Limoges, and Poitiers}

\author{
J. Perino ${ }^{1}$, A. Gouverneur ${ }^{1,2}$, M.L. Laroche ${ }^{3}$, \\ M.C. Perault-Pochat ${ }^{4,5}$, M. Allouchery ${ }^{4,5}$, H. Géniaux ${ }^{3}$, \\ G. Miremont-Salamé ${ }^{1,2}$, A. Pariente ${ }^{1,2}$
}

${ }^{1}$ CHU de Bordeaux, Pharmacologie Médicale, Centre de Pharmacovigilance, Bordeaux Cedex, France, ${ }^{2}$ Univ. Bordeaux, Inserm Bordeaux Population Health Research Center, team Pharmacoepidemiology, UMR 1219,Bordeaux Cedex, France, ${ }^{3} \mathrm{CHU}$ de Limoges, Service de Pharmacologie, Toxicologie et Pharmacovigilance, Centre de Pharmacovigilance,Limoges Cedex, France, ${ }^{4} \mathrm{CHU}$ de Poitiers, Pharmacologie clinique, Centre de
Pharmacovigilance,Poitiers Cedex, France, ${ }^{5}$ LNEC, INSERM U1084,Poitiers Cedex, France

Background: Serious adverse drug reactions (ADRs) represent an important public health problem because of individual health consequences and of economic impact. To allow better preventing these events, the description of the involved drugs, that change with market and uses, and that of concerned patients, need to be regularly updated.

Objective: To describe the characteristics of serious ADRs reported in Nouvelle Aquitaine (that covers the administrative areas of Bordeaux, Limoges, and Poitiers) over a nine-year period.

Methods: Information relative to the serious ADRs reported to the Pharmacovigilance Centers of Bordeaux, Limoges and Poitiers between 01/01/2007 and 01/01/2016 were extracted and analyzed. Serious ADRs were described in terms of event type (according to MedDRA SOC levels and related HLTs most frequently represented), suspected drugs, and patients' characteristics (age and sex). Descriptive analyses were performed for all serious ADRs with a focus on drug misuse; all results were stratified on age and sex.

Results: Overall, 16,243 reports of serious ADRs were identified. Patients' median age was 59.4 years; $53.1 \%$ were female (missing data: $0.4 \%$ of reports). Antineoplastic agents constituted the drugs most frequently incriminated (12.3\%), followed by antithrombotic agents (11.3\%) and systemic antibiotics (11.3\%). Results varied according to age. Analgesics were most represented in children (5.4\% in $0-15$ years); cardiac drug therapies were almost only found in the elders ( $3.2 \%$ in $\geq 75$ years). If importance of antithrombotics increased with age, antipsychotics agents were represented similarly across all age categories (around 5.6\%). Serious ADRs associated to misuse mostly concerned antithrombotic agents (16.7\%), analgesics (13.6\%) and psycholeptics (12.8\%). Reported serious ADRs concerned mainly nervous system disorders (11.9\%), skin and subcutaneous tissue disorders (11.6\%), and blood and lymphatic system disorders $(9.8 \%)$. Altogether, ADRs affecting these systems represented $33.3 \%$ of serious ADR reports. No significant difference was found according to sex for the type of serious ADRs. By contrast, age stratification showed that nervous system disorders (17.6\%) and skin and subcutaneous tissue disorders $(13.1 \%)$ were most represented in [0-15 years] whereas gastrointestinal disorders were most represented in elderly ( $11.7 \%$ in $\geq 75$ years). ADRs concerning blood and lymphatic system disorders increased with age.

Discussion: During the period considered, drugs most involved in serious ADRs were antineoplastic agents even though differences were found according to age. Specificities were found when focusing on serious ADR associated with misuse, highlighting the importance of antithrombotics. Nature of ADR differed according to age, confirming that extreme ages have peculiar sensibility to ADRs.

\section{Angiotensin II Receptor Blockers and Psoriasis: an Analysis of the French Pharmacovigilance Database}

\author{
B. Azzouz ${ }^{1}$, M. Abou Taam ${ }^{1}$, S. Calvo ${ }^{1}$, A. Morel $^{1}, \underline{\text { T. Trenque }}{ }^{1}$ \\ ${ }^{I}$ Regional Center of Pharmacovigilance and Pharmacoepidemiology, \\ Reims, France
}

Introduction: Psoriasis is a multifactorial chronic inflammatory skin disease that affects $2 \%$ of the global population. Angiotensin II receptor blockers (ARBs) widely used for the treatment of essential hypertension and heart failure, are occasionally reported to induce or aggravate 
psoriasis in the literature (1-4). Psoriasis is not mentioned in the Summary of Product Characteristics of ARBs.

Aim: To characterize this unexpected ADR.

Methods: We performed a retrospective study using the French Pharmacovigilance Database (FPVD). All notifications until February 2016 with the high level terms "psoriatic conditions and "psoriatic arthropathies" with ARBs suspected drugs were included. Demographic data, suspected drug, action taken with the suspected drug and ADR profile were analyzed for each notification.

Results: 89 cases of psoriasis in association of ARBs are collected in the FVPD. The average age is $66.03 \pm 12.64$ years (29-90 years), the sex ratio $\mathrm{M} / \mathrm{F}$ is $1.29 .42 .7 \%$ of cases are considered "serious" (38/89). There is no case of death. A medical history of "psoriasis" is mentioned in $39.3 \%$ of cases (35/89), these cases are those corresponding to the "aggravation of psoriasis". Time to onset is mentioned in $57.30 \%$ of cases (51/89), is in $78.43 \%$ of cases less than 1 year. ARBs are stopped in $52 \%$ of cases (46/89). After drug cessation, the outcome is favorable in $67 \%$ of cases (31/46), with no case of positive rechallenge. The ARBs are distributed as follows: valsartan $33.71 \%(\mathrm{n}=30)$, irbesartan $25.84 \%$ $(\mathrm{n}=23)$, losartan 16.85\% $(\mathrm{n}=15)$, candesartan $12.36 \% \quad(\mathrm{n}=11)$, telmisartan $5.62 \%(n=5)$, olmesartan $5.62 \%(n=5)$. In $36 \%$ of cases (32/89), ARBs are the only drugs suspected. In the cases with other suspected drugs (57/89), the most frequent therapeutic classes are diuretics $(63.1 \%, 36 / 57)$, beta-blockers $(26.3 \%, 15 / 57)$ and calcium channel blockers $(22.8 \%, 12 / 57)$.

Conclusions: To our knowledge this is the biggest case series described with ARBs and psoriasis. The fact that psoriasis is reported for all ARBs suggests a class effect. Increased keratinocyte proliferation as a result of elevated angiotensin II serum levels is the wide-spread mechanistic hypothesis (2). ADR characteristics (preponderance of induction cases, time to onset less than 1 year, positive dechallenge) are in line with the literature data (1-4). Considering this chronic and multifactorial disease, this unexpected ADR is probably underdiagnosed and underreported. Physicians must be aware of this side effect with this therapeutic class.

Further sources of information/References:

1. Lamba G, Palaniswamy C, Singh T, Shah D, Lal S, Vinnakota R, et al. Psoriasis induced by losartan therapy: a case report and review of the literature. Am J Ther 2011;18:e78-80

2. Marquart-Elbaz C, Grosshans E, Lipsker D, Lipsker D. Sartans, angiotensin II receptor antagonists, can induce psoriasis. $\mathrm{Br} \mathrm{J}$ Dermatol 2002;147:617-8

3. Kawamura A, Ochiai T. Candesartan cilexetil induced pustular psoriasis. Eur J Dermatol 2003;13:406-7

4. Keerthi S, Rangaraj M, Karthikeyan K. Telmisartan aggravates pustular psoriasis. J Pharmacol Pharmacother 2015;6:107-9

\section{Impact of Weekly Visits by Pharmacovigilance Center Pharmacist on Questions About Adverse Drug Reactions Asked by Intensive Care Unit practitioners}

\author{
C. Simon ${ }^{1}$, P. Asfar ${ }^{2}$, G. Drablier ${ }^{1}$, D. Bourneau Martin ${ }^{1}$, \\ M. Briet ${ }^{1}$, F. Beloncle ${ }^{2}$, L. Lagarce ${ }^{1}$
}

${ }^{1}$ Service de Pharmacologie Toxicologie et Pharmacovigilance CHU Angers, Angers, France, ${ }^{2}$ Département de Réanimation médicale et de Médecine Hyperbare CHU Angers, Angers, FRANCE

Introduction: One of the missions of the French Regional Pharmacovigilance Centre is to answer questions relating to Adverse Drug
Reactions (ADRs) asked by healthcare professionals. To facilitate notifications of adverse drug reactions, since June 2012 a pharmacist of the Regional Centre of Pharmacovigilance of Angers has been visiting the hospital medical Intensive Care Unit (ICU) once a week. This has helped strengthen the relationship between the ICU and the Pharmacovigilance Centre as well as promote discussions and questions.

Aim: To evaluate the impact of the pharmacist's visit on questions asked by ICU staff and to analyse these questions.

Methods: Questions asked by ICU staff to the CRPV between January 2012 and December 2016 were retrospectively collected and analysed using an Excel spreadsheet. Recorded data were: the number and subject of the questions, the type of ADRs, medicines, patient age and sex, and the request methods. The drugs were categorised using the ATC (Anatomical Therapeutic Clinical) classification and the ADRs using MedDRA (Medical Dictionary for Regulatory Activities).

Results: 109 questions on 334 drugs (1 to 12 per question) were asked during this period, with a significant increase in the number of questions from June 2012 (X9/year). Most questions were asked the pharmacist during the visit $(64 \%)$ or by phone $(32 \%)$, and often related to ADRs (89\%). The main System Organ Classes (SOCs) involved were the nervous system $(14.7 \%)$, the respiratory system $(14.7 \%)$, the vascular system (10\%), and the hematologic system (8.2\%). The most commonly discussed drugs were psychotropic drugs (23.7\%), anti-infective drugs (15.6\%), medication for the alimentary tract and metabolism (14.4\%), and cardiovascular drugs (12\%).

Discussion/conclusion: Drugs and ADRs were varied, which reflected the range of diseases treated in the ICU. Some ADRs occurred in the ICU, but questions also related to patients who were admitted to the ICU due to an ADR. In 2012, visit arrangements were discussed between intensive care service and pharmacovigilance practitioners to implement the best conditions that would allow to promote ADR declarations without disrupting service organization and patient care. This collaborative approach has led not only to a significant increase of declarations, but also to a rise in questions about ADRs as well as dialogue improvement between the ICU and the Pharmacovigilance Centre, thus enhancing ADRs diagnosis and care.

\section{Self-Medication During the Third Trimester of Pregnancy: A Retrospective Study}

\author{
A. Monnier ${ }^{2}$, C. Gaudin ${ }^{2}$, C. Rouillard ${ }^{2}$, G. Drablier ${ }^{1}$,
} D. Bourneau Martin ${ }^{1}$, M. Briet ${ }^{1}$, L. Lagarce ${ }^{1}$

${ }^{1}$ Service de Pharmacologie Toxicologie et Pharmacovigilance CHU Angers, Angers, France, ${ }^{2}$ Ecole de sage-femme du CHU d'Angers, département de maïeutique de l'UFR santé de l'Université d'Angers, Angers, France

Background: Self-medication is a common practice in our society, even during pregnancy. However, during this period, some products are not recommended, or even not allowed, due to the embryo-foetal risks. Previous French studies have shown a high prevalence of self-medication during pregnancy [1-2] as well as a lack of awareness of drug side effects by pregnant women, especially regarding the risks of taking non-steroidal anti-inflammatory medications during the third trimester of pregnancy [3]. Objective: The main objective was to estimate the prevalence of selfmedication during the third trimester of pregnancy. The secondary aims were to describe patient characteristics, the drugs used, the perception of drug risk and the contributing factors of this practice in order to offer appropriate safety precautions. 
Method: An observational, descriptive, retrospective, quantitative, and monocentric study carried out at Angers University Hospital between 3 December 2016 and 7 January 2017. Data was collected using a questionnaire completed by adult women who gave birth at term during this period. Data was analysed using Sphinx Plus software.

Results: 293 questionnaires were analysed. The prevalence of self-medication during the third trimester of pregnancy was $22.9 \%$. Factors associated with more frequent use of self-medication were: employment in healthcare $(\mathrm{p}=0.004)$; alcohol consumption during pregnancy $(\mathrm{p}=0.04)$; and the use of self-medication before pregnancy $(\mathrm{p}<0.001)$. The main conditions treated were headaches (38.5\%); coughs, sore throats and influenza-like illnesses (29.8\%); gastroesophageal reflux (19.4\%); and abdominal pain (16.4\%). The most often used drugs were acetaminophen (44.8\%), homeopathy (23.9\%), phloroglucinol (14.9\%), and anti-acid drugs (14.9\%), none of which were known to be toxic for the foetus. Although most of the women questioned $(68.5 \%)$ received information about the drugs during pregnancy, $25.6 \%$ were unaware of the potential risks of certain over-the-counter products.

Discussion/Conclusion: In this study, the prevalence of self-medication was lower than in previous studies, however the studied period was different, given that it related solely to the third trimester. The results of the study are reassuring, although a lack of awareness shown by $25 \%$ of the patients is worrying. As such, preventive measures should be implemented to inform women about the risks of self-medication during pregnancy.

Further sources of information/References:

1. Courrier D, Villier C, Jourdan S, Hoffmann P. Self-medication and pregnancy: Survey of 740 pregnant women enrolled in the perinatal network of the Alpes-Isere region in France. Rev Sage-femme 2015; 14:131-41

2. Schmitt B. L'automédication chez la femme enceinte.J Gynecol Obstet Biol Reprod 2002;31:211

3. Hassoun-Barhamji R, Raia Barjat $T$, Chauleur C. A l'ère de l'automédication, que savent les femmes enceintes des anti-inflammatoires? Thérapie 2015;70:369-76

\section{The Occurrence of ADRs of ADHD Medication in the Adult Population Collaboration with Patient Organisation Contributing to Medicine Safety}

\author{
$\underline{\text { G. Weits }^{1}}$, L. Härmark ${ }^{1}$, J. Hartman ${ }^{1}$, A.C. Kant ${ }^{1}$ \\ ${ }^{I}$ Netherlands Pharmacovigilance Centre Lareb, 's-Hertogenbosch, \\ the Netherlands
}

Background: Methylphenidate, dexamphetamine and atomoxetine are used for treatment of $\mathrm{AD}(\mathrm{H}) \mathrm{D}$ in the Netherlands [1]. Of these three, only atomoxetine is registered for starting treatment of $\mathrm{AD}(\mathrm{H}) \mathrm{D}$ in adults. Therefore prescribing medication for $\mathrm{AD}(\mathrm{H}) \mathrm{D}$ to adults is mostly offlabel. Also, little is known about drug use and adverse drug reactions (ADRs) in adults with $\mathrm{AD}(\mathrm{H}) \mathrm{D}$. Both issues where highlighted by the Dutch patient organisation for adults with $\mathrm{AD}(\mathrm{H}) \mathrm{D}$, which resulted in an active collaboration between the Netherlands Pharmacovigilance Centre Lareb the patient organisation.

Aim: To investigate the use and experiences with ADHD medication, including ADRs, in the elderly.

Methods: A web-based questionnaire was developed, containing various domains: patient characteristics, diagnosis and medication use, the satisfaction around provided drug information, efficacy of the drug, reasons for drug discontinuation, ADRs and experiences with drug substitution. The questionnaire was distributed to members of the patient organisation, members of the internet forum ADHD-Xtra and via healthcare professionals that are members of the ADHD network. The mentioned ADRs were coded using the MedDRA terminology. All patients who mentioned the occurrence of a possible ADR were asked to report this ADR to Lareb separately.

Results: 848 valid questionnaires were received. $76.2 \%(n=646)$ of the respondents indicated that they used one or more drugs for the treatment of their $\mathrm{AD}(\mathrm{H}) \mathrm{D}$. The most mentioned drugs were long- and short-acting methylphenidate respectively. Of the respondents, $74.1 \%(\mathrm{n}=479)$ said that they experienced a positive effect from the drug on their $\mathrm{AD}(\mathrm{H}) \mathrm{D}$ symptoms and $75.2 \%(n=620)$ experienced ADRs. Also, $47.6 \%(n=404)$ of the respondents reported that they had stopped using their medication at one point; in $59.7 \%(n=241)$ this was due to the occurrence of ADRs. In total, 1857 ADRs were mentioned, see table 1. Mentioned ADRs of interest were a 'rebound effect' with methylphenidate, an increased susceptibility to the effects of alcohol and an increased craving for nicotine. 44 respondents reported their ADRs via the official reporting form to Lareb.

Conclusion: Most respondents experience a positive effect of their drugs, but about the same number also experiences ADRs. The occurrence of ADRs was the main reason for respondents to stop their treatment. Our study shows that active collaboration with a patient organisation increases the knowledge about drug use and ADRs in a selected population. The collaboration also increases the number of reports submitted to the spontaneous reporting system and raises awareness about pharmacovigilance among the general public.

Further sources of information/References:

1. Farmacotherapeutisch Kompas. ADHD bij kinderen. (version date: 26-4-2017, access date: 4-5-2017). Available from: https://www. farmacotherapeutischkompas.nl/bladeren/indicatieteksten/adhd_bij_ kinderen

Table 1 Top 10 reported ADRs for methylphenidate and dexamphetamine

\begin{tabular}{llll}
\hline ADRs methylphenidate & Number & ADRs dexamphetamine & Number \\
\hline Palpitations & 117 & Dry mouth & 24 \\
Decreased appetite $^{3}$ & 117 & Palpitations & 22 \\
Rebound effect $^{3}$ & 103 & Feeling jittery & 21 \\
Headache & 101 & Sleep disorder & 18 \\
Feeling jittery & 80 & Decreased appetite & 14 \\
Apathy & 70 & Headache & 12 \\
Dry mouth & 70 & Depressed mood & 9 \\
Depressed mood & 54 & Apathy & 8 \\
Sleep disorder & 48 & Insomnia & 8 \\
Weight decreased & 43 & Tic & 7
\end{tabular}

${ }^{3}$ Rebound effect: symptoms are (stronger) back when the drugs are developed. This is mainly seen in short-acting medication. When long-acting medications this effect is less and need medication usually only be taken once a day.

\section{Number Needed to Treat to Harm (nnth) to Assess the Risk of Cataracts Associated with Statins in Observational Studies}

\author{
D. Mendes ${ }^{1,2}$, C. Alves ${ }^{1,2}$, F. Batel Marques ${ }^{1,2}$
}

${ }^{1}$ Coimbra Regional Pharmacovigilance Unit, Centre for Health Technology Assessment and Drug Research, Association for Innovation and Biomedical Research on Light and Image, Coimbra, Portugal, ${ }^{2}$ University of Coimbra, School of Pharmacy, Coimbra, Portugal

Background: Observational studies have pointed out statistically significant increased risk of cataracts in patients receiving statin therapy [1-3]. The clinical relevance of this finding deserves investigation. The number 
needed to treat to harm (NNTH) provides quantification of risks in an absolute scale. This metric may add value to treatment decisions, by telling clinicians how many patients they need to treat so that one will have the event of interest over a period of time.

Aim: This study aimed to evaluate the risk of cataracts in patients receiving statins, using the NNTH.

Methods: A literature search was conducted in Pubmed and EMBASE until December 2016. Observational comparative studies evaluating the risk of cataracts in patients receiving statins were included. Odds ratios (ORs) were calculated using a random-effects model meta-analysis. $\mathrm{I}^{2}$ test assessed heterogeneity. Sensitivity analyses were conducted to test the influence of the following variables in the risk estimate: (1) each individual study; (2) risk factors for cataracts (diabetes, age, gender, smoking, cardiovascular disease, steroids); (3) studies' design. NNTHs were calculated applying statistically significant ORs to the five-year incidence rate of cataract surgeries (10.2\%) in AREDS Study [4].

Results: Twenty observational studies (10 cohorts, 7 case-controls and 3 cross-sectionals) were retrieved from 19 publications. Overall, treatment with statins was associated with an increased risk of cataracts [OR $1.11(95 \% \mathrm{CI}$ $\left.1.02-1.21) ; \mathrm{I}^{2}=97.4 \%\right]$. The NNTH was estimated at 101 (95\% CI 54-547) over five years of treatment. Sensitivity analyses demonstrated the following: (1) when individually removed, only one study leads to the loss of statistical significance of the result; (2) overall risk estimate was not influenced by risk factors for cataract; (3) cohorts: OR 1.09 (95\% CI 0.94-1.26); $\mathrm{I}^{2}=98.2 \%$; case-controls: OR 1.14 (95\% CI 1.04-1.24); $\mathrm{I}^{2}=94.1 \%$; NNTH 80 (95\% CI 47-275); cross-sectionals: OR 1.09 (95\% CI 0.72-1.66); $\mathrm{I}^{2}=78.6 \%$ ).

Conclusions: An increased risk of cataracts was found to be associated to the use of statins. However, these results should be interpreted carefully since the heterogeneity was high, and there was loss of statistical significance in few analyses. Moreover, the risk should be weighed against the known and well-documented benefits of statins, in the light that the NNT to prevent death and non-fatal heart attack was calculated as 83 and 39, respectively, within 5 years, in patients with heart disease [5]. These findings deserve further attention and research.

Further sources of information/References

1. Erie JC, et al. Statin Use and Incident Cataract Surgery: A CaseControl Study. Ophthalmic Epidemiol 2016;23:40-5

2. Casula M, et al. Statin use and risk of cataract: A nested case-control study within a healthcare database. Atherosclerosis 2016;251:153-8

3. Wise SJ, et al. Statin use and risk for cataract: a nested case-control study of 2 populations in Canada and the United States. Can J Cardiol 2014;30):1613-9

4. Koo E, et al. Ten-year incidence rates of age-related cataract in the Age-Related Eye Disease Study (AREDS): AREDS report no. 33. Ophthalmic Epidemiol 2013;20:71-81

5. Newman, D. Statins Given for 5 Years for Heart Disease Prevention (With Known Heart Disease). Published/Updated: November 2, 2013. Available from: http://www.thennt.com/nnt/statins-for-heart-diseaseprevention-with-known-heart-disease/. Accessed April 26, 2017

Abbreviations

AREDS, Age-Related Eye Disease Study.

\section{Bogota Declaration: The Intention to Promote Both Cooperation Concerning Safe Use of Medicines and Pharmacovigilance Development in Latin America}

\author{
R.A. Caro-Rojas ${ }^{1}$, C. Maldonado Muete ${ }^{1}$ \\ ${ }^{1}$ Colombian Pharmacovigilance Association, Bogotá, Colombia
}

Background: The Safe use of Medicines has recently been reemphasized by the World Health Organization through a global initiative to reduce medication errors by $50 \%$ in 5 years. In Latin America, different institutions and networks have implemented isolated strategies, plans and tools that greater cooperation must be promoted based on common aims.

Objective: To describe how the "Bogotá Declaration for Latin America" may act as a regional agreement as a basis for achieving safer use of medicines.

Methods: Colombian Pharmacovigilance Association (ACFV) has promoted an agreement calling for greater cooperation between Latin American countries to drive safer use of medicines and pharmacovigilance development. This agreement was signed by participants in the 3rd Annual Meeting of the Latin American Chapter of ISOP (Bogota, Colombia, August 2016) who came from Brazil, Chile, Mexico, Colombia and Central America as well as representatives of ISOP and the Uppsala Monitoring Center.

Results: The final document included the following Objectives:

- To generate proposals for the harmonization of Pharmacovigilance in Latin America.

- To study the impact of pharmacovigilance on public health and the management and sustainability of health systems.

- To make all different parties more aware about the impact of Pharmacovigilance.

- Promote active participation in national and international arenas where this subject is raised.

- To promote the culture of safe use of medications, risk communication and pharmacovigilance training

- To facilitate the establishment of the guiding principles regarding Pharmacovigilance for the region.

- To promote spaces for patient and community participation, in programs of Pharmacovigilance and medication safe use.

The Declaration has been widely disseminated targeting key PV players such as: Regulatory authorities, health organizations and providers, academia, industry and patients.

The document has been disseminated through social networks and face-toface meetings including Colombia, Peru, Uruguay and Ecuador. South American Pharmaceutical Care Network, Pharmacovigilance Latin American Network, Pharmaceutical Care Network of Ecuador, Medication Safe Use Latin America Network and Patient Safety groups. The Declaration was announced in the Uppsala Reports Bulletin and ISOP website. We estimate the Declaration has directly reached 10,000 people in the PV community although it is not possible to be precise about actual numbers. Discussion: By providing an agreed framework to form the basis of further action, the Bogota Declaration has been an effective tool for engaging PV practitioners to promote cooperation.

Conclusion: Dissemination of the Declaration should continue. Further studies are required to measure the impact of cooperation.

\section{Safety of BRAF and MEK Inhibitors for Treatment of Metastatic melanoma: Analysis of the WHO Database of Adverse Events}

M. Rousset ${ }^{1,2}$, A. Gouverneur ${ }^{1,2}$, M. Arnaud ${ }^{2}$, P. Claraz ${ }^{1}$, A. Fourrier-Réglat ${ }^{1,2}$, A. Pariente ${ }^{1,2}$, G. Miremont-Salamé ${ }^{1}$, M. Molimard ${ }^{1,2}$

${ }^{1}$ Service de Pharmacologie Médicale, Centre Hospitalier Universitaire de Bordeaux, Bordeaux, France, ${ }^{2}$ Univ. Bordeaux, Inserm, Bordeaux Population Health Research Center, Team Pharmacoepidemiology, UMR 1219, Bordeaux, France 
Background: Bitherapy by BRAF- and MEK-inhibitors provides significant benefits in BRAFV600 ${ }^{\text {mut }}$ metastatic melanoma patients; however adverse events (AEs) occur in $90 \%$ of treated patients in clinical trials. What about AE patterns in real life?

Objective: We aimed to describe AEs profiles of BRAF-inhibitors (vemurafenib, dabrafenib) and MEK-inhibitors (cobimetinib, trametinib) in metastatic melanoma treatment in real life settings.

Methods: Global individual case safety reports (ICSRs) were extracted from VigiBase ${ }^{\circledR}$ on December 2016 the 1st. Inclusion criteria were: (1) at least one adverse event with the following suspected targeted therapies (vemurafenib, dabrafenib, cobimetinib, trametinib), (2) indication of metastatic melanoma. A descriptive analysis of AEs according to MedDRA High Level Terms was performed.

Results: We retrieved 8041 ICSRs corresponding to 22,270 AEs. $75 \%$ of ICSRs were serious and $12 \%$ led to death. We identified 301 ICSRs for vemurafenib + cobimetinib in bitherapy; 2287 for dabrafenib + trametinib in bitherapy; 4060 for vemurafenib; 66 for cobimetinib; 819 for dabrafenib, and 508 for trametinib. Patients' median age was 59 years; $56 \%$ were male.

AEs common to the 4 drugs were: general signs and symptoms Not Elsewhere Classified (NEC); rashes, eruptions and exanthems NEC; asthenic conditions; nausea and vomiting symptoms; diarrhoea; febrile disorders; arthralgia; skin neoplasms malignant and unspecified; neoplasms malignant site unspecified NEC; metastases to specified sites; death and sudden death. Focusing on reported AEs with a frequency greater than $1 \%$, photosensitivity and photodermatosis conditions $(\mathrm{n}=29)$; renal failure and impairment $(\mathrm{n}=24)$ and alopecias $(\mathrm{n}=11)$ were reported only with the bitherapy vemurafenib + cobimetinib whereas feelings and sensations NEC $(\mathrm{n}=227)$, visual disorders NEC $(\mathrm{n}=83)$ and headaches NEC $(n=82)$ were reported only with the bitherapy dabrafenib + trametinib. Appetite disorders $(\mathrm{n}=202)$, erythemas $(\mathrm{n}=210)$, pruritus NEC $(\mathrm{n}=158)$ and bullous conditions $(\mathrm{n}=133)$ were reported more frequently with vemurafenib. Musculoskeletal and connective tissue pain and discomfort $(n=191)$; pain and discomfort NEC $(n=81)$ were more often reported with dabrafenib. Skeletal and cardiac muscle analyses $(n=25)$; acnes $(n=16)$; hepatocellular damage and hepatitis NEC $(n=13)$ and oedema NEC $(n=12)$ were reported more often with cobimetinib.

Conclusion: Beyond the common base of AEs to these targeted therapies, AEs profiles differed between bitherapies and between molecules in reallife settings. These differences should be taken into account for the prevention and surveillance of AEs.

\section{Ladies' territory? Patient Sex Distribution in a Global Database of Suspected Adverse Drug Reactions}

\author{
S. Watson ${ }^{1}$, K. Star ${ }^{1,2}$, Y. Aoki ${ }^{1}$, P. Caduff-Janosa ${ }^{1}$ \\ ${ }^{1}$ WHO Collaborating Centre for International Drug Monitoring, \\ Uppsala Monitoring Centre, Uppsala, Sweden, ${ }^{2}$ Department of Public \\ Health and Caring Sciences, Uppsala University, Uppsala, Sweden
}

Background: There are differences between the type of drugs used by males and females and the susceptibility and nature of suspected adverse drug reactions (ADRs) [1,2]. It has also previously been shown that there is a difference in reporting patterns between males and females in spontaneous ADR reports collected internationally [3]. However, there is limited information about the present situation.
Objective/Aim: To investigate the current reporting patterns for females in a global database of spontaneous ADR reports.

Methods: Features of ADR reports for females and males in VigiBase were compared and categorised over time, within age groups, type of reporters and reporting countries. The dataset from April 2017 consisted of thirteen million reports originating from 125 countries participating in the WHO Programme for International Drug Monitoring.

Results: The proportion of female reports in VigiBase overall has been constantly at around $60 \%$ since the programme started in 1968. Up until the age of 11 years, female reports are underrepresented with $46 \%$ for each of the respective age groups; 0-27 days, 28 days -23 months, $2-11$ years, whilst reports for females dominate in all age groups above; $12-17(58 \%)$, 18-44 (66\%), 45-64 (60\%), 65-74 (56\%), 75 and above (59\%). All types of reporters submit slightly more reports for females but the largest difference is seen when patients themselves report (64\%). Among countries with at least 500 reports submitted to VigiBase, only India (49\% female reports), Andorra (45\%) and Macedonia (33\%) have submitted more male reports.

Conclusion: Although there are more males than females in the world, we observe more ADR reports from females in VigiBase from the age of 12 years. This is consistent with previous findings in both VigiBase and elsewhere [3,4]. So, what is happening with females in puberty? Do women experience more ADRs or do the just report more diligently? There are studies pointing in both directions [4,5] and it has also been shown that puberty and the consequent influence of hormones play a key role in the development of certain ADRs [6]. The higher proportion of female reports in international ADR surveillance is not limited to a specific subset of the database or point in time. The general trend with more female reports highlights the need for further research within this field.

\section{Further sources of information/References:}

1. Kando JC, Yonkers KA, Cole JO. Gender as a risk factor for adverse events to medications. Drugs 1995;50:1-6

2. Franconi F, et al. Gender differences in drug responses. Pharmacol Res 2007; 55: 8195

3. Fridén S. Star K, Norén N. Gender differences in international adverse drug reaction surveillance [ISOP Abstract 2009], Drug Saf. 2009;32:929.

4. Martin RM, et al. Age and sex distribution of suspected adverse drug reactions to newly marketed drugs in general practice in England: analysis of 48 cohort studies. Br J Clin Pharmacol 1998; 46:505-11

5. Almeida L, et al. Personality characteristics of volunteers in Phase 1 studies and likelihood of reporting adverse events. Int J Clin Pharmacol Ther 2008; 46:340-8

6. Drici M-D,Clément N. Is Gender a Risk Factor for Adverse Drug Reactions? The Example of Drug-Induced Long QT Syndrome. Drug Safety 2001;24:575-585

\section{Safety of the Hexavalent Vaccine as Used in General Practice: Interim Results from a Prospective Observational Study}

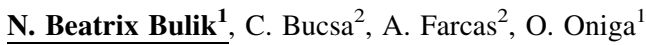 \\ ${ }^{1}$ Pharmaceutical Chemistry Department, Iuliu Hatieganu University \\ Of Medicine And Pharmacy, Cluj-Napoca, Romania, ${ }^{2}$ Drug \\ Information Research Center, Iuliu Hatieganu University Of \\ Medicine And Pharmacy, Cluj-Napoca, Romania
}


Background: The confidence in immunization via vaccination is low in many European countries due the distrust in vaccines' safety [1].

Aim: To present the interim results of an ongoing study that is assessing the safety of the hexavalent vaccine (diphtheria-tetanus-pertussis toxoid, inactivated poliomyelitis virus, antigens against hepatitis B and Haemophilus influenza type B polysaccharide) used in general practice in Romania.

Methods: We performed a prospective observational study to assess the safety of hexavalent vaccine and other pediatric vaccines administered to children up to 7 years according to the national immunization schedule in 2 general practitioners (GPs) office. Baseline data was collected on demographic characteristics, medical history, history of adverse events occurred after prior vaccination, prior use of medication. After vaccination, we collected data regarding reactogenicity (pain, redness and swelling at the injection site, fever, fussiness, drowsiness, loss of appetite, diarrhea, vomiting) within 4 days and any adverse event (AE) occurring within 6 months after vaccination that urged a visit to physician.

Results: The interim cohort consists in 147 children who received the hexavalent vaccine alone. The Hexacima ${ }^{\circledR}$ vaccine was administered in most of the children (94.5\% of 181 administered doses). The median age of vaccination was 6 months [2-17] and 54.4\% of the children were female. The most frequently reported solicited local reaction was pain (27.6\%) followed by redness $(21 \%)$. Of systemic adverse reactions, irritability was reported most frequently (48.6\%). Fever occurred in $15.5 \%$ of children while no subject reported Grade $3\left(>40{ }^{\circ} \mathrm{C}\right)$ fever. $27.9 \%$ of subjects reported at least 1 unsolicited AE (causality not implied). No death was reported.

Conclusions: The interim results showed that up to this point the hexavalent vaccine was well tolerated.

Further sources of information/References

1. Larson HJ, de Figueiredo A, Xiahong Z, Schulz WS, Verger P, Johnston IG, et al. The State of Vaccine Confidence 2016: Global Insights Through a 67-Country Survey. EBioMedicine 2016;12:295-301

\section{Natalizumab and rapidly evolving central nervous system lymphoma in VigiBase}

\author{
D. Sartori ${ }^{1}$, C. Westerberg ${ }^{1}$, B. Grundmark ${ }^{1,2}$
}

${ }^{1}$ WHO Collaborating Centre For International Drug Monitoring, Uppsala Monitoring Centre, Uppsala, Sweden, ${ }^{2}$ Department of Surgery, Uppsala University, Uppsala, Sweden

Background: Natalizumab, an $\alpha 4 b 1$-integrin inhibitor, limits lymphocyte passage through the blood-brain barrier. It is approved for the treatment of relapsing-remitting multiple sclerosis (RRMS) via monthly infusions. Central nervous system lymphoma (CNSL) is rare, with incidence increasing with age, particularly after age 55 [1]. Risk factors include immunosuppression and EBV infection. MS and CNSL may overlap and biopsy is preferred for diagnosis, imaging being less reliable [2]. A 2-year natalizumab study showed no impact on incidence of malignancies [3]. Aim: To assess the drug-adverse event combination (DEC) natalizumab with MedDRA PT "Central nervous system lymphoma" in VigiBase, alongside published literature cases.

Methods: Reports of the DEC until May 2015 were extracted from VigiBase. Literature cases were matched and duplicates removed. Clinical findings, co-reported terms and drugs, duration of treatment were appraised.
Results: There were 12 cases whereof 5 from literature with a n IC of 3.12 indicating the DEC was occurring more often than expected. There were eight females and four males; age range: 28-59 years (median 44). Natalizumab was the only suspect in 11, co-suspect in one with other immunosuppressants. Duration of treatment ranged from 1 to 21 cycles (median 3). Six cases were confirmed by biopsy, 3 patients were EBV/HIV negative, with the rest having unknown EBV/HIV status. Two were first misdiagnosed with MS but after 3 courses the diagnosis was changed to CNSL. In another case of a 41-year-old female, the perceived "MS" lesions grew rapidly after 2 courses raising suspicion of the later diagnosed CNSL.

Two literature cases of patients were outside the expected CNSL age range; a 40-year-old male diagnosed with RRMS via MRI, CSF analysis and biopsy. Azathioprine treatment failure was followed by natalizumab with a CNSL diagnosis after 21 courses. EBV and HIV tests were negative [4]. A second EBV negative 40-year-old male was diagnosed with CNSL after two courses of natalizumab; authors postulate that natalizumab may promote the progression of pre-existing lesions [5].

Conclusion: Several VigiBase case reports present similarities with the well described literature cases, being EBV/HIV negative and presenting with rapid lymphoma progression. Reduced lymphocyte CNS surveillance, mediated by natalizumab mechanism of action, may accelerate preexisting CNSL growth. Despite uncertainties regarding a causal role of natalizumab [6], we believe VigiBase data adds sufficient evidence to discuss an update to the natalizumab safety profile.

Foot note: as of 04-2017 an additional 13 cases, still under assessment by UMC, have been reported to VigiBase

Further sources of information/References:

1. Ostrom QT, Gittleman H, Liao P, Rouse C, Chen Y, Dowling J, et al. CBTRUS Statistical Report: Primary Brain and Central Nervous System Tumors Diagnosed in the United States in 2007-2011. NeuroOncology 2014; 16(Suppl 4):iv1-iv63

2. Hunt MA, Jahnke K, Murillo TP, Neuwelt EA. Distinguishing primary central nervous system lymphoma from other central nervous system diseases: a neurosurgical perspective on diagnostic dilemmas and approaches. Neurosurg Focus 2006; 21:E3

3. Polman CH, O'Connor PW, Havrdova E, Hutchinson M, Kappos L, Miller DH, et al. A Randomized, Placebo-Controlled Trial of Natalizumab for Relapsing Multiple Sclerosis. N Engl J Med 2006;354: 899-910

4. Schweikert A, Kremer M, Ringel F, Liebig T, Duyster J, Stüve O, et al. Primary central nervous system lymphoma in a patient treated with natalizumab. Ann Neurol 2009; 66:403-6

5. Phan-Ba R, Bisig B, Deprez M, De Prijck B, Delrue G, Herens C, et al. Primary central nervous system lymphoma in a patient treated with Natalizumab. Ann Neurol 2011; 69:1060-1

6. Bozic C, LaGuette J, Panzara MA, Sandrock AW. Natalizumab and central nervous system lymphoma: No clear association. Ann Neurol 2009; 66:261-2

\section{A Further Case Report of Hepatotoxicity Induced by Carbimazole with a Positive Rechallenge}

C. Maucorps ${ }^{1}$, V. Chawadronow ${ }^{1}$, X. Humbert ${ }^{1}$, B. Chrétien ${ }^{1}$, S. Fedrizzi

${ }^{I}$ Department of Pharmacology, CHU de la Côte de Nacre, Caen France 
Background: Beside withdrawal amiodarone treatment, hyperthyroidism induced by this drug may need treatment by antithyroid drug (AD) such as carbimazole known to rarely cause hepatotoxicity.

Objective: To present a further case of a patient treated by carbimazole which led to a symptomatic liver injury associated with a new case of positive rechallenge.

Methods: A detailed case report was done after a spontaneous notification to pharmacovigilance.

Results: The case is about a 76-year-old woman previously treated by amiodarone for 2 months and then switched by verapamil because of iatrogenic hyperthyroidism. As a treatment, carbimazole began at a dose of $20 \mathrm{mg}$ per day. After 7 months of treatment, her doctor sent her to emergency for icterus and a diffuse pruritus developing for few days. On physical examination, the patient had a high abdominal pain in the right upper quadrant and she presented a conjunctive and cutaneous jaundice. Laboratory testing showed elevations in conjugated bilirubine at $62 \mu \mathrm{mol} /$ $\mathrm{L}(\mathrm{N}<3 \mu \mathrm{mol} / \mathrm{L})$ and total bilirubin elevated to $100 \mu \mathrm{mol} / \mathrm{L}(\mathrm{N}=1-17$ $\mu \mathrm{mol} / \mathrm{L})$. Test revealed a cholestatic pattern with y-glutamyl-transferase at $1167 \mathrm{UI} / \mathrm{L}(\mathrm{N}=5-38 \mathrm{UI} / \mathrm{L})$, alkaline phosphatase at $837 \mathrm{UI} / \mathrm{L}$ ( $\mathrm{N}=35-90 \quad \mathrm{UI} / \mathrm{L})$ and a high elevation of transaminases with $\mathrm{TGO}=231 \mathrm{UI} / \mathrm{L}(\mathrm{N}=5-31 \mathrm{UI} / \mathrm{L})$ and TGP $=321 \mathrm{UI} / \mathrm{L}(\mathrm{N}=5-34 \mathrm{UI} /$ L). Anamnesis found that carbimazole dosage was increased to $40 \mathrm{mg}$ per day for a week. Carbimazole was withdrawn and a corticotherapy started. Other medications were continued. Six days later, laboratory parameters decreased clearly toward normal values and the patient was discharged. However, the patient takes inadvertently $40 \mathrm{mg}$ of carbimazole the morning after. $4 \mathrm{~h}$ later, she presented again to emergency with epigastric pain which ceased half-a-day later corresponding approximately to elimination of carbimazole. Laboratory test showed a greater increased of hepatic enzymes than previously described. The patient was allowed to go back home after two days of hospitalization.

Conclusion/Discussion: In this case, Naranjo probability scale indicated probable relationship between hepatotoxicity and the AD. This case summarizes liver toxicity of carbimazole which is dose dependent and associated to cholestasis [1]. Immediate discontinuation of antithyroid therapy is required. Laboratory tests could be proposed in case of necessity to increase dose in parallel to thyroid hormones analyses. After a first episode, medical advices should ensure to prevent the risk of rechallenge. For reasons of hepatotoxicity of other $\mathrm{AD}$, no other drug was prescribed. Nevertheless, pharmacovigilance indicated to the clinician there were potentially no cross reactivity between $\mathrm{AD}$ [2]; a propylthiouracile treatment was begun

Further sources of information/References:

1. Mikhail, NE. Methimazole-induced cholestatic jaundice. South Med J 2004; 97:178-183

2. Arab, DM, Malatjalian DA, Rittmaster RS. Severe cholestatic jaundice in uncomplicated hyperthyroidism treated with methimazole. J Clin Endocrinol Metab 1995; 80:1083-1085

\section{An Unexpected Digoxin Overdose Probably Caused by a Drug-Drug-Interaction after Amiodarone Withdrawal and Apixaban Initiation: a Case Report}

\author{
C. Maucorps ${ }^{1}$, V. Chawadronow ${ }^{1}$, S. Fedrizzi ${ }^{1}$, B. Chrétien ${ }^{1}$ \\ ${ }^{1}$ Department of Pharmacology, CHU de la Côte de Nacre, Caen, \\ France
}

Introduction: Digoxin is a substrate of a saturable efflux pump called P-Glycoprotein (P-gp) and which is considered as the main drug-metabolic pathway. P-gp substrates and P-gp inhibitors influence therefore the drug resorption [1]. Due to the narrow therapeutic window of digoxin, drugdrug-interactions (DDI) can potentially increase the risk of overdose.

Aim: To describe an unknown situation of DDI that could explain a digoxin overdose.

Methods: We present a pharmacovigilance case of digoxin overdose probably caused by a DDI with apixaban after a recent amiodarone withdrawal.

Results: A 89-years-old woman with a medical history included diffuse arthrosis, asthma and atrial fibrillation treated by amiodarone (100 mg-0$0)$ since 2000s. She presented in emergency for orthopnea and dyspnea. Further examination led to the diagnosis of tachyarrhythmia and cardiac insufficiency. Amiodarone was withdrawn and a treatment by intravenous (iv) digoxin and furosemide was initiated. After 3 days, iv digoxin was switched to oral route $(0.125 \mathrm{mg}$ per day). A regular heart rhythm was obtained $(\mathrm{N}=60-80 \mathrm{bpm})$. Digoxinemia (D) was done every 2 days until a steady-state concentration (SSC) in the therapeutic range (D $=0.7 \mathrm{ng}$ ) $\mathrm{ml}, \mathrm{N}=0.5-0.8 \mathrm{ng} / \mathrm{ml}$ ) was obtained on day 9 . In the mean time, apixaban was started at $2.5 \mathrm{mg}$ twice daily (on day 7). On day 12, digoxinemia increased to reach a maximum of $5.0 \mathrm{ng} / \mathrm{ml}$ which is strongly over the recommended therapeutic range for the elderly. There was no kidney injury $(\mathrm{GFR}=78 \mathrm{ml} / \mathrm{min}$ ), no hepatic injury and no new electrolytes variations that could explain this overdose [2]. No drug administration error nor drug initiation were detected by the medical staff. In front of this episode, no explanation was found but a pharmacovigilance survey considered as probable (based on Naranjo Scale) the onset of a drug-drug interaction.

Conclusion/Discussion: Amiodarone is well known as a powerful P-gp and CYP3A4 inhibitor and may induce interaction with other drug even many days after withdrawal because of its relatively long half-life. In this situation, P-gp activity was probably low because of amiodarone inhibition. Concentration of apixaban was increased by the same mechanisms. Theses elements may have led to a defective coverage of digoxin exposing the patient to face a digoxin overdose despite SSC obtained few days earlier. Although experiences didn't conclude with a significant modification of digoxin pharmacokinetic with the concomitant use of apixaban, a DDI may occur and lead to digoxin overdose when this drug is introduced after an amiodarone withdrawal.

Further sources of information/References:

1. Sharom, FJ. The P-glycoprotein efflux pump: how does it transport drugs?. J Membr Biol 1997;160:161-175

2. Bara, V. Digoxin overdose: clinical features and management: Vanessa Bara discusses the risk factors, antidotal therapy, and management of digoxin toxicity. Emerg Nurse 2001; 9:16-21

\section{Two Cases of Optic Neuritis Under Golimumab Therapy (Anti-TNFa) for Ankylosing Spondylitis Reveling Central Demyelinating Disorders}

\author{
C. Maucorps ${ }^{1}$, V. Chawadronow ${ }^{2}$, P. Branger ${ }^{2}$, N. Derache ${ }^{2}$, \\ S. Fedrizzi ${ }^{1}$, G. Defer ${ }^{2}$
}

${ }^{1}$ Department of Pharmacology, CHU de la Côte de Nacre, Caen, France, ${ }^{2}$ Department of Neurology, CHU de la Côte de Nacre, Caen, France 
Background: Tumor necrosis factor antagonists (anti-TNFa) became these last years an important drug class in support of many inflammatory diseases such as rheumatoid arthritis, ankylosing spondylitis (AS), disease inflammatory of the intestine or psoriasis. Despite effectiveness of these drugs, they could favor peripheral and central nervous system disorders. Aim: To present central demyelinating disorders probably linked to golimumab therapy.

Methods: We present two cases of pharmacovigilance reported spontaneously in our regional center concerning two optical neuritis potentially attributable to golimumab. A follow-up during several months was led in front of those clinically isolated syndrome (CIS) because of a high risk of developing multiple sclerosis (MS).

Results: The first patient is a 50-year-old male with a medical history marked by a diagnosis of AS in 2002, successively treated by adalimumab (2009), etanercept (2010) and then golimumab. After five years of treatment, he presented a bilateral optic neuritis. Brain magnetic resonance imaging (MRI) showed right optical, right orbito-frontal, left pontine and left corpus callosum T2 hyperintensities and oligoclonal bands were present in cerebrospinal fluid (CSF). Golimumab was withdrawn and after intravenous methylprednisolone bolus and plasma exchanges, visual acuity recovered. The second patient is a 31 -year-old female with a history of AS treated by adalimumab since 2012 and stopped by herself in 2014 in reason of pregnancy. Golimumab was introduced at the end of 2016. Five days after her first injection, the patient presented a left optic neuritis. MRI showed more than 9 lesions (periventriculars, juxtacorticals and one in the pons) without gadolinium enhancement and oligoclonal bands were present in CSF. Anti-TNFa was withdrawn and intravenous methylprednisolone bolus was performed with a good recovery. These two patients were diagnosed as CIS with high risk for developing MS. No new clinical event and no new MRI lesion was found at 3-month follow-up.

Conclusion: In those two cases, the occurrence of central demyelinating disorders is probably linked to golimumab (based on Naranjo scale) and this cannot be excluded. Demyelinating lesions or a potential of unmasking demyelinating lesions are suggested by the use and the chronology of the events [1]. In such situation, patients have to declare and they should consult their doctors [2]. Clinicians have to keep this in mind to stop definitively Anti-TNFa therapy (even the underlying disease) until other scientific evidences proved to the contrary.

Further sources of information/References:

1. Kemanetzoglou, E, Andreadou E. CNS Demyelination with TNF- $\alpha$ Blockers. Curr Neurol and Neurosci Rep 2017; 17:36

2. Maillart, E, Papeix, C, Mellerio C, et al. Extensive and severe CNS demyelination associated with golimumab therapy. J Neurol 2016;263:1869-1871

\section{Do they all agree?-comparing expert signal assessment}

\section{B. Grundmark ${ }^{1,2}$, D. Sartori ${ }^{1}$, J. Ellenius ${ }^{1,3}$}

${ }^{1}$ WHO-Uppsala Monitoring Centre, Uppsala, Sweden, ${ }^{2}$ Department of Surgery, Uppsala University, Uppsala, Sweden, ${ }^{3}$ Department of Learning, Informatics, Management and Ethics, Karolinska Institutet, Stockholm, Sweden
Background: Despite available methods and guidance for causality assessment and signal management [1,2], in-depth analyses on manual signal assessment and prioritisation [3] are sparse.

Objective/Aim: The aim was to compare the primary signal assessment outcome by experienced MD assessors as part of a study aiming to describe in detail their individual methodological approach.

Methods: A list of 20 previously unassessed drug event combinations (DEC) were extracted from VigiBase, the WHO global database of individual case safety reports, using the statistical signal detection method vigiRank [4]. Substances and events could further appear only once in the list, and some well acknowledged DECs were excluded manually. Five experienced Uppsala Monitoring Centre MDs were provided with the DEC list with basic data on cases: de/re-challenges, number of reporting countries, outcome, disproportionality measure and the full case-series, including narratives. Over the course of $3 \mathrm{~h}$, using any information sources available to them, they were asked to classify as many DECs as possible into signals for subsequent in-depth assessment or non-signals. Within $24 \mathrm{~h}$, participants were interviewed regarding assessment outcomes and their detailed methodological approach. Interviews were recorded, transcribed verbatim and analysed using inductive thematic analysis (ongoing).

Results: The participants completed 46 DEC assessments (7-11 each) where 4 DECs were classified by 1-3 participants as signals for in-depth assessment, see table. In 5 DECs, participants noted "other issues" worth pursuing. Four participants spent their most effort on DEC \#1 despite early comments by all, of it not being worth pursuing due to the event term being unspecific and the nature of the indication. DECs were generally not dismissed as non-signals without screening narratives for possible new signal-worthy aspects. The DEC that the assessors spent the least time on differed: two assessors named the same DEC (\#6) where the ADR term was ultimately identified by all as disease spill-over [5], i.e. the indication incorrectly coded as an event. Examples of "other issues" proposed for analysis were: investigating related/wider ADR term and/or drug class for signal value, personal interest, and proposing to inform about important pharmacokinetic issue.

Conclusion: Concordance regarding signal/non-signal classification of DECs was relatively high. Non-concordant classifications were partly due to differences in depth of assessment between assessors. The in-depth analysis of cognitive strategies is ongoing with plans to enlarge and broaden the study sample.

\section{Further sources of information/References:}

1. Hill, AB. The Environment and Disease: Association or Causation? ProcR SocMed 1965; 58:295-300

2. Pacurariu, A, et al. SCOPE Work Package 5: Signal ManagementBest Practice Guidehttp://www.scopejointaction.eu/_assets/files/ WP5-Signal-Management-Best-Practice-Guide(5).pdf

3. Pacurariu AC Coloma PM, Gross-Martirosyan L, Sturkenboom MC, Straus SM. Decision making in drug safety-a literature review of criteria used to prioritize newly detected safety issues. Pharmacoepidemiol Drug Saf 2017;26:327-34

4. Caster O, Juhlin K, Watson S, Norén GN. Improved statistical signal detection in pharmacovigilance by combining multiple strength-of-evidence aspects in vigiRank. Drug Saf 2014;37:61728

5. Grundmark B, Holmberg L, Garmo H, Zethelius B. Reducing the noise in signal detection of adverse drug reactions by standardizing the background: a pilot study on analyses of proportional reporting ratios-by-therapeutic area. Eur J Clin Pharmacol. 2014;70:627-35 
Table of assessed DECs (as ATC5 - MedDRA PT) as classified by participants A, B, C, $D$ and $E$ into signals and non-signals with other issues noted.

\begin{tabular}{|c|c|c|c|}
\hline DEC & $\begin{array}{l}\text { Potential } \\
\text { signal }\end{array}$ & Non-signal & Other issue \\
\hline $\begin{array}{l}\# 1 \text { L02BX other anti-hormones - } \\
\text { General physical health } \\
\text { deterioration }\end{array}$ & $\mathrm{D}, \mathrm{E}$ & $A, B, C$ & - \\
\hline $\begin{array}{l}\text { \#2N06AX Other antidepressants - } \\
\text { Hallucination }\end{array}$ & - & $A, B, C, D, E$ & - \\
\hline $\begin{array}{l}\# 3 \\
\text { C01BG Anti-arrhythmic Class III - } \\
\text { Cerebellar ataxia }\end{array}$ & - & $A, B, C, D, E$ & - \\
\hline $\begin{array}{l}\text { \#4 } \\
\text { L04AX Other } \\
\text { immunosuppressants - } \\
\text { Metastases to the liver }\end{array}$ & - & $A, B, C, D, E$ & D \\
\hline $\begin{array}{l}\text { \#5 } \\
\text { J07AN Vaccines against } \\
\text { tuberculosis - Pneumonitis }\end{array}$ & $\mathrm{D}^{*}$ & $A, B, C, E$ & $\mathrm{~B}^{*}$ \\
\hline $\begin{array}{l}\text { \#6 } \\
\text { L01XA Platinum compounds - } \\
\text { NSCLC }\end{array}$ & - & $A, B, C, D, E$ & - \\
\hline $\begin{array}{l}\# 7 \\
\text { N02AA Opiates - Urinary } \\
\text { Retention }\end{array}$ & - & $A, B, C, D, E$ & B \\
\hline $\begin{array}{l}\text { \#8 } \\
\text { L04AA Selective } \\
\text { immunosuppressants - Cervical } \\
\text { dysplasia }\end{array}$ & $C, D, E$ & A & - \\
\hline $\begin{array}{l}\text { \#9 } \\
\text { C03CA Sulphonamides - SIADH }\end{array}$ & - & $A, C, D$ & $A$ \\
\hline $\begin{array}{l}\# 10 \\
\text { A10BB Sulphonylureas - } \\
\text { Pancytopenia }\end{array}$ & C & $A, D$ & - \\
\hline
\end{tabular}

$\mathrm{DEC}=$ drug event combination

$\mathrm{NSCLC}=$ non-small-cell lung cancer

SIADH = syndrome of inappropriate $A D H$ secretion

* did not check or identify in labelling

\section{IntenSive Monitoring Program of TeratOgen and Non-Teratogen Risk of Drugs During Pregnancy: the STORK Project}

M. Tuccori ${ }^{1}$, I. Convertino ${ }^{2}$, M. Galiulo ${ }^{2}$, A. Capogrosso

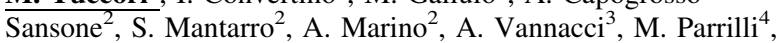
T. Corona ${ }^{5}$, C. Blandizzi ${ }^{1,2}$

${ }^{1}$ Unit of Adverse Drug Reactions Monitoring, University Hospital of Pisa, Pisa, Italy, ${ }^{2}$ Division of Pharmacology and Pharmacovigilance, Department of Clinical and Experimental Medicine, University of Pisa, Pisa, Italy, ${ }^{3}$ Department of Neurosciences, Psychology, Drug Research and Child Health, Section of Pharmacology and Toxicology, University of Florence, Florence, Italy, ${ }^{4}$ Tuscan Regional Centre of Pharmacovigilance, Pharmacy Department, Health District of Florence, Florence, Italy,

${ }^{5}$ Tuscan Region, Section of drug policies and appropriateness,

Florence, Italy
Introduction: Ethical issues limit pregnant women enrolment in clinical trials [1]. Observational studies may highlight neonates risk following drug exposure in pregnancy [2-4]. However, complicated studies design and related methodological shortcomings may limit results reliability. Consequently, great uncertainty surrounds the use of drugs during pregnancy.

Aim: To provide information to clinicians and women in Tuscany on drug exposure during pregnancy; to identify the most concerning drugs for pregnancy; and to evaluate the occurrence of adverse drug events in neonates.

Methods: The Stork project, carried out from 2012 to 2016, provided counselling to Tuscan clinicians and citizens, with the activation of the Teratologic Counselling Centre at Pisa University. Phone requests were recorded in data collection forms (demographic details, background information, call motivations) and answers were provided based on a priority flow-chart. The counselling provided a detailed report. When customers accepted to enter into the monitoring, an informed consent was requested for telephonic follow-up.

Results: Overall, 94 informal and 1 formal counselling were recorded and over-the-counter medications, for seasonal or pregnancy complications, were mostly involved. Inadequate information $(62.11 \%)$, second opinion $(18.95 \%)$, conflicting information $(13.68 \%)$, worrying symptoms $(4.21 \%)$ and information overload $(1.05 \%)$ were the calling motivations. Metoclopramide, amoxicillin, acyclovir, ibuprofen, paracetamol, dexchlorpheniramine, loratadine, dextromethorphan and antacids were the most enquired drugs. For the first trimester gestation, 43 calls, concerning inadequate information, were recorded on the following anatomical therapeutic chemical classifications: NO2B (other analgesics and antipyretics), R06A (systemic antihistamines), R05D (cough suppressants), M01A (antiinflammatories and anti-rheumatics), A02A (antacids). For the second trimester, 37 informal counselling, for inadequate information and second opinion, were recorded on: NO2B, R06A, J01C (penicillins), J05A (antivirals). For the third trimester, 14 calls, for inadequate and conflicting information, involved: R06A, A02A, N02B. In 71 cases the drug intake occurred actually. The $12 \%$ of these $(n=9)$ did not consent follow up, while the $87 \%(n=62)$ accepted, but only $14 \%(n=10)$ answered questionnaire. All 10 followed-up women delivered healthy neonates. One formal counselling was requested by a clinician at Pisa University Hospital, about the possible role of drugs taken by the mother (acyclovir, beclomethasone, ambroxol, miconazole, betamethasone, rubella vaccine) and the new-born death, occurred after delivery for respiratory distress. In the final report, no correlation was identified.

Discussion: This real-world analysis point to need for information on drug use during pregnancy.

Conclusion: Routine consultations could address physicians and patients to a safer and more aware approach of drug use during pregnancy. Further sources of information/References

1. Frew PM, Saint-Victor DS, Isaacs MB, Kim S, Swamy GK, Sheffield JS, et al. Recruitment and retention of pregnant women into clinical research trials: an overview of challenges, facilitators, and best practices. Clin Infect Dis2014;59 (Suppl 7):400-7

2. Schaefer C, Peters PWJ, Miller RK. Drugs During Pregnancyand Lactation: Treatment Options and Risk Assessment. Amsterdam: Academic Press. 2015: 1-918

3. Hameen-Anttila K, Jyrkka J, Enlund H, Nordeng H, Lupattelli A, Kokki E. Medicines information needs during pregnancy: a multinational comparison. BMJ Open. 2013; 3:e02594

4. Lupattelli A, Spigset O, Twigg MJ, Zagorodnikova K, Mardby AC, Moretti ME, et al. Medication use in pregnancy: a cross-sectional, multinational web-based study. BMJ Open. 2014; 4:e004365 


\section{What do patients know about Yellow Cards?}

\author{
$\underline{\text { A. Adams }}{ }^{1}$, C. Cassidy ${ }^{2}$, A. Thomas ${ }^{1}$, E. Carey ${ }^{1}$ \\ ${ }^{1} Y C C$ Wales, University Hospital Of Wales, UK, ${ }^{2}$ School \\ of Pharmacy, Cardiff University, Cardiff, UK
}

Introduction: An adverse drug reaction (ADR) is an unwanted or harmful reaction which occurs after administration of a drug or drugs and is suspected or known to be due to the $\operatorname{drug}(\mathrm{s})^{1}$. Since 2005 , the public have been able to directly report an ADR through the Medicines and Healthcare products Regulatory Agency's (MHRA) Yellow Card Scheme (YCS). In 2014/15 patients were responsible for 147 reports sent to the Yellow Card Centre in Wales. Evaluation of existing patient reporting schemes is scarce: while some countries have reported on the numbers and nature of reports, none appear to have reported on patient experience ${ }^{2}$.

Aim: To evaluate patients' knowledge and participation in the Yellow Card Scheme

Method: Purposive sampling was used to target those members of the public most at risk of having an ADR i.e. the elderly, those with a long term illness and those in poverty ${ }^{2}$. Census data was used to locate the electoral wards of Cardiff that had highest and lowest density of each risk group and then questionnaires were hand-delivered to approximately 66 randomly selected houses in each electoral ward $(n=400)$. Data were entered into SPSS ${ }^{\circledR}$ for descriptive analysis.

Results: There was a $14 \%$ response rate $(n=55)$, evenly distributed between gender and those with / without a limiting long term illness. The mean age of respondents was 50 years. Two participants were qualified health care professionals, one being a Pharmacist. Thirty-one participants (57\%) had heard the term ADR. Twenty eight participants $(50 \%)$ had experienced an ADR themselves however, only $4 \%(\mathrm{n}=2)$ of these participants had reported their ADR through the Yellow Card Scheme (YCS). Most reactions were to medicines prescribed by a doctor $(71 \%, \mathrm{n}=20)$ and most respondents $(64 \%, \mathrm{n}=18)$ reported the reaction to their doctor. $96 \%(n=53)$ of all respondents said if they were to experience an ADR in the future they would report it to their doctor; just $15 \%(\mathrm{n}=8)$ said they would report an ADR to the MHRA, although $67 \%(\mathrm{n}=37)$ said they would be comfortable reporting through the YCS. Only 16\% $(n=9)$ had previously heard of the YCS, mostly $(n=6)$ via the internet.. Over half of respondents $(56 \%$, $\mathrm{n}=31)$ requested to learn more about the YCS; online $(\mathrm{n}=20)$ or social media $(n=8)$ being the most preferred options.

Conclusion: The public needs more information regarding direct patient ADR reporting through the Yellow Card Scheme to help improve their engagement with pharmacovigilance.

Adverse drug reaction reporting should be promoted and encouraged through accessible and acceptable up to date methods such as social media, online and through smart phone apps.

Further sources of information/References

1. NICE. Adverse Drug Reactions. NICE; 2012 [accessed 10th November]. Available from: https://cks.nice.org.uk/adversedrug-reactions

2. Blenkinsopp A, Wilkie $\mathrm{P}$, Wang $\mathrm{M}$, Routledge PA. Patient reporting of suspected adverse drug reactions: a review of published literature and international experience. $\mathrm{Br} \mathrm{J}$ Clin Pharmacol 2007;63:148-56
101 Utilisation and Safety of Deferasirox (Exjade ${ }^{\circledR}$ ) in Older Adults: Results from an Observational Cohort Study in England

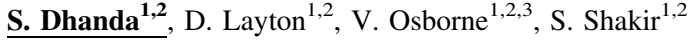 \\ ${ }^{1}$ Drug Safety Research Unit, Southampton, UK, ${ }^{2}$ University \\ of Portsmouth, Portsmouth, UK, ${ }^{3}$ Department of Epidemiology, \\ University of Florida, USA
}

Background: A UK study examined the safety and use of deferasirox prescribed in primary care in England. Deferasirox is an oral iron chelating agent indicated for treating chronic iron overload from repeated blood transfusions in patients with beta thalassaemia major and chelation therapy in other non-transfusion-dependent thalassaemias. Whilst dosing recommendations for elderly patients ( $\geq 65$ years) are the same as for younger age groups ( 2 years+), research has shown higher frequency of adverse reactions in the former. Closer monitoring for adverse events is recommended for this patient subgroup.

Objective/aim: A supplementary analysis to examine the utilisation and safety of deferasirox in patients aged $\geq 65$ years.

Methods: An observational cohort study. Patients identified from dispensed prescriptions of deferasirox Sept 06-Sept 14. Outcome data collected from prescribing general practitioners via questionnaires sent $\geq 6$ months after 1 st dispensed prescription. Summary descriptive statistics calculated; $\%$ denominator $\geq 65$ year subgroup, unless specified.

Results: Evaluable cohort $=122$ patients (median age 23 years (IQR $11-61)$ ). Twenty-seven patients aged $\geq 65$ years ( $22.1 \%$ cohort); 17 male (63.0\%). Starting dose specified for 12 patients $\geq 65 \mathrm{yrs}$ ( $44.4 \%$ ); 8 initiated on $10 \mathrm{mg} / \mathrm{kg} / \mathrm{day}$ and 4 patients on $20 \mathrm{mg} / \mathrm{kg} /$ day. Frequent reasons for prescribing include myelodysplastic syndrome $(\mathrm{n}=13)$, iron overload $(\mathrm{n}=3)$ and aplastic anaemia $(n=2)$. Majority of patients had treatment initiated by a specialist $(\mathrm{n}=22,81.5 \%)$. Serum creatinine ( $\mathrm{SCr}$ ) was reported for 6 patients $\geq 65$ years $(22.2 \%$ ) prior to starting; 3 were $>$ reference range (female $>90$ $\mu \mathrm{mol} / \mathrm{L} ;$ male $>110 \mu \mathrm{mol} / \mathrm{L})$. SCr was also reported for 6 patients after starting treatment, exceeding the reference in 3 female patients $(66-75$ years, $\mathrm{SCr}$ 96-116 $\mu \mathrm{mol} / \mathrm{L}$; baseline (bl) unknown) and 1 male patient (78 years, $\mathrm{SCr} 137$ $\mu \mathrm{mol} / \mathrm{L} ; \mathrm{bl}$ unknown). There was also an additional report of an increased $\mathrm{SCr}$ in a 74 year old female patient without prior renal disease ( $\mathrm{SCr} 135 \mu \mathrm{mol} / \mathrm{L}$; bl $\mathrm{SCr} 69 \mu \mathrm{mol} / \mathrm{L}$ ). A positive de-challenge was observed and the event was reported as a suspected adverse reaction to deferasirox.

Conclusions: These results show that deferasirox is being prescribed in older adults in England, with limited evidence of systematic monitoring in this patient subgroup. Despite recommendations for at least once monthly monitoring, missing data on renal measurements both prior to starting and during treatment were observed, in addition to events of raised SCr. Considering the small cohort of older adults, results should be put into context with other research evidence.

\section{Frail and elderly patient in oncology: increased drug toxicities?}

$\underline{\text { M. Abou Taam }}{ }^{1}$, J. Van Vynckt ${ }^{2}$, A. Provoost ${ }^{2}$, T. Trenque ${ }^{1,2}$

${ }^{1}$ Ea 3797, Faculté De Médecine, Université De Reims Champagne Ardenne, Reims Cedex, France, ${ }^{2}$ Regional Centre

of Pharmacovigilance and Pharmacoepidemiology, Reims Cedex, France 
Introduction: Specific patient are often excluded from oncology trials. Serious adverse drug reactions (ADRs) constitute major concerns in terms of both individual outcomes and public health expense. That is why observational studies are crucial to assess safety of antineoplastic in elderly and/or frail patients.

Aim: To identify risk factors associated with developing a serious ADR in oncology for frail or old patients.

Methods: We performed a retrospective and comparative study between patients treated by antineoplastic aged 65 and over versus younger patients. Sociodemographic information, performance status, Charlson's index, suspected drugs, off-label use, concomitant medications and ADRs data were analyzed. Data were collected from spontaneous reporting in a 1.3 M inhabitants regional pharmacovigilance centre, from January to December 2015. Descriptive statistics and logistic regression using SAS 9.4 were undertaken.

Results: We identified 167 patients (mean age $53.9 \pm 23.5$ years) who all underwent antineoplastic therapies. Elderly accounted $57 \%$ of the patients and experienced less serious ADRs $(\mathrm{p}<0.09)$. Among all the reported ADRs $(n=364), 30.4 \%$ referred to serious ones $(n=111)$. The majority of these serious ADRs were caused by targeted therapies $(n=16)$, pyrimidine analogue $(n=16)$ and nitrogen mustard $(n=15)$. In the logistic regression model assessing seriousness, the odds ratio (95\% confidence interval) for age was $0.37(0.15-0.91)$, for poor performance status (2-5) it was $1.36(0.49-3.75)$, for off label use it was 1.41 (0.54-3.67), for Charlson's Index 3-4 (ref. 0-2) it was $1.41(0.52-3.85$ ) and for targeted therapies it was $1.91(0.81-4.5)$. Polypharmacy was not associated with an increased risk of experiencing a serious ADR (1.1 (0.9-1.27)).

Conclusions: This study has not identified factors that contributed to developing serious ADRs. Both performance and comorbidity status were not associated with increased ADRs seriousness. A contrario, age was associated with decreased serious ADRs. The reasons are unclear: selection bias? undertreatment? Larger studies are required to support and understand those results.

\section{Oncogeriatric pharmacovigilance: a review of observational data}

\author{
M. Abou Taam ${ }^{1}$, T. Trenque ${ }^{1,2}$ \\ ${ }^{1}$ Ea 3797, Faculté De Médecine, Université De Reims Champagne \\ Ardenne, Reims Cedex, France, ${ }^{2}$ Regional Centre \\ of Pharmacovigilance and Pharmacoepidemiology, Reims Cedex, \\ France
}

Introduction: Specific patient are often excluded from oncology trials. Elderly should sometimes benefit from the antineoplastic. That is why observational studies are crucial to assess their safety.

Aim: To review all observational studies interested in oncogeriatric drug safety.

Methods: We systematically searched in Medline, until 30 june 2016, all observational studies, involving elderly and cancer agents, in english or french. Case series, case reports and articles that did not mention safety data were excluded. The primary outcome was the risk factors of ADRs in the elderly.

Results: The review included 145 of 38111 identified studies involving 183439 patients older than 65 years old. They were all treated with antineoplastic. They included 5905 older than 80 . Conflict of interest was reported in $43 \%$. Publication date was later than 2010 in $88 \%$. USA, Italy and Japan were the most important publishers (55\%). Studies were often retrospective $79 \%$ ). They were less frequently multicentre $(41 \%)$, comparative (10\%) or with younger counterparts (2\%). Safety data was a single primary outcome in only $14 \%$. It was an associated primary outcome in $6 \%$ and a secondary outcome in $8 \%$. The old age threshold was 70 in $44 \%, 65$ in $30 \%, 75$ in $14 \%$ and 80 in $4 \%$. Platines, targeted therapies, fluroruracil and taxanes were mainly studied. No immunotherapies were studied in those observational studies. Colorectal, lung, breast and pancreas cancer were the main indications. Comorbidity was a toxicity risk factor in 5\%. It was not in $6 \%$ and not assessed in remaining articles. In one study, bad performance status increased the toxicity. Age was associated with increased toxicity whatever the ADRs in $4 \%$, with specific ADRs in $17 \%$. They were no association in $15 \%$ and no assessment in $64 \%$. Grade I and II ADRs were mainly not collected. Drug discontinuations were assessed in only $26 \%$. Treatment-related hospitalizations were often not considered (94\%). Treatment-related death was mentioned in only $25 \%$.

Conclusions: Most of the published observational studies did not give enough importance to safety data. Instead of independent, prospective and comparative study, most of them were poor-quality designed. They often concluded about safety without having it in primary or secondary outcomes. High quality safety data should be mandatory to help elderly in making well-informed decision. This study has not clearly identified risk factors for ADRs. Further prospective and comparative studies are warranted to evaluate the drug safety in oncogeriatry.

\section{Safety and availability of codeine in France}

\author{
Abou Taam ${ }^{1}$, T. Trenque ${ }^{1,2}$ \\ ${ }^{1}$ Ea 3797, Faculté De Médecine, Université De Reims Champagne \\ Ardenne, Reims Cedex, France, ${ }^{2}$ Regional Centre \\ of Pharmacovigilance and Pharmacoepidemiology, Reims Cedex, \\ France
}

Introduction: Codeine, or methylmorphine, is a weak opiate used generally as painkillers or as antitussive agents. It is highly likely that there is a considerable degree of exposure to the high iatrogenic risk that the use of codeine-containing drugs incurs. The purpose of this study was to evaluate regional safety data regarding codeine use in a retrospective study spanning a 30 -year period. Secondarily, we also describe the various codeinecontaining medications available in France, as well as their compliance with current legislation.

Methods: This is a retrospective study including all codeine-related toxicities notified to our regional center of pharmacovigilance. A review of all codeine-containing medications authorized for sale in France was also performed.

Results: Over the 30 year period studied, 166 adverse effects occurred in 97 patients taking codeine. Among the 97 reports, $76 \%$ were serious, $20 \%$ voluntary overdose, $5 \%$ psychological addiction, $2 \%$ drug abusers and $2 \%$ drug withdrawal. $76 \%$ of the suspected codeine-containing drugs were unlisted drugs (OTC). In France, codeine is currently marketed in the form of 44 medications. Among these 44 medications, $64 \%$ are available OTC, $41 \%$ for cough.

Conclusion: Considering the numerous unlisted medication containing codeine and a probably high underreporting, the rate and the pattern of serious toxicities are troublesome. This study evidences also a high rate of misuse. This would imply that this drug should be available on prescription only. Physicians, pharmacists and patients need to be made aware of the potential dangers. Better tools for preventing iatrogenic complications need also to be developed and implemented. 


\section{Cerebral Pseudoatrophy, Dementia and Valproate}

\section{Abou Taam ${ }^{1}$}

${ }^{1}$ Ea 3797, Faculté De Médecine, Université De Reims Champagne Ardenne, Reims Cedex, France

Background: Valproate and its salts are widely used for epilepsy and psychiatric conditions. When their usefulness is well-established, there is insufficient awareness and knowledge about some neurological toxicity like dementia and brain scan abnormalities.

Objective/Aim: We investigated a dementia and pseudocerebral atrophy possibly induced by valproate and it salts by performing a systematic review of published case reports on this subject, a review of the World Health Organization VigiAccess database and of the European EudraVigilance database.

Results: The research found a total of 33 cases linking valproate and its salts to dementia in the literature. This dementia is sometimes described without encephalopathy or other neurological disorders. It occurs whatever the age and whatever the indication. We retrieved also 7 cases of pseudocerebral atrophy with dramatical resolution after valproate discontinuation. 139 cases of dementia related to valproate/valpromide were retrieved from the VigiAccess database (updated to April 2017), and 115 reports were found in EudraVigilance database (updated to April 2017) with $30 \%$ recovered patient. Concerning brain scan abnormal or cerebral atrophy, 3 cases were reported in EudraVigilance and 7 in Vigiaccess.

Discussion/Conclusion: An early withdrawn of valproate could be useful to obtain a complete remission of this adverse drug reaction and special attention should be particularly devoted to long-term therapy, to hypoalbuminemia [1], to kidney or hepatic impaired or elderly patients. Physician should be also aware about the neurological toxicity of other medications to avoid to labeled by mistake elderly patients as insane [2]. Some event-related potential (ERP) as N270 should be useful to detect minor cognitive impairment [3]. The Canadian Consensus Conference on the Diagnosis and Treatment of Dementia 2012 did not recommend the use of Valproate in dementia [4]. It should be useful to contra-indicated those drugs in dementia and to add in the SmPC cerebral pseudoatrophy as adverse effect.

Further sources of information/References

1. Sleegers MJM, Beutler JJ, Hardon WJ, Berden JHM, Verhave JC, Conemans JMH, et al. Reversible rapidly progressive dementia with parkinsonism induced by valproate in a patient with systemic lupus erythematosus. J Am Geriatr Soc 2010;58:799-801

2. Favrelière S, Lafay-Chebassier C, Alkhidir F, Merlet I, Pérault Pochat M-C. [Drug-induced dementia: a case/non-case study in the French Pharmacovigilance database]. Thérapie 2007;62:507-11

3. Sun W, Wang W, Wu X, Wang Y. Antiepileptic drugs and the significance of event-related potentials. J Clin Neurophysiol Off Publ Am Electroencephalogr Soc 2007;24:271-6

4. Herrmann N, Lanctôt KL, Hogan DB. Pharmacological recommendations for the symptomatic treatment of dementia: the Canadian Consensus Conference on the Diagnosis and Treatment of Dementia 2012. Alzheimers Res Ther 2013 8;5(Suppl 1):S5

\section{Impact of Serious Adverse Drug Reactions on the Quality of Life of Patients on HAART, in UMTH Maiduguri, North-East Nigeria}

\author{
P. Bassi ${ }^{1}$ \\ ${ }^{1}$ University of Abuja, Abuja, Nigeria
}

Background: Considerable progress has been made in providing access to antiretroviral therapy with more people receiving antiretroviral therapy in low- and middle-income countries [1]. However, the effectiveness of treatment programs, risks being compromised by problems related to toxicity [2]. The presence of HIV/AIDS as well as the symptoms and complications associated with HIV/AIDS treatment such as serious adverse drug reaction, may have a negative effect on the quality of life (QOL)of people living with HIV/AIDS [3]. This study, evaluated the impact of Serious ADR on the quality of life of HIV/AIDs patients on HAART.

Methods: We conducted a prospective cohort study of naive adult HIV patients initiated on ART in our HIV Clinic from January 2006 to December 2010. Patients with and without serious ADRs during follow-up were compared to determine the impact of ADR on their QOL. A total cohort of 400 was administer questionnaires to and compare the QOL at the end of 48 months on ART. The WHOQOL-HIV BREF [4] was employed for measuring QOL of patients. Descriptive statistics, was used to summarize the score of the QOL. ANOVA test was used to examine the differences between average health related quality of life domain scores. Statistical significance was determined using the $95 \%$ confidence intervals with $P$. Value set at 0.05 .

Results: There were 373 respondents with mean overall age of 40.8 years (SD \pm 8.64$)$. More patients were female $(68.1 \%)$ compared to males $(31.9 \%)$. Majority of the patients $(93.3 \%)$ were within the reproductive age group, $52.8 \%$ were married, $22.8 \%$ were either single/divorced/separated, or cohabiting while $24.4 \%$ were widow/widower. And $53.4 \%$ patients had at least one serious ADR during the 48 months of follow-up. Patients who had experienced serious ADR had consistently and significantly lower mean QOL scores compared to patients who did not experience serious ADRs in the domains of psychological state of health $(62.5795 \% \mathrm{CI}$; 59.92-65.17 versus $65.97,95 \% \mathrm{CI}$; 64.01-67.82) $P=0.000$, social relationship in health $(46.9495 \% \mathrm{CI}$; $43.81-50.26$ versus $51.9795 \% \mathrm{CI}$; 48.60-55.50) $P=0.037$ and environments state of health $(56.9195 \% \mathrm{CI}$; $54.60-59.24$ versus $60.7795 \% \mathrm{CI} ; 58.30-63.06) P=0.028$ respectively. Conclusion: This study revealed that having experience of serious ADRs is associated with lower quality of life in health state domains, indicating how serious ADR has impact on QOL of HIV patients despite interventions such as counselling and the effectiveness of HAART.

Further sources of information/References

1. UNAIDS: AIDs By the Numbers; It is not over but it can be. Joint United Nations Programme on HIV/AIDS 2015

2. Edwards IR, Brielle C. Drug Safety. 2001; 22(6):416-423

3. Bakare N, Edwards IR, Stergachis A, Pal S, Holmes CB, et al. Global Pharmacovigilance for Antiretroviral Drugs: Overcoming Contrasting Priorities. PLoS Med 2011; 8: e1001054. 
4. World Health Organization Quality Of Life (WHOQOL) Group, 1994. Development of the WHOQOL: rationale and current status. Int J Ment Health 23:24-56

\section{Early Onset Vemurafenib-Cobimetinib Induced Drug Rash with Eosinophilia and Systemic Symptoms (DRESS) Syndrome: a Case Series}

D. Bourneau-Martin ${ }^{1}$, G. Veyrac ${ }^{2}$, C. Bernier ${ }^{3}$, L. Gaboriau ${ }^{4}$, J. Ponte-Astoul ${ }^{5}$, N. Paret ${ }^{6}$, C. Philibert ${ }^{7}$, B. Azzouz ${ }^{8}$, M. DaminPernik $^{9}$, H. Bagheri ${ }^{10}$, M. Blanche Valnet Rabier ${ }^{11}$, G. Drablier ${ }^{1}$, L. Lagarce ${ }^{1}, M$. Briet ${ }^{1}$

${ }^{1}$ Regional PharmacoVigilance Center, University Hospital of Angers, Angers, France, ${ }^{2}$ Clinical Pharmacology Department, Biology institute, University Hospital of Nantes, Nantes, France,

${ }^{3}$ Dermatology Department, University Hospital of Nantes, Nantes, France, ${ }^{4}$ Pharmacovigilance Regional Center, University Hospital of Lille, Lille, France, ${ }^{5}$ Regional Pharmacovigilance Center of Marseille Provence Corse, Pharmacovigilance and Clinical Pharmacology Department, Marseille, France, ${ }^{6}$ Regional PharmacoVigilance Center of Lyon, University Hospitals, Lyon, France, ${ }^{7}$ Pharmacovigilance Regional Center, Department of Médical Pharmacology and Toxicology, Montpellier University Hospital, Montpellier, France, ${ }^{8}$ Regional Center of Pharmacovigilance and Pharmacoepidemiology, Reims University Hospitals, Reims, France, ${ }^{9}$ Regional PharmacoVigilance Center, University Hospital of Saint Etienne, Saint Etienne, France,

${ }^{10}$ Clinical Pharmacology Department, Midi-Pyrenees

Pharmacovigilance, Pharmacoepidemiology and Drug Information Center, Toulouse University Hospital, Toulouse, France, ${ }^{11}$ Regional PharmacoVigilance Center, University Hospital of Besançon, Besançon, France

Background: Vemurafenib (a BRAF inhibitor) and cobimetinib (a MAPK inhibitor) are indicated for metastatic or unresectable melanoma with BRAF mutations. Drug induced DRESS syndrome often occurs within 2 to 6 weeks. However, several cases of vemurafenib-cobimetinib-induced DRESS syndromes were reported with a shorter delay.

Objective: The aim of our study was to characterize these DRESS syndromes.

Method: All cases of DRESS syndromes associated with vemurafenibcobimetinib registered in the French Pharmacovigilance were selected. Following data were analyzed: demographic data, occurrence delay, clinical signs, outcome, concomitant drugs and previous treatment lines. Results: A total of 15 cases ( 6 female and 9 male) were identified. The mean age of patients was 53 years [43-81]. Median onset delay of DRESS syndromes was 7 days [1-30]. Thirrteen DRESS syndromes occurred within less than 2 weeks. DRESS syndrome was diagnosed according to the RegiSCAR International Consensus Criteria between 3 and 8 (from possible to definite). Favourable outcome was noted in 14/15 cases with corticotherapy (7/15 cases), without recurrence. When data were known, it was the first-line treatment for 2 patients, the second for 3 patients, the third for 1 patient and the fourth for 2 patients. Previous lines involved were: interferon $(\mathrm{n}=3)$, dabrafenib $(\mathrm{n}=1)$, ipilimumab $(\mathrm{n}=2)$, nivolumab $(\mathrm{n}=3)$, pembrolizumab $(\mathrm{n}=2)$, and dabrafenib + trametinib $(\mathrm{n}=1)$.

Discussion/Conclusion: The onset delay of these cases of DRESS syndrome is shorter than those usually observed. In literature, 5 cases [1-4] of DRESS syndrome induced by vemurafenib alone were reported, of which 3 [1-2] occurred within a delay less than 2 weeks. Moreover, 2 patients had also been previously treated with anti-PD1. In the Global
Pharmacovigilance Database (Vigilyze ${ }^{\circledR}$ ), 19 cases have been recorded (no French cases): 14 involving vemurafenib, including 2 with a delay $\leq 2$ weeks (data known in 8 cases) and 5 with vemurafenib and cobimetinib, including 1 with a delay $\leq 2$ weeks (data known in 2 cases). To the best of our knowledge, there is no physiopathological mechanism explaining the short onset delay. This raises several questions: could cobimetinib play a role in the onset of DRESS syndrome and accelerate the first symptoms with a starter role? Or is the reduced onset delay caused by other lines of treatment or by a combination of several factors? To avoid delayed care, clinicians should be aware that vemurafenib and cobimetinib could induce a DRESS syndrome with a short delay.

Further sources of information/References

1. Johnson D,et al. Severe cutaneous and neurologic toxicity in melanoma patient during vemurafenib administration following anti PD-1 therapy. Cancer Immunol Res 2013; 1(6) :373-7

2. Munch M, et al. Early-onset vemurafenib-induced DRESS syndrome. Dermatology 2016; 232: 126-128

3. Gey A,et al. Severe cutaneous adverse reaction associated with vemurafenib DRESS, AGEP or overlap reaction? JEADV 2016; 30: 178-179

4. Wenk KS,et al. Vemurafenib-induced DRESS. JAMA Dermatology 2013; 149(10): 1242-1243

\section{Latency period of crying following vaccination with MMR vaccine. What do spontaneous AEFI- reports show?}

\author{
$\underline{\text { A. van Boekel }^{1}}$, L. Härmark ${ }^{1}$, E. van Puijenbroek ${ }^{1}$ \\ ${ }^{1}$ Pharmacovigilance Center Lareb, 's-Hertogenbosch, \\ the Netherlands
}

Background: Vaccination may lead to a variety of non-specific reactions that can be attributed to the (painful) injection or a local or systemic reaction. A frequently reported, non-specific reaction is crying. Crying after vaccination may arise directly in response to the injection, but can also result from general malaise, fever or pain. Usually crying occurs immediately, or on the first day following vaccination. For measles, mumps and rubella (MMR) vaccination crying due to malaise or physical discomfort can also occur after approximately 5 to 14 days [1,2], since it takes time for the live attenuated pathogens to replicate and establish an immune response.

Aim: To analyse the number of reports and latency period of crying after immunization with a live attenuated MMR vaccine.

Methods: Reports submitted to the Netherlands Pharmacovigilance Centre Lareb between 1 June 2010 and 5 February 2016 with the MedDRA Preferred Term 'crying' following immunization with an MMR vaccine were included. For all reported episodes the latency period was analysed.

Results: In total 327 reports of 'crying' were received in relation to vaccination with a MMR vaccine. In 297 of these reports the latency period could be calculated. In $29 \%(\mathrm{n}=85)$ crying occurred within $24 \mathrm{~h}$ after vaccination. In 30 of these cases an injection site reaction (swelling, redness, pain) was co-reported. In $71 \%$ of the reports $(n=212)$ the time to onset was more than $24 \mathrm{~h}$; in 149 cases crying occurred after 5 to 14 days, with a peak at day 7 .

Conclusion: Parents and health care professionals often consider crying shortly after vaccination as an expected and logical response to the injection. In respect to MMR vaccination, crying may also occur after 5 to 
14 days. This is consistent with the latency period of fever and malaise after vaccination with the MMR vaccine, caused by replication of the live attenuated pathogens in the vaccine. Since parents do not expect crying at this time point, it may be a cause of concern for them. Although one should stay alert for possible other causes for crying, health care professionals should inform parents that crying 5 to 14 days after vaccination with the MMR vaccine may be expected.

Further sources of information/References

1. Virtanen M, Peltola H, Paunio M, Heinonen OP. Day-to-day reactogenicity and the healthy vaccinee effect of measles-mumpsrubella vaccination. Pediatrics 2000;106:E62

2. Moorer N. Vraag uit de praktijk. Bijwerking of mazelen? Infectieziekten Bulletin 2011; 22: 203

\section{Hepatotoxicity associated with anti-tuberculosis drugs. A case series from hospitalized patients in Lima, Perú}

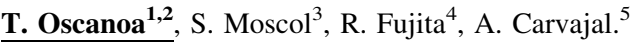

${ }^{1}$ Centro Investigación Seguridad Medicamentos. Facultad Medicina H. Universidad San Martin De Porres, Lima, Peru, ${ }^{2}$ Departamento de Medicina del Hospital Almenara, Lima, Peru, ${ }^{3}$ Pneumology Service of Almenara Hospital, Lima, Peru, ${ }^{4}$ Instituto de Biología y Genética Molecular, Facultad de Medicina de la Universidad de San Martin de Porres, Lima, Peru, ${ }^{5}$ Centro de Estudios sobre la Seguridad de los Medicamentos, Universidad de Valladolid, Valladolid, Spain

Introduction The incidence rate of tuberculosis (TB) in Peru is 119 per 100,000 inhabitants. The worldwide frequency of ADRs to anti-TB firstline drugs (isoniazid, rifampicin, pyrazinamide and ethambutol) ranges between 3.4 and 13\% [1]; among them, hepatotoxicity is considered as the most severe (Drug-induced liver injury or DILI).

Aim To know the clinical characteristics of liver injury secondary to antiTB treatment in hospitalized patients.

Methods Medical records of interest were reviewed. We chose those of patients hospitalized between January 2014 and December 2016, which met the following criteria: (1) older than 18 years, (2) diagnosis of tuberculosis by bacilloscopy and / or culture, (3) treatment with antituberculosis drugs with first-line drugs and iv) clinical diagnosis of -induced liver injury (DILI) secondary to anti-TB treatment. The RUCAM (Roussel Uclaf Causality Assessment Method) criteria was used for causality assessment.

Results During the two-year period of study, 507 cases of tuberculosis were hospitalized at Almenara Hospital in Lima, Peru. Out of those, 18 (3.6\%) cases of DILI due to anti-TB treatment were observed: $10(55.6 \%)$ associated with pyrazinamide (Z); 6 (33.3\%) and 2 (11.1\%) with isoniazid and rifampicin, respectively. The mean age was 42.0 years (range, 17-76 years); male $11(61.1 \%)$ and female $6(38.9 \%)$. The most frequent symptoms were nausea and vomiting $6(33.3 \%)$, jaundice $6(33.3 \%)$, rash and pruritus 5 (27.7\%). According to RUCAM criteria, 7 (38.8\%) were cholestatic, $5(27.7 \%)$ hepatocellular and $6(33.3 \%)$ mixed. The mean alkaline phosphatase was $316.8 \pm 151.2$ (range, $75-610 \mathrm{U} / \mathrm{L}$ ) and alanine aminotransferase was $295.9 \pm 288.3 \mathrm{U} / \mathrm{L}$ (range, 16-934 U / L).
The associated comorbidities were, HIV infection (2), diabetes mellitus (1) and liver cirrhosis (1).

Conclusions Pyrazinamide is probably the most common cause of liver injury induced by first-line anti-TB drugs. The usual form of presentation is cholestasic.

Further sources of information/References:

1. Tostmann A, Boeree MJ, Aarnoutse RE, de Lange WC, van der Ven AJ, Dekhuijzen R. Antituberculosis drug-induced hepatotoxicity: concise up-to-date review. J Gastroenterol Hepatol 2008;23:192-202

\section{State of play on the reporting of adverse reactions of direct oral anticoagulants by doctors in Rabat (MOROCCO) in 2016}

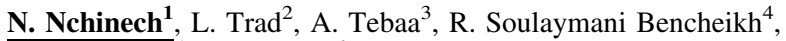
Y. Bousliman $^{5}$, S. Makram ${ }^{6}$

${ }^{1}$ Faculty of Medicine and Pharmacy-Mohamed V University, Pharmacy Department-Mohammed V Military Teaching Hospital, Rabat, Morocco, ${ }^{2}$ Faculty of Medicine and Pharmacy-Mohamed V University, Rabat, Morocco, ${ }^{3}$ Centre Anti Poison et de Pharmacovigilance du Maroc, Rabat, Morocco, ${ }^{4}$ Faculty of Medicine and Pharmacy-Mohamed V University, Centre Anti Poison et de Pharmacovigilance du Maroc, Rabat, Morocco, ${ }^{5}$ Faculty of Medicine and Pharmacy-Mohamed V University, Pharmacy DepartmentMohammed V Military Teaching Hospital,, Rabat, Morocco, ${ }^{6}$ Faculty of Medicine and Pharmacy-Mohamed V, Pharmacology Department, Rabat, Morocco

Introduction: The conventional anticoagulation by vitamin $\mathrm{K}$ antagonists (VKAs) used for decades despite its effectiveness has posed several problems. New oral anticoagulants designated under the name direct oral anticoagulants (DOACs) are appearing in the physician's armamentarium for several years and vest over clinical studies more and more indications. Under-reporting of adverse drug reactions (ADRs) is an important issue in pharmacovigilance. In this context, the Moroccan Ministry of Health issued the circular of January 4, 2016 making the reporting of ADRs mandatory by health professionals.

Aim: To Study the adherence of doctors to spontaneous reporting of ADRs of direct oral anticoagulants.

Méthods: This is a prospective observational study, survey type including 84 doctors, practicing in Rabat, in the public and private sectors. This study took place between March 3, 2016 and May 3, 2016. Physicians were interviewed through a questionnaire of 25 questions.

Results: The study participation rate was $59.52 \%$ (50 physicians). $32.6 \%$ of doctors report having had an accident related to the use of DOACs, regardless of its severity, $78.6 \%$ of whom reported that they had already experienced life-threatening haemorrhage requiring hospitalization. Concerning the actual reporting, 01 cardiologist $(7.69 \%)$ reported the side effect occurred at the CAPM. $80 \%$ believe it is important to report severe ADRs, but $44 \%$ are unaware of the need to report previously identified ADRs to clarify their impact. Physicians attribute underreporting to a lack of knowledge of the reporting system (84\%), lack of time (76\%), but also fear of blaming their professional responsibility $(82 \%)$. 
Conclusion: Our study shows an under-reporting of AEs related to the use of DOACs by physicians participating in the survey. So, it is necessary to raise awareness among health professionals about the importance of spontaneous reporting.

\section{Assessment of cancer patients' knowledge of the management of adverse reactions at the Mohammed V Rabat Military Instruction Hospital}

\author{
N. Nchinech' ${ }^{1}$, M. El Harrak ${ }^{2}$, Y. Tadlaoui ${ }^{3}$, A. Tebaa ${ }^{4}$, \\ R. Soulaymani Bencheikh ${ }^{5}$, Y. Bousliman ${ }^{6}$, S. Makram ${ }^{7}$
}

${ }^{1}$ Faculty of Medicine and Pharmacy-Mohamed V University, Pharmacy Department-Mohammed V Military Teaching Hospital, Rabat, Morocco, ${ }^{2}$ Faculty of Medicine and Pharmacy-Mohamed $V$ University, Rabat, Morocco, ${ }^{3}$ Faculty of Medicine and PharmacyMohamed V University, Pharmacy Department-Mohammed V Military Teaching Hospital, Rabat, Morocco, ${ }^{4}$ Centre Anti Poison et de Pharmacovigilance du Maroc, Rabat, Morocco, ${ }^{5}$ Centre Anti Poison et de Pharmacovigilance du Maroc, Rabat, Morocco, ${ }^{6}$ Faculty of Medicine and Pharmacy-Mohamed V, Pharmacology Department, Pharmacy Department-Mohammed V Military Teaching Hospital, Rabat, Morocco, ${ }^{7}$ Faculty of Medicine and Pharmacy-Mohamed V, Pharmacology Department, Rabat, Morocco

Introduction: In Morocco, cancer is the second leading cause of death after cardiovascular disease. Among anticancer drugs, cytotoxics are not selective and their action on healthy cells is the cause of adverse effects whose quantification, prevention and management must be an integral part of their care. In this context, knowledge of the adverse effects of the drugs administered as well as the spontaneous reporting by the patients themselves are an important component in the cancer control strategy.

Aim: To evaluate the knowledge on the management of adverse effects and pharmacovigilance of patients treated with anticancer drugs at the Military Instruction Hospital Mohammed V (HMIMV) in Rabat.

Material and Method: This is a prospective, observational study carried out at the The Functional Unit for the Management of Products with Special Status of the HMIMV pharmacy, involving a population of 132 cancer patients receiving anticancer agents, during a pharmaceutical interview through a questionnaire, while 52 days, covering the months of February and March 2016. Data were analyzed with SPSS statistics software version 13.0

Results: The study participation rate was $96.9 \%$. Mean age was $55 \pm 11.32$ years. The sex ratio was 0.61 (men/women). $90.2 \%$ or 119 of the patients surveyed had optimal therapeutic compliance, $9.8 \%$ or 13 have poor compliance, of which 4 patients have voluntary non-compliance. $57 \%$ of patients say they contact their doctors in the event of adverse events, $31 \%$ contact their pharmacists and $12 \%$ do nothing. 120 patients or $90.9 \%$ know the need to report adverse events to the doctor or pharmacist, compared with 9 patients (6.8\%). 103 patients, or $78 \%$, often do so. 125 patients $(94.7 \%)$ were unaware of the signs of infection with a medical device for administering injectable chemotherapy. 96.9\% (128 patients) wanted particularly to receive additional explanations of the side effects of their treatments.

Conclusion: This study showed that patients must be continually sensitized to these adverse effects so that they can be prevented and better understood. This led to the creation of a therapeutic education unit to compensate for the growing need for patient information and to emphasize the practicality of the spontaneous reporting and the interest to report.

\section{Pharmacovigilance requirements for Clinical trials in emerging Markets in Asia}

\author{
S. Miglani ${ }^{1}$ \\ ${ }^{1}$ Apcer Life Sciences, New Delhi, India
}

Background: Pharmacovigilance, being a very sensitive issue, demands a high degree of regulatory expertise especially in global clinical trials. There are numerous differences in the drug regulations within the different countries of Asia. This session will cover the important pharmacovigilance regulations and the various measures to manage the complexities associated with it.

Objective: The objective of the study was to understand the important differences in pharmacovigilance regulations for clinical trials in Asia and other developing countries, compare these regulatory environments to those in the US and EU, and explore measures to manage complexities. Method: A review of the regulatory and clinical trial PV systems literature was done. A comprehensive assessment of the safety reporting requirements in China, Japan, Korea, Taiwan, Singapore and some of the other countries in Asia was done to understand the differences and similarities amongst them.

Results: While the West has witnessed major developments in pharmacovigilance, not much has been observed in Asian countries. The study revealed that there is considerable heterogeneity and diversity in the regulatory requirements of the participating countries and a precise knowledge is necessary to streamline pharmacovigilance operations.

To exemplify, a local representative is quintessential in China, Japan, and Taiwan, while that is not the case in some of the other countries. Further, the translation of SUSARs to the local language is obligatory in some countries such Japan and South Korea; however, English version is still acceptable in many others. Differences also exist in the mode of submission of reports with different countries opting for manual submission/ in person submission or electronic submission. The variations are not limited to the above mentioned examples but extend across many other nuances in the modus operandi of pharmacovigilance.

Conclusion: Asia is the fastest growing pharmaceutical market in the world, providing substantial opportunities for drug development and marketing. Pharmacovigilance being a very sensitive issue demands a high degree of regulatory expertise especially in Global clinical trials. Harmonizing pharmacovigilance regulations in clinical trials has been a continuous challenge due to diverse geographical, cultural and clinical practices in this region. However, with more R\&D activities conducted in Asia, there is an important requirement to streamline pharmacovigilance processes. Extensive knowledge of the modus operandi of ethics committees plays a crucial role in achieving the highest standards in patient safety.

Further sources of information/References:

1. Analysis Report Identification and Clarification of the Differences in Regulatory Requirements between Asian Economies, Tokyo, Japan [Internet] 2015 Apr [cited 2016 May 26]. Available from: http://www.jpma.or.jp/english/apac/pdf/analysis_report2015.pdf

2. International Conference on Harmonization of Technical Requirements for Registration of Pharmaceuticals for Human Use. Clinical Safety Data Management: Definition and Standards for Expedited Reporting E2A. 1994. [cited 2016 May 26] Available from: https://www.ich.org/fileadmin/Public_Web_Site/ICH_Products/Guide lines/Efficacy/E2A/Step4/E2A_Guideline.pdf

3. Safety Reporting Requirements for Clinical Drug Trials. Regulatory Guideline, [Internet]. 2011 Jun [cited 2016 May 30] Available from: http://www.hsa.gov.sg/content/dam/HSA/HPRG/Clinical_Trials/ 
Clinical\%20Trials\%20Safety\%20Reporting\%20Guidelines_June\% 202011.pdf

4. Pharmaceutical Administration and Regulations in Japan. Regulatory Information Task Force. Japan Pharmaceutical Manufacturers Association. [Internet]. 2015. [cited 2016 May 30], Available from: http://www.jpma.or.jp/english/parj/whole.html

5. Korea Food and Drug Administration Notification No. 1999-67. Guideline for Korean Good Clinical Practice. [Internet]. 2000. [cited 2016 May 30], Available from: http://www.google.co.in/url?sa=t\&rct $=\mathrm{j} \& \mathrm{q}=\&$ esrc $=$ s\&source $=$ web $\& \mathrm{~cd}=1 \& \mathrm{ved}=0$ ahUKEwiFu8PXZLNAh VEipQKHbkUDGQQFggbMAA\&url=http\%3A\%2F\%2Fwww.gcphel pdesk.com\%2Fknowledgebase $\% 2$ Fitem $\% 2$ Fdownload $\% 2 F 43 \& u s g=$ AFQjCNFrcX8shH93W4aR-fkSeFIxxldLPQ

6. Regulations for Implementation of the Drug Administration Law of the People's Republic of China. [Internet]. 2002. [cited 2016 May 30], Available from: http://eng.sfda.gov.cn/WS03/CL0767/61640.html

\section{A description and comparison of the adverse drug reaction profiles in people living with HIV administered antitubercular and/or antiretroviral medications}

\section{J. Tatenda Masuka ${ }^{1}$, P. Chipangura ${ }^{1}$, L. Gwaza ${ }^{1}$, P. Nyambayo ${ }^{1}$, G. Mahlangu}

\section{${ }^{1}$ Medicines Control Authority of Zimbabwe, Harare, Zimbabwe}

Introduction: A few studies describe the adverse drug events (AEs) in TB/HIV co-infected patients simultaneously receiving antiretroviral and antitubercular medicines in resource-limited countries [1-3]. Studies in developed countries show an overlap and increase in AEs in this population, but there is limited characterization in patients with advanced HIV and TB with comorbid poor nutritional status $[1,3]$.

Objectives: To describe and compare the adverse drug reaction profile between patients on antiretroviral drugs only (HAART) to those co-administered antiretrovirals and isoniazid (HHAART) or antiretroviral and antitubercular drugs (ATTHAART) in an African setting.

Methods: We performed a retrospective review of Individual Case Safety Reports (ICSRs) submitted to the Zimbabwean national pharmacovigilance centre from September 2012 to August 2016. All reports gathered through the targeted spontaneous reporting of antiretroviral and/or antitubercular medicines considered certain, probable or possible were considered for analysis [4].

Results: A total of 1114 individual case safety reports were included in the analysis. Seventy-six percent of the reports were from the HAART only group, with $12 \%$ and $6 \%$ for the HHAART and ATTHAART groups respectively. More reports were observed in the HHART and ATTHAART groups for hepatic disorders $(\mathrm{p}<0.0001)$ and peripheral neuropathy ( $\mathrm{p}=0.0013$ ) compared to the HAART only group. Rash and Stevens-Johnson syndrome occurred more frequently in the HAART only group compared to the other treatment groups $\mathrm{p}=0.0119$ and $\mathrm{p}=0.0003$ respectively.

Conclusion: Co-administration of antiretroviral and antitubercular medicines increases the frequency of drug induced liver injury and peripheral neuropathy in an African resource-limited setting.

Further sources of information/References

1. McIlleron H, Meintjes G, Burman WJ, Maartens G. Complications of antiretroviral therapy in patients with tuberculosis: drug interactions, toxicity, and immune reconstitution inflammatory syndrome. J Infect Dis 2007; 196 (Suppl 1): S63-75
2. Sadiq S, Khajuria V, Tandon VR, Mahajan A, Singh JB. Adverse Drug Reaction Profile in Patients on Anti-tubercular Treatment Alone and in Combination with Highly Active Antiretroviral Therapy. J Clin Diagn Res JCDR. 2015; 9: FC01-04

3. Manosuthi W, Wiboonchutikul S, Sungkanuparph S. Integrated therapy for HIV and tuberculosis. AIDS Res Ther 2016 May 12; 13: 22

3. Pal SN, Duncombe C, Falzon D, Olsson S. WHO strategy for collecting safety data in public health programmes: complementing spontaneous reporting systems. Drug Saf 2013; 36: 75-81

Table 1: Comparison of ADR/ICSR rates in respective treatment group

\begin{tabular}{|c|c|c|c|c|c|c|}
\hline \multirow[t]{2}{*}{ ADR (p value) } & \multicolumn{2}{|l|}{ HAART } & \multicolumn{2}{|c|}{ HHAART } & \multicolumn{2}{|c|}{ ATTHAART } \\
\hline & $n / N$ & $\%$ & $n / N$ & $\%$ & $n / N$ & $\%$ \\
\hline Rash (0.0119) & $259 / 882$ & 29.37 & $47 / 132$ & 35.61 & $9 / 62$ & 14.52 \\
\hline $\operatorname{DILI}(<.0001)$ & $44 / 882$ & 4.99 & $45 / 132$ & 34.09 & $22 / 62$ & 35.48 \\
\hline $\begin{array}{l}\text { Peripheral } \\
\text { neuropathy }(0.0013)\end{array}$ & $76 / 882$ & 8.62 & $4 / 132$ & 3.03 & $12 / 62$ & 19.35 \\
\hline $\begin{array}{l}\text { Renal impairment } \\
(0.9900)\end{array}$ & $65 / 882$ & 7.37 & $10 / 132$ & 7.58 & $5 / 62$ & 8.06 \\
\hline SJS (0.0003) & $130 / 882$ & 14.74 & $4 / 132$ & 3.03 & $1 / 62$ & 1.61 \\
\hline $\begin{array}{l}\text { Gynaecomastia } \\
\text { (0.8869) }\end{array}$ & $161 / 882$ & 18.25 & $2 / 132$ & 1.51 & 0 & 0 \\
\hline Anaemia (0.8869) & $21 / 882$ & 2.38 & $2 / 132$ & 1.52 & $1 / 62$ & 1.61 \\
\hline Lipodystrophy & $39 / 882$ & 4.42 & 0 & 0 & 0 & 0 \\
\hline Psychosis (0.1854) & $7 / 882$ & 0.79 & $6 / 132$ & 4.55 & $1 / 62$ & 1.61 \\
\hline Dizziness (0.7906) & $46 / 882$ & 5.22 & $6 / 132$ & 4.55 & $2 / 62$ & 3.23 \\
\hline
\end{tabular}

$\mathrm{N}=$ total number of ICSRs within the group and $\mathrm{n}=$ total number of ICSRs in the treatment group with the ADR

\section{Disease-Modifying Antirheumatic Drugs (DMARDs) Utilisation in Autoimmune Rheumatic Diseases}

\author{
L. Anghel ${ }^{1}$, D. Leucuţa ${ }^{2}$, A. Farcaş ${ }^{3}$, R. Oprean ${ }^{1}$ \\ ${ }^{1}$ Analytical chemistry and instrumental analysis, Faculty \\ of Pharmacy, University of Medicine and Pharmacy Cluj-Napoca, \\ Romania, ${ }^{2}$ Medical Informatics and Biostatistics, Faculty \\ of Medicine, University of Medicine and Pharmacy, Cluj-Napoca, \\ Romania, ${ }^{3}$ Drug Information Research Centre, Faculty of Pharmacy, \\ University of Medicine and Pharmacy, Cluj-Napoca, Romania
}

Introduction: Disease-modifying antirheumatic drugs (DMARDs) remain the first choice of treatment in autoimmune rheumatic diseases. Data regarding the extent in which they are used can give essential information that is needed to achieve a better management of these diseases [1-3]. Since reports in this particular area are scarce in Romania, this study will add relevant information to this domain.

Objective: To evaluate DMARDs utilisation in a real life setting.

Methods: We conducted a retrospective observational study using a pharmacy claim database. All prescriptions from the database starting $1^{\text {st }}$ of January 2011 to $31^{\text {st }}$ of December 2014 were analysed. A number of 7 DMARDs (azathioprine, sulfasalazine, hydroxychloroquine, leflunomide, methotrexate, methylprednisolone and ciclosporin) are prescribed by doctors being fully reimbursed by the National Insurance Health System in Romania. By using the specific coding for rheumatoid arthritis (RA), ankylosing spondylitis (AS), systemic lupus erythematosus (LES) and psoriatic arthritis (PsA) and the above mentioned compounds we 
determined the prevalence of each DMARD used as single and combined therapy.

Results: Out of a total number of prescriptions $(n=486,700)$ of the 4 years study timeframe, a number of $5375(1.1 \%)$ prescriptions were for DMARDs in 1085 patients. Among DMARDs, leflunomide was most often prescribed $(1971,36.7 \%)$, followed by methotrexate $(1624,30.2 \%)$, sulfasalazine $(1221,22.7 \%)$, hydroxychloroquine $(1053,19.6 \%)$, azathioprine (297, 5.5\%), methylprednisolone $(248,4.6 \%)$ and ciclosporin $(125,2.3 \%) .77 \%$ of all prescriptions were with monotherapy . Monotherapy was most often recommended for AS (99.5\%), followed by RA and PsA (76.9\%) and LES (71.1\%), while combined therapy was recommended for LES (38.9\%) and RA and PsA (23.1\%). 19.7\% of all prescriptions had two DMARDs associated. More than half $(51.8 \%)$ of the DMARDs prescriptions were for elderly retired patients while almost a quarter $(20.4 \%)$ for patients with a handicap.

Conclusions: We have established that, in our cohort, the most prescribed drug in the treatment of autoimmune rheumatic diseases was leflunomide, followed closely by methotrexate. Monotherapy was preferred in the majority of cases, and mostly in the treatment of AS, while combined treatment was prescribed more often for patients with LES.

Further sources of information/References:

1. Winthrop KL, Strand V, Van der Heijde D, Mease P, Crow MK, Weinblatt $\mathrm{M}$ et al. The unmet need in rheumatology: reports from the Targeted Therapies meeting 2016. Clin Exp Rheumatol 2016;34(Suppl.98):S69-S76

2. Pincus T, Gibson KA, Castrejón I. Update on methotrexate as the Anchor Drug for Rheumatoid Arthritis. Bull Hosp Jt Dis 2013;71(Suppl 1): S9-19

3. Tunncliffe DJ, Singh-Grewal D, Kim S, Craig JC, Tong A. Diagnosis, Monitoring, and Treatment of Systemic Lupus Erythematosus: A systemic review of clinical practice guidelines. Arthritis Care Res.2015;67(10):1440-52

\section{Overview of Systematic Reviews of Therapeutic Ranges: Establishing the Threshold of Increased Drug Toxicity}

\section{J. Moss ${ }^{1}$, L. Cooney ${ }^{2}$, S. Golder ${ }^{3}$, Y. Loke ${ }^{4}$, A. Jorgensen ${ }^{5}$, I. Sinha ${ }^{6}$, D. Hawcutt ${ }^{6,7}$}

${ }^{1}$ Flintshire Children's Centre, Deeside, UK, ${ }^{2}$ University of Liverpool Medical School, Liverpool, UK, ${ }^{3}$ Department of Health Sciences, University of York, York, UK, ${ }^{4}$ School of Medicine, Health Policy and Practice, University of East Anglia, Norwich, UK, ${ }^{5}$ Department of Biostatistics, University of Liverpool, Liverpool, UK, ${ }^{6}$ Alder Hey Children's Hospital, Liverpool, UK, ${ }^{7}$ Department of Women's and Children's Health, University of Liverpool, Liverpool, UK

Background: Many medicines are dosed to achieve a particular therapeutic range. The lower and upper ends of the therapeutic range denote where efficacy is achieved, and where risk of drug toxicity increases beyond an acceptable frequency respectively. The evidence underpinning many therapeutic ranges is limited. Systematic reviews are the most robust method for evaluating and synthesizing existing literature, but there is currently no consensus on the optimal methodology for systematic reviews of therapeutic ranges.

Aim: To undertake an overview of systematic reviews of therapeutic ranges, and derive recommendations to optimise future systematic reviews.
Methods: The following databases were used: Cochrane Database of Systematic Reviews (CDSR), Database of Abstracts and Reviews of Effects (DARE) and MEDLINE. The published methodologies used when systematically reviewing the therapeutic range of a drug were analyzed. Step by step recommendations to optimize such systematic reviews were derived from this analysis.

Results: Ten systematic reviews that investigated the correlation between serum concentrations and clinical outcomes encompassing a variety of medicines and indications were identified. There were significant variations in the methodologies used. Only five reviews (50\%) assessed the correlation between serum levels and adverse events. These reviews were for: Aminophylline, Amisulpride, Mycophenolate Mofetil, Olanzapine and Thiopurine. Only one systematic review (10\%) established that the upper limit of the therapeutic range was able to predict drug related toxicity. Recommendations for future systematic reviews based on these findings were developed, and a model for interpreting systematic review data is proposed with regard to drug toxicity and the upper end of the therapeutic range.

Conclusions: The systematic reviews focused on clinical efficacy, with only $50 \%$ assessing adverse events. At present there are highly variable methodologies used, and there is no consensus of best practice when undertaking systematic reviews of therapeutic ranges. Using a standardised methodology will improve future research of therapeutic ranges.

\section{The Aspidium Project: A versatile App concept to support risk minimisation at patient level}

\author{
J-C. Delumeau ${ }^{1,2}$, G. Caron ${ }^{3}$ \\ ${ }^{1}$ ISoP, Singapore, Singapore, ${ }^{2}$ Bayer South East Asia, Singapore, \\ Singapore, ${ }^{3} A D N$, Singapore, Singapore
}

Introduction: Sub-optimal use of medicinal products may occur during ambulatory treatments suggesting routine risk minimisation methods, as currently designed and applied, may be insufficient to ensure adequate understanding of the treatment by the patient. On the other hand, adding risk minimisation interventions translates into burdens and costs that may limit their feasibility especially for products no longer promoted by a specific license holders. Used as vectors for enforcing risk minimisation, smartphones, provide an opportunity to overcome this issue.

Aim: The Aspidium Project, a satellite initiative of the ISoP SiG on Risk Minimisation for Asian

Countries, is aimed at developing an affordable IT platform including a versatile App intended for health authorities (HA) to support delivering risk minimisation contents, evaluating patients' understanding, also able to replay contents whenever needed.

Methods: The Aspidium platforms is intended to be appointed by specific HAs and customised.

It is designed for hosting HA-approved contents for multiple products whatever the licence holder and thus, capable of supporting risk minimisation for generic products. The App is primarily intended to support the treatment of the registered user or another family member e.g. child or elderly, the App user is taking care of. Once installed into the smartphone, the App interacts with a server located on a sizeable cloud.

Data trafficking is encrypted. However, locating the core of the system at the HA place is possible alternative to clouding. The Aspidium App may deliver diverse types of contents such as: (a) Textual: the approved Patient Information Leaflet maintained updated, (b) Video: e.g. what a Doctor would tell you on how to use the medicines under scope, (c) Key messages to be remembered, (d) Questionnaire to test the understanding of the proper use guidance, (e) Questionnaires aimed to collect real world 
evidence data reflecting effectiveness and/or tolerability, (f) Collect incident safety information occurring under treatment.

Activating the Treatment Assistant requires entering basic patient profile information upon patient's acceptance. Those data are to be stored into a password-protected area of the smartphone. Collecting information for public health purpose would require acceptance by the patient of a higher level of informed consent.

Results: A beta version of the Aspidium App will be presented at the 17th Annual ISoP Meeting.

Conclusions: Creating a self-funded consortium is intended to deploy and support clones of

Aspidium platforms in countries interested.

\section{Breastfeeding Women Taking Medications: About the Need to Collect Data}

\section{Gaboriau', M. Auffret ${ }^{1}$, J. Dekemp ${ }^{1}$, J. Béné ${ }^{1}$, S. Gautier ${ }^{1}$ \\ ${ }^{1}$ Regional Pharmacovigilance Center, Lille, France}

Background: Currently, breastfeeding is on the rise. World health organization (WHO), advise breastfeeding until 6 months of age. In France, promotion measures of breastfeeding led to a marked increase in prevalence and duration of breastfeeding. This increases the likelihood of taking a medication during this period. Whether breastfeeding should be stopped or not, the decision should be made on the basis of the available knowledge about risks associated with the drug for the infant. We are regularly questioned about the risk for infants exposed to drugs through breastfeeding. Benefit/risk balance is then left to the discretion of the prescriber. Aim: To describe cases of possible drug exposition during breastfeeding. Methods: We performed a retrospective descriptive analysis of inquiries about drug exposure during breastfeeding received by our regional pharmacovigilance centre of North of France, which cover 4 million of inhabitants, from January 1st 2016 to mars 31th 2017.

Results: Thirty-two calls about the risk associated with drug exposure in a lactating woman have been received during this period. Most of them were asked prospectively (27) and by healthcare professionals (26) but patients also questioned us. Breastfed infants were aged from a few days to one year old. It concerned mainly drugs (36) but also dietary supplements or phytotherapeutic products (3) or cosmetics (1). To note, 3 calls concerned possible adverse effects observed in infants through breastfeeding. Only 2 Summaries of product characteristics (SmPCs) allowed drug administration during lactation (amoxicilline, iomeprol) without restrictions while literature was somewhat reassuring in most cases. Recommendations from academic societies were found for 6 cases. In prospective issues, breastfeeding was not recommended for 3 drugs because of prematurity or a possible identified risk. Followup could be obtained for 7 cases and no adverse effect was observed in breastfed neonates.

Discussion/Conclusion: These data confirm the need for reliable information regarding drug exposure during lactation whereas SmPCs are not always accurate. For some drugs, authorized in only a few countries or used in orphan diseases, it is difficult to find information regarding drug exposure during breastfeeding. This highlights the need to collect and publish more data about drug administration in lactating women, even if there is an absence of adverse effect. That's why we, now, systematically monitor patients after request for information on the use of drugs during breastfeeding: to know if breastfeeding was pursued and, if applicable, if adverse effect occurred in breastfeed infants.
119 Proactive Pharmacovigilance: description of nivolumab's safety informations identified through the clinical data warehouse of a French University Hospital

\author{
H. Jantzem ${ }^{1}$, C. Le Guillou ${ }^{2}$, L. El Aridi ${ }^{3}$, A. Happe ${ }^{4}$, \\ D. Kowalski ${ }^{5}$, J.M. Cauvin ${ }^{6}$
}

${ }^{1}$ Regional Pharmacovigilance Centre, Brest, France, ${ }^{2}$ Medical Information Department, Brest, France, ${ }^{3}$ Regional Pharmacovigilance Centre, Brest, France, ${ }^{4}$ Medical Information Department, Brest, France, ${ }^{5}$ Regional Pharmacovigilance Centre, Brest, France, ${ }^{6}$ Medical Information Department, Brest, France

Background: Nivolumab belongs to a novel class of immunotherapy, PD1 inhibitors drugs. It was approved for advanced and metastatic melanoma and for advanced and metastatic non-small cell lung cancer. Aware of the need for further safety information about this new drug, we used the clinical data warehouse recently created in Brest University Hospital to identify all patients who received or are receiving nivolumab.

Objective/Aim: The aim of this work was to gather all the side effects by following up with each patient who received nivolumab as well as by monitoring patients currently receiving it.

Methods: Starting in January 1st, 2015 the hospital data warehouse was queried using the character string "nivolumab" (or equivalent names to account for potential typos). Each short extract of record that includes «nivolumab» would be displayed on a webpage with a link to the complete medical record. Note that this query is automatically refreshed on a weekly basis to account for new entries/records. This ensures an active follow up of new patients and those undergoing treatment. We collected by this way useful data like sex, age, indication, dates of treatment(s), number of cycles. Data related to side effects were closely monitored. Lastly, each medical record reporting adverse effects was subject to a notification which was recorded in the French Pharmacovigilance database.

Results: Between January 1st, 2015 and October 31st, 2016, 129 patients received at least one cycle of nivolumab, 84 men and 45 women. Indication was melanoma in $18 \%$ and lung cancer in $79 \%$ whereas 4 patients were treated for another indication. About $25 \%$ of patients experienced adverse effects. Many of them were serious and sometimes led to stop the treatment. The most common toxicities were thyroidal dysfunction, cutaneous and gastrointestinal side effects. Less common were hepatobiliary, musculoskeletal and hematological effects. Finally, the safety profile of nivolumab in this cohort of patients will be analyzed and discussed.

Conclusion: This experience in Brest University Hospital demonstrates the usefulness and relevance of using clinical data warehouse to follow up the introduction of new drugs in real life. It will certainly be an effective tool for active pharmacovigilance by helping to improve the knowledge of the drugs' risk profile which is needed to better manage newly approved drugs, especially in oncology.

\section{Patient ADR reporting through stakeholders' lens: experiences in different $\mathrm{EU}$ countries}

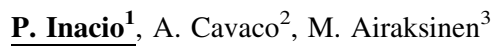 \\ ${ }^{1}$ Clinical Pharmacy Group, Division of Pharmacology \\ and Pharmacotherapy, Faculty of Pharmacy, University of Helsinki, \\ Helsinki, Finland, ${ }^{2}$ iMed.ULisboa Research Institute for Medicines
}


and Pharmaceutical Sciences, Faculty of Pharmacy, University of Lisbon, Lisbon, Portugal, ${ }^{3}$ Clinical Pharmacy Group, Division of Pharmacology and Pharmacotherapy, Faculty of Pharmacy, University of Helsinki, Helsinki, Finland

Introduction: Spontaneous reporting of adverse drug reactions (ADRs) is one method for the detection of emerging safety issue that may lead to a change in the benefit-risk profile of a medicine. Since 2012, in the European Union (EU) patients can directly report suspected ADRs to competent authorities. Five years after the legislation was enacted, patient reporting is increasing. However, this phenomenon has not been equal in all countries due to system maturation [1].

Aim: To explore reasons perceived by key stakeholders on levels of varying patient reporting of ADRs in four different EU countries, including implications on a common medicines safety policy.

Methods: A qualitative study was conducted with 12 representatives from national pharmacovigilance centres and/or authorities dealing directly with the analysis of patient report and national pharmaceutical industry representative bodies from Finland, Netherlands, Portugal and United Kingdom. Supranational organizations were also included, namely the World Health Organization, the Uppsala Monitoring Centre and the European Federation of Pharmaceutical Industries and Associations. Data was collected via faceto-face semi structured interviews and analyzed through an inductive thematic approach. The opinions expressed by the participants are personal and do not represent official positions of the institutions.

Results: Four themes (attitudes and beliefs, system maturation factors, regulatory improvements, and cultural shifts) emerged from data, and were found to be conceptually interconnected. Participants from countries that introduced patient reporting recently expressed a more negative attitude, if compared with ones that have had it for longer. All participants stated system maturation issues, such as the time to process this type of reports or other methodology factors, as pivotal on how effective the information provided by patients is used for safety purposes. Participants also highlighted the need for additional resources, both human and financial, to honestly address patient reporting and address its advantages.

Conclusions: Patient reporting of ADRs is seen fundamentally as relevant to most participants. The findings identified perceived barriers and facilitators of patient reporting. Despite the overarching opinions demonstrated across different stakeholder groups, there were contrary beliefs between the same stakeholder groups in different countries. Regardless of the positive aspects of patient reporting highlighted by the participants, the involvement of patients, use of information, and dissemination of this process of collecting ADRs among healthcare professionals and patients is far from optimal. A better integration of the work by the EU regulatory authorities is recommended.

Further sources of information/References

1. Inacio, P., A. Cavaco, and M. Airaksinen, The value of patient reporting to the pharmacovigilance system: a systematic review. $\mathrm{Br} \mathbf{J}$ Clin Pharmacol 2017; 83: p. 227-246

\section{Opinion of Costa Rican Pharmacists Regarding Some Aspects of the Spontaneous Adverse Drug Reactions reporting. 2017}

\author{
V. Hall ${ }^{1}$, L.G. Herrera ${ }^{1}$, M. Rocha ${ }^{1}$
}

${ }^{1}$ Pharmaceutical Care and Clinical Pharmacy Department, Pharmacy Faculty. University of Costa Rica, San Pedro, Montes De Oca, Costa Rica
Backgrounds: The importance of Pharmacovigilance (PV) for safe medicines and their safe use has increasingly been recognised during the last few years [1]. As part of the curriculum in the Pharmacy career of the University of Costa Rica, several aspects related to Pharmacovigilance are studied.

Aim: Describe the results of a survey to pharmacist in Costa Rica during the first half of 2017, regarding to PV.

Methods: Study Type: Cross-sectional study. Method: Application of a data collection instrument designed by the course Pharmacoepidemiology Topics teachers and applied by the students. The data were managed in a group, confidential and anonymous way.

Results: Information was collected from 38 pharmacist, of whom $47.37 \%$ work at private community pharmacy. $89.47 \%$ of the pharmacist are in contact with drug adverse reactions (ADR), but only $61.76 \%$ fill the Individual Case Safety Report (ICRS) and send it to the Costa Rican National Pharmacovigilance Center. The main reasons behind the no report of the ADR by the pharmacist are lack of time (23.81\%) and that the ADR is well known or is not serious $(23.81 \%)$. The main recommendations to improve the National Pharmacovigilance System (NPVS) are the following: training to professionals (26.87\%), campaigns to make patients aware of the importance of reporting the ADR (20.90\%) and the use technology to report online $(17.91 \%)$. In relation to PV regulations in Costa Rica, $63.16 \%$ of the pharmacist know about the existence of the one that creates the NPVS and 52.63\% about the one that regulated the Good Pharmacovigilance Practices.

Conclusions: The results show that pharmacists in Costa Rica have weaknesses to apply the spontaneous ADR reporting, so it is necessary to improve the NPVS by more training in how to report and the use of technological devices and software.

Discussion: In terms of training, the NPVS could develop a series of modules acording to the World Health Organization-International Society of Pharmacovigilance Pharmocovigilance Curriculum which provides an inventory and overview of the scope of pharmacovigilance $[1,2]$. Some challenges and barriers to promote PV in low and middle income countries, included the training [3], so the authors think that best way to start with those improvements is to make alliances between all the actors of the NPVS to make possible all those actions in a short period of time with the needed impact to the public health of the country.

Further sources of information/References:

1. Beckmann J, Hagemann U, Bahri $P$ et al. Teaching pharmacovigilance: the WHO-ISoP core elements of a comprehensive modular curriculum. Drug Saf 2014;37:743-59

2. Bate A, Beckmann J, Dodoo A et al. Developing a Crowdsourcing Approach and Tool for Pharmacovigilance Education Material Delivery. Drug Saf 2017 40:191-199

3. Olsson S, Pal S, Stergachis A, Couper M. Pharmacovigilance activities in 55 low- and middle-income countries: a questionnaire-based analysis. Drug Saf 2010;33:689-703

\section{Contribution of Adverse Events Reporting of HIV Antiretrovirals to VigiBase: Comparison between Sub- Saharan Africa and the Rest of the World}

\author{
A. Kuemmerle $^{\mathbf{1 , 2}}$, M. Maeder ${ }^{1,2}$, D. Sartori ${ }^{3}$, A. Lazarova ${ }^{1,2}$, \\ M. Urich ${ }^{1,2}$, A. Dodoo ${ }^{4}$, S. Olsson ${ }^{5}$, C. Burri ${ }^{1,2}$ \\ ${ }^{1}$ Swiss Tropical And Public Health Institute, Basel, Switzerland, \\ ${ }^{2}$ University of Basel, Basel, Switzerland, ${ }^{3}$ WHO Collaborating Centre \\ for International Drug Monitoring, Uppsala Monitoring Centre, \\ Uppsala, Sweden, ${ }^{4}$ Collaborating Centre for Advocacy and Training
}


in Pharmacovigilance, University of Ghana School of Medicine and Dentistry, Accra, Ghana, ${ }^{5}$ Sten Olsson Pharmacovigilance Consulting, Uppsala, Sweden

Introduction: The complexity of antiretroviral therapies (ART) with the inherent risk of developing adverse drug reactions asks for adequate drug safety monitoring [1-3]. The public health approach of providing access to HIV, tuberculosis and malaria drugs to patients infected in low-resource and high prevalence countries triggered the particular need for pharmacovigilance in those regions where it had often been neglected in the past $[4,5]$. This is part of the context in which WHO established the WHO Collaborating Centre for Advocacy and Training in Pharmacovigilance in Ghana in 2009 (WHO-CC Accra) and the WHO Collaborating Centre for Strengthening Pharmacovigilance Practices in Morocco in 2011 [6].

Aim: To describe the spontaneous adverse event reporting activities in Sub-Saharan Africa (SSA) before and after the implementation of WHO$\mathrm{CC}$ Accra and to compare the activities to those in the rest of the world (RoW).

Methods: The WHO Collaborating Centre for International Drug Monitoring, Uppsala Monitoring Centre (WHO-UMC) provided an extract of VigiBase including all individual case safety reports (ICSRs) with HIV antiretrovirals reported by member countries since the inception of the WHO Programme in 1968 until the 6th of October 2016. Data analysis was descriptive. The results are presented as the number of ICSR (nICSR). The nICSR was normalised according to 1) the duration of membership in the WHO Programme (in years) and 2) the number of HIV-infected people or number of people on ART in each region (source: UNAIDS global reports 2010 and 2013; only most recent available UNAIDS surveillance data used). Countries with no surveillance data were excluded from the respective analysis.

Results: In this dataset, 150'646 ICSRs issued by 104 WHO Programme member countries are recorded. Of these, $18.8 \%$ were coming from 28 SSA member states.

\begin{tabular}{llll}
\hline Reporting indicators & SSA & SSA & RoW \\
& $1987-2009$ & $2010-2016$ & $1987-2016$ \\
\hline nICSR & $2 ' 344$ & $26 ' 016$ & $122 ' 286$ \\
nICSR per Thousand HIV-infected & 0.10 & 1.15 & 13.04 \\
nICSR per year and per Thousand HIV-infected & 0.01 & 0.17 & 0.51 \\
nICSR per Thousand people on ART & 0.34 & 3.79 & $*$ \\
nICSR per year and per Thousand people on ART & 0.04 & 0.54 & $*$ \\
\hline${ }^{*}$ Number of people on ART not available for high-income countries, calculations not performed.
\end{tabular}

Conclusion: Reporting from SSA has impressively increased since WHOCC Accra was established in 2009 and later on the Moroccan Centre in 2011. However, SSA reporting is still below the reporting of the RoW. Efforts to develop and maintain pharmacovigilance systems to monitor HIV, as well as other disease medicines, must be strengthened.

Further sources of information/References:

1. Bakare N, Edwards IR, Stergachis A, Pal S, Holmes CB, Lindquist M, et al. Global pharmacovigilance for antiretroviral drugs: overcoming contrasting priorities. PLoS Med 2011;8:e10010542. Miller V, Nwokike J, Stergachis A. Pharmacovigilance and global HIV/AIDS. Curr Opin HIV AIDS 2012;7:299-304

2. Rachlis B, Karwa R, Chema C, Pastakia S, Olsson S, Wools-Kaloustian $\mathrm{K}$, et al. Targeted Spontaneous Reporting: Assessing Opportunities to Conduct Routine Pharmacovigilance for Antiretroviral Treatment on an International Scale. Drug Saf 2016;39:959-764. Olsson S, Pal SN, Dodoo A. Pharmacovigilance in resource-limited countries. Expert Rev Clin Pharmacol 2015;8:449-605. Pirmohamed M, Atuah KN, Dodoo AN, Winstanley P. Pharmacovigilance in developing countries. BMJ. 2007;335:462 6. Ampadu HH, Hoekman J, de Bruin ML, Pal SN, Olsson
S, Sartori D, et al. Adverse Drug Reaction Reporting in Africa and a Comparison of Individual Case Safety Report Characteristics Between Africa and the Rest of the World: Analyses of Spontaneous Reports in VigiBase(A (R)). Drug Saf 2016;39:335-45

\section{Spontaneous Reporting characteristics of HIV Antiretrovirals in VigiBase: Disproportionate Analysis Between Sub-Saharan Africa and the Rest of the World}

A. Kuemmerle ${ }^{\mathbf{1 , 2}}$, D. Sartori ${ }^{3}$, M. Maeder ${ }^{1,2}$, A. Lazarova ${ }^{1,2}$, M. Urich ${ }^{1,2}$, A. Dodoo $^{4}$, S. Olsson ${ }^{5}$, C. Burri ${ }^{1,2}$

${ }^{1}$ Swiss Tropical And Public Health Institute, Basel, Switzerland, ${ }^{2}$ University of Basel, Switzerland, ${ }^{3}$ WHO Collaborating Centre for International Drug Monitoring, Uppsala Monitoring Centre, Uppsala, Sweden, ${ }^{4}$ WHO Collaborating Centre for Advocacy and Training in Pharmacovigilance, University of Ghana School of Medicine and Dentistry, Accra, Ghana, ${ }^{5}$ Sten Olsson Pharmacovigilance Consulting, Uppsala, Sweden

Introduction: According to the latest UNAIDS statistics, 9.7 Million people were receiving antiretroviral therapies (ART) in low- and middleincome countries in 2012. Of these, 7.5 Million were living in Sub-Saharan Africa (SSA) [1]. Toxicological profiles of drugs primarily developed to treat people in the north cannot be interpreted similarly in lowresource contexts where populations have different epidemiological or demographic profiles [2]. Well running pharmacovigilance systems in remote and low-resource settings are therefore essential to detect any safety issue and improve the safe use of ART in these populations [3, 4]. Aim: To compare the individual case safety report (ICSR) characteristics of HIV-antiretrovirals between Sub-Saharan Africa (SSA) and the rest of the world (RoW).

Methods: Data was extracted and analysed by the WHO Collaborating Centre for International Drug Monitoring, Uppsala Monitoring Centre (WHO-UMC) from VigiBase. The dataset included all ICSRs reported on HIV antiretrovirals since the inception of the WHO Programme in 1968 until the $19^{\text {th }}$ of April 2017. vigiPoint, a method developed by WHO-UMC using disproportionalities to compare the SSA subset to the RoW was applied to analyse the data [5]. Results are presented as log odds ratios $(\log \mathrm{OR})$ with $95 \%$ confidence intervals $(95 \% \mathrm{CI})$. The level of significance was set for $\log$ OR $>0.5$.

Results: Reporting was spontaneous in $95 \%$ of the cases in SSA compared to $86 \%$ in RoW $(\log$ OR $=1.59$ [1.56-1.61]). Adverse events classified in the MedDRA System Organ Class related to skin and subcutaneous disorders and nervous disorders were more often reported in SSA. Older drugs from the 1990's such as stavudine, nevirapine and tri-therapies containing AZT-3TC and nevirapine were more often reported in SSA.

\begin{tabular}{lllll}
\hline Summary of some ICSR characteristics of interest (not exhaustive) & SSA & RoW & Log OR $[95 \% \mathrm{Cl}]$ \\
\hline Notifier & Physician & $31 \%$ & $62 \%$ & $-1.81[-1.85 ;-1.78]$ \\
& Pharmacist & $32 \%$ & $10 \%$ & $1.97[1.93 ; 2.00]$ \\
& Other health professional & $36 \%$ & $12 \%$ & $1.95[1.91 ; 1.98]$ \\
\hline \multirow{2}{*}{ Gender } & Female & $68 \%$ & $36 \%$ & $1.88[1.85 ; 1.91]$ \\
& Male & $32 \%$ & $64 \%$ & $-1.91[-1.94 ;-1.87]$ \\
\hline Age group & Children (2 to 11 years) & $3 \%$ & $1 \%$ & $0.79[0.68-0.90]$ \\
\hline
\end{tabular}

Conclusion: Reporting from non-physician health professionals should be further investigated for future capacity building strategies in SSA. As expected, reported products and SOC are varying between SSA and RoW. Finally, a specific analysis should be addressed to children and women of 
child bearing potential as these vulnerable sub-populations might present a different toxicological profile.

Further sources of information/References:

1. UNAIDS. Global Report 2013

2. Miller V, Nwokike J, Stergachis A. Pharmacovigilance and global HIV/AIDS. Curr Opin HIV AIDS 2012;7:299-3043. Ampadu HH, Hoekman J, de Bruin ML, Pal SN, Olsson S, Sartori D, et al. Adverse Drug Reaction Reporting in Africa and a Comparison of Individual Case Safety Report Characteristics Between Africa and the Rest of the World: Analyses of Spontaneous Reports in VigiBase(A (R)). Drug Saf 2016;39:335-45

3. Isah AO, Pal SN, Olsson S, Dodoo A, Bencheikh RS. Specific features of medicines safety and pharmacovigilance in Africa. Ther Adv Drug Saf 2012;3:25-34

4. Juhlin K, Star K, Norén GN. Pinpointing Key Features of Case Series in Pharmacovigilance. Drug Saf 2013;36:912-3

\section{Risk Management by Using ADRs Reports via Social Media}

\author{
$\underline{\text { Y. Oppamayun }}{ }^{1}$, P. Pokakul, S. Nootim, C. Sonsuphap \\ ${ }^{1}$ Health Product Vigilance Center, Thaifda, Nonthaburi, Thailand
}

Introduction: Social network, Line Application, is widely used by pharmacist working groups in provinces for sharing ADRs data and use as the new channel to report ADRs from local to National Pharmacovigilance Center (HPVC) in Thailand. Because of convenient and rapid way to send data to national center, taking actions for minimize all risks are expected to be done fast.

Aims: Explore the number and quality of ADRs reports sent via Line Group, and analyze measures of risk management from Thai FDA.

Methods: We use data received by Line Group from 01/01/2014 to 01/04/ 2017, categorize ADRs, causality assessment and summarize each case for making risk management .Quality of reports is graded by using minimum criteria which are product name, ADRs, patient, and reporter. Measures of risk management are classified into 3 groups; legal measure, communication measure and regular measure.

Results: 17 ADRs reports were received to HPVC via Line Group. There were 12 reports of health supplement products, including herb and 5 reports of medicines. Problems found in health supplement products were medicines which illegal as food ingredients, sibutramine, which was withdrawn several years ago, and steroid. We analyzed reports; suspected product and ADRs, all were known ADRs except health supplements which contain several herbs may cause unknown adverse reactions. Quality of health supplement reports met minimum criteria, but lack of important information such as clinical data, underlying disease, so conclusion would not be done quickly. Five reports were medicines, 4 of these named "yachut" which were popular used in rural. 2 of them showed indication for muscles and joints pain relieve, information of patient diseases such as diabetes, CKD and suspected medicine, dexamethasone, which caused known ADRs, gastrointestinal bleeding. Reports were good quality, but limited for make decision whether medicines or illness caused patient death. 2 reports showed indication for weight loss, and found sibutramine. The last one was eye drops contaminated with bacteria caused patient blindness. The product was substandard, quality of report was very good.

We classified risk management into 3 measures; legal, communication and regular monitoring. 14 cases were legal measures; illegal medicine, sibutramine, and substandard medicine. 2 cases were in communication measures; awareness of warning labels. One case was in regular monitoring for further information.

Conclusion: Social media could be one of PV tools for getting new information to make risk management properly. By facilitate staff using it and set standard for report pattern sharing among team.

127 Use of a medicine chart in promoting the safe use of Antiretrovirals amongst adults at Mbagathi Hospital in Nairobi, Kenya

\author{
C. Wambura \\ ${ }^{1}$ Mbagathi Hospital, Nairobi, Kenya
}

Background: Antiretrovirals are the mainstay treatment for patients living with HIV globally. In public hospitals in Kenya, the commodities are supplied through the Kenya Medical Supply Agency. Due to the procurement guidelines, different brands of Antiretrovirals are usually made available for use which sometimes translates to the same drug being supplied in more than one brand with a different package and sometimes a different color. This results in a situation where the clinicians and patients are not easily able to identify some of the antiretrovirals available at that particular point in time and thus requiring assistance from the pharmacist to help in identification of the medication.

Aim: To make available a medicine chart with the pictures of the antiretroviral packs alongside the accompanying tablets indicating the different brands that have been available for use in the pharmacy which will help in easy identification of the drugs by both the clinicians and the patients and thus omit medication errors and adverse events.

Methods: A survey was carried out to identify the various brands of the antiretrovirals that were available in the comprehensive care centre Pharmacy and the results were documented in tabular form which was the basis of the chart.

Results: The total number of antiretroviral drugs available for use by adults were 10 out of which, 8 had been supplied in more than one brand. On average, two brands were available per drug.

Conclusion: Most antiretrovirals available for use by adults at Mbagathi Hospital have more than one brand and thus the use of a medicine chart can help in easy identification of the drugs by the clinicians and patients and ultimately promote the safe use of these drugs.

\section{Off-label drug use and Adverse Drug Events (ADEs) in paediatric patients: An analysis of the Jordanian National Pharmacovigilance Database}

\author{
S. Al-zubiedi ${ }^{1}$, N. Bawaresh ${ }^{2}$ \\ ${ }^{1}$ The University of Jordan, Amman, Jordan, ${ }^{2}$ Jordan Food and Drug \\ Administration, Amman, Jordan
}

Introduction: Off-label drug use in pediatrics is common due to lack of pediatrics clinical trial and safety testing. Although, it provides the best available treatment for pediatrics where drugs have not been evaluated for their safety, drug administration outside the conditions assessed during clinical trials may result in significant consequences i.e. adverse drug events (ADEs) [1].

Aims: to assess the impact of off-label drug use on ADEs in paediatric patients using the Jordanian National Pharmacovigilance Database. 
Method: All spontaneous reports of ADEs in paediatrics patients (i.e. age $<18$ years) recorded in the National Jordanian Pharmacovigilance Programme Database between January 2013 and December 2015 were investigated. Reports with missing age or event details were excluded. All reports included were assessed in order to define each primary and secondary suspected drug as either authorized, off-label, or unknown. Offlabel was defined as the use of a drug outside the terms of the drug labeling/licensing [2]. In the current analysis, an adverse event was considered "serious" if it resulted in inpatient hospitalization or prolongation of existing hospitalization.

Results: Between January 2013 and December 2015, 791 valid ADEs in paediatrics were reported to the Jordanian Pharmacovigilance Program. Overall, off-label drug use was responsible for $74 \%$ of the reported ADEs in paediatrics. Of all off-label ADEs, anticancer (84\%), immunosuppressant $(63 \%)$, and antimicrobial (36\%) were the most commonly implicated classes of medications. Comparing the proportions of off-label prescribing among different age groups and drug classes, ADEs were significantly more frequent in infants $(\mathrm{P}=0.002)$, and among anticancer drugs $(\mathrm{p}<0.001)$.

Off-label drug use was implicated in $200(83 \%)$ of all serious reactions i.e. resulted in hospital admissions. ADEs associated with off-label prescription were more likely to be implicated in hospital admissions than authorized drugs (RR 1.73, 95\% CI 1.29, 2.34, P <0.001). After excluding anticancer drugs, risk of off-label medicine being implicated in an ADE remained significantly greater than that for a licensed drug (relative risk $1.13,95 \%$ CI $1.04,1.22, \mathrm{P}=0.02$ )

Conclusion: off-label drug use is more likely to be implicated in an ADEs than authorized medicines in pediatrics. Overall, off-label drug use in pediatrics is associated with increased risk of hospitalization. Our study highlights the needs to improve the study of drug safety in the pediatric population.

Further sources of information/References:

1. Turner S, Nunn AJ, Fielding K, Choonara I. Adverse drug reactions to unlicensed and off-label drugs on paediatric wards: a prospective study. Acta Paediatr 1999;88:965-8

2. Bellis JR, Kirkham JJ, Thiesen S, Conroy EJ, Bracken LE, Mannix HL, Bird KA, Duncan JC, Peak M, Turner MA, Smyth RL, Nunn AJ, Pirmohamed M. Adverse drug reactions and off-label and unlicensed medicines in children: a nested case-control study of inpatients in a pediatric hospital. BMC Med 2013; 11: 238

\section{Post-marketing Safety of Deferasirox in Combo vs Monotherapy, a Comparative Assessment}

\author{
L. Velez-Nandayapa ${ }^{1,2}$, G. Holder ${ }^{2}$, R. Singal ${ }^{3}$, K. Menon ${ }^{3}$, \\ A. Cortoos ${ }^{4}$, M. Shi ${ }^{4}$, J. Eisinger ${ }^{2}$ \\ ${ }^{1}$ University of Basel, Zurich, Switzerland, ${ }^{2}$ Novartis Pharma AG, \\ Basel, Switzerland, ${ }^{3}$ Novartis, Hyderabad, India, ${ }^{4}$ Novartis \\ Pharmaceuticals Corporation, New Jersey, USA
}

Introduction: Deferasirox (DFX) is a once-daily oral iron-chelator approved as monotherapy (MONOTX) for transfusional iron overload and non-transfusion dependent thalassemia syndromes. Combination therapy (COMBOTX) with two iron-chelators is an option for patients when MONOTX proves inadequate or in patients with severe iron overload who are at risk of organ function failure due to iron related toxicity. The positive benefit-risk profile of DFX in COMBOTX with deferoxamine has been shown in patients with severe myocardial and/or liver iron overload
(Ayidinok 2015, Cassinerio 2014), but further safety assessment of COMBOTX vs MONOTX is warranted.

Aim: Our study aimed to provide reliable assessment of the safety profile of DFX in COMBOTX vs MONOTX in post-marketing setting.

Methods: A cumulative (Nov2005-Jun2016) assessment of the Novartis safety database (NSD) was performed using reporting rate (cases [by $\mathrm{PT}] /$ patient exposure). The reporting rate ratio (RRR) was used as a measure of relative effect with $95 \%$ confidence intervals $(95 \% \mathrm{CI})$ and $p$ values $\left(\right.$ from $\mathrm{x}^{2}$ ). The search strategy included 13 Standard MedDRA Queries and 3 High Level Terms in 7 groups according to identified/ potential risks for DFX. The NSD outputs were divided in COMBOTX vs MONOTX cases.

Results: Searches retrieved for COMBOTX/ MONOTX (cases/events) $146 / 622$ vs $36,416 / 104,349$ with patient exposure of $1,605 / 319,371$ patient-years during 10.7 years of evaluation. No associations were found against COMBOTX in the 7 risks evaluated. Overall results are shown in Table 1.

Conclusions: The use of DFX off-label COMBOTX vs MONOTX is low with a relation 1:250 cases. Although the safety profile of DFX in COMBOTX vs MONOTX seems similar, these results should be interpreted with caution due to the small number of COMBOTX cases.

Table 1. OR analysis by risk in combo vs monotherapy.
\begin{tabular}{|l|l|l|l|}
\hline Risk & $\begin{array}{l}\text { Events } \\
\text { COMBOTX/ } \\
\text { MONOTX }\end{array}$ & $\begin{array}{l}\text { OR } \\
\mathbf{( 9 5 \% C I )}\end{array}$ & p-value \\
\hline $\begin{array}{l}\text { Renal disorders, (inc. serum } \\
\text { creatinine, acute renal failure, renal } \\
\text { tubular disorders (acquired Fanconi's } \\
\text { synd) }\end{array}$ & $37 / 6,440$ & $1.22(0.89-1.68)$ & 0.22 \\
\hline Inc. liver transaminases. & $13 / 2,463$ & $1.05(0.61-1.81)$ & 0.86 \\
\hline Hepatic failure & $5 / 1,009$ & $0.99(0.41-2.37)$ & 0.98 \\
\hline GI hemorrhage and ulcers; esophagitis & $10 / 1,299$ & $1.53(0.82-2.85)$ & 0.18 \\
\hline Hearing loss & $1 / 433$ & $0.46(0.06-3.27)$ & 0.44 \\
\hline $\begin{array}{l}\text { Lens opacities, retinal changes \& optic } \\
\text { neuritis }\end{array}$ & $5 / 969$ & $1.03(0.43-2.47)$ & 0.96 \\
\hline Blood cytopenias & $24 / 6,547$ & $0.73(0.49-1.09)$ & 0.12. \\
\hline
\end{tabular}

130 Infections and serious infections as risks of rituximab in rheumatoid arthritis. Results from an updated meta-analysis of randomised clinical trials

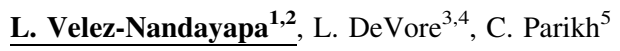

${ }^{1}$ University of Basel, Zurich, Switzerland, ${ }^{2}$ Novartis Pharma AG,

Basel, Switzerland, ${ }^{3}$ Drug Safety Research Unit, Southampton, UK,

${ }^{4}$ University of Portsmouth, Portsmouth, UK, ${ }^{5}$ Novartis, Hyderabad, India

Introduction: The association between serious infections (SINFs) and the use of rituximab (RTX) in patients with lymphoma and other haematological malignancies has been clearly confirmed; but, there is uncertainty about the association of SINFs with RTX in rheumatoid arthritis (RA). As in other previous meta-analysis, there have not been evidence of association of SINFs and RTX vs placebo (PCB) in RA; however, this lack of evidence (Hernández-Cruz, 2011) has been hypothesized, could be a type II statistical error.

Aim: Our study, a systematic review and meta-analysis (SR\&MA), aimed to provide reliable assessment of the risk of total infections (TINFs), infections (INFs) \& SINFs and RTX in RA (RTX-RA). 
Methods: This SR\&MA was registered with PROSPERO database (CRD42014015655) as protocol for a complete evaluation of the safety profile of RTX-RA. The search strategy involved randomised clinical trials using rituximab in RA, and it was performed from January 1990 to January 2017 in Medline, EMBASE and Cochrane Library databases. This SR\&MA was conducted following the PRISMA statement (Preferred Reporting Items for Systematic reviews and Meta-Analyses). The outcomes evaluated were: the number of TINFs, INFs \& SINFs reported as outcomes of interest. Analysis of odds ratio (OR) as measure of effect with random effect models and 95\% confidence intervals (CI 95\%) and $p$ values as generated from the chi-squared were calculated; heterogeneity was assessed using the $\mathrm{I}^{2}$ test. Sub-analysis by RTX low and high doses (500 vs $1000 \mathrm{mg}$ ) were performed.

Results: Eight publications were included in this SR\&MA involving 3030 subjects. The event's number in RTX + methotrexate (RTX-MTX) group / control PCB+MTX for TINFs, INFs \& SINFs were 798/412; 752/382; and $46 / 30$ events. We found no evidence of association between RTX-MTX and TINFs, INFs \& SINFs. Nor did the sub-analysis by RTX doses 500 vs $1000 \mathrm{mg}$. Our results are shown in table 1.

Table 1. OR analysis for type of infection

\begin{tabular}{|l|l|l|l|}
\hline Infection type & OR & $\mathbf{9 5 \% C l}$ & p-value \\
\hline TINFs & 1.02 & $0.81-1.29$ & 0.861 \\
\hline INFs & 1.04 & $0.84-1.29$ & 0.740 \\
\hline SINFs & 0.71 & $0.44-1.14$ & 0.155 \\
\hline
\end{tabular}

Conclusions: Our results suggest no evidence of association between RTX-MTX in RA and TINFs, INFs \& SINFs and the possibility of type II error unlikely.

\section{ISoP Global Standard for Career Framework in Pharmacovigilance-Special Interest Group Status Report}

\section{J. Petracek ${ }^{1}$}

${ }^{1}$ European Pharminvent Services, Prague, Czech Republic, ${ }^{2}$ Institute of Pharmacovigilance, Prague, Czech Republic, ${ }^{3}$ PrimeVigilance, Guildford, UK

Introduction: Recruiters and managers face a growing challenge of selecting the right candidates for more and more demanding pharmacovigilance roles. Despite many educational activities, including formal university degree programmes, as well as membership programmes of a few national professional organisations, there is no global standard for pharmacovigilance career framework serving the global industry, academia, government and non-profit organisations.

Aim: To develop a globally recognised Standard for Career Framework in Pharmacovigilance assisting human resources and managers with selection of the right candidates for global pharmacovigilance roles, as well as with setting the optimal training programmes and succession plans for their employees.

Methods: ISoP Special Interest Group dedicated to the topic has been approved by the Executive Committee. The first part of the work takes place in 3Q of 2017, building on the existing successful ISoP PV curriculum, WHO educational activities, as well as number of educational programmes available for pharmacovigilance professionals. Standardisation will then work on critical elements to be included in the Global Standard for Career Framework in Pharmacovigilance.
Results: The work is planned for 2 years. At the ISoP Annual Meeting 2017, the first part of the work would be presented. This should include comprehensive overview of all internationally recognised pharmacovigilance educational programmes, and existing career structures in major organisations. Based on this background research, a detailed plan for development of the Global Standard for Career Framework in Pharmacovigilance would be presented.

Conclusions: The work starts in 3Q 2017 and is planned for 2 years. With the presentation of the Status Report the stimulated ISoP community interest may further contribute to the successful outcome of this SIG and help establish ISoP as globally recognised professional organisation managing Global Standard for Career Framework in Pharmacovigilance.

\section{Selective Serotonin Reuptake Inhibitors (SSRIs) and major congenital heart defects (CHDs): An analysis of FDA Spontaneous Adverse Event Report Databases}

\author{
S. Al-zubiedi ${ }^{1}$ \\ ${ }^{1}$ The University of Jordan, Amman, Jordan
}

Introduction: selective serotonin reuptake inhibitors (SSRIs) are commonly prescribed in pregnant women to treat various conditions. However, conflicting evidence exist regarding their safety and the potential links between their use during pregnancy and the risk of major congenital anomalies (CAs), particularly congenital heart defects (CHDs).

Aims: to assess the association between SSRI (Paroxetine, Citalopram, Escitalopram, Fluoxetine, Paroxetine, and sertraline) use during pregnancy and the risk of major CAs and CHDs by assessing spontaneous reports submitted to the publicly available FDA Adverse Events Reporting System (FAERS)

Methods: FAERS data reporting major CAs including CHDs in the period from the first quarter of 2004 through second quarter of 2014 were extracted. Major CAs were classified according to the EUROCAST guide 1.3 [1] and ICD10. Reports with missing event details, ambiguous drug name, and duplicate reports were excluded from the analysis. A case by non-case disproportionality approach was used whereby a Reporting Odds Ratio (ROR) with $95 \%$ confidence interval (CI) was calculated. When the lower bound of the $95 \%$ CI was $>1$, significant disproportionality was considered to be present [2].

Results: major CAs were recorded in 12,120 ( $0.4 \%$ of the entire FAERS) of which 4655 (38.4\%) reports mentioned at least one SSRI as suspect agent for major CAs. Selective serotonin reuptake inhibitors (SSRIs) use was reported in $45.8 \%$ of major CHDs cases. Paroxetine $(29.9 \%)$, sertraline $(13.4 \%)$, and fluoxetine $(8.5 \%)$ were the most commonly implicated SSRIs in major CHD cases. Overall, statistically significant ROR emerged for SSRI as a class with an increased risk of major CHD (ROR $=4.08,95 \%$ IC, 3.77, 4.41), escitalopram $(\mathrm{ROR}=2.09,95 \% \mathrm{CI}$ 1.57-2.78), fluoxetine ( $\mathrm{ROR}=1.5895 \% \mathrm{CI}, 1.38,1.83$ ), paroxetine $(\mathrm{ROR}=3.83,95 \%$ CI 3.47, 4.23), and sertraline $(\mathrm{ROR}=2.74,95 \% \mathrm{CI}$, 2.4,3.13). The ROR of major CHD associated with the use citalopram was not statistically significant $(\mathrm{ROR}=0.99,95 \%$ CI $0.75-1.33$ ).

Conclusions: the current analysis using FAERs shows that SSRIs, except citalopram, use during pregnancy is associated with increased risk of major CHDs. The use of SSRIs was not associated with increased risks of other major CAs. However, more epidemiological studies are required to confirm this association.

Further sources of information/References

1. EUROCAT. EUROCAT European surveillance of congenital anomalies. Guide 1.3. EUROCAT European surveillance of congenital 
anomalies. Guide 1.3. EUROCAT Central Registry, University of Ulster, Newtownabbey, Co Antrim, UK. Available: http://www. eurocat-network.eu/content/EUROCAT-Guide-1.3.pdf. 2005. Accessed 1 May 2017

2. Almenoff JS, Pattishall EN, Gibbs TG, DuMouchel W, Evans SJ, Yuen N. Novel statistical tools for monitoring the safety of marketed drugs. Clin Pharmacol Ther 2007; 82: 157-66

\section{A clinician network for sharing patients experiences in pharmacovigilance: the PHARMACOWIKILANCE project}

I. Convertino ${ }^{1}$, M. Galiulo ${ }^{1}$, A. Capogrosso Sansone ${ }^{1}$, A. Marino $^{1}$, T. Knezevic ${ }^{2}$, S. Pieroni ${ }^{2}$, Professor C. Blandizzi ${ }^{1,3}$, M. Tuccori ${ }^{3}$

${ }^{1}$ Division of Pharmacology and Pharmacovigilance, Department of Clinical and Experimental Medicine, University of Pisa, Pisa, Italy, ${ }^{2}$ Institute of Clinical Physiology, National Research Council, Pisa, Italy, ${ }^{3}$ Unit of Adverse Drug Reactions Monitoring, University Hospital of Pisa, Pisa, Italy

Introduction: "Medical wiki", including platforms or similar online resources, allows clinicians to add and edit medical information collectively [1]. After thalidomide tragedy, "experience sharing" become one the fundamental elements of Pharmacovigilance philosophy. In line with this, the application of a "wiki" model to Pharmacovigilance activities seems an appealing strategy to implement drug safety awareness among healthcare professionals.

Aim: To develop and run a networking platform (Pharmacowikilance) for the monitoring of adverse drug reactions.

Methods: The Pharmacowikilance platform has been developed to be a patient management tool that can be used in any Hospital ward or primary care. The platform was organized in the following sections: patient demographics, patient medical history, hospital or emergency department or primary care admissions depending on study settings, therapies, adverse drug events, drug-drug interactions and laboratory investigations. The platform use hierarchical-based strategy for online accessing to the information that preserve patient privacy and provide the possibility of sharing clinical experience among healthcare professionals participating to the network. Active monitoring of a drug, a group of drugs or a specific type of patients can be proposed by the coordinating centre or even the peripheral centres. Then a network of centres interested in this monitoring is created and the follow up starts. Patients data can be entered from already existing medical records (manually or automatically) or prospectively after the signature of an informed consent. The coordinating centre supports the identification and the reporting to the national health authority of any adverse drug reaction (ADR).

Results: The Pharmacowikilance platform was released in 2015 and its use is ongoing. A total of 4 safety observational studies are carried out, 6 centres were included (1 Pisa, 1 Pistoia, 3 Firenze, 1 Siena) and 4.582 patients are recorded in the database on April 30st, 2017. These studies were performed in the following settings: oncology wards, geriatric wards, emergency departments and primary care. Over the study period, 468 suspected ADRs have been captured by the network.

Discussion: Pharmacowikilance platform is a quick and easy tool for clinicians to manage patient care and to collect data for drug safety in observational studies.
Conclusion: Pharmacowikilance platform allows quality data check and update in real time, it has improved the completeness of patients records. This platform has created a network of healthcare professionals that can easily share their experience upon request.

Further sources of information/References

1. Giustini D. Web 3.0 and medicine. Bmj. 2007;335:1273-4

\section{Biological substantiation of antipsychotic- associated pneumonia: literature review and computational analyses}

\section{J. Sultana $^{\mathbf{1 , 2}}$, M. Calabro, ${ }^{1}$, R. Garcia-Serna ${ }^{3}$, C. Ferrajolo ${ }^{2,4}$, C. Crisafulli, J. Mestres ${ }^{3,5}$, G. Trifiro, ${ }^{1,2}$}

${ }^{I}$ Department of Biomedical and Dental Sciences and Morphofunctional Imaging, University of Messina, Messina, Italy, ${ }^{2}$ Department of Medical Informatics, Erasmus Medical Centre, the Netherlands, ${ }^{3}$ Chemotargets SL, Spain, ${ }^{4}$ Department of Experimental Medicine, Unit of Clinical Pharmacology, Campania Regional Center of Pharmacovigilance and Pharmacoepidemiology, University of Campania, Naples, Italy, ${ }^{5}$ Research Group on Systems Pharmacology, Research Program on Biomedical Informatics, IMIM Hospital del Mar Medical Research Institute and University Pompeu Fabra, Parc de Recerca Biomèdica de Barcelona, Barcelona, Spain

Background: Antipsychotic (AP) safety has been widely investigated. However, mechanisms underlying AP-associated pneumonia are not welldefined.

Objectives: The aim of this study was to investigate the known mechanisms of AP-associated pneumonia through a systematic literature review, confirm these mechanisms using an independent data source on drug targets and attempt to identify novel AP drug targets potentially linked to pneumonia.

Methods: A search was conducted in Medline and Web of Science to identify studies exploring the association between pneumonia and antipsychotic use, from which information on hypothesized mechanism of action. Public repositories of pharmacology and drug safety data were used to identify the receptor binding profile and safety events of the following APs commonly investigated in studies on the risk of AP-associated pneumonia: amisulpride, clozapine, haloperidol, olanzapine, quetiapine, risperidone, and zotepine. Cytoscape was then used to map biological pathways that could link AP targets and off-targets to pneumonia.

Results: The literature search yielded 200 articles; 41 were considered relevant. Thirty studies reported a hypothesized mechanism of action, most commonly activation/inhibition of cholinergic, histaminergic and dopaminergic receptors. In vitro pharmacology data confirmed receptor affinities identified in the literature review. Two targets, thromboxane A2 receptor (TBXA2R) and platelet activating factor receptor (PTAFR) were found to be novel AP target receptors potentially associated with pneumonia. Biological pathways constructed using Cytoscape identified plausible biological links potentially leading to pneumonia downstream of TBXA2R and PTAFR.

Conclusions: Innovative approaches for biological substantiation of drugadverse event associations may strengthen evidence on drug safety profiles and help tailoring pharmacological therapies. 
135 Adverse drug reactions in oncoLogy: intEnsive monitoring program on biotechnological anD taRget therapies in Oncologic patientS-ALEXANDROS observational study

M. Galiulo ${ }^{1}$, I. Convertino ${ }^{1}$, C. Blandizzi ${ }^{1,8}$, R. Tassi ${ }^{2}$, S. Diacciati ${ }^{3}$, S. Nobili ${ }^{4}$, A. Capogrosso Sansone ${ }^{1}$, A. Alessandra

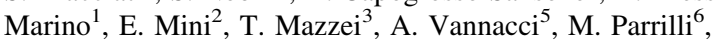
T. Corona ${ }^{7}$, M. Tuccori ${ }^{8}$

${ }^{1}$ Division of Pharmacology and Pharmacovigilance, Department of Clinical and Experimental Medicine, University of Pisa, -Pisa, Italy, ${ }^{2}$ Unit of Translational Oncology, Careggi University Hospital of Florence, Florence, Italy, ${ }^{3}$ Unit of Oncological Pharmacology, Careggi University Hospital of Florence, Florence, Italy,

${ }^{4}$ Department of Health Sciences, University of Florence, Florence, Italy, ${ }^{5}$ Department of Neurosciences, Psychology, Drug Research and Child Health, Section of Pharmacology and Toxicology, University of Florence, Florence, Italy, ${ }^{6}$ Tuscan Regional Centre of Pharmacovigilance, Pharmacy Department, Health District, Florence, Italy, ${ }^{7}$ Tuscan Region, Section for drug policies and appropriateness, Florence, Italy, ${ }^{8}$ Unit of Adverse Drug Reactions Monitoring, University Hospital of Pisa, Pisa, Italy

Introduction: Oncologic patients are particularly susceptible to adverse drug reactions (ADRs) [1]. Their sensitivity to ADRs results from: narrow therapeutic index of some chemotherapeutics, multidrug regimens and comorbidities. Malnutrition and organ dysfunctions could also affect drug pharmacokinetics making drug safety less predictable [2]. Therefore, monitoring oncologic patients' safety in clinical practice should be a priority [3].

Aim: To evaluate occurrence of ADRs and potential drug-drug interactions (DDIs) associated to antineoplastic target therapies.

Methods: A retrospective, observational, no-profit study (ALEXANDROS), was carried out at the Unit of Oncology of the University Hospital in Florence. Adult patients accessing from July 2014 to July 2016 with target therapies for solid tumour indications (Anatomical Therapeutic Classifications: L01XC-monoclonal antibodies; L01XE-protein kinase inhibitors) were included. Patients' data were retrieved from medical records using the Patient Data Form available on Pharmacowikilance (a platform developed by the Tuscan Regional Centre of Pharmacovigilance), including demographic information, medical history, pharmacological treatments, and ADRs. Potential DDIs were assessed by checking DDIs on Micromedex ${ }^{\circledR}$.

Results: Overall, 130 Caucasian cancer patients, mean age 64.28 years (standard deviation, SD \pm 12.12 ), with the following primary cancer diagnosis: 39 colorectal cancer, 27 lung cancer, 26 breast cancer, 25 ovarian cancer, 8 kidney cancer, 3 gastric cancer and 2 hepatic cancer, were included. The mean number of drugs/patient was $7.27(\mathrm{SD} \pm 4.5)$. Adverse events and ADRs were 234 and 223 respectively (105 patients had at least one ADR, range 1-8 ADRs/patient). Among the 223 ADRs, 27 were serious (21 patients had at least one serious ADR, range 1-3 serious ADRs/patient) and $10.31 \%$ were unexpected. The most frequently reported ADRs (at least 5 cases for single ADR) were: cutaneous toxicity $(\mathrm{n}=31)$, diarrhoea $(\mathrm{n}=27)$, neutropenia $(\mathrm{n}=23)$, mucositis $(\mathrm{n}=18)$, nausea $(\mathrm{n}=15)$, hypertension $(\mathrm{n}=15)$, palmar-plantar erythrodysesthesia $(\mathrm{n}=11)$, asthenia $(\mathrm{n}=10)$, hypertransaminasemia $(\mathrm{n}=5)$. ADR outcomes were: resolved (41.29\% of cases), resolving (39.35\%), not resolved $(8.39 \%)$, not specified $(7.09 \%)$ and resolved with sequalae $(3.87 \%)$. The most frequently combinations of suspected drug-ADR were: bevacizumab-neutropenia $(\mathrm{n}=15)$; bevacizumab-nausea $\quad(\mathrm{n}=10)$; bevacizumab-diarrhoea $(\mathrm{n}=6)$; bevacizumab-hypertension $(\mathrm{n}=5)$; cetuximab-cutaneous toxicity $(\mathrm{n}=9)$; erlotinib-cutaneous toxicity $(n=17)$; erlotinib-diarrhoea $(n=5)$; and erlotinib-mucositis $(n=5)$. The identified DDIs were: erlotinib-esomeprazole $(\mathrm{n}=1)$; erlotinib-lansoprazole $(n=13)$; erlotinib-magaldrate $(n=6)$; erlotinib-omeprazole $(\mathrm{n}=6)$; erlotinib-pantoprazole $(\mathrm{n}=7)$; gefitinib-lansoprazole $(\mathrm{n}=1)$; sunitinib-sotalol $(n=2)$. None of these resulted in expected ADRs.

Discussion: The sensitization of drug safety may be improved especially in the oncological setting.

Conclusion: Drug safety monitoring in cancer patients is a priority issue. Healthcare professionals should be adequately stimulated in detection and reporting of ADRs.

Further sources of information/References

1. Wahlang JB, Laishram PD, Brahma DK, Sarkar C, Lahon J, Nongkynrih BS. Adverse drug reactions due to cancer chemotherapy in a tertiary care teaching hospital. Ther Adv Drug Saf 2017;8:61-6

2. Chan A, Soh D, Ko Y, Huang YC, Chiang J. Characteristics of unplanned hospital admissions due to drug-related problems in cancer patients. Support Care Cancer 2014;22:1875-81

3. Tuccori M, Montagnani S, Capogrosso-Sansone A, Mantarro S, Antonioli L, Fornai M, et al. Adverse reactions to oncologic drugs: spontaneous reporting and signal detection. Expert Rev Clin Pharmacol 2015;8:61-75

\section{Comparative Risk of Cause-Specific Mortality in Older Patients Prescribed Codeine or Tramadol for Non-Malignant Pain: Retrospective Cohort Study}

\section{Allen $^{1}$, W. Meeraus ${ }^{1}$, K. Donegan ${ }^{1}$ \\ ${ }^{I}$ Medicines And Healthcare Products Regulatory Agency, London, $U K$}

Introduction: Opioids are increasingly being used to treat non-malignant pain, despite risks of tolerance and dependence [1]. A US insurance claims database study counterintuitively suggested a greater risk of all-cause mortality for codeine, but not tramadol, relative to hydrocodone [2]. We previously reported a similar result in the UK Clinical Practice Research Datalink (CPRD), but this was explained by confounding by age, gender and lifestyle variables [3]. We have subsequently carried our additional analyses to investigate cause-specific mortality using linked mortality data to further explore this signal.

Aim: To compare the risk of cause-specific mortality in adults aged 65+ years prescribed codeine or tramadol for non-malignant pain in UK primary care.

Methods: Data on patients newly prescribed codeine or tramadol between 01/01/1998-10/01/2012 and who were eligible for linkage to death registration data were extracted from the CPRD. Cause-specific death data were obtained through linkage to Office for National Statistics mortality data. Exclusion criteria included: previous opioid/NSAID prescription, malignancy, opioid dependence/illicit drug use in the year prior to the index date. Follow-up was censored upon transfer out of the GP practice, last data collection, date of switch to different opioid, $90+$ day treatment gap, 365 days after the final opioid prescription, death or 10/01/2012 whichever was earliest. All-cause mortality hazard ratios were estimated using the Cox model and cause-specific mortality subhazard ratios using a competing risks model with alternative causes of death treated as competing risk events. Hydrocodone is not available in the UK, thus tramadol was compared directly with codeine. 
Results: 75,468 CPRD patients were eligible for linkage to the ONS mortality data. 39,882 patients were prescribed codeine (mean age 75.8 years, $38.0 \%$ male) and 35,586 tramadol (74.9 years, $38.6 \%$ male). The most common indications for use were for back pain (14.7\%) and fractures (19.4\%). After adjustment for potential confounding, there was no evidence of an association for all-cause mortality for tramadol relative to codeine. We found no association for the three most common causes of death in both unadjusted and adjusted models (table 1).

Conclusions: The unadjusted HR for all-cause mortality is consistent with the US study, albeit with a different comparator [2]. However, after adjustment for confounding, the association for all-cause mortality was significantly attenuated. We found no evidence of an association for deaths from cancer, circulatory or respiratory disease. Overall, this study provides reassuring data regarding the relative safety of codeine and tramadol in this patient population.

Further sources of information/References

1. Zin CS, Chen LC, Knaggs RD. Changes in trends and pattern of strong opioid prescribing in primary care. Eur J Pain 2014; 18:1319-51

2. Solomon DH, Rassen JA, Glynn RJ, et al. The comparative safety of opioids for nonmalignant pain in older adults. Arch Intern Med 2010; 13;170:1976-86

3. Allen C, Meeraus W, Donegan K. Comparative Risk of All-Cause Mortality in Older Patients Prescribed Codeine Or Tramadol for NonMalignant Pain: Retrospective Observational Cohort Study. Pharamcoepidemiol Drug Saf 2016; 25(Suppl. 3): 368

Table 1. Hazard (HR) and subhazard (sHR) ratios for mortality (tramadol versus codeine)
\begin{tabular}{|l|l|l|l|}
\hline Cause of death & Number of deaths & Unadjusted model (95\% Cl) & Adjusted model $(95 \% \mathrm{Cl})$ \\
\hline All causes & 5,338 & $\mathrm{HR}=0.91(0.86-0.96) p<0.001$ & $\mathrm{HR}=0.99(0.94-1.04) \mathrm{p}=0.680$ \\
\hline Cancers & 1,315 & $\mathrm{sHR}=1.03(0.93-1.15) \mathrm{p}=0.570$ & $\mathrm{sHR}=1.09(0.91-1.22) \mathrm{p}=0.104$ \\
\hline $\begin{array}{l}\text { Circulatory } \\
\text { disease }\end{array}$ & 1,936 & $\mathrm{sHR}=0.98(0.89-1.07) \mathrm{p}=0.586$ & $\mathrm{sHR}=1.09(0.99-1.19) \mathrm{p}=0.070$ \\
\hline $\begin{array}{l}\text { Respiratory } \\
\text { disease }\end{array}$ & 899 & $\mathrm{sHR}=0.90(0.79-1.03) \mathrm{p}=0.140$ & $\mathrm{sHR}=0.99(0.87-1.13) \mathrm{p}=0.912$ \\
\hline
\end{tabular}

\section{An Analysis of Spontaneous Reports on Adverse Drug Reactions in Child and Adolescent Psychiatry in Germany}

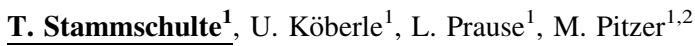 \\ ${ }^{1}$ Drug Commission of the German Medical Association, Berlin, \\ Germany, ${ }^{2}$ Department for Child and Adolescent Psychiatry, \\ Municipal Hospital Karlsruhe, Karlsruhe, Germany
}

Background: The use of medicines in child and adolescent psychiatry is increasing and thus a higher number of adverse drug reactions (ADRs) can be assumed. Besides vaccines, methylphenidate and atomoxetine induce the highest number of ADR reports in children [1] and off-label use of antipsychotics and antidepressants in this age group may cause safety problems.

Aim: To identify potential safety signals for medicines used for psychiatric indications in children and adolescents.

Methods: Reports in the German spontaneous reporting database with antipsychotics, antidepressants or stimulants as suspected medicines that refer to children and adolescents were included. Besides a descriptive analysis we screened for signals by identifying reactions with $\geq$ reports that are not labelled in the corresponding product information. These potential signals were matched with published reports in PubMed and VigiLyze ${ }^{\circledR}$.
Results: In approximately $13,400(4.2 \%)$ of 320,000 reports (1990 to August 2016) referring to small molecule drugs, the affected patients were between the ages of 3 to 18 . The proportion of reports with suspected psychoactive medicines in this age group was about $20 \%$ and has increased since the late 1990s. Most frequently suspected were stimulants (41\%), followed by antipsychotics (36\%), and antidepressants (23\%). The three most frequently suspected substances in each group were risperidone, clozapine, aripiprazol, fluoxetine, citalopram, venlafaxin and methylphenidate, atomoxetin and lisdexamfetamin, respectively. Citalopram and venlafaxin are not approved for this age group. Potential signals were identified for the substance with the highest total number of reports, methylphenidate $(\mathrm{n}=930)$ and for lisdexamfetamin $(\mathrm{n}=92)$ (Table 1$)$.

Table 1 The table conveys identified potential signals, defined as $\geq 3$ reports of adverse reactions not labelled in the corresponding product information, and the results of a research for equivalent reports in PubMed and VigiLyze (WHO database of spontaneous reports)

\begin{tabular}{|l|l|l|l|}
\hline Substance & $\begin{array}{l}\text { Potential signal } \\
\text { (No. of reports) }\end{array}$ & $\begin{array}{l}\text { Matching literature } \\
\text { reports }\end{array}$ & $\begin{array}{l}\text { Positive IC value in } \\
\text { VigiLyze }\end{array}$ \\
\hline \multirow{5}{*}{ Methylphenidate } & Priapism* & + & + \\
\cline { 2 - 4 } & Qt-elongation & + & + \\
\cline { 2 - 4 } & Speech disorder & + & + \\
\cline { 2 - 4 } & Thrombocytosis & + & + \\
\cline { 2 - 4 } & Compulsive symptoms & + & + \\
\hline Lisdexamfetamin & Compulsive symptoms & + & \\
\hline
\end{tabular}

Conclusions: By using a basic methodology we revealed potential safety signals for methylphenidate and lisdexamfetamine which should be further evaluated by other methods. Some of the most frequently reported medicines were used off-label in this age group.

Further sources of information/References

1. Hawcutt D. et al. Reported paediatric adverse drug reactions in the UK 2000-2009. Br J Clin Pharmacol 2011; 73: 437-46

\section{Spontaneous reporting of adverse drug reactions associated with newer long-acting injectable antipsychotics in Italy}

P. Maria Cutroneo ${ }^{1}$, M. Antonietta Barbieri ${ }^{2}$, G. Cicala ${ }^{2}$,

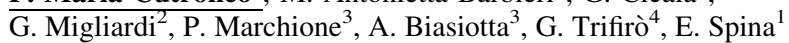

${ }^{1}$ Sicilian Regional Pharmacovigilance Centre, Messina, Italy, ${ }^{2}$ Dept. of Clinical and Experimental Medicine, University of Messina, Messina, Italy, ${ }^{3}$ Italian Medicines Agency, Pharmacovigilance Office, Rome, Italy, ${ }^{4}$ Dept. of Biomedical and Dental Sciences and Morphofunctional Imaging, University of Messina, Messina, Italy

Background: Long-acting injectable (LAI) antipsychotics are effective in the long-term management of schizophrenia, reducing the risk of relapse and rehospitalization due to nonadherence. Since the introduction of risperidone LAI in 2006, three additional second-generation antipsychotics have become available in long-acting injectable formulations: olanzapine pamoate (2010), paliperidone palmitate (2012), and C LAI (2014). Safety profiles of LAI antipsychotics seem to be consistent with their oral parent formulations, even though unexpected safety signals were occasionally observed in clinical practice.

Aim: to describe adverse drug reactions (ADR) associated with newer LAI antipsychotics based on the Italian spontaneous reporting system (SRS) database.

Methods: We performed an analysis of the Italian SRS database, selecting all the ADR reports attributed to second-generation LAI antipsychotic drugs from their launching to December 2016. We described the frequency 
of compound-specific ADRs by MedDRA system organ class (SOC) and preferred terms (PTs).

Results: Over a 10-year period (2006-2016), 304 reports of ADRs related to second-generation LAI antipsychotics were identified in the Italian SRS database. The mean age $( \pm \mathrm{SD})$ of LAI antipsychotic users was 42.8 $( \pm 13.8)$ years. The spontaneous reports regarded more commonly males $(\mathrm{n}=175 ; 57.6 \%)$ than females $(\mathrm{n}=129 ; 42.4 \%)$. Serious ADRs accounted for $38.2 \%(n=116)$ of total second-generation LAIs-related reports. Paliperidone palmitate was associated with the highest number of reports $(\mathrm{n}=97 ; 31.9 \%)$, followed by olanzapine pamoate $(\mathrm{n}=86 ; 28.3 \%)$, risperidone LAI $(n=63 ; 20.7 \%)$ and aripiprazole LAI $(n=58 ; 19.1 \%)$. The most represented ADRs involved central nervous system, such as sedation $(n=61)$, extrapyramidal symptoms $(n=38)$ and neuroleptic malignant syndrome $(n=6)$, followed by hyperprolactinemia $(n=31)$ and weight increase $(\mathrm{n}=19)$. The rate of extrapyramidal effects on total ADRs reports for each drug was $23.7 \%$ with paliperidone palmitate, $20.6 \%$ with risperidone LAI, $17.2 \%$ with aripiprazole LAI and $8.1 \%$ with olanzapine pamoate. Hyperprolactinemia was mainly related to risperidone LAI $(20.6 \%)$ and paliperidone palmitate (16.5\%), while weight increase was most reported in association with aripiprazole LAI (10.3\%). Co-occurrence of excessive sedation, confusion and disorientation, occurring within few hours after injection and sometimes resulting in coma was described in 15 ADR reports related to olanzapine pamoate. These symptoms are consistent with the potentially serious ADR known as post-injection delirium/sedation syndrome.

Conclusions: Our analysis shows that the tolerability and safety profile of second-generation LAI antipsychotics differ among the available agents and is substantially similar to the corresponding oral formulations. However, some cases of post-injection delirium/sedation syndrome were associated with olanzapine pamoate, confirming the need for a 3-hours post-injection observation period.

\section{Bisphosphonate-Induced Carpal Tunnel Syndrome. A disproportionality Analysis in VigiBase}

\author{
A. Carvajal $^{1}$, F. Antonio Lizaroso-Soto ${ }^{2}$, T. Oscanoa ${ }^{2,3}$ \\ ${ }^{1}$ Universidad de Valladolid, Valladolid, Spain, ${ }^{2}$ Instituto de \\ Investigación de la Facultad de Medicina de la Universidad de San \\ Martin de Porres, Lima, Peru, ${ }^{3}$ Centro de Investigación de Seguridad \\ de Medicamentos de la Facultad de Medicina de la Universidad de \\ San Martin de Porres, Departamento de Medicina del Hospital \\ Almenara, Lima, Peru
}

Introduction: Bisphosphonates have proven to avoid osteoporotic fractures, and are widely used to prevent these fractures, particularly in postmenopausal women. However, adverse reactions may appear- commonly gastric inflammation and oesophageal erosions, and raised risk of musculoskeletal pain; more rarely, osteonecrosis of the jaw and conflicting evidence regarding risk of oesophageal cancer have also been described. Additionally, cases of synovitis, a well-recognized cause of carpal tunnel syndrome (CTS), have occasionally been reported [1]; recently, an observational study has identified an association between bisphosphonates and CTS [2]. CTS is the commonest peripheral nerve condition and has considerable employment and healthcare costs; it is caused by elevated pressure in the carpal tunnel, and this in turn produces compression of the median nerve, resulting in impaired nerve conduction, paraesthesia and pain.

Aim: To further learn upon the association between bisphosphonates and CPT.
Methods: A search was conducted by using VigiLyze, which provides access to more than 14 million Individual Case Safety Reports in VigiBase, a World Health Organization's database; these reports are submitted through National Pharmacovigilance Centers by more than 100 countries around the world. For the purpose, exposure was defined as the mention of a bisphosphonate (alendronic acid) in a report, either being or not being suspected of causing the reaction; the remaining reports were non-exposure, and the outcome of interest was CTS. The association between bisphosphonates and CPT was estimated by means of the Information Component (IC) [2]. IC relates observed and expected values to find drugadverse effect combinations; an IC above 0 is considered as an association. Results: In the database, there were 5829 cases of CPT up to May 2017; the total number of reports was $14,873,497$ by that date. The overall estimate in the database was 4 cases of CPT every 100,000 reported cases of whatever condition. Among bisphosphonates, alendronic acid concentrates most of the cases; out of 44,513 total cases reported for this drug, 356 were cases of CTS ( 80 per 100,000). The IC estimate was 4.31 (IC025, 4.16). Most cases referred to elderly people and women (women, 291; men, 11; unknown, 54). Alendronic acid was the sole suspected drug in 226 cases; positive de-challenge or re-challenge occurred in 7 and 1 cases, respectively; most cases were considered as serious (350).

Conclusions: The present estimates along with evidence from other sources are consistent with a causal relationship between bisphosphonate exposure and the appearance of CTS

\section{Further sources of information/References}

1. Jones DG, Savage R, Highton J. Synovitis induced by alendronic acid can present as acute carpal tunnel syndrome. BMJ 2005; 330:74

2. Carvajal A, Martín Arias LH, Sáinz M, Escudero A, Fierro I, Sauzet O,Cornelius VR, Molokhia M. Carpal Tunnel Syndrome Associated with OralBisphosphonates. A Population-Based Cohort Study. PLoS One 2016;11: e0146772

3. Bate A, Lindquist M, Edwards IR, Olsson S, Orre R, Lansner A, De Freitas RM. A Bayesian neural network method for adverse drug reaction signal generation. Eur J ClinPharmacol 1998; 54:315-21

\section{Data Management in Pharmacovigilance: Challenges and complexities}

\section{Chopra ${ }^{1}$ \\ ${ }^{1}$ Apcer Life Sciences, New Delhi, India}

Background: Pharmacovigilance is a highly sensitive field involving monitoring of the safety of drugs and taking action to reduce risk. Pharmacovigilance relies on information gathered from the collection of individual case safety reports and other pharmacoepidemiological data. A poor pharmacovigilance data management not only jeopardizes patient safety, it also increases the risk of investing in the development of wrong product which causes a huge loss to a pharmaceutical company. Therefore, it is very important to establish a robust pharmacovigilance data management system which complies with the stringent regulatory guidelines, global pharmaceutical norms and ultimately safeguard the pharmacovigilance.

Objective:

To overcome the challenges in managing case processing in Pharmacovigilance?

To resolve the technical issues related to the coding of events and drugs? To manage the regulatory compliance successfully?

Method: A review of literature was done to identify the challenges and complexities with regards to the data management in pharmacovigilance. The following approach was followed to conduct the literature search: 
- A detailed review of the literature to understand the complexities and challenges faced by small, mid-sized and large pharma companies with regards to the data management

- Comparative Analysis to understand the challenges in data management with regards to clinical trial PV and post marketing PV.

Results: Drug Safety is a highly sensitive issue and therefore it is imperative to have robust data management practices in any Pharmacovigilance Department. A very intensive team effort is required for the successful outcome in the data management operations in Pharmacovigilance. Many steps are involved in the processing of individual adverse event/ suspected adverse reaction report cases, all requiring high and complex degrees of technical skill and judgment to ensure that the information is properly documented, reviewed, understood, and placed in proper perspective relative to a developing/or already established benefitrisk profile for the product. The session will cover some of the important topics like source of various reports, Triage of cases, Individual case safety report processing in Safety data base for Clinical trial and marketed products (Data entry, QC review, medical review), application of MedDRA and WHO-DD dictionaries, SAE reconciliation activities and use of various types of Safety data bases e.g. Argus, Aris G, Vigiflow and Oracle AERS.

Conclusion Pharmacovigilance continues to play a crucial role in meeting the challenges posed by the ever increasing range and potency of drugs, all of which carry an inevitable and sometimes unpredictable potential for harm. The harm can be minimized by ensuring that drugs of good quality, safety and efficacy are used rationally, and that the expectations and concerns of the patient are taken into account when therapeutic decisions are made. Good quality data is thus imperative to achieve the desired results.Functioning, well documented, and transparent quality management systems benefit not only those involved in data collection, management and output production, but, ultimately, the patients.

\section{Effectiveness of Clinical Pharmacist Review of Direct Oral Anticoagulants Prescriptions and Scope of Identified Errors}

\author{
O. Lavon ${ }^{1}$, M. Salameh ${ }^{2}$, H. Makhoul ${ }^{2}$, T. Lavi ${ }^{2}$, L. Zack ${ }^{2}$ \\ ${ }^{1}$ Clinical Pharmacology and Toxicology Unit, Carmel Medical \\ Center, Haifa, Israel, ${ }^{2}$ Pharmacy Services, Carmel Medical Center, \\ Haifa, Israel
}

Background: Anticoagulants use is related to significant risk for serious adverse events. The direct oral anticoagulants (DOACs) are relatively new in clinical practice and physicians' learning curves of good prescription practice of DOACs may lead to medications errors. To mitigate the potential risk of DOAC prescription errors a mandatory review by clinical pharmacists prior to medication dispensing was implemented in Carmel Medical Center, Haifa, Israel.

Aim: To evaluate the effectiveness of the clinical pharmacist mandatory review of DOAC prescriptions and the scope of identified errors.

Methods: A single medical center retrospective review of all DOAC prescriptions reviewed by clinical pharmacists between January 1, 2012 to December 31, 2016. Primary outcome was percentage of inappropriate and erroneous prescriptions. Secondary outcomes were percentage of prescription errors per DOAC, types for errors and pharmacist interventions. Results: During the study period 2612 DOAC prescriptions were reviewed by clinical pharmacists, of which $530(20.3 \%)$ were inappropriate or erroneous. There were no significant differences in the percentage of prescription errors between the different DOACs. Main types of the errors were contraindications for DOACs due to reduced renal functions or presence of a significant mitral disease and inappropriate dose. Pharmacist interventions included dose correction (50\%), recommendation for avoiding any DOACs (35.7\%) and anticoagulant substitution (14.3\%). Conclusions: The clinical pharmacist mandatory review of DOAC prescriptions was effective in identifying and preventing a significant number of errors. It is advised to implement a pharmacist review of anticoagulants or any high-alert medication as an effective measure to prevent medication errors.

\section{Epidemiological and clinical profile of adverse effects of antineoplastic agents, Pharmacovigilance in oncology, Morocco, 2012-2016}

\author{
I. Talibi $\mathbf{i}^{\mathbf{1 , 2 , 3}}$, E. Elkarimi ${ }^{3}$, B. Meddah ${ }^{5}$, H. Errihani ${ }^{4,5}$, \\ H. Bechar ${ }^{4}$, H. Sefiani ${ }^{3}$, A. El Fassi Fihri' ${ }^{4}$, L. Alj ${ }^{1,2,3}$, I. Smaili $^{2,3}$, \\ A. Tebaa ${ }^{3}$, A. Khattabi ${ }^{1,2}$, R. Soulaymani-Bencheikh ${ }^{3,5}$ \\ ${ }^{1}$ Ecole Nationale de Santé Publique, Rabat, Morocco, ${ }^{2}$ Field \\ Epidemiology Training Program, Rabat, Morocco, ${ }^{3}$ Centre Anti \\ poison et de Pharmacovigilance du Maroc, Rabat, Morocco, ${ }^{4}$ Institut \\ National d'Oncologie, Rabat, Morocco, ${ }^{5}$ Université Mohammed V, \\ Faculté de Médecine et de Pharmacie, Rabat, Morocco
}

Background: Cancers are the leading cause of morbidity and mortality worldwide. In 2012, there were 14 million new cases and 8.2 million cancer deaths. In Morocco, cancer causes an estimated 35,000 new cases and 22800 deaths annually. Chemotherapy involves highly complex regimens and hence accounts to high susceptibility toward adverse drug reactions (ADRs). Antineoplastic drugs are one of the main causes of drug-induced events. The Centre Anti poison et de Pharmacovigilance of Morocco (CAPM) created a specific unit for pharmacovigilance of drugs used in oncology in 2014 to analyze their severity, frequency and preventability. The outcome would create awareness among health care providers and patients, and implement measures to minimize risks.

Objective: The purpose of this study is to analyze the pattern of adverse drug reactions (ADRs) to antineoplastic agents in cancer patients reported to the CAPM.

Methods: We conducted a retrospective observational study of the ADRs reported to the CAPM for the five years period, January 2012 to December 2016. We used the Anatomical Therapeutic Chemical classification code system for drugs, and WHO Adverse Drug Reaction Terminology for adverse drug reactions, whereas the seriousness of those events followed the ICH E2A guidelines. These reports were analyzed for various carcinomas under treatment, medications used, types of ADRs, organ system involvement, seriousness. We carried out frequencies and other descriptive statistics by using Excel 2010 in combination with Uppsala Monitoring Center-VigiLyze $^{\mathrm{TM}}$ software.

Results: During the period of our study, 1510 cases of adverse effects under antineoplastic agents were reported. The sex ratio $\mathrm{M} / \mathrm{F}$ was 0.35 and the mean age $50( \pm 16)$ years. The main reported drugs were Docetaxel (14.4\%), Imatinib (14.1\%), Trastuzumab (10.8\%) and Capecitabine $(8.9 \%)$. Breast cancer was the most frequent indication $(27.4 \%)$. The most reported ADRs were: skin disorders (24.6\%), blood disorders (21.3\%) and gastrointestinal disorders (18.6\%). When seriousness is indicated, 56\% ADRs were considered serious.

Conclusion: This study identified the frequently reported antineoplastic agents in the occurrence of adverse reactions in Morocco and their natures. It demonstrated the high proportion of serious ADRs with antineoplastic agents as well. As limit for our study, the outcome of ADRs was not specified in $46.8 \%$. We recommend using this data for the development of 
risk management plans and put into place effective measures to reduce the burden of ADRs and thereby further improve the benefit.

\section{Guillain-Barré Syndrome associated with vaccines in children: a case-control study}

\section{F. Menniti-Ippolito ${ }^{1}$, R. Da Cas ${ }^{1}$, R. Rossi ${ }^{2}$, U. Raucci ${ }^{2}$,} and the Multicenter Study Group on Drug and Vaccine Safety in Children

${ }^{1}$ Italian National Institute Of Health, Rome, Italy, ${ }^{2}$ Bambino Gesù Pediatric Hospital, Rome, Italy

Introduction: Guillain-Barré Syndrome (GBS) is an uncommon disorder characterized by an acute acquired immune-mediated polyradicoloneuropathy with crude incidence ranging from 0.81 (Canada) to 1.89 (Italy) cases per 100,000 person-years. The etiology of GBS is not yet completely understood, but several events have been observed which occur before development of disease including acute infections and immunizations. Several case studies and epidemiologic studies reported a relation between GBS and different vaccines. Nevertheless, a previous case-control study and a recent large retrospective study did not find evidence of an increased risk of GBS following vaccinations of any kind, including influenza vaccination $[1,2]$.

Aim: To estimate possible association between vaccination and GBS.

Methods: An active surveillance on drug and vaccine safety in pediatrics (age 2 months-17 years) is ongoing since 1999 in 11 clinical centers in Italy. All children, hospitalized for selected acute clinical conditions of interest through the local Paediatric Emergency Department, are enrolled in the study. Data on drug and vaccine use in children before the onset of symptoms leading to hospitalization are collected by parent interview. A case-control design is applied for risk estimates: exposure in children with GBS, included as cases, with similar exposure in children with gastroduodenal lesions, enrolled as controls.

Results: Up to May 201739 cases of GBS and 758 controls were identified. The median age was 4 years in cases (range $8 \mathrm{~m}-14$ y) and 3 years (range $1 \mathrm{~m}-20 \mathrm{y}$ ) in controls. Females were $52 \%$ in the GBS group (cases) and $45 \%$ in the gastroduodenal group (controls). The estimated crude OR of GBS associated with vaccination was 0.85 (95\% CI 0.21-2.45).

Conclusions: No risk of vaccination in GBS occurrence was estimated in this study. This multicenter study represents an useful tool to point out in relatively short time possible risk signals of drugs and vaccines used in children. It can be used to confirm or confute risks emerging from the literature associated with new drugs or vaccines or signals emerging from spontaneous reporting systems.

\section{Further sources of information/References}

1. Liu GF,Wu ZL,Wu HS,Wang QY, Zhao-RiGT,Wang CY, et al. A case-control study on children with Guillain-Barre syndrome in North China. Biomed Environ Sci 2003;16:105-11

2. Baxter R, Bakshi N, Fireman B, et al. Lack of association of vaccinations with Guillain-Barré syndrome. Clin Infect Dis 2013;57 : 197-204
146 Influencing Adverse Drug Reaction Reporting In Primary Care

\author{
C. Anton ${ }^{1}$, A. Dussaye ${ }^{2}$, K. Badyal ${ }^{1}$, R. Ferner ${ }^{1,2}$ \\ ${ }^{1}$ Yellow Card Centre West Midlands, City Hospital, Birmingham, UK, \\ ${ }^{2}$ Institute of Clinical and Experimental Medicine, University \\ of Birmingham, $U K$
}

Background: The Yellow Card Scheme (YCS) is well established as an integral component of pharmacovigilance in the UK. However underreporting of suspected ADRs is a weakness of any spontaneous scheme [1]. An education intervention to promote awareness of the Yellow Card scheme can increase reporting [2].

Objective: To evaluate the impact on the ADR reporting rates of healthcare professionals of an educational intervention about the Yellow Card Scheme and gain an insight into the main issues that clinicians face regarding pharmacovigilance

Methods: We compared 2 clusters of GP surgeries, and 2 clusters of community pharmacies in our region. All had poor baseline reporting rates. The clusters received a link to a SurveyMonkey questionnaire to test their knowledge of pharmacovigilance and in one of each pair we followed this with a brief educational leaflet about the Yellow Card scheme.

Results: 21 questionnaires were completed out of a total of 625 sent to healthcare professionals in the 4 areas. Most common responses to some of the questions are set out in the Table below

Table: Questionnaire responses
\begin{tabular}{|l|l|}
\hline Question Subject & Most common answers \\
\hline No of YCs completed in previous 12 months & $\begin{array}{l}0(57 \% \text { of respondents) } \\
1 \text { or } 2(33 \%)\end{array}$ \\
\hline Meaning of the black triangle symbol & Correct $(81 \%)$ \\
\hline Factors discouraging reporting & Lack of time $(55 \%)$ \\
\hline Methods to increase reporting & $\begin{array}{l}\text { Monthly newsletter detailing case report (38\%) } \\
\text { Faster, easier method of reporting (38\%) }\end{array}$ \\
\hline
\end{tabular}

There was an increase in the number of Yellow Cards received from the areas which received the survey from 0.75 reports per month from GPs to 2.5 reports per month (compared with a regional average change of 22.6 reports per month to 20.5 reports per month) but no difference between the areas that received the educational leaflet and those that did not. There was no change in the number of community pharmacy reports received.

Conclusion: Survey Monkey questionnaires elicited few responses from poor reporters but may have stimulated reporting as there was a $300 \%$ in reporting. The brief educational leaflet had no additional effect.

Discussion: The trial did not support the hypothesis that an additional intervention would encourage poor reporters. We were unable to be sure that an invitation to participate in a survey had an effect, as we had only historical controls. From the responses we obtained, healthcare professionals would welcome faster ways of reporting, but roll-out of Yellow Card reports integrated into e-prescribing systems has been very slow in our Region.

Further sources of information/References:

1. Jamie J Coleman and Sarah K Pontefract. Adverse drug reactions. Clin Med 2016; 16: 481-5 
2. Lopez-Gonzalez E et al. Effect of an educational intervention to improve adverse drug reaction reporting in physicians: a cluster randomized controlled trial. Drug Saf. 2015; 38: 189-96

\section{Muscular adverse drug reactions associated with proton pump inhibitors: a disproportionality analysis using the Italian National Network of Pharmacovigilance database}

I. Convertino ${ }^{1}$, A. Capogrosso Sansone ${ }^{1}$, M. Galiulo ${ }^{1}, \mathrm{~S}$. Salvadori $^{2}$, S. Pieroni ${ }^{2}$, T. Knezevic ${ }^{2}$, S. Mantarro ${ }^{1}$, A. Marino ${ }^{1}$, M. Hauben ${ }^{3}$, C. Blandizzi ${ }^{1,4}$, M. Tuccori ${ }^{4}$

${ }^{1}$ Division of Pharmacology and Pharmacovigilance, Department of Clinical and Experimental Medicine, University of Pisa, Pisa, Italy, ${ }^{2}$ Institute of Clinical Physiology, National Research Council, Pisa, Italy, ${ }^{3}$ Pfizer Inc., New York, USA, ${ }^{4}$ Unit of Adverse Drug Reactions Monitoring, University Hospital of Pisa, Pisa, Italy

Introduction: After cerivastatin marketing withdrawal for rhabdomyolysis's fatal cases [1], statins represent the drug class most commonly associated with muscular adverse drug reactions (ADRs) [2]. However, also proton pump inhibitors (PPIs) have been related to myopathies [3]. Unfortunately, these potential signals may be under-detected due to the statins masking effect.

Aim: To assess the reporting risk of muscular ADRs with PPIs on spontaneous reports in the Italian Pharmacovigilance Network database.

Methods: A case/non-case analysis on data from July 1983 to May 2016 was performed. Published case reports, reports on adverse events following immunization, low-quality reports and duplicate reports were excluded [4]. Cases were identified by reports containing at least one muscular ADR. Non-cases were all reports containing ADRs other than muscular ones. In the primary analysis, reports with at least one suspected PPI were classified as index group while all other reports were the reference. Reporting odds ratio (ROR) and 95\% confidence intervals (CIs) were calculated [5]. A sub-analysis only on rhabdomyolysis reports in the cases group was performed. In a secondary and tertiary analysis, we explored the association of PPIs with muscular ADRs after removing the statins masking effect [6]. The unmasking was performed by excluding reports with suspected statins and by including cases with at least one PPI (both suspected and concomitant). Moreover, a possible PPIs-statins interaction was also tested. The RORs were adjusted for age, gender, number of drugs and thyroid diseases by logistic regression.

Results: Overall 274,108 reports were analysed. In the primary analysis, the RORs of muscular ADRs for PPIs, adjusted for age and gender, was 1.484 (95\% CI 1.204-1.829; $\mathrm{p}<0.001$ ), whereas the adjusted ROR for rhabdomyolysis was 0.621 (95\% CI $0.258-1.499)$. In the secondary analysis, similar results were obtained (adjusted ROR 1.200; 95\% CI 0.447-3.224). A potential association of rhabdomyolysis-PPIs was detected in the tertiary analysis, where PPIs were considered independently from their role (adjusted ROR: 1.667, 95\% CI 1.173-2.369; $\mathrm{p}<0.01)$. No potential signal with PPI-statin interaction and muscular ADR/rhabdomyolysis was observed.

Discussion: RORs for rhabdomyolysis did not reach the minimum criteria for signal detection. Only in the tertiary analysis, the rhabdomyolysis frequency related to PPIs was higher than any other ADRs, when compared to reports not including PPIs or statins. However, this finding should be confirmed by further investigations.
Conclusion: A potential signal of disproportionate reporting for muscular ADRs related to the whole PPI class was identified and this was enhanced after the unmasking.

Further sources of information/References

1. Staffa JA, Chang J, Green L. Cerivastatin and reports of fatal rhabdomyolysis. New Engl J Med 2002;346:539-40

2. Conforti A, Chiamulera C, Moretti U, Colcera S, Fumagalli G, Leone R. Musculoskeletal adverse drug reactions: a review of literature and data from ADR spontaneous reporting databases.Curr Drug Saf 2007;2:47-63

3. Clark DW, Strandell J. Myopathy including polymyositis: a likely class adverse effect of proton pump inhibitors? Eur J Clin Pharmacol 2006;62:473-9

4. Yue Z, Shi J, Jiang P, Sun H. Acute kidney injury during concomitant use of valacyclovir and loxoprofen: detecting drug-drug interactions in a spontaneous reporting system. Pharmacoepidemiol Drug Saf 2014;23:1154-9

5. Piccinni C, Gissi DB, Gabusi A, Montebugnoli L, Poluzzi E. Paraesthesia after local anaesthetics: an analysis of reports to the FDA Adverse Event Reporting System. Basic Clin Pharmacol Toxicol 2015;117:52-6

6. Maignen F, Hauben M, Hung E, Van Holle L, Dogne JM. Assessing the extent and impact of the masking effect of disproportionality analyses on two spontaneous reporting systems databases. Pharmacoepidemiol Drug Saf 2014;23:195-207

\section{Does the Use of Vancomycin is Efficient and Safety in Renal Failure Patients?}

I. Salouage ${ }^{1,2}$, N. Jebabli ${ }^{1,2}$, E. Gaïes ${ }^{1,2}$, R. Charfy ${ }^{1,2}$, H. El

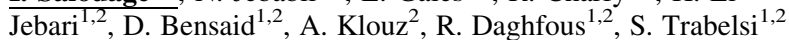

${ }^{1}$ Service de Pharmacologie Clinique. National Centre of Pharmacovigilance of Tunisia, Tunisie., Tunis, Tunisia,

${ }^{2}$ Laboratoire de recherche de pharmacologie clinique et expérimentale, Tunis, Tunisia

Background: Vancomycin (VCM) is a glycopeptide antibiotic generally used for the treatment of gram-positive infections. Therapeutic drug monitoring (TDM) of VCM is essential because of its pharmacokinetics variability in particular in renal failure. Several regimens are used in renal failure patients to adjust VCM dosage.

The aim of this study was to evaluate the TDM of VCM in patients with renal failure and to evaluate the correlation between received and estimated dose of VCM.

Methods: We achieved a retrospective study in the National Centre of Pharmacovigilance. Trough plasma concentrations of VCM were performed by fluorescence polarization immunoassay. The therapeutic range was 15 to $20 \mu \mathrm{g} / \mathrm{mL}$. Cockroft formula was used to evaluate the degree of renal failure. The estimated dose was calculated using this formula $[($ Clearance of creatinine $* 15)+150]$.

Results: The number of samples was 585. The mean trough plasma concentration was $29.84 \mu \mathrm{g} / \mathrm{mL}$ ( 0.88 to $118 \mu \mathrm{g} / \mathrm{mL})$. $86.5 \%$ of plasma concentrations were outside the therapeutic range. Received dose of VCM varied between 71.42 to $6000 \mathrm{mg} /$ day (median $=1000 \mathrm{mg} /$ day). There was no correlation between received dose and plasma levels of VCM. Estimated dose varied between 150 to $2320.5 \mathrm{mg} /$ day (median $=361.57$ $\mathrm{mg} /$ day) with a significant difference between received and estimated dose of VCM $(\mathrm{P}<0.05)$. 
This study confirms that TDM of VCM is recommended to ensure efficiency and safety in renal failure patients and that dose adjustment must be related to renal failure degree. So, it is important to complete this work by a Bayesian pharmacokinetic approach for predicting VCM concentrations to individualize its dosing regimen.

\section{Funding for Neglected Tropical Diseases to boost Pharmacovigilance staff capacity in Low and Middle Income Countries}

\section{J. SLiwono Yana ${ }^{1}$ \\ ${ }^{I}$ National Pharmacovigilance Centre, Kinshasa, Democratic Republic of the Congo)}

Background: One of the great challenges for the progress of Pharmacovigilance activities in developing countries, especially in Sub-Saharan Africa is the Pharmacovigilance funding gap through national governments, leading to the lack of training and opportunities to develop knowledge and skills to deal with medicines safety monitoring; Existing funding for Neglected tropical diseases should also support Pharmacovigilance activities by strengthening the capacity of the staff and raising awareness among stakeholders on the ground.

Objective/Aim: To identify the existing funding sources to finance the training programmes for the low income countries specially the National Pharmacovigilance Centre staff in D.R. Congo.

Methods: 2 National Pharmacovigilance Centre/D.R. Congo staff applied at the joint EDCTP (European \& Developing Countries Clinical Trials Partnership)-TDR (Special Programme hosted by WHO for Research and Training in Tropical Diseases) Clinical Research and Development Fellowships 2016-2017. The purpose is to support research teams from Low and Middle Income Countries involved in Neglected Tropical Diseases research to acquire specific skills in clinical research and development through placements in pharmaceutical companies, product development partnerships (PDPs) and research institutions.

Results: Both CNPV staff have been selected and are in placements for a duration of 12 months in a Research Institution in Switzerland and a Pharmaceutical Company in Belgium, respectively in the fields of antimalarial safety and vaccine development (safety and efficacy evaluation). Conclusion: The lack of national governments funding should put the brakes on the progress of Pharmacovigilance activities in developing countries. Alternative financial and technical assistance sources are out there and should be utilized.

Discussion: All National Pharmacovigilance Centres throughout the developing countries are not located in university institutions. Therefore, they do not feel concerned enough by these kinds of calls, which may be a significant source of support for Pharmacovigilance activities.

However, it's possible for all Pharmacovigilance centres of Low and Middle Income Countries, including those that are not particularly dedicated to research, to conduct the safety monitoring of Neglected Tropical Diseases medicines in their regions (malaria, VIH/AIDS, tuberculosis, trypanomiasis, filariasis...) either passively or actively. That is one of the requirements for eligibility to these kinds of grants.

\section{Robustness of the CPA-PRR Method in Pharmacovigilance detection}

\author{
N. Trinh ${ }^{1,2}$, E. Sole ${ }^{2}$, M. Benkebil ${ }^{2}$
}

${ }^{1}$ Inserm UMR 1153, Obstetrical, Perinatal and Pediatric Epidemiology Research Team, Research Center for Epidemiology and Biostatistics Sorbonne Paris Cité, Paris Descartes University, Paris, France, ${ }^{2}$ Agence nationale de sécurité du médicament et des produits de santé, Adverse Events and incidents DepartmentSurveillance Division, Saint Denis, France

Background: Pharmacovigilance signal detection commonly utilizes time-invariant disproportionality analysis methods and rarely capitalizes on time dependency [1,2]. The time-series analysis tool, change-point analysis (CPA), is a powerful method to analyze surveillance data $[3,4]$. CPA was applied for the first time in Pharmacovigilance and demonstrate is interest by combining with dynamic Proportional Reporting Ratio (PRR) [5]. The signaling criteria in the CPA-PRR method defines a substance-event as a potential signal when the two following criteria are fulfilled: PRR- is greater than 1 with at least 5 cases, the time series analysis with CPA detects at least two successive change points of PRRand increasing values of those detected points [5].

Objectives: To evaluate the robustness of CPA-PRR method for optimizing pharmacovigilance signal detection.

Methods: We conducted a retrospective analysis using Eudravigilance Database. Test cases were randomly chosen from the signals validated by Pharmacovigilance Risk Assessment Committee (PRAC) at the European Medicine Agency: Ustekinumab-Dermatitis exfoliative, AripiprazoleHyperprolactinaemia, Temozolomide-Diabetes insipidus. Computed monthly PRR with its $95 \%$ confidence interval of all test cases until July 2016 were used. CPA method was applied on the lower bound 95\% confidence interval of PRR (PRR-).

Results: With CPA-PRR method, the couples can be detected for Ustekinumab-Dermatitis exfoliative in June 2015, for AripiprazoleHyperprolactinaemia in April 2009 and for Temozolomide-Diabetes insipidus in and July 2012 These new safety issues were addressed by PRAC respectively in August 2013, August 2014 and April 2014. CPAPRR method allowed earlier signal detections of $2 / 3$ cases.

Conclusion: Our preliminary results on CPA-PRR method demonstrated its robustness in the early detection of pharmacovigilance signals. The proposed method could be applied in combination with disproportionality analysis in Pharmacovigilance signal detection after further investigations. Further sources of information/References

1. Evans SJ, Waller PC, Davis S. Use of proportional reporting ratios (PRRs) for signal generation from spontaneous adverse drug reaction reports. Pharmacoepidemiol Drug Saf 2001;10:483-6

2. Eudravigilance Expert Working Group. Guideline on the use of statistical signal detection methods in the Eudravigilance data analysis system (EMEA: 106464/2006 rev.1).

3. Kass-Hout TA, Xu Z, McMurray P, Park S, Buckeridge DL, Brownstein JS, et al. Application of change point analysis to daily influenza-like illness emergency department visits. J Am Med Inform Assoc 2012;19:1075-81

4. Xu Z, Kass-Hout T, Anderson-Smits C, Gray G. Signal detection using change point analysis in postmarket surveillance. Pharmacoepidemiol Drug Saf 2015;24:663-8

5. Trinh N, Sole E, Benkebil M. Benefits of combining change point analysis with Disproportionality Analysis in Pharmacovigilance signal detection. 33rd International Conference on Pharmacoepidemiology and Therapeutic Risk Management 
153 Local success is global success-addressing the challenges of implementing product risk minimization globally

\author{
J. Whitehead ${ }^{1}$, J.Kennedy $^{1}, \underline{\text { J. Ho }}{ }^{\mathbf{1}}$ \\ ${ }^{1}$ Navitas, Denmead, UK
}

Background: A key component of Pharmacovigilance [1] is understanding the risks of a product and implementing appropriate risk minimisation measures (RMMs) when needed. The concept is intuitive, but in practice, presents challenges.

One of the most significant challenges is the inconsistency of expectations from regulators. This is perhaps most clearly embodied in the different risk management documents (RMDs) that Health Authorities require. This challenge is compounded by emerging regulations in many countries requiring a risk management document.

Internally, companies face global implementation challenges because affiliates serve as the primary interface to local stakeholders. However, not every local office has the same resource model. There is variability in which roles are present, who they report into, and how much time they devote to PV-related activities.

Objective: The objective of this study is to identify a global safety risk management (SRM) operating model rooted in a consistent $\mathrm{B} / \mathrm{R}$ position while allowing sufficient flexibility to meet national requirements along with simplifying management and oversight.

Methods: Data was gathered via surveys of over 200 affiliates across 80 countries to identify common risks and challenges that present barriers to successful RMM implementation. In addition, lessons learned from MAHs and best practices from our industry forums were analyzed and synthesized into a model for success.

Results: Headquarter safety governance models have largely shifted to a single $\mathrm{B} / \mathrm{R}$ position with an associated "Core RM" document but have had mixed results with local RMD and RMM implementation using communication channels that predominately flow from HQ to affiliates. At the affiliate level, survey responses show that areas of higher risk exposure involve cross-functional activities like label updates; alignment of local documents and procedures to those in headquarters; and current information on local regulations. These all directly impact a company's ability to implement RMMs at a local level successfully.

Discussion/Conclusion: A successful SRM operating model begins with a region-agnostic, globally implementable SRM strategy for a product. This is set by the cross-functional product team in HQ but must be informed by knowledge and experience from cross-functional teams at the local level, because it is these teams who are best positioned to determine the feasibility of implementation and effectiveness measurement of any RMM in their markets. In addition, a global tracking mechanism enabling dashboards is needed to simplify oversight and achieve inspection readiness. The overall message is that global patient SRM is only achievable through local success in each market.

Further sources of information/References:

1. Pharmacovigilance Risk Assessment Committee. EMA/790863/2015. PRAC strategy on measuring the impact of Pharmacovigilance activities. European Medicines Agency; 2016
154 Rabies Vaccine-Induced Adverse Drug Reactions (ADRs): What Kind of Serious ADRs?

M. Damin-Pernik ${ }^{1}$, F. Bellet ${ }^{1}$, F. Moulis ${ }^{2}$, Al. Ruellan ${ }^{3}$, A. LilloLe-Louet ${ }^{4}$, P. Poujol ${ }^{5}$, T. Goulenok ${ }^{6}$, F. Lucht ${ }^{7}$, M.N. Beyens ${ }^{1}$

${ }^{1}$ Regional Pharmacovigilance Department, Saint-Etienne, France, ${ }^{2}$ Regional Pharmacovigilance Department, Toulouse, France, ${ }^{3}$ Regional Pharmacovigilance Department, Nantes, France, ${ }^{4}$ Regional Pharmacovigilance Department, Paris, France, ${ }^{5}$ Centre anti-rabique de l' Institut Pasteur, Paris, France, ${ }^{6}$ Internal Medicine Department, Paris, France, ${ }^{7}$ Department of Infectious Diseases, Saint-Etienne, France

Introduction: Rabies is a viral disease $100 \%$ fatal but which can be prevented with vaccines and immunoglobulins. Three types of inactivated cell culture vaccines have been licensed in France for pre- and post-exposure prophylaxis of rabies: the Pasteur and Merieux vaccines and Rabipur. They are considered safe and well tolerated. However, the literature reports various cases of ADRs like acute demyelinating encephalomyelitis, immune thrombocytopenia or peripheral neuropathy. The aim of this study was to characterize the profile of serious ADRs reported with the rabies vaccines in France.

Material and methods: A descriptive retrospective study was conducted from the FPVD. All cases of ADRs in which a rabies vaccine was considered as "suspect" between January 1st, 2010 and November 25th, 2015 were included. Serious ADRs (SADRs) were described. We checked if they were labelled in the summary of product characteristics.

Results: Seventy-five cases were reported. Mean age of subjects was 32.3 years old and sex ratio (F/M) was 1.6. Twenty-one cases $(28 \%)$ were coded as serious; for patients aged 37 with the same sex ratio. Among serious cases, 12 were unexpected, including 2 cases registered as macrophagic myofasciitis (entity not clinically recognized), 1 acute disseminated encephalomyelitis, 1 Parsonage-Turner, 1 peripheral neuropathy, 2 vestibular disorders, 1 thrombophlebitis, 2 vasculitis, 1 ageusia and 1 ulcero-necrotic gingivitis. Rabies vaccine was the only suspected drug in 8 cases (38\%). All these adverse effects have a favorable outcome except cases of macrophagic myofasciitis, ParsonageTurner, peripheral neuropathy and vestibular disorders with a follow-up of 6 months.

Discussion / Conclusion: We identified serious and unexpected ADRs but the vaccine was often associated with other suspected drugs (mainly other vaccines), outcome is not favorable for some neurological SADRs but it may be due to the short follow-up time. The benefit/risk of rabies vaccines appears positive considering the fatal issue of the disease.

\section{Evaluation of Compliance to the Timing of Childhood Vaccinations Following Introduction of the Meningococcal B Vaccine in the UK}

\author{
$\underline{\text { J. Wong }^{1}}$, K. Donegan ${ }^{1}$, S. Seabroke ${ }^{1}$, P. Bryan ${ }^{1}$ \\ ${ }^{1}$ Pharmacoepidemiology Research \& Intelligence Unit, Medicines \\ And Healthcare Products Regulatory Agency, London, UK
}


Background: In September 2015, the UK implemented a routine, national childhood immunisation programme with meningococcal B (MenB) vaccine with three doses at 8,16 , and 52 weeks of age. Local reactions and fever are known risks with Bexsero and before the start of the programme, it was identified that it was important to monitor for any potential impact the introduction of Bexsero had on compliance with subsequent doses of other routine vaccines.

Aim: To investigate any delays in receiving 2 nd and 3 rd doses of Bexsero and other routine childhood vaccinations to understand the acceptability of the known risks to parents.

Methods: Within the Clinical Practice Research Datalink, two study cohorts were identified based upon date of birth: a historical cohort born 01/07/2013-30/06/2015 who received the 1st dose of either the rotavirus or pneumococcal conjugate vaccine (PCV) aged 6-10 weeks and a postBexsero cohort born on or after 01/07/2015, and therefore eligible for Bexsero as part of the routine schedule, who received a 1st dose of Bexsero, rotavirus vaccine, or PCV aged 6-10 weeks. Birthdates were assumed to be the $15^{\text {th }}$ of the month. Comparisons were made across the two cohorts for the compliance with, and timing of, subsequent doses of rotavirus and pneumococcal vaccines using Kaplan-Meier curves and the log-rank test.

Results: 17,432 infants received $1^{\text {st }}$ dose of Bexsero at 6-10 weeks. By 26 weeks, $94.7 \%$ of those had received their 2nd dose and by 62 weeks $84.6 \%$ had received their 3 rd dose. For PCV, there were no differences in the age at $1 \mathrm{st}$ vaccination in the identified cohorts prior to and after introduction of the MenB vaccine programme $(\mathrm{P}=0.863)$. By 26 weeks, approximately $95 \%$ had received their 2 nd dose in both cohorts $(\mathrm{P}=0.1)$ and by 62 weeks, approximately $86 \%$ of infants had received their 3rd dose $(\mathrm{P}=0.068)$. For rotavirus, the age at vaccination with the 1 st dose showed no differences between cohorts $(\mathrm{P}=0.713)$. By 17 weeks, $94 \%$ of infants had received their 2 nd dose $(\mathrm{P}=0.843)$ in both cohorts. The times to 2 nd and 3rd doses of Bexsero were in line with other vaccines.

Conclusion: The incidence of adverse events following the $1^{\text {st }}$ dose of Bexsero does not appear to have had a negative effect on adherence to the schedule for subsequent doses of Bexsero or other vaccines. This suggests that the level of reactogencitiy seen with Bexsero is acceptable to parents given the potential benefits of the vaccine.

\section{Intussusception cases in an Italian Pediatric Population}

R. Da Cas ${ }^{1}$, F. Menniti-Ippolito ${ }^{1}$, R. Rossi ${ }^{2}$, U. Raucci ${ }^{2}$, $\overline{\text { A. Mechio }}^{3}$, D. Altavilla ${ }^{3}$, F.Trotta ${ }^{4}$, and the Multicenter Study Group on Drug and Vaccine Safety in Children

${ }^{1}$ Italian National Institute Of Health, Rome, Italy, ${ }^{2}$ Bambino Gesù Pediatric Hospital, Rome, Italy, ${ }^{3}$ Department of Biomedical and Dental Sciences and Morphofunctional Imaging, University of Messina, Messina, Italy, ${ }^{4}$ Department of Epidemiology, Lazio Regional Health Service, Rome, Italy

Background. Intussusception (IS) is the most common cause of acute intestinal obstruction in infants with a background of incidence differential by geographical age in Italy [1]; IS also represents an identified risk with rotavirus vaccinations (RV) [2]. RV has been recently included in the National Immunization Program (2017-2019) in Italy, thus being actively offered to all children population and fully reimbursed by the Italian NHS. To actively monitoring the safety of RV an active surveillance of IS events has been set up.

Objective: The aim of this pilot study was to describe IS cases in a pediatric population.
Methods: An active surveillance on drug and vaccine safety in children is ongoing in 11 clinical centers in Italy. All children hospitalized through the local Paediatric Emergency Department for selected acute clinical conditions of interest were enrolled in the study. Parents were interviewed on child's use of drugs and vaccines preceding the onset of symptoms that led to the hospitalization. Demographic information and related comorbidity were also available. Cases with a diagnosis of IS were retrieved from all admissions. Moreover, each IS case was characterized with the following information: type of IS (ileo-ileal/ileo-colic), recurrence, diagnostic criteria, microbiological analyses and IS outcome. Descriptive analysis was performed

Results: During the pilot phase from October 2015 to April 2017, 51 IS cases were collected with a mean age of 37 months and a prevalence of males $(69 \%)$. The majority were incident IS cases (88\%), while only 6 IS cases $(12 \%)$ were recurrences. In five cases microbiological examination was performed and 1 case was positive to rotavirus. Ultrasound scan was very frequent to confirm the diagnosis (44 out of 51 case), while enema and surgery were less performed (13 and 5 IS cases, respectively). In 14 out of 51 cases $(27 \%)$ a previous exposure to vaccines were reported, only in 1 case the RV was involved. The median time from vaccination to IS was 48 days (range 4-72).

Conclusion. The pilot study confirm that the surveillance system already set up is able to identify IS cases. Moreover, the information retrieved allow to evaluate cases using the Brighton Collaboration criteria for the case definition of IS. This surveillance represents a tool which is ready to estimate the association of IS with vaccinations in a pediatric population, allowing a continuous real time monitoring of the benefit/risk profile of RV.

\section{Further sources of information/References}

1. Trotta F, Da Cas R, Bella A, Santuccio C, Salmaso S. Intussusception hospitalizations incidence in the pediatric population in Italy: a nationwide cross-sectional study. Ital J Pediatr 2016 27;42:89

2. Rotavirus vaccines. WHO position paper-January2013. Wkly Epidemiol Rec 2013;88:49-64

\section{Recording and assessing medication errors within a spontaneous reporting system: first results}

U. Köberle ${ }^{1}$, T. Stammschulte ${ }^{1}$, L. Prause ${ }^{1}$, H. Pachl ${ }^{1}$, U. Gundert-Remy $^{1,2}$, K. Bräutigam ${ }^{1}$

${ }^{1}$ Drug Commission Of The German Medical Association, Berlin, Germany, ${ }^{2}$ Institute for Clinical Pharmacology and Toxicology, Charité, Berlin, Germany

Introduction: In a systematic review the prevalence of medication errors (MEs) with severe sequelae was low [1]. A German study, however, showed that $20 \%$ of adverse drug reactions (ADR) in outpatients leading to hospitalisation were avoidable MEs [2]. In conformity with the new EU pharmacovigilance legislation the Drug Commission of the German Medical Association (DCGMA) established a subsystem for evaluating MEs within the spontaneous reporting system [3].

Aims: To evaluate

- the feasibility of recording and assessing MEs using the existing structures of DCGMA;

- the suitability to deduce risk minimising intervention strategies from the obtained information. 
Methods: Healthcare professionals are invited to report MEs that led to (or may lead to) harm to patients. Case reports are assessed in analogy to other spontaneous reports of ADRs within the DCGMA. MEs are coded with MedDRA and forwarded to national and international institutions (ICH E2B format). Quantitative and qualitative analyses are performed. Results: From 4 January 2016 to 2 May 2017, 88 case reports (45 male, 43 female) of MEs were reported (among a total of over 5000 ADR reports). Of those 88 reports, 44 reports were considered as serious (including 3 fatal cases). Most frequently, application errors were reported $(n=27)$, followed by prescribing errors $(n=21)$, and dispensing errors $(n=14)$. Elderly patients ( $>65$ years, $n=52$ ) were the most affected group. Case reports of special interest were intensely discussed with experts and the German drug authorities. In several cases, the ME reports triggered risk-reducing measures. Conclusion: In general, the recording and the assessment of MEs within the existing structures of the spontaneous reporting system of the DCGMA is feasible. There were several case reports that triggered risk minimising measures. The assessment of MEs is an important aspect of the pharmacovigilance system. Therefore, physicians and other healthcare professionals should be encouraged to report MEs without blame or fear of punishment.

Further sources of information/References:

1. Ferrah N, et al. Systematic Review of the Prevalence of Medication Errors Resulting in Hospitalization and Death of Nursing Home Residents. J Am Geriatr Soc 2017; 65: 433-42

2. Rottenkolber $\mathrm{D}$, et al. Adverse drug reactions in Germany: direct costs of internal medicine hospitalizations. Pharmaoepidemiol Drug Saf 2011; 20: 626-34

3. Köberle $\mathrm{U}$, et al. Pilot project of recording and assessing medication errors within the German spontaneous reporting system. Poster at the 15th ISoP Annual Meeting in Prague, 27-30 October, 2015

\section{Non-Medical Prescriber Experiences of Training and Competence to Report Adverse Drug Reactions}

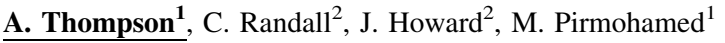 \\ ${ }^{1}$ University of Liverpool, Liverpool, UK, ${ }^{2}$ North West Medicines \\ Information Centre, Liverpool, UK
}

Background: UK Epidemiological data suggest that Adverse Drug Reactions (ADRs) are responsible for significant healthcare utilisation and account for $6.5 \%$ of hospital admissions. The Yellow Card Scheme (YCS) is the cornerstone of pharmacovigilance in the UK relying on spontaneous reports alongside data from clinical trials and pharmacovigilance studies. Non-medical prescribing was introduced to allow patients quicker access to the medicines without compromising patient safety. Healthcare professionals have a responsibility to report to the YCS but Non-Medical Prescribers (NMPs) are often better placed than non-prescribers to identify ADRs. It is therefore important that NMPs receive appropriate initial and subsequent training on the identification and reporting of ADRs.

Aim: To gain an understanding of NMP confidence in identifying and reporting ADRs, explore NMP prescribing habits and engagement with the YCS and investigate the desire of NMPs for future training in the identification and reporting of ADRs.

Methods: An online questionnaire was developed by members of Liverpool Health Partners Yellow Card Working Group and Yellow Card Centre North West to address these aims. The questionnaire was circulated via local and regional NMP Leads in the North West of England. The flow of the questionnaire was dependent upon the responses provided.
Results: 570 responses were available for analysis. The majority of responders were nurses $(68.1 \%)$ or community practitioners $(21.1 \%)$. Less than half $(\mathrm{n}=219 ; 38.4 \%)$ reported submitting a Yellow Card to the YCS, and the majority of those individuals have submitted five or less Yellow Cards; 28 responders reported more than five submissions. Being professionally qualified for more years $(B=-0.30, \mathrm{p}<0.0005 ; 95 \% \mathrm{CI}$ 0.96 to 0.99 ), and receiving additional training support about the YCS $\left(X^{2}=14.7, \mathrm{p}<0.0005\right)$ were associated with an increased likelihood of submitting to the YCS. There was a positive linear relationship between confidence in identifying ADRs and likelihood of reporting to YCS. There was also a significant association between confidence and the number of Yellow Cards submitted ( $<$ 0.0005), but this did not follow an absolute linear trend. The most common reason given $(n=261)$ for never having reported to the YCS was "I have never seen an adverse drug reaction". Conclusion: Training appears to give NMPs confidence in reporting ADRs, but there seems to be a gap in actually identifying ADRs given the comment that most had never seen an ADR. Strategies for improving the translation of theoretical knowledge about ADRs into practical skills in identifying ADRs, and subsequently reporting them, will be important.

\section{Does a Good Institutional Reporting Culture Extend to the Reporting of Yellow Cards}

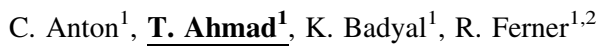 \\ ${ }^{1}$ West Midlands Centre for Adverse Drug Reaction Reporting, City \\ Hospital, Birmingham, UK, ${ }^{2}$ Institute of Clinical and Experimental \\ Medicine, University of Birmingham, Birmingham, UK
}

Introduction: A safe culture is one in which leaders and frontline staff take a shared responsibility to ensure care is delivered safely. Part of a safety culture is that staff have a positive perception of psychological safety, teamwork, and leadership, and feel comfortable discussing errors [1].

Aim: The purpose of this study was to examine whether acute hospitals that are good at reporting patient safety incidents were equally good at reporting adverse drug reactions (ADR).

Methods: We gathered data on patient safety incidents regarding all acute hospitals in the West Midlands region reported to the NHS National Reporting and Learning System (NRLS) from $1^{\text {st }}$ April 2013-31 $1^{\text {st }}$ March 2016. We used overnight bed availability and occupancy data from NHS England to calculate bed days, and expressed rates per million bed days. Rates were compared using Spearman correlation coefficient.

Results: We identified 17 West Midland acute hospital organisations, of which five submitted 36 months' data. The hospitals with the highest incident rates are shown in the table.

Table: Correlations between hospital reporting of incidents and ADRs
\begin{tabular}{|l|l|l|l|}
\hline Year & $\begin{array}{l}\text { Hospital with } \\
\text { complete datasets }\end{array}$ & Spearman rho & P \\
\hline $2013 / 14$ & 13 & 0.35 & 0.91 \\
\hline $2014 / 15$ & 12 & 0.32 & 0.89 \\
\hline $2015 / 16$ & 11 & 0.25 & 0.85 \\
\hline
\end{tabular}

The incident rates ranged from 3726-21,784 per million bed days. The ADR reporting rate ranged from $0-82$ per million bed days.

We considered whether hospitals that sent complete datasets might have better reporting systems, but over the three years the five hospitals with complete incident data sent on average 17.6 ADRs per million bed days compared with 29.8 ADRs per million bed days from the incomplete dataset group of hospitals. 
Conclusions: Reporting patient safety incidents and reporting ADRs do not correlate. There are a number of variable external factors that may affect patient safety reporting. We found no evidence that hospitals that submitted complete datasets were better at reporting ADRs.

Discussion: The lack of correlation may be because reporting systems may differ, different members of staff may be responsible for reporting different incidents, or organisational culture may affect reporting incidents and ADRs differently. We have looked at ADRs with all incidents reported and not only medicine-related incidents reports and there may be differences in how staff consider the two types of incidents. Unifying the reporting systems could improve ADR reporting, and could be considered. Further sources of information/References

1. The Health Foundation. Safety culture: What is it and how do we measure it?. Available from: http://www.health.org.uk/sites/ health/files/SafetyCultureWhatIsItAndHowDoWeMeasureIt.pdf [Accessed 25 April 2017]

\section{Safety of meningococcal group B vaccine: experience from the $\mathrm{UK}$}

\author{
S. Seabroke ${ }^{1}$, P. Bryan ${ }^{1}$ \\ ${ }^{1}$ Medicines \& Healthcare products Regulatory Agency, London, UK
}

Introduction: In September 2015, the UK became the first country in the world to introduce a routine meningococcal group B vaccine (Bexsero) infant immunisation programme. The vaccine is offered at 2, 4 and 12 months of age alongside other routine vaccinations. Based on clinical trial data, Bexsero is known to be more reactogenic than other routinely used vaccines with fever as the main systemic adverse event. Due to the high rate of fever with this vaccine, febrile convulsions are a potential risk. The MHRA proactively monitors the safety of any new vaccine using spontaneous data and electronic healthcare record data from the Clinical Practice Research Datalink (CPRD)

Aim: To monitor the rate of convulsions post-vaccination with Bexsero and determine if the rate had increased compared with post-vaccination rates prior to the introduction of Bexsero.

Methods: In addition to individual case review, statistical 'observed vs expected' (O/E) analyses were conducted for convulsions using CPRD data. Historical post-vaccination (within 7 days) incidence rates were calculated to determine a background incidence rate and together with Bexsero exposure estimates an 'expected' number of events was derived. The 'observed' number of spontaneous reports received was then compared to the 'expected'. An ecological analysis to compare post-vaccination convulsion incidence rates in the time period before ( 5 years) and time period after the introduction of Bexsero was also conducted using CPRD data. Temporal trends were performed using Poisson regression analysis.

Results: Between September 2015-October 2016, approximately 2 million doses of Bexsero were administered to at least 1 million children across the UK. A total of 1055 spontaneous reports were received including 372 reports for fever and 40 for all convulsions. The convulsion reports were well within the expected range in the 7 days following vaccination (age-adjusted $\mathrm{O} / \mathrm{E}$ ratio: 0.14 [95\% CI 0.1-0.2]). The ecological analysis also did not show an increased rate of convulsions post-vaccination with the addition of Bexsero to the routine schedule: at 2 months of age, incidence rate ratio (IRR) 1.10 [95\% CI 0.38-3.15]; at 4 months of age, IRR 1.72 [95\% CI 0.49-6.09], at 12 months of age, IRR 0.47 [95\% CI 0.12-1.90].

Conclusions: The few reports of convulsions reported following vaccination with Bexsero are well within the expected rate following routine vaccination with other vaccines. There is no evidence currently of an increased risk of convulsions following Bexsero however this will remain under close review by the MHRA.

\section{Idiosyncratic Drug-Induced Liver Injury: A 20- Year Update of the Cases Enrolled by the Spanish dili Registry}

M. Slim ${ }^{1}$, J. Sanabria ${ }^{1}$, M. Robles-Diaz ${ }^{1}$, I. Medina-Caliz ${ }^{1}$, R. Sanjuán-Jiménez ${ }^{1}$, A. González-Jiménez ${ }^{1}$, A. Ortega ${ }^{1}$, M. García-Cortés ${ }^{1}$, B. Garcia-Muñoz ${ }^{1}$, M.C. Fernandez ${ }^{2}$, A. Castiella ${ }^{3}$, E. Zapata ${ }^{3}$, H. Hallal ${ }^{4}$, I. Conde ${ }^{5}$, M. Prieto ${ }^{5}$, E. Montane ${ }^{6}$, R.M. Morillas ${ }^{6}$, G. Soriano ${ }^{7}$, E. Roman ${ }^{7}$, J.M. Navarro ${ }^{8}$, M. Jimenez ${ }^{9}$, A. Aldea ${ }^{10}$, M. Hernández-Guerra ${ }^{10}$, P. Gines $^{11}$, S. Blanco ${ }^{12}$, J.M. Moreno ${ }^{13}$, M. Romero-Gomez, E. Gomez-Dominguez ${ }^{15}$, R. Andrade ${ }^{1}$, M.I. Lucena ${ }^{1}$

${ }^{1}$ Universidad de Malaga, Malaga, Spain, ${ }^{2}$ Hospital Torrecardenas, Almería, Spain, ${ }^{3}$ Hospital de Mendaro, Mendaro, Spain, ${ }^{4}$ Hospital Morales Meseguer, Murcia, Spain, ${ }^{5}$ Hospital Universitario y Politécnico La Fe, València, Spain, ${ }^{6}$ Hospital Germans Trias i Pujol, Barcelona, Spain, ${ }^{7}$ Hospital de la Santa Creu i Sant Pau, Barcelona, Spain, ${ }^{8}$ Agencia Sanitaria Costa del Sol, Marbella, Spain, ${ }^{9}$ Hospital Regional de Málaga, Málaga, Spain, ${ }^{10}$ Hospital Universitario de Canarias, Santa Cruz de Tenerife, Spain, ${ }^{11}$ Hospital Clinic de Barcelona,Barcelona, Spain, ${ }^{12}$ Hospital Universitario Basurto, Bilbao, Spain, ${ }^{13}$ Complejo Hospitalario de Albacete, Albacete, Spain, ${ }^{14}$ Hospitales Universitarios Virgen Macarena-Virgen del Rocío, Seville, Spain, ${ }^{15}$ Hospital 12 de Octubre,Madrid, Spain

Background: Idiosyncratic drug induced liver injury (DILI) is a rare adverse drug reaction that poses major challenges to healthcare practitioners and regulatory agencies.

Objective: We aimed to update the clinical characteristics and outcomes of DILI patients, and the drugs frequently implicated in hepatotoxicity in Spain.

Methods: We analyzed 915 DILI cases (842 single episodes, 55 rechallenges, 18 double-episodes due to different drugs) in 857 patients included in the Spanish DILI Registry from 1994 to 2015. Cases were adjudicated using expert clinical judgment/RUCAM scale and compared according to pattern of liver damage (hepatocellular, HC; cholestatic, Chol or mixed, Mix).

Results: The cohort median age was 57 years (range: 11-90 y) with a mean body mass index of $25.8 \pm 3.8 \mathrm{~kg} / \mathrm{m}^{2}$. Male gender predominated $(52 \%)$. HC, Chol and Mix patterns of liver damage were identified in $65 \%$, $18 \%$ and $17 \%$ of cases, respectively. More than half of the cases were of moderate severity (58\%). Patients with Chol and Mix pattern were older (median $64 \mathrm{y}$ and $62 \mathrm{y}$, respectively) than HC patients (median $52 \mathrm{y}$ ), $\mathrm{p}<0.001$. Anti-infectives, central nervous system, cardiovascular and anti-inflammatory agents were the most commonly implicated therapeutic classes accounting for 37, 14, 11 and 9\% of cases, respectively. Amoxicillin/clavulanate remains the agent responsible for the highest number of DILI ( $21 \%$ of cases). Substantial increase in anabolic androgenic steroidinduced hepatotoxicity was observed in recent years. A cluster of DILI cases reported to the registry (i.e. ebrotidine, tetrabamate, nimesulide, amoxicillin-clavulanate, Exolise ${ }^{\circledR}$, Epistane ${ }^{\circledR}$ ) contributed to adoption of regulatory measures.

Conclusions: The pioneering prospective Spanish DILI Registry proved to be very valuable for in-depth clinical phenotyping of hepatotoxicity, providing consistent figures in clinical characteristic outcomes and 
implicated drugs. It also constitutes an important tool for public health promotion in postmarketing drug surveillance.

Funding: Research grants from AEMPS and FEDER (PI15/01440, PI 16/01748). CIBERehd by ISCIII.

\section{Analysing Drug-Host Interplay in Clinical Presentation of Drug-Induced Liver Injury using Data Mining Techniques}

A. Gonzalez-Jimenez ${ }^{1}$, K. McEuen ${ }^{2}$, M. Robles-Diaz ${ }^{1}$, I. MedinaCaliz $^{1}$, M. Slim ${ }^{1}$, J. Sanabria ${ }^{1}$, A. Cueto-Sanchez ${ }^{1}$, M.Chen ${ }^{2}$, A. Suzuki ${ }^{3}$, M. Isabel Lucena ${ }^{1}$, C. Stephens ${ }^{1}$, R.J. Andrade ${ }^{1}$

${ }^{1}$ Ibima, H. Virgen De La Victoria, Universidad De Malaga, Ciberehd, Malaga, Spain, ${ }^{2}$ Division of Bioinformatics and Biostatistics, National Center for Toxicological Research, Jefferson, USA

${ }^{3}$ University of Duke, Durham, USA

Background Drug-induced liver injury (DILI) can present with diverse clinical phenotypes even when the same agent is involved, while some drugs cause signature injury patterns (e.g., acute hepatocellular injury by acetaminophen; acute cholestasis by anabolic steroids). The diversity in clinical phenotypes associated with most of the causal drugs may be partly explained by multi-phasic interplays between drug properties and host

Aims We aimed to explore and characterize drug-host interplay in initial biochemical presentations in a large well-characterized Spanish DILI patient population.

Methods We analyzed 695 cases (probable or higher in the RUCAM causality assessment) enrolled in the Spanish DILI registry. Clinical information on demography, causal drugs, comorbidities, co-medications, and clinical manifestations including initial biochemical presentations, was collected at the time of study enrollment. Information on drug properties of the causal drugs was obtained from the literature as well as the Liver Toxicity Knowledge Base (CPT 2013; 93:409). Cases manifested as mixed biochemical injury pattern were excluded from this analysis. Logistic regression model (hepatocellular vs. cholesteric injury) was used to assess each host/drug factor pair along with their interaction term. Once a statistically significant interaction term was identified, separate logistic regression models were applied to determine whether a divergent relationship between liver injury and drug property exists among host factor subgroups.

Results A total of 68 host factors and 71 drug properties were analyzed. Among 4828 host/drug factor pairs assessed, 26 pairs were identified as statistically significant interactions. The identified pairs included but were not limited to age-hepatic metabolism, hyperlipidemia-reactive metabolite, and allergic records-drug electronegativity. Further subgroup analyses revealed that older age ( $>60$ years) was associated with cholesteric injury only for drugs which are not significantly metabolized in the liver (OR $[95 \% \mathrm{CI}]=3.9$ [2.0-7.7], $p=0.0001)$, such as amoxicillin-clavulanate, nitrofurantoin or methotrexate, but not for drugs with significant hepatic metabolism $(\mathrm{OR}[95 \% \mathrm{CI}]=1.6[0.9-2.9], p=0.09)$, such as isoniazid or flutamide.

Conclusion Data-mining analysis using logistic regression models with an interaction term identified multiple potential combinations of host/drug factors, which may interact and modify initial biochemical presentations in DILI. The identified interactions may aid in better understanding the heterogeneity of clinical presentations in DILI cases. Further methodological implementation to discover latent associations among multiple factors ( $>2$ factors) are warranted.

Funding: AEMPS, FEDER-PI15/01440, CIBERehd-ISCIII
164 Near Real-Time Monitoring of the Anticipated Benefit-Risk of Vaccines Using an Interactive Dashboard: a Proposition from ADVANCE

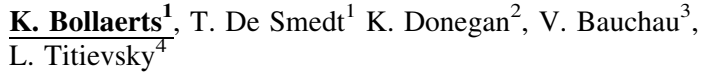

${ }^{1}$ P95, Kessel-lo, Belgium, ${ }^{2}$ Medicines \& Healthcare products Regulatory Agency, London, UK, ${ }^{3}$ GSK, Wavre, Belgium, ${ }^{4}$ Pfizer, New York, USA

Background: New vaccines are launched based on their Benefit-Risk (B/ R) profile anticipated from the clinical development. Proactive postmarketing surveillance is necessary to assess whether the vaccine meets its expectations and to support public health and regulatory decision making. There are several, typically not integrated, facets of post-marketing vaccine surveillance; the surveillance of vaccination coverage, vaccine safety, effectiveness and impact. Although formal benefit-risk (BR) assessments, by which the benefits of a medicine are offset against its risks at one point in time, are increasingly performed, near real-time (integrated) monitoring of coverage, risks and benefits is not yet routinely implemented in practice and in particular, for vaccines.

Aim: To assess the feasibility and added value of using an interactive dashboard as a potential methodology for near real-time BR monitoring of vaccines, demonstrated using simulated electronic healthcare record data.

Methods: For developmental purposes, we simulated data mimicking the introduction of rotavirus vaccination (RVV) in the UK. We built a webapplication with an interactive dashboard for BR monitoring of pre-specified health benefits and risks. The dashboard allows end-users to select certain parameters including expected vaccine effectiveness, age groups, time periods, to calculate the incremental net health benefit (INHB) and incremental benefit risk ratio (IBRR) for different sets of preference weights and to conduct sensitivity analyses. The individual components of BR (vaccination coverage, benefits and risks) were also monitored. Lastly, we assessed the dashboard's added value by user testing amongst a group of stakeholders experienced in the post-marketing monitoring of vaccines.

Results: We demonstrated the dashboard using simulated data on the BR of RVV introduction, accounting for excess risk of intussusception and reduced incidence of rotavirus gastroenteritis. From the user-testing it was concluded that the dashboard is a useful tool for visualizing individual BR components, for examining the impact of user selected parameters on the outcomes, and could allow for transparent, consistent and communicable benefit-risk monitoring. However, the real world applicability/utility of the BR dashboard strongly depends on the timely availability of, and access to, data on vaccination coverage, benefits and risks. Further work is needed to identify the optimal scenario(s) when such dashboard and monitoring would be most useful.

Conclusions: The proposed benefit-risk dashboard is promising. Availability of near real-time data remains a concern and will be tested in a further work with actual databases.

\section{Safety profile of direct-acting antivirals for hepatitis c: an analysis of italian spontaneous reporting system database}

\author{
P. Maria Cutroneo ${ }^{1}$, C. Ferrajolo ${ }^{2}$, E. Marotta ${ }^{3}$, N. Pellegrino ${ }^{1}$,

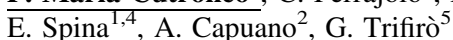 \\ ${ }^{1}$ Sicilian Regional Pharmacovigilance Centre, Messina, Italy, \\ ${ }^{2}$ Department of Experimental Medicine, Campania Regional Center
}


of Pharmacovigilance and Pharmacoepidemiology, University of Campania, Naples, Italy, ${ }^{3}$ Italian Medicines Agency, Pharmacovigilance Office, Rome, Italy, ${ }^{4}$ Dept. of Clinical and Experimental Medicine, University of Messina, Messina, Italy, ${ }^{5}$ Dept. of Biomedical and Dental Sciences and Morpho-functional Imaging, University of Messina, Messina, Italy

Background: Treatment of chronic hepatitis $\mathrm{C}$ virus (HCV) infection has dramatically improved since the advent of direct anti-viral agents (DAAs), including daclatasvir, dasabuvir, sofosbuvir, ledipasvir /sofosbuvir, simeprevir and ombitasvir / paritaprevir / ritonavir. Although clinical trials have demonstrated these drugs to be well tolerated, recent post-marketing data raise concerns about risk of hepatitis B re-activation, induction or recurrence of hepatocellular carcinoma $(\mathrm{HCC})$ and severe bradycardia in association with amiodarone.

Aim: To provide an overview of DAAs safety using the Italian spontaneous reporting system (SRS) data.

Methods: We identified all suspected adverse drug reactions (ADRs) concerning DAAs during the period January 2014-April 2017 in Italian SRS. We described the frequency of DAA class- and compound-specific ADRs by system organ class (SOC) and Preferred Term (PT). As measure of disproportionality, Reporting Odds Ratio with 95\% Confidence Interval at level of Standardized MedDRA Queries (SMQs) and PT terms was calculated.

Results: Out of 160,956 ADR reports collected in Italian SRS, 1940 (1.2 $\%)$ concerned DAAs. Sofosbuvir was indicated as one of suspected DAA in 881 (45.4\%) ADR reports, ledipasvir/sofosbuvir in 466 (24.0\%), ombitasvir / paritaprevir / ritonavir in 452 (23.3\%), dasabuvir in 421 (21.7\%), simeprevir in $296(15.2 \%)$ and daclatasvir in 281 (14.5\%) cases. The mean age $( \pm \mathrm{SD})$ of DAA users was $62.9( \pm 11.3)$ years. The spontaneous reports regarded most commonly males $(\mathrm{n}=1089 ; 56.1 \%)$ than females $(\mathrm{n}=825 ; 42.5 \%)$.

Serious ADRs, including 66 fatal cases, accounted for $45.7 \%(n=886)$ of DAA-related reports.

Reported ADRs were mainly general and administration site disorders $(\mathrm{n}=451 ; 23.2 \%)$, dermatological adverse reactions $(\mathrm{n}=349 ; 18.0 \%)$ and hematological disorders $(n=323 ; 16.6 \%)$. In particular, the most represented adverse effects were anaemia $(\mathrm{n}=287)$, asthenia $(189)$, pruritus (122), hyperbilirubinaemia (90), insomnia (88), headache (84), fatigue (80). Out of the total 1940 reports, 33 concerned drug ineffectiveness and 12 about disease progression. Only 3 cases of hepatitis B reactivation were retrieved in Italian SRS. Analyzing SMQs, DAAs resulted disproportionally associated with some serious ADRs, including acute renal failure, neoplasms, ischaemic heart disease, cardiac arrhythmias, and hepatic failure and other liver damage-related conditions.

Conclusions: Spontaneous reporting databases represent a useful source to evaluate the safety profile of marketed drugs. Our analysis shows several potential safety signals in association with DAAs, even though confounding factors related to underlying disease make it difficult to interpret these results. Further studies are needed to evaluate these risks, especially cardiac disorders, renal failure and hepatocellular carcinoma.

\section{Challenges and Resolutions in Integrating Electronic Adverse Drug Reaction (ADR) Reporting into UK Clinical Systems: A Case Study}

\author{
R. Owen ${ }^{1}$, S. Britton ${ }^{1}$, S. Vaughan ${ }^{1}$ \\ ${ }^{1}$ Vigilance, Intelligence and Research Group, Vigilance \& Risk \\ Management of Medicines Division, Medicines \& Healthcare \\ products Regulatory Agency, London, UK
}

Introduction: Integration of electronic Yellow Card reporting into clinical systems is a huge step forward in accessibility of the Yellow Card Scheme to healthcare professionals. It makes reporting of suspected ADRs easier to complete as much of the information needed can be automatically populated from patient records. An information standard for electronic Yellow Card reporting was developed in line with NHS GP Systems of Choice in England in 2012 [1]. In 2016, the MHRA integrated Yellow Card functionality within a second clinical system; VISION. Currently $34 \%$ of reports the MHRA receive are from clinical systems and volumes are continuing to increase.

Aim: To review the process for integration of Yellow Card reporting within a primary care clinical system and identify challenges experienced and how these were resolved.

Method: Coding practices were reviewed between the Vision clinical system and MHRA; this included terminologies used for describing medicinal products and clinical vocabulary. We also conducted a review of the issues identified during testing and submitted Yellow Cards to date. Results: It was identified that drug information from the NHS $\mathrm{dm}+\mathrm{d}$ dictionary were not compatible with the MHRA drugs dictionary and every Yellow Card submitted would require manual population of the drug term. A mapping of over 135,000 medicinal terms from the $\mathrm{dm}+\mathrm{d}$ dictionary to the MHRA's Drugs Dictionary was created to enable automatic processing of these reports and standardise coding practices. A mapping tool has also been developed so that any new terms in $d m+d$ can be continually mapped to the MHRA dictionary. The mapping allows MHRA to receive more accurate drug information from the patient record. During the testing phase, other challenges addressed included how to accurately transfer the recording of concomitant medication and medical history. In the first 9 months 257 reports from VISION have been submitted for a wide variety of medicinal products all to a high standard of quality.

Conclusion: Reporting directly from clinical systems improves access to Yellow Card reporting which can lead to quicker identification of possible drug safety issues. $45 \%$ of GP practices in the UK can now report through their clinical system. Testing with initial suppliers has highlighted challenges which have helped shape future integrations with clinical systems. The $\mathrm{dm}+\mathrm{d}$ mapping and the creation of the mapping tool has allowed reports to be accurately classified without requiring human intervention and improved consistency for medicinal product coding. This enables faster and more accurate reporting of ADRs.

Further sources of information/References:

1. NHS information Standard ISB 1582 Electronic Yellow Card Accessed on 12 January 2017 at www.isb.nhs.uk/library/ standard/243 and www.isb.nhs.uk/documents/isb-1582

\section{Comparison of the Performances of Data-Mining Methods for Signals Detection on two Spontaneous Reporting Databases}

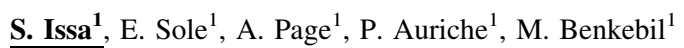

${ }^{1}$ French Medicines And Health Products Agency Surveillance Division, Adverse Events and Incidents Department, Paris, France

Introduction: Automated signal detection methods had become nowadays complementary tools in pharmacovigilance due to their ability to screen large spontaneous reporting databases and to identify in an untimely manner unknown drug- adverse reaction associations and new aspects of known drug- adverse reaction associations. Different signal detection algorithms are currently available and are implemented in routine 
pharmacovigilance processes. For instance, the French medicines agency uses a variant of the Bayesian method Gamma Poisson Shrinker (GPS) [1] for signal detection based on the French Pharmacovigilance Database. The European Medicines Agency applies a frequentist method, the Reporting Odds Ratio (ROR) [2] on the European pharmacovigilance database. The performances of these methods in generating signals of disproportionate reporting is likely to be dependent on the signal detection algorithms itself, the choice of the signaling criteria and the intrinsic characteristics of the reporting database [3].

Aim: To study the performances of data-mining techniques on the French pharmacovigilance database and European pharmacovigilance database.

Methods: Firstly, we measure the performance of data-mining on the French National Database and European pharmacovigilance database in their routine settings i.e using respectively the GPS method and ROR. Secondly, we use the same signal detection algorithm (ROR) on the two databases, in order to have a comprehensive understanding of the source of disparity of signal detection performances. The indicators used to assess the performance of data-mining are: the sensitivity, the specificity, the positive predictive value and the area under the curve. In order to measure these indicators, we made a reference dataset based on two known validated reference sets built by two working groups, the OMOP [4] and IMI PROTECT [5].

Results: Both analysis show differences in the performances of the signal detection on the French Pharmacovigilance database and European pharmacovigilance database. The sensitivity is the most sensible indicator.

Conclusion: The study demonstrates that the performances of data-mining methods for signal detection is dependent on the intrinsic characteristics of the adverse events reporting system database. These findings needs further investigations to determine whether there are other factors influencing the statistical power of spontaneous reporting databases to detect safety signals.

Further sources of information/References:

1. Ahmed I, Haramburu F, Fourrier-Réglat A, Thiessard F, Kreft-Jais C, Miremont-Salamé $\mathrm{G}$, et al. Bayesian pharmacovigilance signal detection methods revisited in a multiple comparison setting. Stat Med 2009;28:1774-92

2. van Puijenbroek EP, Bate A, Leufkens HGM, Lindquist M, Orre R, Egberts ACG. A comparison of measures of disproportionality for signal detection in spontaneous reporting systems for adverse drug reactions. Pharmacoepidemiol Drug Saf 2002;11:3-10

3. Candore G, Juhlin K, Manlik K, Thakrar B, Quarcoo N, Seabroke S, et al. Comparison of Statistical Signal Detection Methods Within and Across Spontaneous Reporting Databases. Drug Saf 2015;38:577-87

4. Ryan PB, Schuemie MJ, Welebob E, Duke J, Valentine S, Hartzema AG. Defining a Reference Set to Support Methodological Research in Drug Safety. Drug Saf 2013;36(S1):33-47

5. Wisniewski AFZ, Bate A, Bousquet C, Brueckner A, Candore G, Juhlin K, et al. Good Signal Detection Practices: Evidence from IMI PROTECT. Drug Saf 2016;39:469-90

174 Drug rash with eosinophilia and systemic symptoms (DRESS) syndrome induced by imatinib

F. Zgolli ${ }^{1}$, A. Zaiem ${ }^{1}$, F. Ben Salem ${ }^{1}$, I. Aouinti ${ }^{1}$, O. Charfi ${ }^{1}$, R. Daghfous ${ }^{1}$, E. Aidli Sihem ${ }^{1}$, K. Sarrah ${ }^{1}$

${ }^{1}$ Tunisian National Centre Of Pharmacovigilance, Tunis, Tunisia
Introduction: Imatinib is a selective BCR-ABL1 tyrosine kinase inhibitor. It is the treatment of choice in patients with locally advanced or metastatic gastrointestinal stromal tumours (GIST). It is considered as a safe drug, with mostly mild and reversible side effects. Most common side effects of imatinib include myelosuppression, nausea, vomiting, diarrhea, and skin rashes. DRESS syndrome is rarely reported with imatinib. Only few cases have been notified so far. We report a case of DRESS syndrome induced by imatinib.

Case report: A 46-year-old woman with past medical history of GIST developed two weeks after she had started on imatinib therapy (400 milligram per day) a pruritic skin rash limited to extremities. Skin examination showed an erythematous maculopapular eruption without fever or lymphadenopathy. The rest of the physical examination was normal. Laboratory findings showed a hepatic impairment and an elevated eosinophil count (3580 elements $/ \mathrm{mm}^{3}$ ). Plasma level of imatinib was 277 $\mathrm{ng} / \mathrm{ml}$ (within the therapeutic range). Imatinib was immediately discontinued. A favourable outcome was slowly observed after the drug withdrawal and under symptomatic treatment (oral prednisolone). Concomitant serology testing for HHV-6 revealed IgG antibodies, thus indicating a viral reactivation. The patient was firmly instructed to avoid imatinib in the future. Patch test was made, six weeks following the disappearance of the skin eruption, with imatinib diluted at $10 \%$ in Vaseline but was inconclusive.

Discussion/conclusion: The responsibility of imatinib was retained because of the suggestive chronology and the favourable outcome after the medication withdrawal. This case was evaluated likely (I3) according to Begaud and al. method of imputability [1] and was scored three (possible) according the RegiSCAR [2]. In literature, only 3 cases of DRESS syndrome associated to this drug were reported. The delay of onset varied from one week to three months. The main organs involved in these cases were live and skin. The outcome was favourable after drug withdrawal in a delay from to a month to three months. In conclusion, clinicians should be aware of the possibility of the occurrence of DRESS syndrome when prescribing imatinib.

Further sources of information/References :

1. Bégaud B, Evereux JC, Jouglard JB, et al. Unexpected or toxic drug reaction assessment. Actualisation of the method used in France. Therapie 1985;40:111-8

2. Descamps V, Ranger-Rogez S. Joint Bone Spine. 2014;81:15-21

\section{Adverse drug reactions reports for trastuzumab: an analysis of Brazilian pharmacovigilance database and VigiBase ${ }^{\circledR} /$ WHO and EudraVigilance/EMA}

\author{
F. Barcelos ${ }^{1,2}$, G. Matos ${ }^{1}$, E. Costa Lima-Dellamora ${ }^{1}$ \\ ${ }^{1}$ Faculty of Pharmacy- Federal University of Rio de Janeiro, Rio de \\ Janeiro, Brazil, ${ }^{2}$ National Cancer Institute, Rio de Janeiro, Brazil
}

Introduction: Trastuzumab is a monoclonal antibody (mAb) against human epidermal growth factor receptor 2 (HER2). Approved by FDA in 1998 , its toxicity profile had been complemented by adverse drug reactions (ADRs) detected during post-marketing, highlighting the importance of $\mathrm{mAb}$ 's surveillance. Pharmacovigilance databases play a key role effectively managing drug safety information coming from worldwide notifier centres [1-3]. In Brazil, pharmacovigilance activities are coordinated by the National Health Surveillance Agency (ANVISA). 
Aim: To analyse the suspected ADRs associated with trastuzumabe, reported to Brazilian National System (Notivisa) in contrast to VigiBase ${ }^{\circledR}$ and EudraVigilance.

Methods: From Notivisa, we performed a retrospective and exploratory study from 2008 to 2013 and analyzed patient's age and gender, as well as suspected ADR reaction groups/reporter group. The seriousness was also rated, according to ICH E2A guideline. The access to data was enabled through collaboration agreement between the University of Rio de Janeiro and the ANVISA. To comparison to other countries, the international databases VigiBase ${ }^{\circledR}$ and MedVigilance were accessed through their online platforms (www.vigiaccess.org and http://www.adrreports.eu).

Results: During the study period, we found 117 reports of trastuzumab (average 19.5 reports/year), all among 110 female patients (mean age of $51.2 \pm 12.7)$. In total, 235 suspected ADRs were recorded, with prevalence of those referred as General Disorders and Administration Site Conditions $(45.5 \%, \mathrm{n}=107)$, followed by Vascular Disorders $(12.3 \%, \mathrm{n}=29)$. Shivering/chills (35.3\%), hypo/hypertension (6.4\%), chest discomfort/pain (4.7\%) and flushing/hot flush $(3.8 \%)$ were the most frequently reported ADRs. Nevertheless, only $17.4 \%$ were considered serious, with particular focus on tachycardia $(n=9)$, febrile neutropenia $(n=3)$, hypersensibility reaction $(n=3)$ and severe infusion reactions, similar to shock $(n=4)$. There was no record of cardiac failure and only one of "cardiotoxicity". After confronting the findings with VigiBase ${ }^{\circledR}$ and EudraVigilance's reports, we observed that most were from women between 18 and 64 years (Notivisa $=77.8 \%$; VigiBase $=64 \%$; EudraVigilance $=51.5 \%$ ), with a different pattern of suspected ADR (Table 1).

Conclusion: Despite the shortage of suspected ADR for trastuzumab in Notivisa, most events refleted infusion-related reactions while too little was found referring to delayed ADRs. Its pattern of suspected ADR actually differed from those of VigiBase's and EudraVigilance's, which were very much similar to literature. However, cardiotoxicity had been previouslly reported on a cohort carried out in south of Brazil [4]. Our study indicates that pharmacovigilance actions in Brazil must be reoriented trough active monitoring, especially considering targeted therapy drugs, in order to reduce underreporting and improve safety and quality of cancer care.

\section{Further sources of information/References}

1. Clifford A, Hudis MD. Trastuzumab-Mechanism of Action and Use inClinical Practice. N Engl J Med 2007; 357:39-51

2. Albini A, Donatelli F, Noonan D, Delios, MM, Prisco D. Bringing new players into the field: onco-pharmacovigilance in the era of cardio-oncology. Intern Emerg Med 2012; 7: 99-101

3. Lindquist M. Vigibase, the WHO Global ICSR Database System: Basic Facts. Drug Information Journal 2008; 42:409-19

4. Lago LRG. Thesis of Master Degree. Rio Grande do Sul Federal University, 2015

Table 1 - Analytical overview of suspected ADRs for trastuzumabe, collected from Notivisa, VigiBase and EudraVigilance.

\begin{tabular}{|l|l|l|l|}
\hline Reaction Groups/Reporter Groups & Notivisa \% (n) & VigiBase \% (n) & EudraVigilance \% (n) \\
\hline General disorders and administration site conditions & $45.5(107)$ & $17.5(6564)$ & $15(3992)$ \\
\hline Vascular disorders & $12.3(29)$ & $3.3(1220)$ & $3.2(721)$ \\
\hline Gastrointestinal disorders & $6.4(15)$ & $8.9(3352)$ & $7.3(1663)$ \\
\hline Musculoskeletal and connective tissue disorders & $6.4(15)$ & $4.9(1825)$ & $3.8(869)$ \\
\hline Skin and subcutaneous tissue disorders & $6.4(15)$ & $6.1(2299)$ & $5.5(1247)$ \\
\hline Cardiac disorders & $5.5(13)$ & $7.6(2873)$ & $10.4(2356)$ \\
\hline Respiratory, thoracic and mediastinal disorders & $5.5(13)$ & $7.3(2737)$ & $9.3(2098)$ \\
\hline Nervous system disorders & $3.8(9)$ & $6.8(2543)$ & $6.2(1410)$ \\
\hline Blood and lymphatic system disorders & $1.3(3)$ & $5.9(2229)$ & $5.0(1139)$ \\
\hline
\end{tabular}

\section{Brazilian Regulation in Drug Surveillance}

\author{
P.C. Mastroianni ${ }^{1}$, T. Herdeiro ${ }^{2,3}$, F. Roque ${ }^{2,4}$, F.R. Varallo ${ }^{\mathbf{1 , 5}}$
}

${ }^{1}$ São Paulo State University), School of Pharmaceutical Sciences, Araraquara, Brazil, ${ }^{2}$ Institute for Biomedicine, Medical Sciences Department, University of Aveiro, Aveiro, Portugal, ${ }^{3}$ Institute of Research and Advanced Training in Health Sciences and Technologies, Gandra, Portugal, ${ }^{4}$ Research Unit for Inland Development, Guarda Polytechnic, Guarda, Portugal, ${ }^{5}$ Américo Brasiliense Hospital, Araraquara,

Introduction. Brazilian health legislation on drug surveillance has been available for only four decades [1]. Pharmacovigilance actions are constantly changing, adding resources to proactively detect drug-related problems. Prevalence data obtained on post-marketing surveillance have been used to identify genetic biomarkers [2,3], aiming to improve the safety and effectiveness of the pharmacotherapy [3].

Objective: to assess the Brazilian legislation on pharmacovigilance, in order to identify the conceptual changes and actions in drug post-marketing surveillance over the years.

Methods: A critical review of the legislations and communications published between 1976 and 2017 was conducted. Sixty-two documents were identified.

Results: Advancements in the assessment of health technologies occurred only after the publication of the National Drug Policy (1998), the foundation of the National Health Surveillance Agency (Anvisa), and the creation of the National Center for Drug Monitoring (2001). From 2009, pharmacovigilance practices became mandatory for marketing authorization holders. Regulatory advancements in pharmacovigilance in Brazil are equivalent to international practices. However, there is still a lack of regulations for biosimilars and veterinary medicines, of agility in reporting non-serious risks to manufacturers and health care services, and of encouragement for reporting technical complaints and quality deviations, which could improve and control post-marketing drug quality

Conclusion: It is necessary to encourage and to develop strategies for technical complaint and quality deviation reporting, in order to improve and control post-marketing drug quality. Also, there is a need for decentralization, leading to a higher equity between Brazilian regions in regards to risk communication related to the use of drugs.

Further sources of information/References:

1. Mastroianni PC, Varallo FR,Hernandes CD,. Brazilian Regulation in Pharmacovigilance: A Review. Pharmaceut Reg Affair.2016; 5:1000164

2. Awada Z, Zgheib NK. Pharmacogenovigilance: A pharmacogenomics pharmacovigilance program. Pharmacogenomics. 2014; 15: 845-56

3. Blankstein S. Pharmacogenomics: history, barriers, and regulatory solutions. J Food Drug Law 2014; 69: 273-314

\section{Adverse Events Following Immunization: Injection Site Abcesses}

F. Ben Salem ${ }^{1}$, S. Kastalli ${ }^{1}$, M. Bouhlel ${ }^{1}$, R. Daghfous ${ }^{1}$, S. El Aidli $^{1}$, A. Zaiem ${ }^{1}$

${ }^{1}$ Tunsian National Centre Of Pharmacovigilance, Tunis, Tunisia 
Introduction: The adverse events following immunization (AEFI) can be local or general reactions. The local reactions include erythema, swelling, hematoma, abscess, or cellulitis. For the general reactions, we can mention fever, tiredness, shivers, headaches or myalgias. These reactions are generally mild and transient. Serious reactions are extremely rare. The benefits of preventing disease far outweigh the risks of immunization.

Aim: To analyze AEFI including injection site abcesses notified to the Tunisian National Centre of Pharmacovigilance.

Methods: It is a retrospective study which included all the observations of adverse events of injection site abcesses notified during 2015. The imputability was assessed according to the imputability method of OMSUMC.

Results: It was about 24 patients. Sex ratio M/W was 1.4. Median age was 3 months. Vaccines were administered in association. Injection site was deltoid in 4 cases and thigh in 22 cases. Two cases received 2 injections respectively in the deltoid and the thigh. Pentavalent vaccine (hepatitis B, diphtheria, tetanus, pertussis, haemophilus type b) was the most incriminated (in 19 cases). The delay of appearance of manifestations varied from one day to 3 months. BCGitis were found in 4 infants and abscess in the thigh in 22 infants. Seventeen among the last ones presented signs of hot abscess including local heat, swelling, pain, and skin fistulisation. An ultrasound was made at 3 infants with results in favor of hematoma of the thigh. Treatment was laying flat abcess associated or not to antibiotic therapy or 10 cases and only antibiotic therapy for 4 cases. The evolution was favorable.

Discussion: The infectious origin of these abscesses was suspected in 17 cases and an error in the injection technique in 3 cases. In the literature, swellings were the most frequent local AEFI. Inflammatory signs can be associated with these swellings because of defect asepsis. Hematoma can be formed when the femoral artery are affected in case of injection in the lateral thigh seen its closeness of the neurovascular beam. Local reactions even ulcerations are usually occurred with BCG with recovery in few months.

Conclusion: Local reactions are not a contraindication to further doses of vaccines. We recommend insuring the formation of vaccinators to improve the techniques of vaccines injection in order to reduce the incidence of these AEFI.

\section{Safety and efficacy of different hyperkalaemia pharmacotherapies and their use in distinct clinical conditions}

P.C. Mastroianni ${ }^{1}$, V. Trombotto ${ }^{1}$, R.C. Lucchetta ${ }^{2}$, T.

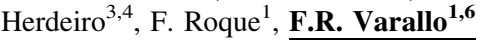

${ }^{1}$ São Paulo State University, School of Pharmaceutical Sciences, Araraquara, Brazil, ${ }^{2}$ Federal University of Paraná, Pharmacy, Curitiba, Brazil, ${ }^{3}$ Institute for Biomedicine, Medical Sciences Department, University of Aveiro, Aveiro, Portugal, ${ }^{4}$ Institute of Research and Advanced Training in Health Sciences and Technologies, Gandra, Portugal, ${ }^{5}$ Research Unit For Inland Development, Guarda Polytechnic, Guarda, Portugal, ${ }^{6}$ Américo Brasiliense Hospital State and Universidade Paulista, Araraquara, Brazil

Introduction: There are several options of drugs used for the treatment of hyperkalaemia, therefore it is necessary to analyse their safety and effectiveness in order to assess the risk-benefit balance among them. [13]

Objective: To evaluate the efficacy and safety of hyperkalaemia pharmacotherapy.
Methods: A systematic review was performed in the databases Medline, LILACS and Cochrane Library since its insertion date in which fifty-six publications were identified. There were included fourteen eligible randomized clinical trials, cohort and case control studies, that evaluates the safety, efficacy and/or effectiveness of the pharmacotherapy used in hyperkalaemia treatment, compared with treatment absence, placebo or other drugs. To assess the quality and risk of bias of the clinical trials will be used the the Cochrane Collaboration tool and to assess observational studies will be used the NewCastle-Ottawa scale. The protocol was submitted in 26 April 2017 for registration in PROSPERO.

Results: Fourteen different medicines were included whom the administration could be infusion, "in bolus", nebulised or oral. The serum potassium was reduced from 1 to $3 \mathrm{mEq} / \mathrm{L}$ in the following interventions: salbutamol (from 2.5 to $5 \mathrm{mg}$-nebulised), sodium-bicarbonate $8.4 \%$ (50 $\mathrm{mL})+$ salbutamol $(0.5 \mathrm{mg})$; salbutamol $(0.5 \mathrm{~g})+$ insulin $(10 \mathrm{UI})+$ dextrose $5 \%(50 \mathrm{~mL})$; albuterol (400 ug-nebulised); sodium-bicarbonate $8,4 \%$ (3 ampoules $)+$ insulin $(20 \mathrm{UI})+$ dextrose $10 \%(1000 \mathrm{~mL})$ and sodium $(30 \mathrm{~g}) \mathrm{e}$ calcium $(5 \mathrm{~g})$ polystyrene sulfonate. Most common adverse events reported were gastrointestinal disorder, edema, anorexia $(1,2)$, cough, hypokalaemia, hypocalcemia e hypomagnesemia. Sodium-bicarbonate $8.4 \%(50 \mathrm{~mL})$, glucose $50 \%(100 \mathrm{~mL})$ and insulin (10 UI) + glicose $40 \%$ $(100 \mathrm{~mL})$ were identified as less effective.

Conclusion: Preliminary data suggest that salbutamol is effective in patients with chronic and acute Renal Failure (RF) (infusion) including in children with RF children (nebulised). Albuterol administration is suggested to premature neonates with RF In dialysis patients the use of fludrocortisone $(0.1 \mathrm{mg})$ indicates to be an effective alternative in chronic use. In cases of patients with the comorbidities: chronic renal disease, dyslipidaemia, hypertension, diabetes, coronary artery disease, history of stroke, arrhythmia and congestive heart failure the sodium polystyrene sulfonate $(30 \mathrm{~g})$ has been shown to be effective despite reported adverse events.

Further sources of information/References:

1. [1]. Rossignol P, Legrand M, Kosiborod M, Hollenberg SM, Peacock WF, Emmett M, et al. Emergency management of severe hyperkalemia: Guideline for best practice and opportunities for the future. Pharmacol Res 2016;113:585-91

2. [2]. Neto OMV, Neto MM. Distúrbios do equilíbrio hidroeletrolítico. Medicina (B Aires). 2003;36:325-37

3. [3]. Pitt B, Bakris GL. New Potassium Binders for the Treatment of Hyperkalemia. Hypertension. 2015;66:731-8

\section{Hypersensitivity reaction associated with intravenous administration of folinic acid in patient with gastric cancer}

\author{
C. Ladhari ${ }^{1}$, R. Sahnoun ${ }^{1}$, A. Zaiem ${ }^{1}$, F. Ben Salem ${ }^{1}$, S. El Aidli ${ }^{1}$, \\ R. Daghfouss ${ }^{1}$, S. Kastalli ${ }^{1}$
}

${ }^{1}$ National Pharmacovigilance Centre, Tunis-bab Souika, Tunisia

Background: Five-fluorouracil (5-FU) is used in many chemotherapy protocols for digestive cancers and others cancers. In oncology, to enhance the 5-FU cytotoxic effects, folinic acid is usually added. Adverse events of folinic acid have rarely been reported in the literature [1] .

Methods: We report a case of anaphylactic reaction to folinic acid in a patient with gastric cancer 
Results: A 52-year-old woman was diagnosed with gastric cancer since September 2015. She underwent a FOLFOX chemotherapy regimen (5FU+ Folinic acid+ oxaliplatin). At the 7th cure, during perfusion of oxaliptlatin and folinic acid, she developed tingling of hands and sickness. The perfusion was stopped and she was given intravenous hydrocortisone. Since it was felt that the allergic reaction could be related to a hypersensitivity reaction to oxaliplatin, we had recommended premedication with prednisolone before the next cure. At the $8^{\text {th }}$ cure, she developed facial flushing and pruritis at the end of oxaliplatine + folinic acid perfusion. It was then decided to stop oxaliplatin and to adopt the LV5FU2 protocol (5FU+ Folinic acid). On the first day of the new regimen, $10 \mathrm{~min}$ after the Y-set infusion of folinic acid and 5FU, the patient developed again facial flushing and pruritis. The infusion was stopped. She received symptomatic treatment. A fourth reaction occurred on March 16, 2016, whereas the infusion of folinic acid was starting, the patient felt hot and presented a generalized urticaria. The infusion was then stopped.

Discussion: The responsibility of the folinic acid was retained in front of: the suggestive delay of the onset of the reaction few minutes after beginning of folinic acid, the favorable evolution after the withdrawal of infusion, and especially the recurrence of the reaction 4 times among them one with folinic acid alone.

Conclusion: Folinic acid may be a rare cause of anaphylactic reaction occurring during chemotherapy administration.

Further sources of information/References:

1. Katirtzoglou NA, et al. Anaphylactic reaction associated with intravenous administration of folinic acid in a patient with colon cancer. In Vivo 2011; 25: 995-6

\section{Impact of Internet and Social Media Use in Pharmacovigilance}

\author{
O. Pinar ${ }^{1}$, N. Guresci ${ }^{1}$, B. Unal ${ }^{1,2}$ \\ ${ }^{1}$ Bayer Turk Kim. San. Ltd. Sti., Istanbul, Turkey, ${ }^{2}$ Marmara \\ University, Faculty of Pharmacy, Toxicology Department, \\ Pharmacogenetics and Drug Safety Unit, Istanbul, Turkey
}

Introduction: With the recently increased use of internet and social media, consumers have started searching for information about health and drugs, getting in touch with other consumers and sharing their experiences on these platforms [1].Social media use increased from $8 \%$ in 2005 to $67 \%$ in 2012, and Facebook has been found to be the 4th most popular source for health related information in United Kingdom.

Objective: Health related information and posts shared by patients via social media may contain new safety information. Thus, its relevance on pharmacovigilance systems and its coverage in regulations of Turkish Drug and Medical Device Agency (TITCK) and European Medicines Agency (EMA) have been evaluated.

Method: Regarding this new situation, harmonization has been initiated for report collecting rules and processes in the field of pharmacovigilance. The "Guideline on Good Pharmacovigilance Practices (GVP) Module 1Management and reporting of adverse drug reactions" issued by Turkish Drug and Medical Device Agency on 12 June 2014 and the "Guideline on Good Pharmacovigilance Practices (GVP) Module 6-Management and reporting of adverse reactions with medicinal products" updated by European Medicines Agency on 8 September 2014 include the rules of processing information about suspected adverse reactions obtained from internet or digital platforms [2,3]. In our present study, the relevant regulations have been evaluated and compared.
Conclusion: The relevant section of the Guidelines on Good Pharmacovigilance Practices (GVP) Module 1 issued by Turkish Drug and Medical Device Agency and the Guideline on Good Pharmacovigilance Practices (GVP) Module 6 updated by European Medicines Agency have been compared and found to be consistent. The digital platforms under the responsibility of companies are regularly screened and the reports meeting the relevant criteria are processed in line with national and international reporting rules. Additionally, "active online listening" is performed for the internet platforms which are not under the control of companies. Active online listening is the process of following, analyzing and interpreting digital interactions, communications and subject matters of internet users. The reports obtained from active online listening are subject to evaluation, and cases considered as adverse events are processed as necessary. During the 8-year period from 2002 to 2010 , there were 9 publications on detecting adverse events in social media and in 2014 this figure dramatically increased to 23 articles, these publications have been reviewed [4] In this field, the availability of a vast amount of information in social media increases the interest in monitoring adverse drug reaction data [5]

Internet can be an interesting source of data about adverse drug reactions. Marketing authorization holders and health professionals should pay more attention to patients concern and impacts on daily life. Data from patient websites also could be used as a source of data to detect adverse events in pharmacovigilance. Identifiable patient and reporter criteria clarifications regarding the quality and content of reports and updates are warranted. However, social media also presents unique challenges due to brevity, lack of structure and informal language. Because of the website configuration and limited information, it is not possible to verify if the events meet the reporting criteria. Nevertheless, patients share real life experiences about common adverse drug reactions but also unexpected adverse drug reactions that should not be neglected.

Further sources of information/References:

1. Edwards R, et al. Social Media and Networks in Pharmacovigilance Boon or Bane? Drug Saf 2011; 34 : 267-271

2. Turkish Drug and Medical Device Institution Guideline on Good Pharmacovigilance Practices (GVP) Module I-Management and reporting of adverse drug reactions (12.06.2014)

3. European Medicines Agency. Guideline on Good Pharmacovigilance Practices Module 6-Management and reporting of adverse reactions with medicinal products $(08.09 .2014)$

4. Golder S, et al. Systematic review on the prevalence, frequency and comparative value of adverse events data in social media. Br J Clin Pharmacol 2015; 80:878-88

5. A. Sarker et al. Utilizing social media data for pharmacovigilance: A review. J Biomed Inform 2015; 54:202-12

\section{Safety profile of fractional dosing of yellow fever vaccine, experience from community based vaccine pharmacovigilance in Kinshasa}

\author{
D. Nzolo Bomene ${ }^{\mathbf{1 , 2 , 3}}$, A. Engo Biongo ${ }^{1,3}$, M. Lusakibanza ${ }^{1,3}$, \\ C. Nsibu Ndosimao $^{1,3}$, G. Tona Lutete ${ }^{1,3}$, J.P. Van geertruyden ${ }^{2}$
}

${ }^{1}$ Unit Of Clinical Pharmacology and Pharmacovigilance, Kinshasa, Democratic Republic of the Congo, ${ }^{2}$ University of Antwerp, Antwerp, Belgium, ${ }^{3}$ National Pharmacovigilance Center, Kinshasa, Democratic Republic of the Congo

Background: In 2016, there were a yellow fever (YF) outbreak in Central Africa with several YF confirmed and death cases reported in Angola and 
Democratic Republic of Congo (DRC). Due to shortage in vaccine supply, fractional dosing was proposed in preventive vaccination campaign in August 2016 in Kinshasa where $0.1 \mathrm{ml}$ were of Yellow Fever Vaccine was administered in a population of 12 million inhabitants. Pregnant women and children under 2-year-old received $0.5 \mathrm{ml}$. A pharmacovigilance system was organized to track adverse events following immunization (AEFI)

Aim: To described most reported AEFIs, as captured through community based pharmacovigilance.

Methods: Active pharmacovigilance was organized in churches, student dormitories as well as in pediatrician, obstetrician and friend networks. Oral discussions, phone calls, SMS and what's app messages were the main communication tools. Education about AEFI was provided before data collection. AEFI were captured in specific ICSRs, entered into Vigiflow and extracted for descriptive analysis using Stata 12. WHO-ART were used for AEFI description

Results: From the population who received fractional dose, 4020 subjects reported 5918 AEFI (1.5 AEFI per subject). Reporters were mostly males (54.9\%) with an average of $26 \pm 10.7$-year-old. Median time to onset was 1 day (IQR: 1, 1) and median duration of events was 2 days (IQR: 1, 3). Most reported SOCs were body as a whole-general disorders $(56.9 \%$ of subjects), neurological disorders (32.2\%) and Gastrointestinal disorders (24.9\%). Most reported PTs were fever (34.7\%), headache (19.9\%) and diarrhea $(11.1 \%)$. Most reported local events were injection site pain (10\%), Numbness localized (6.0\%) and injection site reaction (1.2\%). Six cases were serious and included one fatal case of suspected yellow fever following vaccination in a child with malnutrition and tuberculosis and one case of vaginal hemorrhage and abortion.

Conclusion: Community based pharmacovigilance is an effective tool to monitor vaccine safety during mass immunization campaigns. Fractional dosing of yellow fever vaccine is safe but should be administered with caution in pregnant women and immunocompromised subjects, to whom normal dose is already contraindicated.

\section{Evolution of the Western Cape Antiretroviral Pharmacovigilance Programme in South Africa}

K. Cohen ${ }^{1}$, J. Jones ${ }^{1}$, A. Stewart ${ }^{2}$, J. Voget ${ }^{3}$, T. Naledi ${ }^{3}$,

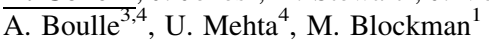

${ }^{I}$ Division Of Clinical Pharmacology, Department Of Medicine, University of Cape Town, Cape Town, South Africa, ${ }^{2}$ Clinical Research Centre, University of Cape Town, Cape Town, South Africa, ${ }^{3}$ Western Cape Department of Health, Cape Town, South Africa, ${ }^{4}$ School of Public Health and Family Medicine, University of Cape Town, Cape Town, South Africa

Introduction: In 2005, when the South African antiretroviral treatment (ART) programme commenced, the Western Cape Province Department of Health and the Division of Clinical Pharmacology at the University of Cape Town established a targeted spontaneous reporting system for suspected serious adverse drug reactions (ADRs) in patients receiving ART. Goals were to: (1) Identify signals of new ADRs and/or ADR patterns, (2) Identify risk factors, (3) Inform treatment policy to minimize the risk of harm to patients from ADRs, and (4) Promote drug safety awareness among healthcare workers (HCWs). The programme was expanded to include ADRs in patients receiving tuberculosis (TB) treatment in 2012. Aim: To describe ADR reports received since 2005 and explore changes in ADRs reported over time.

Methods: Reports are submitted using a standardised reporting form which includes case definitions of priority ADRs. The system solicits reports of suspected ADRs that are fatal, life-threatening, result in hospitalisation, prolong hospitalisation, cause disability and/or result in cessation of the implicated drug. We compared pattern of ADRs reported before 2012, and from Jan 2012 onwards.

Results: There were 3463 events reported, an average of 315 per year, of which $85 \%$ were assessed to be ADRs. Doctors submitted $74 \%$, nurses $17 \%$ and pharmacists $8 \%$ of reports overall. Proportion submitted by nurses dropped from 18\% before 2012 to $13 \%$ from 2012 onwards. A higher proportion of reported ADRs were in female patients in the period before 2012: $70 \%$ versus $57 \%$ female $(\mathrm{p}<0.0001)$. The 3 most common ADRs reported before 2012 were hyperlactataemia (490 cases), lipodystrophy (387 cases), and paraesthesia/peripheral neuropathy (335 cases); stavudine was the most frequently implicated drug in these ADRs. The 3 most common ADRs reported from 2012 onwards were renal dysfunction (223 cases, tenofovir and aminoglycosides most frequently implicated), rash (145 cases, efavirenz and cotrimoxazole most frequently implicated) and lipodystrophy (139 cases, stavudine most frequently implicated).

Conclusions: The pattern of reports received reflects key safety concerns with ART regimens in use at the time. Before 2012, ADRs caused by stavudine predominated. From 2012 onwards renal dysfunction was the most commonly reported ADR, with tenofovir most frequently implicated. Drugs used in management of opportunistic infections were implicated in ADRs occurring in patients on ART. ADR reports were predominantly submitted by doctors. With increasing focus on nurse-based care in the ART programme, encouraging reporting of ADRs by nurses, and supporting nurses in diagnosing and managing ADRs is a priority.

\section{Unexpectedly High Fatality Rate in Globally Collected Spontaneous Reports of Progressive Multifocal Leukoencephalopathy with Alemtuzumab}

\author{
M. van $\operatorname{Eijk}^{1}$, O. Caster $^{2,3}$
}

${ }^{I}$ Utrecht Institute for Pharmaceutical Sciences, Utrecht University, Utrecht, the Netherlands, ${ }^{2}$ Uppsala Monitoring Centre, WHO Collaborating Centre for International Drug Monitoring, Uppsala, Sweden, ${ }^{3}$ Department of Computer and Systems Sciences, Stockholm University, Kista, Sweden

Introduction: Alemtuzumab is an immunomodulating monoclonal antibody $(\mathrm{mAb})$ targeting the CD52 antigen of lymphocytes. Its indications are B-cell chronic lymphocytic leukaemia and relapsing-remitting multiple sclerosis, but uses include other immunosuppressive and antineoplastic conditions. Like several other mAbs, alemtuzumab has been associated with progressive multifocal leukoencephalopathy (PML), a rare but serious demyelinating disease induced by JC virus infection [1]. The characteristics of alemtuzumab-associated PML, including its fatality rate, are largely unknown.

Aim: To identify and describe the most prominent characteristics of globally collected spontaneous reports on alemtuzumab with PML.

Methods: Data were taken from VigiBase, the WHO global database of individual case safety reports, as of 15 September 2016, after exclusion of suspected duplicates [2] and reports with HIV/AIDS treatment. Alemtuzumab reports with PML were compared to (a) PML reports for non$\mathrm{mAb}$ drugs, and (b) to alemtuzumab reports with other suspected reactions than PML, with respect to reporting rates of numerous different characteristics including patient age and sex, fatality, and co-reported drugs and adverse reactions. Analysis was performed with vigiPoint, a method that identifies reporting characteristics deviating significantly between a foreground of reports and a suitable reference group of reports, in terms of log odds ratios [3]. 
Results: Fatality occurred in 31 cases $(77 \%)$ for alemtuzumab/PML, compared to $36 \%$ for non-mAb drugs reported with PML, and $23 \%$ for non-PML alemtuzumab reports. Among those fatal cases with data available, $78 \%$ of patients were male and the median age was 68 years. The reported alemtuzumab indication was leukaemia in 21 cases and multiple sclerosis in one case. For reference, rituximab had the second highest PML fatality reporting rate among the mAbs at $46 \%$. A second main characteristic of alemtuzumab PML reports was co-reporting of rituximab, another $\mathrm{mAb}$ used in leukaemia. This occurred in 17 cases (43\%), compared to $2 \%$ for non-mAb drugs reported with PML, and 5\% for alemtuzumab non-PML reports. $\Omega$ for alemtuzumab-rituximab-PML was 1.8 (95\% CI 1.1-2.5), which indicates significantly higher reporting than expected [4].

Conclusions: In VigiBase, the proportion of fatal cases in alemtuzumabassociated PML is high both in absolute and relative terms. The typical patient is male, above middle age, and treated for leukaemia. Concurrent use of alemtuzumab and rituximab may synergistically add to the PML risk, although this hypothesis warrants independent verification. These results refer to reporting patterns, and causality has not been assessed. Fatality rates in spontaneous reports are expected to be inflated due to differential under-reporting.

Further sources of information/References

1. Ferenczy MW, Marshall LJ, Nelson CD, Atwood WJ, Nath A, Khalili $\mathrm{K}$, et al. Molecular biology, epidemiology, and pathogenesis of progressive multifocal leukoencephalopathy, the JC virus-induced demyelinating disease of the human brain. Clin Microbiol Rev 2012; 25:471-506

2. Norén GN, Orre R, Bate A, Edwards IR. Duplicate detection in adverse drug reaction surveillance. Data Min Knowl Discov 2007; 14 : 305-28

3. Juhlin K, Star K, Norén GN. Pinpointing Key Features of Case Series in Pharmacovigilance-a Novel Method. Drug Saf 2013; 36: 912-3

4. Norén GN, Sundberg R, Bate A, Edwards IR. A statistical methodology for drug-drug interaction surveillance. Stat Med 2008; 27: 3057-70

\section{Activity Report of the Pharmacovigilance Unit of the Mohammed V Military Instruction Hospital Rabat Morocco "May 2016-March 2017"}

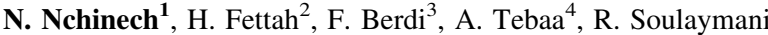
Bencheikh $^{5}$, Y. Bousliman 6

\begin{abstract}
${ }^{1}$ Faculty of Medicine and Pharmacy-Mohamed V University, Pharmacy Department-Mohammed V Military Teaching Hospital, Rabat, Morocco, ${ }^{2}$ Faculty of Medicine and Pharmacy-Mohamed $V$ University, Pharmacy Department-Mohammed V Military Teaching Hospital, Rabat, Morocco, ${ }^{3}$ Faculty of Medicine and PharmacyMohamed V University, Pharmacy Department-Mohammed V Military Teaching Hospital, Rabat, Morocco, ${ }^{4}$ Centre Anti Poison et de Pharmacovigilance du Maroc, Rabat, Morocco, ${ }^{5}$ Faculty of Medicine and Pharmacy-Mohamed V University, Centre Anti Poison et de Pharmacovigilance du Maroc, Rabat, Morocco, ${ }^{6}$ Faculty of Medicine and Pharmacy-Mohamed V University, Pharmacy Department-Mohammed V Military Teaching Hospital, Rabat, Morocco
\end{abstract}

Background: In order to promote the spontaneous reporting of adverse reactions within the Mohammed V Military Instruction Hospital (HMIMV) and sensitize patients and health professionals, a
Pharmacovigilance Unit (PVU) was created in May 2016 at the pharmacy department of this institution following the appointment of a referent Pharmacist in Pharmacovigilance succeeding the advent of ministerial circular 003 of 04/01/2016 on The organization of the national pharmacovigilance system.

Aim: To evaluate 10 months of activity of this Unit with the services of our establishment.

Material and Method: This is a retrospective descriptive study of the reports emanating from the various clinical departments as well as those received from cancer patients during the dispensation of their treatments in the Functional Unit for the Management of Products with Special Status of the Pharmacy department (FUMPSS), Conducted from 01 May 2016 to 01 March 2017. The reporting was collected on the Reporting Cards for Adverse Events to Medicines, Vaccines and Other Health Products provided by the Anti Poison Center and Pharmacovigilance of Morocco.

Results: Since May 2016, the PVU has collected 123 notifications of which $37 \%$ were related to the adverse effects of cytotoxic drugs, followed by hormone therapy with $27 \%, 4$ notifications were reported following the use of antituberculosis drugs (3.3\%), and $5.7 \%$ To the use of medical devices ( 7 notifications). The severity of reported cases varies from mild cases such as fatigue or diarrhea to very severe cases such as: 5 cases of toxidermia including 3 Lyell syndromes and 1 case of pulmonary embolism. The reports were distributed as follows: $75 \%$ active and $25 \%$ spontaneous. The majority of reports were received from the FUMPSS with 67 notifications (54.5\%). The first clinical reporting medical service was the Oncology Department for Pharmacovigilance with approximately $7 \%$ (9 cases) and the Cardiovascular Surgery Department for Materiovigilance with approximately 5\% (6 cases). A study of the causes of underreporting [1] (was carried out among 50 doctors at the institution, who mainly attributed the absence of feedback $(50 \%)$ and the workload (46\%).

Conclusion: The results of this review enabled us to undertake a number of corrective actions in the face of serious and frequent problems such as the intensification of pharmaceutical interviews for the benefit of cancer patients and the organization of awareness campaigns for hospital practitioners, the importance of notification, including the 1st scientific day of Pharmacovigilance of HMIMV.

Further sources of information/References

1. Berdi F, Nchinech N, Tadlaoui Y, Ziraoui W, Bennana A, Bousliman Y. Evaluation of the reasons for the under-reporting of pharmacovigilance cases at the Mohammed V Military Instruction Hospital Rabat. $10^{\text {th }}$ National Pharmacovigilance Congress, Rabat, 22 \& 23 December 2016

\section{Baclofene safety and its use in social media: a preliminary study.}

A. Lillo Le Louet ${ }^{1}$, R. Aboukhamis ${ }^{1}$, P. Karapentiaz ${ }^{2}$, M. Lemoine $^{1}$, P. Zweigenbaum ${ }^{3}$, D. Leprovost ${ }^{4}$, C. Bousquet ${ }^{4,5}$

${ }^{1}$ Federation de Pharmacovigilance Ile De France, Paris, France, ${ }^{2}$ INSERM UMRS 1138 team 22, Paris, France, ${ }^{3}$ LIMSI CNRS, Saclay, France, ${ }^{4}$ INSERM U872 EQ20, Paris, France, ${ }^{5}$ DSPIM, Saint Etienne, France

Background: Health professionals, authorities as well as pharmaceutical industries have been interested in health-related comments on social media as potential new source of knowledge in pharmacovigilance to analyze drug use, abuse and misuse, as well as adverse drug reactions (ADR). As part of a public funding from the National Agency for Drug Safety, a 
multidisciplinary team involving Pharmacovigilance Unit $(1,2)$, Medical Informatics and Public Health $(3,6)$, Information and language (4) and Semantic web (5), have been working since 2013 on the Vigi4MED project. Tools to extract information from selected website, perform a language analysis and evaluate semantic relation as potential ADR between a drug and a symptom were developed. We present here preliminary results on one case study.

Aim: evaluate the input of social media on information about ADR and drug misuse.

Material and methods: Baclofen has been a mainstay in the management of spasticity in several neurological diseases since the mid-70s. The offlabel use of high-dose baclofen (HDB) for alcohol-dependence has spread attention in France in the last 5 years. Due to the rapid increase of this prescribing practice, the French health authorities have decided to frame it using an extraordinary regulatory measure named "temporary recommendation for use". Recent signals emerged on other off-label use of baclofen made this drug a perfect subject for this case study. We performed a retrospective, observational and descriptive study, based on selected forum discussions mentioning baclofen. All baclofen messages were manually reviewed by two trained pharmacist (RA and ML) using a special tool (DL). Potential ADR were evaluated as expected or unexpected.

Results: from January 2013 to December 2014, all discussion involving baclofen were selected, and 18,750 messages were extracted. Due to this huge number we excluded all baclofen discussions related to alcohol and focused on other indications (anorectic, drug abuse withdrawal) and potential ADRs. 2621 messages were individually reviewed and 782 (4\% of the total) were annotated as potential ADRS. Misuse of baclofen to lose weight, and in illicit drug abuse (mainly cocaine and cannabis) were found. Potential unexpected ADRs were evaluated using a semi-automated tool and only a few cases were found, needing further evaluation.

Conclusion and perspective: this case study shows that discussions from selected websites contain information about drug use and potential ADRs. Nevertheless, to date, it appears that the relevant data for PV is probably low and a manual and human process is necessary.

\section{Pharmacovigilance and Patients: How to Improve Reports' Quality?}

\section{A. Lillo Le Louet ${ }^{1}, \mathrm{H}$. Barillier ${ }^{1}$, C. Le Bellet ${ }^{1}, \mathrm{H}$. Le Louet $^{1,2}$ \\ ${ }^{I}$ Fédération De Pharmacovigilance Ile De France, Creteil, France, ${ }^{2}$ Fédération De Pharmacovigilance Ile De France, Creteil, France}

Background: according to the European Directive, the French legislation has allowed the patients and organisations representing consumers to report adverse drug reactions (ADR) to the pharmacovigilance (PV) centers since June 2011. In France, a specific form is available for patient's report in the Agency website. In the Ile de France area, a Pharmacovigilance website has been available for the last 4 years, and was designed with a specific space for patients. The latter can also declare directly by phone, letter, or email. All patients reporting an ADR receive a direct answer from the PV unit, mainly a letter with patient's history and description of the ADR, a general presentation of the data available about the ADR reported, and a short conclusion regarding the patient's case. Depending on the ADR characteristics (mainly seriousness and unexpectedness), the answer is sent within 6 weeks after its reception by the PV unit and its registration in the national PV database.

Aim: in order to improve the relations between patients and pharmacovigilance units, we assess the patient's expectations regarding their reports management, as well as their satisfaction regarding the PV feedback.

Material and methods: All patients' reports during a six months period, from October $15^{\text {th }} 2016$ to April $15^{\text {th }} 2017$, were selected. To assess the patient's expectations and feedback after our answers, a special questionnaire was elaborated for this study. We investigate on three lines: patient's motivation to report ADR; technical and practical aspect of ADR report; and response quality assessment. We used a closed-answer questionnaire using a Likert scale, but open answers were also possible for some questions.

Preliminary results: from the six months period, a total of 778 reports were received, and 59 came from patients (8.6\% of the total). 7 cases were excluded, either because they came from specific patient's association about DES use $(n=6)$, or because the reporter had no valid mail $(n=1)$. The main characteristics of these 52 reports were: patient's report for themselves $(80 \%)$, from women $(80 \%)$, and the reports were mainly non serious (79\%). Half of the reports came from websites (51\%). We sent our questionnaire to all patients, and preliminary results showed that the main reasons for consumers to report were the severity of reactions, and their concerns about their situation; they were globally satisfied with the received feedback.

Discussion and Perspectives: we would like to share the questionnaire with the overall PV unit of the Ile de France area. We will also complete this survey sending prospectively the questionnaire to all patients' reports in the next months.

\section{The Effect of Concomitant Antiepileptic Drugs on Plasma Lamotrigine Concentration}

\author{
F. Ben Salem ${ }^{1}$, E. Gaies ${ }^{1,2}$, N. Jebabli ${ }^{1,2}$, I. Salouage ${ }^{1,2}$, \\ R. Charfi ${ }^{1,2}$, H. El Jebari ${ }^{1,2}$, A. Klouz ${ }^{2}$, R. Daghfous ${ }^{1,2}$, \\ S. Trabelsi $1^{1,2}$
}

${ }^{1}$ Department of Clinical Pharmacolgy-National Centre of Pharmacovigilance, Tunis, Tunisia, ${ }^{2}$ Laboratoire de recherche en Pharmacologie Clinique et expérimentale, Tunis, Tunisia

Introduction: Lamotrigine (LTG) is one of the newer antiepileptic drugs (AED). Its association to other AEDs can lead to a reduce or an increase of LTG concentration because of drug interactions involving induction or inhibition of LTG metabolism [1].

Aim: To evaluate the influence of AEDs on LTG bioavailability.

Methods: It is a retrospective study (Mai 2012-Feb 2017) including 374 samples of trough LTG concentration (C0) obtained from 280 patients. Our population was divided into four groups: the 1st group included those treated by LTG on monotherapy $(\mathrm{n}=293)$, the $2 \mathrm{nd}(\mathrm{n}=19)$ and the 3rd groups $(n=46)$ those who received bitherapy with respectively enzymatic inducers (Phenobarbital $(\mathrm{Pb})$ or Carbamazepine (CBZ)), and enzymatic inhibitors (valproic acid (VA)) and the 4th group was under LTG associated to AEDs without a documented effect on drug interactions (levetiracetam and pregabalin $(\mathrm{n}=16)$ ). Monitoring of LTG was made using immunoassay method (INDIKO ${ }^{\circledR}$ ). Therapeutic interval of LMG C0 was $3-15 \mathrm{ug} / \mathrm{mL}$. The ratio $\mathrm{C} 0 /$ dose was calculated for all groups and comparison was made using test $\mathrm{U}$ of Mann Witeny and Kruskall Wallis test.

Results: the median age was 12.42 years and the Sex ratio M/W was 1.07 . The distribution of LTG $\mathrm{C} 0$ according to therapeutic interval for each group is summarized in table1.

The correlation between dose and $\mathrm{C} 0$ was 0.302 in monotherapy, 0.7155 in association with enzymatic inducers and 0.4569 in association with enzymatic inhibitors. The mean ratio $\mathrm{C} 0 /$ dose for the 1 st group was 1.219 
(0.449-1988), for the 2nd group it was $1.06(0.56-1.56)$, for the 3 rd 2.785 $(0.785-4.785)$ and for the 4th $1.222(0.68-1.762)$. There were no significant difference in $\mathrm{C} 0 /$ dose between the 1 st and the 2nd group $(p=0.0582)$. However, there was a significant difference between the 1 st and respectively the $3 \mathrm{rd}$ and the 4 th group $(\mathrm{p}<0.001)$.

Conclusion: The association of enzymatic inducers with LTG seemed to have a trend for decreasing LTG bioavailability. The difference was not significant probably because of the little number of patients included. In fact, $\mathrm{Pb}$ and $\mathrm{CBZ}$ induce cytochrome P450 (CYP) and UGT enzymes [1]. The association to VA having an inhibitor effect on the metabolism of LTG increased significantly the bioavailability of LTG.

Further sources of information/References

1. Yamamoto $\mathrm{Y}$, et al..Influence of concomitant antiepileptic drugs on plasma lamotrigine concentration in adult Japanese epilepsy patients. Biol Pharm Bull 2012; 35: 487-93

\begin{tabular}{llll}
\multicolumn{2}{l}{ Table 1: Distribution of $\mathrm{C}_{0}$ according to therapeutic interval (TI) } \\
\hline & $<\mathrm{TI}$ & $=\mathrm{TI}$ & $>\mathrm{TI}$ \\
\hline Group 1 & $30 \%$ & $69 \%$ & $1 \%$ \\
Group 2 & $79 \%$ & $21 \%$ & $0 \%$ \\
Group 3 & $0 \%$ & $93 \%$ & $7 \%$ \\
Group 4 & $38 \%$ & $62 \%$ & $0 \%$ \\
\hline
\end{tabular}

\section{Adverse Drug Reactions reporting Versus Medication Errors reporting in Public Hospitals using Patient Safety Network system}

\author{
F.A. Al-Braik ${ }^{1}$, M.M. Tashtoush ${ }^{1}$, M.M. Al Ghufli ${ }^{1}$, R.A. $\operatorname{Saad}^{1}$, \\ M.Y. Hasan ${ }^{2}$ \\ ${ }^{1}$ Abu Dhabi Health Services, Abu Dhabi, United Arab Emirates, \\ ${ }^{2}$ College of Medicine \& Health Silences, UAE University, Al Ain, \\ United Arab Emirates
}

Background: Voluntary reporting is considered important for monitoring and assessing adverse drugs reactions (ADRs) and Medication Errors (MEs) in patients. ADRs and MEs can be reported utilizing Patient Safety Network (PSN) system available for healthcare professionals in Hospitals accredited by Joint Commission.

Aim: This study compared incidents of ADRs and MEs reporting by health professionals from government health care facilities in the Emirate of Abu Dhabi, UAE.

Methodology: ADRs and MEs were reported from January 2015 till December 2016. A Multidisciplinary Taskforce was assigned for collecting, assessing causality and evaluating reactions outcomes. The Medication Safety Committee monitored the trend and implemented measures for rational use of medicines.

Results: Total 477 ADR cases were reported of which 251 (52.6\%) were complete. $13(5.18 \%)$ cases were classified definite, $210(83.67 \%)$ probable and $28(11.16 \%)$ possible. 10 medications were commonly involved in 39 ADR cases during 2016 as following: Antibacterial $(n=19,48.7 \%)$, anticancer drugs $(\mathrm{n}=13,33.3 \%)$ non-steroidal anti-inflammatory drugs $(\mathrm{n}=5,12.8 \%)$ and gastrointestinal medications $(\mathrm{n}=2,5.1 \%)$. Hypersensitivity was commonly reported with Antibacterials, shortness of breath and hypersensitivity were reported with chemotherapy agents. $9902 \mathrm{ME}$ reports were submitted during 2015-2016. 10 medications were commonly involved in 473 cases during 2016 as following: Non-steroidal anti- inflammatory such as acetaminophen, diclofenac sodium and ibuprofen $(\mathrm{n}=240,50.7 \%)$, antibacterial medications such as Amoxicillin-Pot Clavulanate, Azithromycin, Cefuroxime $(\mathrm{n}=145,30.7 \%)$, Enoxaprin sodium $(n=41,8.7 \%)$, Xylometazoline $(n=25,5.3 \%)$ and Ergocalciferol $(\mathrm{n}=22,4.7 \%)$.

Discussion: Data generated in this study shown low rate of ADRs reporting compared to MEs [1,2],. That can be explained by the fact that it is not mandatory to report every ADR as compared to MEs despite obligatory implementation of PAN system. Moreover many incomplete ADR report were noticeable. Most of High risk medications were reported as ADRs not MEs due to restricted system for prescription and patients' administration.

Conclusion: It is important to incorporate ADR and MEs reporting in PSN database system that used within accredited hospitals and encourage reporting among different healthcare professionals $[2,3]$.

Further sources of information/References

1. Patsuree A, Krska J, Jarernsiripornkul N. Experiences relating to adverse drug reactions in the community: a cross-sectional survey among patients and the general public in Thailand. Expert Opin Drug Saf 2016; 15:287-95

2. Das BP, Rauniar GP, Bhattacharya SK. Medical errors challenges for the health professionals: need of Pharmacovigilance to prevent. J Nepal Med Assoc 2006; 45:273-8

3. Kavanagh C. Medication governance: preventing errors and promoting patient safety. Br J Nurs 2017; 26:159-165

\section{ADRS and medication errors surveillance after the implementation of an electronic report system}

L. Garza-Ocanas 1 , A. Pérez-Rdz ${ }^{1}$, A. Pérez-Garza ${ }^{1}$, C. González-Nieto $^{1}$, J. Escobedo-Peña ${ }^{1}$, E. Pérez-Rodríguez ${ }^{1}$

${ }^{I}$ Facultad de Medicina y Hospital Universitario Dr. José E. González, San Nicolás de los Garza, México

Introduction: Underreporting of both, Adverse Drugs Reactions (ADRs) and Medication Errors (ME) is a well-documented problem in pharmacovigilance. Analyzing the causes of underreporting at our University Hospital, lack of time to fill reports was one of the main complains of nurses and medical staff. As an intervention strategy for improving the report, an electronic report system for ADRs and ME was implemented in 2013.

Aim: To evaluate ADRs and ME reports after the implementation of an electronic report system.

Methods: An analysis and comparison of spontaneous ADRs and ME reported during 2013 and 2016 at Hospital Universitario "Dr. José E. González", Monterrey, Nuevo León, México was made. The parameters evaluated for ADRs, included: number of reports, patient demographic, drug and reaction characteristics, and reaction severity and outcomes. For ME number, type, consequences, and stage of medication process were evaluated.

Results: A significant increase (350\%) in ME reports was observed after the electronic system implementation with 349 vs 1243 ME in 2013 and 2016 respectively. Most of the ME were in preparation process (2013) and prescription (2016). The most common types of error throughout the medication process were dose, frequency of administration and omission of drug/dose.). No difference in total ADRs reports was observed (61 vs 72). The most noticeable ADRs occurred in skin tissues, with rashes being the most common reactions. The drugs responsible for most ADRs were 
vancomycin, carboplatin and docetaxel (2013) and oncology drug (paclitaxel, oxaliplatin and carboplatin) followed by vancomycin (2016). Most of ADRs had moderate severity. Nurse-pharmacist team reported the most ADRs. A series of factors were considered responsible for ADRs underreporting, including knowledge (nurses) and physicians attitudes.

Conclusions: The electronic system implementation improved the ME reports; bringing to our attention large numbers of $\mathrm{ME}$ of which we were previously unaware, and detecting a change in incidence. No difference was observed in ADRs reports, in spite of the system ADRs signal, indicating lack of nurse-physician monitoring. Training courses for improving skills and knowledge for ADR detection as well as further motivation for involvement of physicians should be considered.

\section{Implementation of an integrative risk management plan for the safe practice of anticoagulants}

\author{
O. Lavon $^{1,2}$, I.R. Fermont ${ }^{1}$ \\ ${ }^{1}$ ISOP Israel,Israel, ${ }^{2}$ Clinical Pharmacology and Toxicology Unit, \\ Carmel Medical Center, Haifa, Israel
}

Background: Anticoagulant medications pose major risks for mortality and morbidity to patients. There is a need for ensuring safe management of these medications. ISOP ISRAEL has designed a method to address risks of anticoagulants, integrating all stakeholders along the whole treatment process, with a system approach and a multidisciplinary risk minimisation plan.

Aim: To implement an integrative risk management program for the use and practice of anticoagulants by medical practitioners and patients using ISOP Israel new method.

Methods: A single medical center pilot project, governed by a multidisciplinary team, mentored by ISOP Israel, using validated tools for risk management and gaps analysis including 2017 ISMP Medication Safety Self-Assessment ${ }^{\circledR}$ for Antithrombotic Therapy and the Factor Mode and Effect Analysis method.

Results: The established multidisciplinary team has convened and reviewed the existing status of anticoagulants' practices in the pilot centre using the chosen tools. Opportunities for improvement were identified and prioritized. Multi-stage corrective and preventive action plan was set.

Initial interventions were implemented including:

Revised and updated protocols

- Reduction and restriction of anticoagulant inventory in the clinical wards

- Mandatory use of specific prescription forms for anticoagulants with special attention to the direct oral anticoagulants with systematic review by clinical pharmacists before delivery

- Improved availability of anticoagulant antidotes

- Establishing a direct access to the reimbursement process and authorisation of the Health Management Organisation to avoid treatment discontinuation

The next steps are focused on knowledge evaluation and education of both practitioners and patients through:

- Use of appropriate assessment questionnaires that will be led by the multidisciplinary team in all departments using anticoagulants

- Preparation and execution of an educational program with up-to-date information handouts and tutorials.

- Set up of a systematic review of electronic medical records data-base for the detection and analysis of anticoagulant related adverse reactions and medication errors.
- Putting forward recommendations for upgrading the computerized order entry system to the organization management.

Conclusion: The establishment of a dedicated multidisciplinary team with the mentoring of ISOP Israel professionals and the use of validated tools for risk assessment at the system organisation level facilitated the initial implementation of a risk minimization plan for anticoagulant medication use. Upon completing all planned stages, the expected benefits can be evaluated to justify regional and national expansion of the program and its methodology.

\section{Interaction between rosuvastatin and ticagrelor resulting in rhabdomyolysis}

\author{
V. Macolic Sarinic ${ }^{1}$, L. Sandberg ${ }^{2}$, J. Hartman ${ }^{2}$, P. Caduff- \\ Janosa $^{2}$ \\ ${ }^{1}$ Agency for Medicinal Products and Medical Devices, Zagreb, \\ Croatia, ${ }^{2}$ WHO Collaborating Centre for International Drug \\ Monitoring, Uppsala Monitoring Centre, Uppsala, Sweden
}

Background: A signal screening focusing on drug-drug interactions in VigiBase, the WHO global database of individual case safety reports, identified a case series pointing to an interaction between ticagrelor and rosuvastatin leading to rhabdomyolysis. Rhabdomyolysis is a well-known adverse drug reaction of statins. The risk of developing rhabdomyolysis is concentration dependent and is increased in elderly patients and in patients with renal and/or hepatic impairment [1].

Aim: To explore a possible interaction between rosuvastatin and ticagrelor leading to rhabdomyolysis.

Methods: Clinical review of reports with rhabdomyolysis and concurrent use of rosuvastatin and ticagrelor, included in VigiBase up to October 2016.

Results: VigiBase contained five unique cases reporting rhabdomyolysis with ticagrelor and rosuvastatin as suspected medications. The reports originated from Bulgaria, Canada, Greece, the Netherlands [2], and the United States. Four patients were males and one was probably erroneously coded as female. The patients were 70-82 years old except for a 46-yearold one. Times to onset of symptoms after start of rosuvastatin and ticagrelor were one month (three cases), 1.5 years (one) and unknown (one). In two cases the patient had used rosuvastatin for years without complaints before ticagrelor was added. The elderly patients received daily doses of $40 \mathrm{mg}$ rosuvastatin (two cases), $20 \mathrm{mg}$ (one) and an unknown dose (one); the younger patient received $20 \mathrm{mg}$ daily. Two cases reported concomitant use of ezetimibe, which increases the AUC of rosuvastatin 1.2 times [1]. One case reported use of lisinopril, which can cause renal dysfunction [3].After discontinuation of both ticagrelor and rosuvastatin in two cases, the symptoms regressed or disappeared.

Conclusion: Three possible interaction mechanisms can result in an increased concentration of rosuvastatin when used in combination with ticagrelor: (1) renal impairment caused by ticagrelor, (2) competition on transporter level as both drugs are P-gp substrates and ticagrelor is also a weak P-gp inhibitor, (3) P-gp and/or UGT2B7 genetic polymorphism [4,5]. The reports in VigiBase presented one or more risk factors for rhabdomyolysis: old age, higher than recommended rosuvastatin dose, and/or concurrent use of drugs that may affect rosuvastatin concentration. In these cases, the start of ticagrelor seems to have added an additional risk, raising rosuvastatin concentration to critical levels, resulting in rhabdomyolysis. This is supported by a plausible temporal association in three cases. In conclusion, the reports in VigiBase, together with plausible 
mechanisms, support a signal for an interaction between ticagrelor and rosuvastatin especially in high-risk patients.

Further sources of information/References

1. UK Medicines \& Healthcare products Regulatory Agency: Summary of Product Characteristics for rosuvastatin. Available from: http://www.mhra.gov.uk/home/groups/spcpil/documents/spcpil/ con1486707217805.pdf. Accessed: April 2017

2. van Vuren AJ, Jong B, Bootsma HPR, van der Veen MJ, Feith GW. Ticagrelor-induced renal failure leading to statin-induced rhabdomyolysis. Neth J Med 2015; (73):136-8

3. UK Medicines \& Healthcare products Regulatory Agency: Summary of Product Characteristics for lisinopril. Available from: http://www.mhra.gov.uk/home/groups/spcpil/documents/spcpil/ con1485495764996.pdf. Accessed: April 2017

4. European Medicines Agency: Summary of Product Characteristics for ticagrelor. Available from: http://www.ema.europa.eu/docs/ en_GB/document_library/EPAR_Product_Information/human/0012 41/ WC500100494.pdf. Accessed: April 2017.

5. Varenhost C, Eriksson N, Johansson A, Barratt BJ, Hagstrom E, Akerblom A, et al. Effect of genetic variations on ticagrelor plasma levels and clinical outcome. Eur Heart J 2015; (35):1901-12

\section{Analysis and Comparison of Risk Management Tools}

\author{
B. Unal ${ }^{1}$, S. Sardas ${ }^{1}$ \\ ${ }^{1}$ Marmara University, Faculty of Pharmacy, Toxicology Department, \\ Pharmacogenetics and Drug Safety Unit, Istanbul, Turkey
}

Background: All medicinal products are associated with some risks, and the broad purpose of Risk Management Plans (RMPs) is to ensure that these are actively managed in order, as far as possible, to minimise Serious Adverse Drug Reactions (SARs) in users. Over the recent years, some of the improvements of the PV system implemented in Turkey points out the fact that risk minimization tools are now an essential part of the safety management of medicines.

Objective: To analyse risk minimisation tools applied for drugs in Turkey, compare with EMA and FDA tools in order to have an enhanced overview.

Method: The risk management tools that are implemented in Turkey were analyzed according to the active substance, Anatomic Therapeutic Chemical (ATC) classification, safety concerns and compared with the Risk Minimisation Activities (RMAs) based on the European Public Assessment Reports (EPARs) of EMA and with the Risk Evaluation and Mitigation Strategies (REMS) of FDA. The advantages and disadvantages of the tools are the scope of this helicopter view study.

REMs and EPARs from respective websites and publication sources are used for a detailed review. The drugs with Turkish Drug and Medical Device Agency (TITCK) RMAs were identified and compared with both an approved REMS and an EPAR. The drugs for which only the distribution of DHCPL have been implemented are excluded.

Results: Monitoring the safety of drugs has been developed in Turkey recent 12 years and the legislation which is revised in 2014 triggered a milestone activity in pharmacovigilance system. The RMAs have been applied for either drugs approved or with experience in the market. It is identified that that most frequent RMA is the provision of educational material; antineoplastic and immunomodulating agents (17) most often had RMA which is similar to EMA and FDA. Out of these 37 drugs with RMAs only 11 of them have both additional RMAs in EPARs and REMS. There are approved REMS for 14 drugs with RMAs. On the other hand for 12 drugs with RMAs, it is observed that there are only RMAs in EPARs but not approved REMS.

Conclusion: Overall, this analysis shows that, although comprehensive measurement and evaluation are essential, there is no one single or ideal tool for communication about benefits and risk with patients/healthcare professionals. Therefore more efficient, independent, effective tools should be developed. New models and communication platforms, the use of information technology such as web-based systems is needed which provides transparency in communication and collaboration with health authorities.

Further sources of information/References:

1. Calvo B, Zuniga L (2011). Risk Management Plan and Pharmacovigilance System -Biopharmaceuticals: Biosimilars. In: Risk Management Trends. Ed: Nota G, Intech, Rijeka, Croatia.

2. Kasap Y, et al. The Drugs Monitored by TUFAM with Risk Management Plan (RMP). Drug Saf 2011; 34 : 906-7

3. RxMediaPharma ${ }^{\circledR} 2015$, Interactive Drug Information Resource

4. Unal B, et al. Risk Minimization Tools in Turkey Compared by EMA RMP and FDA REMS. Drug Saf 2012; 35: 920

5. PV legislation strengthens drug safety monitoring in EU. Reactions Weekly 2016; 1615:5

\section{Evaluation of a predictive model for suspected drug-drug interactions in routine signal detection}

S. Hult ${ }^{1}$, T. Bergvall ${ }^{1}$, T. Bradley ${ }^{2}$, L. Gattepaille ${ }^{1}$, B. Grundmark $^{1}$, D. Sartori ${ }^{1}$, S. Vidlin ${ }^{1}$, G.N. Norén ${ }^{1}$, J. Ellenius ${ }^{1,3}$

${ }^{1}$ Uppsala Monitoring Centre, WHO Collaborating Centre for International Drug Monitoring, Uppsala, Sweden, ${ }^{2}$ Karolinska Institutet, Division of Clinical Pharmacology, Stockholm, Sweden, ${ }^{3}$ Karolinska Institutet, Department of Learning, Informatics, Management and Ethics, Stockholm, Sweden

Background: Approximately 17\% of adverse drug reactions (ADRs) in hospital admissions are caused by interactions [1].Reports in VigiBase, the WHO global database of individual case safety reports, often concern multi-drug users at risk of drug-drug interactions, and should be valuable for finding interaction signals. A dedicated signal detection algorithm, vigiRank for Interactions, has been developed at Uppsala Monitoring Centre (UMC) [2].

Aim: To apply and evaluate vigiRank for Interactions in routine signal detection at UMC

Methods: Drug-drug-ADR combinations were retrieved from VigiBase after removal of suspected duplicate reports identified by vigiMatch [3].Combinations with more than 30 reports, only one country, no new reports in two years and non-serious events, and historically checked combinations were excluded. Remaining combinations were assigned vigiRank scores between 0 and 1.00, where higher values indicate higher likelihood for an interaction signal. Combinations were assessed manually in vigiRank order with the opportunity to include other reactions for the same drug-drug pair even though they may have lower vigiRank scores. A Microsoft Access interface was developed to facilitate assessment of combinations, and provide fields for documentation. Labelling status of drug-drug-ADR interactions was controlled in relevant sources $[4,5]$.Stockley's Drug Interactions and Janusmed ${ }^{[6]}$ were integrated in the interface to automatically exclude known interactions. Combinations 
classified as potential signals were assessed in-depth by members of the UMC expert review panel.

Results: Seventy-five of 668 assessed drug-drug-ADR combinations were selected for further review, ultimately leading to eight potential signals, where some included several similar combinations. Another eight combinations were decided to be kept under review, and 585 were dismissed. Of the latter, 246 were non-suggestive of an interaction and 209 concerned known interactions. Among the potential signals 75\% (6/8) had vigiRank scores in the range 0.90 to 1.00 compared to $33 \%$ (219/668) among all assessed combinations. All potential signals concerned only drugs with a first report in VigiBase year 2003 or earlier. Of the six potential signals assessed in-depth so far, two have been published in the UMC SIGNAL document.

Conclusion: Signals of drug-drug-ADR interactions can be identified in VigiBase using a predictive algorithm to direct clinical review. There were no new drugs among the detected potential signals. Examples of obstacles were lack of sufficient information on many reports, and remaining duplicates. Effectiveness of exclusion criteria will be further evaluated in future UMC signal screenings.

Further sources of information/References

1. Pirmohamed M, James S, Meakin S, Green C, Scott AK, Walley TJ, et al. Adverse drug reactions as cause of admission to hospital: prospective analysis of 18820 patients. BMJ 2004;329:15-9

2. Strandell J, Caster O, Hopstadius J, Edwards IR, Norén GN. The development and evaluation of triage algorithms for early discovery of adverse drug interactions. Drug Saf 2013;36:371-88

3. Norén GN, Orre R, Bate A, Edwards IR. Duplicate detection in adverse drug reaction surveillance. Data Min Knowl Discov 2007; $14: 305-28$

4. Electronic Medicines Compendium. Available from: http://www.medicines.org.uk/emc. Accessed: September 2016

4. DailyMed. Available from: https://dailymed.nlm.nih.gov/dailymed. Accessed: September 2016

4. Janusmed. Available from: https://janusmed.sll.se. Accessed: September 2016

\section{A Comparison of Classification Procedures and Custom Event Terms in Differentiating Drug Scheduling Classes}

\section{Hauben ${ }^{1,2}$, E. Hung ${ }^{1}$}

${ }^{1}$ Pfizer Inc, New York, USA, ${ }^{2}$ New York University Medical Center, New York, USA

Introduction: Abuse liability potential is a pharmacovigilance $(\mathrm{PhV})$ specialty that is arguably underrepresented in the $\mathrm{PhV}$ literature. This is in part because pre-market evaluation includes a multi-component abuse liability assessment that mitigates the possibility of post-market drug rescheduling. Nonetheless, there have been instances of signals detected in the postmarketing period related to possible rescheduling, but there is limited systematic study of the utility of quantitative analysis of spontaneous reporting system (SRS) data for detecting and assessing these signals and supporting rescheduling deliberations. If drug scheduling assignments map to discriminating patterns of spontaneous reported adverse drug events, multivariate analysis of SRS data may play a supportive role.

The terminological representation of abuse-related events can be quite expansive, including terms of varying specificity. The safety analyst has various options for defining health outcomes of interest including individual
Preferred Terms (PTs), Standardized MedDRA Queries (SMQs) and userdefined custom terms. The motivation of many predefined set of PTs, such as SMQs, is case finding rather than aggregate quantitative analysis, so there is a limited experience applying multivariate statistics in this domain [1-4]. Objective: To compare how well three different abuse-related event sets discriminate scheduling classes in multivariate discriminant analysis (MDA) of spontaneous reports.

Methodology: The components of our methodology are: 1) Three sets of abuse-related events: narrow SMQ PTs, broad SMQ PTs, and custom event group terms by Love and Sun (L\&S) [5]. 2) A test set of reference drugs representing the various US scheduling classes (CS I-V). 3) Disproportionality metrics for the corresponding drug-event pairs. 4) MDA, including step-wise MDA were applied to untransformed data, logged data, and principle components (PC).

Results: In MDA the maximum achievable classification performance was less than $65 \%$. For the L\&S custom terms, stepwise MDA selected drug abuse and vocal as the individual event terms best able to differentiate scheduling classes. For the MedDRA SMQ, Substance abuse PT and Drug diversion PT were the individual terms with the most discriminative power in untransformed data. Log transformation and analyzing PCs did not materially improve or degraded performance.

Discussion: MDA was not effective in discriminating drugs by scheduling class. We consider the unsatisfactory performance in light of the adequacy of the data related to sample size, statistical assumptions of MDA, dimensionality, multicollinearity and the differing objectives of classification and PC analysis.

Further sources of information/References

1. Arons C, Hung E, Ratcliffe S, Feltner D, Hauben M. Can data mining spontaneously reported adverse events be used to assess drug abuse potential. PharmacoepidemiolDrug Saf 2010;19:S302-S3

2. Hauben M, Arons C, Hung E, Ratcliffe S. Application of multivariate statistical methods to the assessment of drug abuse potential based on spontaneously reported adverse events. PharmacoepidemiolDrug Saf 2014;23:145

3. Arons C, Hung E, Ratcliffe S, Hauben M. Using data mining of spontaneous adverse event reports and multivariate statistical methods for assessing abuse potential. Drug Alcohol Depend 2015;146:e210.

4. Hauben M. Multivariate analysis for visualizing pharmacovigilance data. Drug Saf. 2015;38:956-7

5. Love LA, Sun S. Proposed Query to Capture Abuse-Related Adverse Events. College on Problems of Drug Dependence-75th Annual Meeting, San Diego, CA, June 2013.

\section{Fatal toxicity with a single IM dose of methotrexate in a patient treated for an ectopic pregnancy}

\author{
M. Bouhlel ${ }^{1}$, R. Charfi $^{1}$ \\ ${ }^{1}$ National Center of Pharmacovigilance, Tunis, Tunisia
}

Background: The systemic injections of methotrexate (MTX) have emerged as an alternative management for women with ectopic pregnancy. It was then documented that ectopic pregnancy was successfully treated with a single intra muscular (IM) injection of MTX [1].

The MTX, an antifolate acting by inhibition of DHF, an enzyme crucial in the metabolism of folic acid, has many side effects that can be observed at high and low doses such as :skin, gastrointestinal, renal, hepatic and hematologic toxicity [2]. 
Objective: We report a case with a rare toxicity of single IM MTX injection $(2500 \mathrm{mg}$ ) where the issue was fatal.

Case report: A 37-year-old woman, with a history of arterial hypertension, of penicillin allergy and of a primary sterility treated with ovulation inductor. She suffers also with a sleep apnea syndrome.

She received in one dose $25 \mathrm{mg} / \mathrm{kg}$ of MTX IM to evacuate an ectopic pregnancy diagnosed by $30,000 \mathrm{UI} / \mathrm{l}$ beta hCG level. The first two days after, she developed skin lesions: erythema and a rash. She received three injections of corticosteroid therapy in three days. Forty-eight-hours after, the skin lesions got worse associating edema, gingival lesion, accelerating of sedimentation speed and the patient became agitated. A week later, the patient was hospitalized because of a bone marrow aplasia, hepatic cytolysis (4 times upper limit), renal failure (creatinine $370 \mu \mathrm{mol} / \mathrm{L}$ ) and disseminated intravascular coagulation. She also presented a digestive toxicity associating melena, hematemesis, hemorrhage and vomiting. In addition, our case developed mucositis and bronchial congestion leading to respiratory failure. The plasma level of MTX at hospitalization (day 10) was $0.179 \mu \mathrm{M}$. We suspected a MTX intoxication. She was treated by folinic acid, and an empirical antibiotic therapy. She was also transfused by packed of red bloods cells. At day 11, she died after a severe septic shock.

Discussion/conclusion: Success rates of single-dose MTX in the treatment of ectopic pregnancy have been reported to be as high as $94 \%$, with lower toxic side effects, fewer complications and better patient acceptance as compared to multi-dose regimens [3].

In our case, we report a many of side effects of MTX with a high single dose $(2500 \mathrm{mg}) \mathrm{IM}$. The issue was fatal explained with the MTX mechanism of acting. By affecting replication of bone marrow cells, MTX induce thrombocytopenia, lymphopenia and granulocytopenia leading to life-threatening infections.

However, with single dose form of MTX, very low incidence of side effects was reported as mentioned [4].

The first case of life-threatening neutropenia following single dose of MTX $(1 \mathrm{mg} / \mathrm{kg})$ to treat ectopic pregnancy was reported in 1996 [5]. Also we had a case in our center on 2013 of MTX toxicity with a lower doses and blood concentration on day $11(0,05 \mu \mathrm{M})[6]$.

This case has shown that the toxicity of MTX can be observed with a single high dose.

\section{Further sources of information/References :}

1. Mirbolouk F, et al. Predicting factors of medical treatment success with single dose methotrexate in tubal ectopic pregnancy: a retrospective study. Iran J Reprod Med 2015; 13: 351-4

2. Gaies E, Jebabli N, Trabelsi S, Salouage I, Charfi R, et al. Methotrexate Side Effects: Review Article. J Drug Metab Toxicol 2012; 3:125

3. Stovall TG, Ling FW. Single-dose methotrexate: an expanded clinical trial. Am J Obstet Gynecol 1994; 170:1840-1

4. The Practice Committee of the American Society for Reproductive Medicine. Medical treatment of ectopic pregnancy: a committee opinion. Fertil Steril 2013;100:638-44

5. Isaacs JD Jr, McGehee RP, Cowan BD. Life-threatening neutropenia following methotrexate treatment of ectopic pregnancy: a report of two cases. Obstet Gynecol 1996; 88(4 Pt 2):694-6

6. Gaies E, Ben Sassi M, Charfi R, Lakhal M, Klouz A, Trabelsi $\mathrm{S}$, Salouage I. Fatal methotrexate toxicity in a patient treated for an ectopic preganancy. Therapie 2016;71:348-50
215 Adverse events following immunization of pregnant women with Yellow Fever Vaccine in Kinshasa

D. Nzolo Bomene $\mathbf{e}^{\mathbf{1 , 2 , 3}}$, A. Engo Biongo ${ }^{1,3}$, T. Mpiempe Ngamasata $^{1,3}$, G. Mesia Kahungu ${ }^{1,3}$, S. Mampunza ${ }^{1,3}$, G. Tona Lutete $^{1,3}$, J.P. Van geertruyden ${ }^{2}$

${ }^{1}$ Unit Of Clinical Pharmacology and Pharmacovigilance, Kinshasa, Democratic Republic of the Congo, ${ }^{2}$ University of Antwerp, Antwerp, Belgium, ${ }^{3}$ National Pharmacovigilance Center, Kinshasa, Democratic Republic of the Congo

Introduction: Pregnancy is characterized by Immunological and physiological changes that may alter the susceptibility of mothers and foetuses to infectious diseases, as well as the immune response, resulting in serious outcomes. There is a concern whether or not one should vaccinate pregnant women with currently available vaccines. Unknown safety profile of live attenuated vaccines makes them contraindicated in pregnancy. In August 2016, following an outbreak of yellow fever vaccine, all the population were vaccinated in Kinshasa, DR Congo. It was recommended to administer normal dose $(0.5 \mathrm{ml})$ of yellow fever vaccine to all the pregnant women.

Aim: To determine most reported adverse event following vaccination of pregnant women.

Methods: A cross-sectional investigation was carried out in health facilities after vaccination campaigns. Pregnant women attending antenatal care at the moment of the investigation were interviewed by trained students from the Protestant University of Congo about their acceptance of mass vaccination. Experience about adverse events were requested from those who were vaccinated. Vigiflow software was used for adverse events data entry. Stata 12 was used for data analysis.

Results: A total of 333 pregnant women from 14 health facilities were interviewed. The mean age was 27.9 -year (95\% CI 27.1-28.7). The median gravidity was 3 (IQR: 1 ; 4). Of the 333 pregnant women interviewed, 279 accepted vaccination (83.8\%; 95\% CI 79.8-87.8). Out of 277 vaccinated pregnant women, $62(22.4 \%$; 95\% CI 17.4-27.3) reported 100 adverse events (average 1.6 AEFI per subject). Among vaccinated subjects (277), the most commonly reported SOCs were body as a whole—general disorders $(12.6 \%)$, Neurological disorders $(7.6 \%)$ and Gastrointestinal disorders $(6.8 \%)$. The most frequently reported PTs were asthenia $(6.5 \%)$, dizziness $(6.5 \%)$ and fever (3.6\%) While the most reported local reactions were Injection site pain $(1.1 \%)$, pruritus $(0.7 \%)$ and reaction $(0.7 \%)$. Adverse events of special interest included cases of uterine contraction and vaginal hemorrhage following immunization.

Conclusion: The safety profile of yellow fever vaccine in pregnant women appears to be acceptable. However, these results should be interpreted with caution since serious adverse events like abortion could not be identified in a cross-sectional investigation during antenatal care. A follow up of vaccinees until delivery could provide a better picture of adverse pregnancy outcomes for foetuses exposed to yellow fever vaccine during pregnancy. 


\section{Primary challenges to conducting post-marketing observational research in Sub-Saharan Africa: preliminary results}

\section{DeVore ${ }^{1,2}$}

\section{${ }^{1}$ University of Portsmouth, Portsmouth, UK, ${ }^{2}$ Drug Safety Research Unit, Southampton, UK}

Introduction: Due to efforts of many private and publicly funded health initiatives, drugs and vaccines are now reaching unprecedented numbers of people in resource-poor regions of Sub-Saharan Africa, a region with 70 percent of the population of one billion living under the poverty line [1]. Despite comprehensive and stringent phases of clinical trials, adverse drug reactions repeatedly occur after a drug is marketed, making post-marketing observational research vital to effective pharmacovigilance efforts. However, while nowhere is the need for a more efficient and more effective pharmacovigilance system as strong as it is in Sub-Saharan Africa, observational research efforts there, meant to bolster pharmacovigilance systems, are met with a host of external barriers, unique to, or exacerbated by the lack of resources.

Aim: To identify and quantify the degree that external factors, associated with resource-poor regions, affect the ability to successfully conduct postmarketing observational research in Sub-Saharan Africa.

Methods: A questionnaire-based survey was completed by 102 authors and co-authors of published literature pertaining to post-marketing observational studies with a Sub-Saharan African component, which had been published from 2013-2016. The respondents were asked to rank given, potential barriers to their research on a Likert Scale of $1-5$, from no impact to high impact. The Likert Scale data were given qualitative utility values and treated as interval data, and their weighted mean $\left(_{w}\right)$ calculated and ranked. Results: The majority of the survey respondents $(58.5 \%, \mathrm{n}=102)$ were medical doctors, prospective cohort studies (including CEM) were the most common type of post-marketing observational research conducted $(59.6 \%, \mathrm{n}=141)$, and district hospitals were the most common research setting, $(74.8 \%, \mathrm{n}=123)$. 'Limited technological infrastructure' was the highest ranked barrier to research $\left({ }_{w}=3.12\right)$, followed by 'Insufficient laboratory facilities' $\left({ }_{w}=3.07\right)$ and 'Insufficient local medical staff' $\left({ }_{w}=3.01\right)$. Cumulatively, the barriers had the greatest impact on laboratory staff $\left({ }_{w}=3.19\right)$, followed by statisticians/data analysts $\left({ }_{w}=3.10\right)$. Nurses and medical students reported the least degree of impact from the given barriers, ${ }_{w}=2.19$ and ${ }_{w}=1.91$, respectively.

Conclusions: The weighted mean scores of the highest ranked nine barriers to observational research were fairly tightly grouped around the score of 3 (moderate impact), however there was a greater degree of spread amongst the various occupational groupings.

Further sources of information/References:

1. The World Bank. Poverty headcount ratio. [website] 2017, Available from: http://data.worldbank.org/topic/poverty?locations=ZG [accessed 27-Feb-2017]

\begin{tabular}{|l|l|}
\hline $\begin{array}{l}\text { Barrier to conducting observational research in Sub-Saharan Africa ranked, } \\
\text { most to least impactful }\end{array}$ & $\begin{array}{l}\text { Weighted } \\
\text { Average }\end{array}$ \\
\hline Limited technological infrastructure (electricity, internet, telephone) & 3.12 \\
\hline Insufficient laboratory facilities & 3.07 \\
\hline Insufficient local medical staff & 3.01 \\
\hline Local medical staff not accustomed to research practices & 3.00 \\
\hline Local medical staff not trained in ADR detection or reporting & 2.95 \\
\hline High number of participants lost to follow-up & 2.95 \\
\hline Insufficient resources for study monitoring & 2.90 \\
\hline Higher cost to sponsor of conducting research in a resource-limited location & 2.89 \\
\hline Remote location (physical geography, transportation, infrastructure) & 2.86 \\
\hline
\end{tabular}

218 Making safer medicines: improving labeling and packaging to prevent medication errors

\author{
$\underline{\text { S. Garnica }^{1}}$, A. Aristizabal ${ }^{1}$ \\ ${ }^{1}$ Vitalis, Bogota, Colombia
}

Introduction: Labeling and packaging problems have been cited as the cause of $33 \%$ of medication errors (ME), including 30\% of related fatalities [1]. These same issues were identified as the second cause of ME in hospitals [2]. In Colombia, several medicines at hospital settings are prone to ME because their similarity and look-alike names, needing interventions to reduce this problem [3]. Almost one in every three injectable medicines used at Colombian hospitals has Vitalis as Medication Authorization Holder [4]. Unpublished data from some health companies in Colombia showed that $255 \mathrm{ME}$ were caused by similarity and names of Vitalis medicines in 2016.

Aim: To improve labeling, packaging and naming of medicines to prevent ME. Methods: We performed a risk analysis of Vitalis ampoule medicines prone to $\mathrm{ME}$, following international guidance for safe medication design, to finally determine specific problems and improve labeling, packaging and naming of products at risk.

Results: 40 medicines were included, representing 62 million units manufactured in 2016. We classified them by ampoule size, clear or amber glass, and label text color. 18 products $(45 \%)$ were identified as high risk because their similarity. The main factor led on similarity was low background contrast and use of clear labels. Other related factors for ME included low readability and visibility, cluttered labeling, unclear strength designations, and look-alike names [1]. We designed new labels with white label background, color differentiation between similar products and strength designations, vertical text orientation for complete reading, and Tall-man lettering. Carton labeling also was improved by addition of essential information on the main package faces [5].

Conclusions: Safety by design allow manufacturers mitigate risks of ME following international guidance. Further research is needed to measure impact of this intervention.

\section{Further sources of information/References}

1. Committee on Identifying and Preventing Medication Errors., Aspden P, Wolcott J. Preventing Medication Errors. Institute of Medicine. 1st ed. National Academies Press; 2007

2. Otero M, Codina C. Errores de medicación: estandarización de la terminología y clasificación. Farm Hosp 2003;27:137-49

3. Machado J, Moreno P. Hospital medication errors in a pharmacovigilance system in Colombia. Farm Hosp 2015;39:338-49

4. Sistema Información Precios Medicamentos-SISMED [Internet]. 2017 [cited 2 May 2017]. Available from: http://www.sispro.gov.co/ recursosapp/app/Pages/SISMED.aspxIMSN

5. Position Statement: Making Medicines Naming, Labelling and Packaging Safer. ISMN-International Medication Safety Network. 2013. [Internet]. Available from: www.ismp.org

6. FDA and ISMP Lists of Look-Alike Drug Names with Recommended Tall Man Letters. ISMP 2011. [Internet] Available from: https:// www.ismp.org/Tools/tallmanletters.pdf 


\section{Methadone side effects and intoxication}

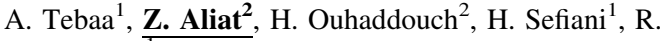 \\ Soulaymani \\ ${ }^{I}$ Centre Anti Poison et de Pharmacovigilance, Rabat, Morocco, \\ ${ }^{2}$ Faculty of Medicine and Pharmacy, Rabat, Morocco
}

Introduction: The Methadone is indicated as a replacement therapy for major opiate drug dependence. Its prescription aims to decrease the compulsion of search for morphine and to calm the withdrawal syndrome [1-4]. However, it has side effects that often have an impact on the quality of life of patients and the risk of poisoning, which may be deliberate or accidental.

AIM: To highlight the most reported side effects on patients on methadone in addition to the incidence of intoxications and their occurrence circumstances.

Methods: This is a retrospective analysis of the database of the Centre Anti Poison et de Pharmacovigilance du Maroc concerning the methadone, over a period of 2 years, since 2015 .

Results: Our analysis of the database of the CAPM showed 12 reported cases. $28.6 \%$ of the side effects were gastrointestinal effects (constipation and vomiting), the same rate was observed for reproductive disorders (erectile dysfunction and gynecomastia). Skin and appendage disorders were observed in $21.4 \%$ of cases. Six cases of methadone intoxication were reported, from which 4 were accidental. The observed symptoms were: obliteration, asthenia, sweating, loss of consciousness, vomiting, redness, myosis. A symptomatic treatment was recommended to 4 patients.

Conclusion: This study allowed us to identify the most common side effects noticed in patients on methadone, and to demonstrate the underreporting in the pharmacovigilance by the low rate of reported cases, hence the importance of developing a positive attitude towards pharmacovigilance among health professionals in such a way that reporting becomes an accepted and assimilated routine behavior. It is also important to put in place a good therapeutic education of methadone patients in order to reduce cases of drug intoxication.

Further sources of information/References :

1. Laqueille X, Vazquez V. Aspects pharmacologiques et neurobiologiques de la méthadone dans le traitement des hérö̈nomanies. Ann Med Psychol (Paris) 2004;162:302-6

2. Lauzon P. Les effets indésirables des médicaments de substitution dans le traitement de la dépendance aux opioïdes. Drogues, santé et société $201110: 51-91$

3. Théberge A, Néron A.. L'implantation d'un nouveau protocole sur la méthadone. Pharmactuel 2006;39: 218-24

4. Paquin, I. Le traitement de substitution avec méthadone au Québec: une mesure de santé publique en réadaptation. Drogues, santé et société $2003 ; 2: 1-10$

\section{Hospital admission due to dysglycaemia among children in England and Wales}

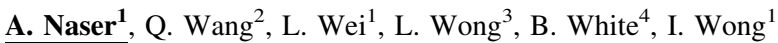 \\ ${ }^{1}$ Research Department of Practice and Policy-UCL School Of \\ Pharmacy, London, UK, ${ }^{2}$ Beijing Tongren Eye Center, Beijing \\ Tongren Hospital, Capital Medical University, Beijing, China, ${ }^{3} U C L$
}

Institute of Child Health, London, UK, ${ }^{4}$ Child and Adolescent Diabetes Service, UCL Hospitals NHS Foundation Trust, London, UK

Introduction: Hypoglycaemia and hyperglycaemia are considered to be serious and potentially life threatening adverse events associated with patients' inappropriate use of anti-diabetic medications [1].

A previous study from the USA showed that hypoglycaemia increased by $11.7 \%$ and hyperglycaemia declined by $38.6 \%$ in the elderly patients between 1999 and 2011 [2]. In England, a recent study showed that hypoglycaemic admissions increased by $14 \%$ between 2005 and 2014 [3]. It is not clear whether hyperglycaemia in children have similar trends. In order to address this question, we conducted an observational study to explore national trends in hospital admissions due to dysglycaemia in children (aged 14 years old and under) in England and Wales from 1999 to 2015.

Aim: To investigate trends in paediatric hospital admissions due to hypoglycaemia and hyperglycaemia in England and Wales.

Methods: We performed a secular trend study using publicly available national data: Hospital Episode Statistics (HES) in England [4] and Patient Episode Database for Wales (PEDW) (April 1999-March 2015) [5]. The HES and PEDW contains hospital admission data for hypoglycaemia and hyperglycaemia in children (aged 14 years and under). Hospital admissions were identified using the diagnostic codes of E16.0, E16.1, E16.2 and T38.3 for hypoglycaemia admissions, and E10.1, E11.1, E12.1, E13.1, E14.1, and R73.9 for hyperglycaemia admissions from the $10^{\text {th }}$ version of the International Statistical Classification of Diseases and Related Health Problems. Mid-year population data (1999-2015) were collected from the Office for National Statistics (ONS) [6] _ENREF_2. Hypoglycaemia and hyperglycaemia admission trends over the study period were assessed using a Poisson model.

Results: In 1999 there were 1228 hospital admissions for hypoglycaemia and 2721 in 2015. Between 1999 and 2015, the hospital admission rate for hypoglycaemia increased by $112.9 \%$ (from 12.39 [95\% CL, 11.70-13.09) to 26.38 [(95\% CI, 25.39-27.38) per 100,000 persons]. However, there were 1684 hospital admissions for hyperglycaemia in 1999 and 2292 in 2015. The hospital admission rate for hyperglycaemia increased by $30.8 \%$ (from 17.00 [95\% CI, 16.18-17.81) to 22.22 [95\% CI, 21.31-23.13) per 100,000 persons) for the same period.

Conclusions: This is the first time the rate of hospital admission due to hypoglycaemia overtook hyperglycaemia in recent years. Further research is urgently needed to identify the causes of increased hospital admission due to hypoglycaemia and hyperglycaemia in children so that appropriate interventions can be developed to reduce this life threatening adverse event.

\section{Further sources of information/References}

1. Umpierrez G, Korytkowski M. Diabetic emergencies-ketoacidosis, hyperglycaemic hyperosmolar state and hypoglycaemia. Nat Rev Endocrinol 2016;12:222-32

2. Lipska KJ, Ross JS, Wang Y, et al. National trends in US hospital admissions for hyperglycemia and hypoglycemia among Medicare beneficiaries, 1999 to 2011. JAMA Intern Med 2014;174:1116-24

3. Zaccardi F, Davies MJ, Dhalwani NN, et al. Trends in hospital admissions for hypoglycaemia in England: a retrospective, observational study. Lancet Diabetes Endocrinol 2016;4:677-85

4. Health and Social Care Information Centre (HSCIC). Hospital Episode Statisitcs. 2017; http://content.digital.nhs.uk/searchcatalo gue?q=title $\% 3 \mathrm{~A} \% 22 \mathrm{Hospital}+$ Episode+Statistics\%2C+Admitted+ patient+care+-+England $\% 22 \&$ area $=\&$ size $=10 \&$ sort=Relevance. Accessed February 01, 2017

5. NHS Wales Informatics Service. Annual PEDW Data Tables. 2017; http://www.infoandstats. wales.nhs.uk/page.cfm?pid=41010\&orgid= 869. Accessed February 01, 2017 
6. Office for National Statistics (ONS). Population estimates. https://www.ons.gov.uk/peoplepopulationandcommunity/population andmigration/populationestimates/datasets/populationestimates forukenglandandwalesscotlandandnorthernireland. Accessed February 01,2017

\section{Contributing factors to the appearance of adverses events related to injectable Iron complexes}

\author{
A. Tebaa ${ }^{1}$, H. Ouhaddouch ${ }^{2}$, Z. Aliat ${ }^{2}$, G. Benabdellah ${ }^{1}$, \\ L. Aitmoussa ${ }^{1}$, R. Soulaymani ${ }^{1}$ \\ ${ }^{1}$ Centre Anti Poison et de Pharmacovigilance du Maroc, Rabat, \\ Morocco, ${ }^{2}$ Faculty of Medicine and Pharmacy of Rabat, Rabat, \\ Morocco
}

Introduction: In solution, the iron is associated with molecules of water to form molecules of ferric hydroxide. The latter are a very reactive entities which present themselves in iron solutions injectable in the form of complexes associated with molecules of carbohydrates whose nature varies from one specialty to another.

Aim: to review the different administration practices associated with the onset of allergic reactions of injectable iron and to give an overview of the recommendations proposed by the Centre Anti Poison et de Pharmacovigilance du Maroc (CAPM) in order to minimize these risks.

Methods: We conducted a retrospective analysis of adverse events cases spontaneously reported in the national database of CAPM since January 2015. A bibliographic search on the various search engines on the contributing factors to the occurrence of these events was achieved simultaneously.

Results: The study included 11 cases that all presented allergic hypersensitivity reactions of which 6 cases of anaphylactic shock. $45 \%$ of adverse events occurred under ferric hydroxide sucrose and 55\% under dextran ferric hydroxide.

The analysis and investigation showed that the main contributing factors identified were the non compliance to the route of administration as well as the volume of dilution and the appropriate duration of administration for each drug are responsible for to the occurrence of these allergic reactions [2]. In order to reduce the occurrence of these preventable anaphylactic reactions, the CAPM proposed a list of recommendations aimed at raising the awareness and information of health professionals about the modalities of prescription, preparation and administration of injectable iron.

Conclusion: The seriousness of injectable iron adverse events must raise awareness of the importance of the precautions for use of these complexes and of their modes of administration.

Further sources of information/References:

2. VL, Vacher. Complexes injectables de Fer : Différences au sein d'une même famille, Lettre ICAR en nephrol.2011

3. Iron Sucrose Injection. Official monograph in: The United States Pharmacopoeia, 2008

\section{Abnormalities in liver or kidney function tests among patients treated for multi-drug resistant Tuberculosis in Kinshasa}

Y. Lula ${ }^{1,2}$, A. Engo ${ }^{1}$, G. Nsimba ${ }^{1}$, J. Mbo ${ }^{4}$, R. Mulongo ${ }^{4}$, M. Kaswa $^{3}$, G. Mesia ${ }^{1}$, G. Tona ${ }^{1}$
${ }^{1}$ University of Kinshasa, Kinshasa, Democratic Republic of the Congo, ${ }^{2}$ University of Antwerp, Antwerp, Belgium, ${ }^{3}$ National Tuberculosis Programme, Kinshasa, Democratic Republic of the Congo), ${ }^{4}$ Management Sciences for Health, Kinshasa, Democratic Republic of the Congo

Background: Multi-drug resistant Tuberculosis (MDR TB) treatment is known to be associated with significative adverse events (AEs). Liver, renal or electrolytes disturbances such as hypokalemia are accounted among the most serious AEs which may lead to death or treatment interruption or modification. ${ }^{(1-3)}$ In the Democratic Republic of Congo (DRC), National TB programme recommends two regimens. The shorter regimen (SR) lasts 9 months and the second one is the 20-months course treatment.

Objective: The aim of this study was to describe and assess liver, renal enzymes or hypokalemia in MDR TB patients under treatment.

Method: This prospective cohort over one-year period (2015-2016) was carried out in 16 health centres dedicated to MDR TB management in Kinshasa (the capital city of DRC). Common terminology criteria for adverse events of US National cancer institute (version 4.03) applied to define grade of severity of laboratory abnormalities.

Results: Of 258 patients followed up, forty five (17.4\%) develop either liver or renal dysfunction or hypokalemia with a total of 56 laboratory abnormalities. Males accounted for up to $95 \%$ of patients, mean age was about 35 years. Almost patients $(95.6 \%)$ were under SR. Six patients were HIV infected and received concomitantly antiretroviral therapy. Liver abnormalities consisted of 13 cases (28.8\%) of mild elevated level of alanine aminotransferase (ALT) and aspartate aminotransferase (AST). Four cases $(8.9 \%)$ of more than threefold serum ALT or AST elevation were observed of which one patient with more than twentyfold ALT elevation. Eight patients $(17.8 \%)$ developed moderate to severe elevation of serum creatinine. And 7 patients (15.6\%) were diagnosed with severe hypokalemia.

Conclusions: Laboratory monitoring during the management of MDR TB is required especially as some abnormalities may be life threatening.

Further sources of information/References

1. Saukkonen JJ, Cohn DL, Jasmer RM, et al. An Official ATS Statement: Hepatotoxicity of Antituberculosis Therapy. Am J Respir Crit Care Med 2006; 174:935-52

2. Shin S, Furin J, Alcantara F, et al. Hypokalemia Among Patients Receiving Treatment for Multidrug-Resistant Tuberculosis. Chest 2004; 125:974-80

3. Chang $\mathrm{C}-\mathrm{H}$, Chen $\mathrm{Y}-\mathrm{F}, \mathrm{Wu} \mathrm{V}-\mathrm{C}$, et al. Acute kidney injury due to anti-tuberculosis drugs: a five-year experience in an aging population. BMC Infect Dis 2014; 14:23

\section{Use of Hospital Data to Estimate Burden of Adverse Drug Events}

\author{
$\underline{\text { M. Lillie }}{ }^{1}$, L. Sharma ${ }^{1}$ \\ ${ }^{1}$ Cognizant, London, $U K$
}

Introduction: Recently published data from the US's National Electronic Injury Surveillance System indicates four in a thousand individuals will attend an emergency department each year for a suspected adverse drug event (ADE) [1]. For countries without similar active surveillance systems, the availability of large hospital administrative or claims databases may provide an inexpensive method to estimate the burden and trends in hospital attendance for ADEs. 
Aim: Review clinical literature to compare the methodologies used to measure ADEs among hospital attending patients and to review burden of ADEs in these patient populations.

Methods: A preliminary search of Embase and PubMed databases identified 5285 and 4857 publications, respectively. Application of inclusion criteria: (1) publications with available abstracts, and (2) data repository covering at least one major urban area; and exclusion criteria: (1) publication focused on a single disease or medication category, or (2) data collected as part of study, only, and not part of a long-term routine data collection, resulted in final total of 31 relevant articles.

Results: A total of 20 different databases were identified in 31 publications (including four conference abstracts) covering eleven countries. A single database was identified in each of seven countries, while two or more different databases were identified in each of Australia, Brazil, Canada, and USA. Overall, data were collected from between 1981 to 2015 with most publications (19) reporting ADEs in the general population, seven focusing on elderly population and one publication investigating the hospital attendances of the pediatric population. The lowest reported proportion of ADEs (as a percentage of hospital admissions) was from Brazil's State of Rio de Janeiro (0.18\%) for 1998-2002 and the highest proportion (5.6\%) was reported from the USA's National Inpatient Sample database in 2006 [2,3]. The lowest rate of ADE hospital admissions per 1000 population was 0.01 among Brazil's elderly population (60+ years) while the highest rate (15.2) was reported among the elderly (60+ years) of Canada's province of Newfoundland [4,5].

Conclusions: Heterogeneity in healthcare attendance definitions, coding methodology, and ADE definitions makes international comparisons difficult. Further research is required to assess sensitivity and specificity of ADE coding.

Further sources of information/References

1. Shehab N, Lovegrove MC, Geller AI, Rose KO, Weidle NJ, Budnitz DS. US Emergency Department Visits for Outpatient Adverse Drug Events, 2013-2014. JAMA 2016; 316: 2115-25

2. Rozenfeld S. Drug adverse events in hospitals in the State of Rio de Janeiro, Brazil. Rev Saude Publica 2007; 41: 108-15

3. Stausberg J. International prevalence of adverse drug events in hospitals: an analysis of routine data from England, Germany, and the USA. BMC Health Serv Res 2014; 14: 125

4. de Paula TC, Bochner R, Montilla DE. Clinical and epidemiological analysis of hospitalizations of elderly due to poisoning and adverse effects of medications, Brazil from 2004 to 2008. Rev Bras Epidemiol 2012; 15: 828-44

5. Sikdar KC, Dowden J, Alaghehbandan R, MacDonald D, Peter P, Gadag V. Adverse drug reactions in elderly hospitalized patients: a 12-year population-based retrospective cohort study. Ann Pharmacother 2012; 46: 960-71

\section{Regulation of e-cigarettes: ensuring patient safety and product quality}

H. $\operatorname{Bird}^{1}$

${ }^{1}$ Medicines and Healthcare Products Regulatory Agency, London, UK

Background: The role of pharmacovigilance is expanding beyond traditional medicines to include previously unregulated products such as nicotine-containing e- cigarettes. Since the EU Tobacco Products Directive [1] came into force, the MHRA is responsible for safety regulation of these products in the UK . Although considered by Public Health England to be $95 \%$ safer than smoking tobacco cigarettes [2], it cannot be assumed that these products are entirely risk free.

Objective/Aim: The Yellow Card reporting scheme has been expanded to allow reporting of suspected adverse reactions and safety concerns regarding nicotine-containing e-cigarettes and refills from healthcare professionals and members of the public. Currently minimal safety information about these products is known; the aim of extending the reporting scheme is to monitor and assess potential safety concerns. Data regarding physical safety concerns and quality concerns are shared with local UK Trading Standards authorities. As part of the EU Directive the MHRA is also collecting data via a notification scheme. This scheme ensures quality and consistency of the products on the UK market. The regulation of these products has been in place for a year and this study reviews the data collected to date.

Methods: Traditional signal prioritisation and detection methods have been used to analyse the reports received for potential emerging signals or safety concerns. Comparisons in safety profile have been made to traditional nicotine replacement therapy medicinal products.

Results: Almost 40,000 e-cigarette products have been submitted via the notification scheme including details of nicotine strength, product ingredients and emissions data. In the first year, we have received 18 reports of adverse reactions, 2 reports of physical product concerns and 3 e-liquid quality complaints associated with e-cigarettes via the Yellow Card Scheme. No new safety concerns have yet been identified however this data contributes to the growing evidence about safe use of these products. Discussion/Conclusion: Spontaneous reporting is a vital component in monitoring the safety of e-cigarettes to identify potential adverse reactions or risks to particular population groups using these products. The main principals of pharmacovigilance are directly applied to the analysis of this data and regulatory action such as labelled warnings and patient communication can then be used as appropriate. Reporting in the first year has been low. It is recognised that like spontaneous reporting of ADRs for traditional medicines, communication and public awareness campaigns are needed to promote reporting of adverse effects associated with e-cigarettes to the Yellow Card Scheme.

Further sources of information/References:

1. EU Tobacco Directive. Revision of the Tobacco Products Directive 2014; Available from: http://ec.europa.eu/health/tobacco/products/ revision/index en.htm

2. E-cigarettes: an evidence update A report commissioned by Public Health England. August 2015. Available from: https://www. gov.uk/government/uploads/system/uploads/attachment_data/file/ 457102/Ecigarettes_an_evidence_update_A_report_commissioned_ by_Public_Health_England_FINAL.pdf

\section{Examination of the Trends in Medication Error Reporting Post Legislation Changes in the United Kingdom}

\author{
$\underline{\text { G. Ragunathan }^{1}}$, M. Jadeja ${ }^{1}$, R. Owen ${ }^{1}$ \\ ${ }^{1}$ Medicines and Healthcare Products Regulatory Agency, London, UK
}

Introduction: Pharmacovigilance legislation changes in July 2012 resulted in the expanded definition of a suspected Adverse Drug Reaction (ADR) to include overdose, off-label use, misuse, abuse and medication errors. This brought a great focus on harm associated with a medicinal product outside of its licenced indications to capture new and important safety information. It also highlighted the need for close interaction with 
other patient safety bodies in the UK to ensure clear messages for healthcare professionals and the general public. In March 2014, a joint Patient Safety Alert was issued with NHS Improvement requiring the National Health System to act to improve reporting and strengthen local learning of medication errors. The PSA required the establishment of a National Medication Safety Network [1]. To further facilitate the capture of medication error the MHRA's Yellow Card Scheme online reporting form was updated to includes a field where reporters can state if they feel the reaction they experienced was due to a medication error.

Aim: To examine ADR reporting trends post legislation changes including the impact of joint initiatives with NHS Improvement to increase reporting of medication errors.

Method: Analysis of data received since 2012 from both patients and healthcare professionals via the Yellow Card Scheme and from data received from hospital risk management systems through a weekly data sharing agreement with NHS Improvement.

Results: The number of reports of ADRs arising from medication errors has increased by $95 \%$ since 2012 (table 1). The establishment of Medication Safety Officers and a national medication safety network have directly contributed to the identification of drug safety signals and provided greater detail on the root cause of harms from medication errors. For example poor medication storage of an anticoagulant leading to increased lack of efficacy and cases of overdose arising from lack of visibility of transdermal opioid patches. Live monthly web events and conferences have improved awareness about reporting and the quality of reports and fostered stronger relationships with healthcare professionals.

Conclusion: Our data has shown that reporting of medication errors has nearly doubled in the past 5 years. Collaboration with another patient safety organisation and increased communication with Medication Safety Officers is an important factor behind this increase. Further engagement and communication is still needed to continue to improve the quality of the reports and facilitate quick and easy reporting for the early detection of safety issues associated with medication errors.

Table 1: The number of suspected ADR reports received between 2012-2016 associated with medication errors.

\begin{tabular}{|l|l|}
\hline Year & Medication errors \\
\hline 2012 & 511 \\
\hline 2013 & 753 \\
\hline 2014 & 856 \\
\hline 2015 & 857 \\
\hline 2016 & 999 \\
\hline
\end{tabular}

Further sources of information/References:

1. Cousins D, Gerrett D, Richards N, Jadeja M. Initiatives to Identify and Mitigate Medication Errors in England. Drug Saf 2015;38:349-57

\section{Relevance of safety data in the withdrawal decision of hepatotoxic drugs}

\author{
$\underline{\text { S. Babai }}{ }^{1}$, H. Le Louet ${ }^{1}$ \\ ${ }^{1}$ Pharmacovigilance, Créteil, France
}

Background: Drug induced liver injury (DILI) is a significant concern in marketing failure of commercialized drugs although preclinical and clinical testing are designed to determine if hepatotoxicity can be monitored clinically. Over the years, among drugs withdrawn worldwide, it has been estimated that one third has involved hepatotoxicity $[1,2]$. An analysis of safety information among the drugs withdrawn for hepatotoxicity should be useful in determining whether the prediction of hepatic lesions has improved.

Aim: Analyze and compare drugs safety information from preclinical research to their withdrawals for hepatotoxicity in EU and in US from 1997 to 2016.

Methodology: Drugs withdrawn for hepatotoxicity over the last 20 years in Europe and/or in USA either by marketing authorization holders or by Regulatory agencies have been selected.

Withdrawal decisions were identified from a search within the EMA website, the FDA orange book and PubMed.

Results: From 1997 to 2016, 8 drugs were withdrawn from the market for hepatotoxicity reasons: tolcapone, troglitazone, trovafloxacin, bromfenac, nefazodone, ximelagatran, lumiracoxib and sitaxentan. All these drugs have shown liver abnormalities during clinical trials while most of preclinical studies did not detect liver injury. About tolcapone, trovafloxacin, bromfenac, lumiracoxib and sitaxentan cases, many factors have been probably contributed to their withdrawn: inappropriate monitoring frequency, poor compliance in transaminase monitoring and inappropriate liver function test for this kind of drug.

Discussion/ Conclusion: Over the years, predictive animal models for DILI have not been improved and clinical trials are not too much considered. Among these 8 drugs, unpredictable occurrence and irreversible outcome of hepatotoxicity are the most common reasons of drug withdrawal because the means used in the risk prevention are useless. Therefore the hepatotoxic risk is considered "unacceptable". To become acceptable, the risk must be closely managed with relevant means of prevention. Poor monitoring compliance, inappropriate frequency in transaminase monitoring and little value assigned to rare events can lead to an adequate risk management. It seems that changes in Pharmacovigilance legislation with a closer management of drug safety have contributed to the development of new pharmacovigilance data tools and to the improvement of signal detection. This study also reveals that post-marketing surveillance still must be used to identify and better define cases of hepatotoxicity.

Further sources of information/References:

1. Regev A. DILI and drug development: Regulatory perspective. Semin Liver Dis 2014; 34:227-39

2. Onakpoya IJ, Heneghan CJ, Aronson JK. Delays in post marketing withdrawal of drugs to which deaths have been attributed: a systematic investigation and analysis. BMC Med 2015 5; 13:26

\section{Survey on Physicians' Knowledge, Attitude and Practice towards Adverse Drug Reaction (ADR) Reporting in the Middle East}

\author{
$\underline{\text { A. Hegazy }^{1}}$ \\ ${ }^{1}$ Merck, Dubai, United Arab Emirates
}

Introduction: Under-reporting of ADRs is a common phenomenon that may impact patients' safety [1]. It may delay signal detection and cause underestimation of the size of a problem [2]. Knowledge and attitudes of physicians play pivotal role in ADRs reporting [3]. Few studies on ADRs reporting were conducted in the Middle East. 
Objectives: To identify Knowledge, attitudes, and practice among physicians towards ADRs reporting in Middle East, factors affecting and actions to improve reporting.

Methods: An observational cross sectional design using self-administered questionnaire was used for collecting data. Three Countries were selected: Egypt, Saudi Arabia, and United Arab Emirates. A sample size of 100 Physicians allowed calculation of the percent of those who actually reported ADR at $95 \%$ confidence level with $10 \%$ precision. Multistage cluster sample was selected. Collected data were analysed using SPSS20. Qualitative data summarized as percent with $95 \% \mathrm{CI}$; continuous data summarized by means, SD and SE. Correlation Coefficient was used to assess correlations between continuous variables with the calculation of a coefficient of determination (R2) and its CI. Means were compared using pooled t-test. Significance level was set at 0.05 .

Results: 149 physicians (51.4\%) completed the survey. 87\% were not aware of National Reporting Agency and 17\% received ADR training. $89 \%$ have seen patients experiencing ADR while 56\% claimed they reported it. Seriousness $(96 \%)$ and unexpected nature $(90 \%)$ of ADR were the main factors affecting ADR reporting. 79\% would report ADR if they were sure it was related to a particular drug, $76 \%$ with easier method and $72 \%$ would talk with pharmaceutical companies. Awareness and access to forms were the mostly suggested actions to improve reporting. Age, specialty, work setting and training were independent predictors for reporting ADRs.

Conclusion: There are inadequate knowledge and awareness levels among physicians towards reporting of ADRs. Awareness and training would improve the knowledge and attitudes. This study could provide a sound basis for further research for future strategic actions.

Further sources of information/References:

1. World Health Organization. Safety Monitoring of Medicinal Products: Guidelines for Setting Up and Running a Pharmacovigilance Centre. Available at: http://apps.who.int/medicinedocs/en/d/Jh2934e/6.3.html

2. Biagi C, Montanaro N, Buccellato E, Roberto G, Vaccheri A, Motola D. Underreporting in pharmacovigilance: an intervention for Italian GPs. Eur J Clin Pharmacol 2012; 12: 1321-7

3. Santosh K, Ragulpiankit P. Pharmacovigilance: An Overview. Mahidol Univ J Pharm Sci 2011; 38:1-7

\section{Does the corossol fruit really treat cancer?}

\section{S. Skalli ${ }^{1}$, R. Soulaymani Bencheikh ${ }^{2}$}

${ }^{1}$ Faculty of Science. Mohammed V University Of Rabat, Morocco, Rabat, Morocco, ${ }^{2}$ Centre Anti Poison et de Pharmacovigilance du Maroc, Rabat, Morocco

Background: A pharmacovigilance centre is an organisation that monitors the adverse effects of health products as well as responds to, and provides information on, all safety-related issues and effectiveness of these health products. Pharmacovigilance is applied to all types of health products including herbal medicines (HM). In the case of HM, some patients use these products in the belief that they are safer than prescription medicines, because they are natural. HM are also often used by patients with serious disease, such as cancer.

Objective: To conduct a literature review and synthesis in relation to the use of corossol by the patients with cancer and to highlight the role of pharmacovigilance centres in providing information to patients.

Methods: The Moroccan Pharmacovigilance Centre (CNPV) has received requests from patients with cancer in relation with the safety and the efficacy of corossol (Annona muricata, Annonaceae) fruit. This HM has been recommended via the internet. As the Moroccan database and the Uppsala Monitoring Centre VigiBase ${ }^{\circledR}$ do not contain any adverse reaction reports in relation to the use of this fruit, we carried out a PubMed literature search on this fruit, its composition, its efficacy, its effectiveness, and its risk.

Results and Discussion: Indeed, information about this use is available on the internet that states "the SourSop or the fruit from the corossol tree is a miraculous natural cancer cell killer 10000 times stronger than chemotherapy". Our study is underway but the first results suggest that corossol does nothing special in the human body to hasten a cure or any other such thing with regard to cancer. Furthermore, there are several neurotoxic acetogenins in fruits in the Annonaceae family of plants. These compounds can cause neurotoxic effects such as an atypical form of Parkinson's disease.

Conclusion: The use of HM always requires caution and must be based on well-founded scientific data, particularly if the information on this use reaches us via the Internet where commercial interests may outweigh the interest in promoting health.

\section{Fever, eosinophilia, and abnormal liver function are early signs suggestive of DRESS: a comparative study between DRESS and MPE}

\author{
CY. Chu $^{1}$, SH. Hsu ${ }^{1}$ \\ ${ }^{1}$ National Taiwan University Hospital, Taipei, Taiwan
}

Background: There is a rising awareness of drug reaction with eosinophilia and systemic symptoms (DRESS) due to its possible morbidity and mortality [1-4]. Early diagnosis of DRESS is crucial for administering timely treatment; however, prompt diagnosis based on its early presentation can be quite problematic due to its clinical resemblance to common maculopapular eruptions (MPE).

Objective: To identify the clinical symptoms and signs that are highly predictive of a diagnosis of DRESS in its early stage and thus could differentiate it from MPE in real world clinical practice.

Methods: A retrospective cohort study of patient data from September 2010 to June 2016 was conducted to compare the clinical presentations of DRESS and MPE validated by the RegiSCAR scoring system. The demographic data, clinical presentations, and histopathological patterns were reviewed.

Results: Fifty-eight patients with DRESS and 29 patients with MPE were included. The mean age at diagnosis of DRESS was 47 years (range: 2-82 years), and female patients predominated by a ratio of 2.2:1. The three most common culprit medications for DRESS were allopurinol, sulfasalazine, and trimethoprim/sulfamethoxazole. The most significant differences between the DRESS and MPE groups were the presence of fever, peripheral blood eosinophilia and atypical lymphocytosis, characteristic skin lesions, abnormal liver functions, and prolonged resolution of skin lesions for more than 15 days in the DRESS patients. The most common histologic features in the DRESS patients were coexistent eczematous, interface dermatitis, and vascular damage patterns, or interface dermatitis alone. The concurrence of fever, peripheral blood eosinophilia, and abnormal liver function within three days of visiting a medical facility were more common in cases of DRESS than of MPE (24.1\% vs. $0 \%$, $P=0.004)$.

Conclusion: Although DRESS and MPE look similar, especially in the early stage of DRESS, the concurrence of fever, peripheral blood eosinophilia, and abnormal liver functions within three days of visiting a medical facility might aid in the early diagnosis of DRESS 


\section{Further sources of information/References}

1. Chen YC, Cho YT, Chang CY, et al. Drug reaction with eosinophilia and systemic symptoms: A drug-induced hypersensitivity syndrome with variable clinical features. Dermatol Sinica 2013; 31: 196-204

2. Kardaun SH, Sekula P, Valeyrie-Allanore L, et al. Drug reaction with eosinophilia and systemic symptoms (DRESS): an original multisystem adverse drug reaction. Results from the prospective RegiSCAR study. Br J Dermatol 2013; 169: 1071-80

3. Chen YC, Chang CY, Cho YT, et al. Long-term sequelae of drug reaction with eosinophilia and systemic symptoms: a retrospective cohort study from Taiwan. J Am Acad Dermatol 2013; 68: 459-65

4. Chen YC, Chiang HH, Cho YT, et al. Human herpes virus reactivations and dynamic cytokine profiles in patients with cutaneous adverse drug reactions -a prospective comparative study. Allergy 2015; 70: 568-75

\section{Launch of pilot reporting scheme for harms associated with new psychoactive substances}

\author{
A. Smith ${ }^{1}$, S. Vaughan ${ }^{1}$ \\ ${ }^{1}$ Medicines and Healthcare Products Regulatory Agency, London, UK
}

Introduction: New Psychoactive Substances (NPS) pose potentially serious risks to public health. There has been rapidly increasing numbers of new substances identified in recent years with greater availability over the internet. Hospital admissions for poisoning by 'Psychostimulants with abuse potential' have increased by $45 \%$ in England and Wales from the period 2009-2010 to 2014-2015 [1]. At present evidence is lacking about the long term harms to health associated with use of such illicit substances and more monitoring in this area is needed. The MHRA have launched, in partnership with Public Health England (PHE), a one year pilot reporting website for healthcare professionals across the UK to report harms experienced by patients associated with the use of NPS.

Aim: To describe the establishment of the pilot and review the reports submitted in the first month.

Methods: The new reporting system, Report Illicit Drug Reactions (RIDR) https://report-illicit-drug-reaction.phe.gov.uk/ was modelled on the UK's Yellow Card Scheme to offer a simple reporting process for healthcare professionals. Experts from PHE's Clinical Network on NPS were consulted to identify any additional requirements for capturing harms associated with NPS. Potential challenges with data collection on harms for NPS were also considered such as difficulty in identifying the products used. Key performance indicators for the success of the project were documented and will be reviewed on a quarterly basis by MHRA and PHE.

Results: The RIDR website was launched in March 2017, in the first month of the pilot, 35 reports were reported to RIDR. These reports included a range of different NPS, such as synthetic cannabinoids, psychodelics and fentanyl analogues, as well as reports for other illicit drugs such as cocaine and MDMA. The MHRA has carried out signal detection activities on these reports using the same principles as for licensed medicinal products and are providing fortnightly reports on the data to PHE for review by their network of clinical experts in NPS alongside other NPS intelligence sources.

Discussion/Conclusion: This is the first time that systematic data for harms associated with NPS is being collected and so far the number of reports received in the first month is encouraging. Ultimately we hope that the data collected through this project will lead to the identification of safety signals associated with the use of NPS and licensed medicines. This in turn will help to generate key messages for healthcare professionals to support their patients.

Further sources of information/References:

1. Health and Social Care Information Centre. Hospital admissions for poisoning by illicit drugs up by more than 50 per cent in a decade, July 2016

\section{Generation of a novel, personalised in vitro model to assess the impact of mitochondrial DNA variation upon susceptibility to hepatotoxicity}

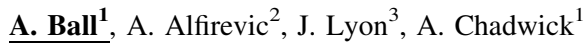 \\ ${ }^{1}$ MRC Centre for Drug Safety Science, University of Liverpool, \\ Liverpool, UK, ${ }^{2}$ Wolfson Centre for Personalised Medicine, \\ Liverpool, UK, ${ }^{3}$ GlaxoSmithKline, R\&D Ware, UK
}

Background: Idiosyncratic drug-induced liver injury (DILI) represents a significant burden to health organisations and is a major contributor to cases of drug attrition [1]. Mitochondrial dysfunction is increasingly implicated in the onset of idiosyncratic DILI; mitochondria contain multiple copies of their own genome, mitochondrial DNA (mtDNA), which has been found to induce changes in mitochondrial function [2,3]. We hypothesise that inter-individual variation in mtDNA underlies the idiosyncratic nature of some cases of DILI via changes in mitochondrial function which can alter susceptibility to injury.

Aim: To generate personalised in vitro models (transmitochondrial cybrids) which are able to better recapitulate the inter-individual variation in mtDNA experienced in the clinic.

Methods: We have performed next-generation mtDNA sequencing of 384 human DNA samples and identified individuals' mitochondrial haplogroups. This is able to inform the recall of volunteers of known mtDNA haplogroups from which fresh platelets (mtDNA donor cells) can be isolated and fused with HepG2 rho-zero cells (devoid of mtDNA) to generate transmitochondrial cybrids, enabling the phenotypic consequences of mtDNA variation to be studied. Data has been collected to validate the success of this technique via extensive functional (extracellular flux analysis), proteomic (western blot) and genetic (qPCR) characterisation. Results: We have shown that the transformation of HepG2 cells with donor mitochondria is able to provide a personalised in vitro model to represent mitochondrial DNA variation.

Conclusion: To the best of our knowledge, this is the first time transmitochondrial cybrids have been generated from HepG2 cells, a commonly used pre-clinical hepatotoxicity testing cell-line. This offers a novel opportunity to study the effect of variation in mtDNA upon mitochondrial function and susceptibility to hepato-mitotoxicants in a relevant, stable cell line and to use this information to inform the stratification of patients in the future.

\section{Further sources of information/References:}

1. Leise MD, Poterucha JJ, Talwalkar JA. Drug-induced liver injury. Mayo Clin Proc 2014;89:95-106

2. Boelsterli UA, Lim PL. Mitochondrial abnormalities-a link to idiosyncratic drug hepatotoxicity? Toxicol Appl Pharmacol 2007;220:92-107

3. Kenney MC, Chwa M, Atilano SR, Falatoonzadeh P, Ramirez C, Malik D, et al. Molecular and Bioenergetic Differences between Cells with African versus European Inherited Mitochondrial DNA Haplogroups: Implications for Population Susceptibility to Diseases. Biochim Biophys Acta 2014;1842:208-19 
Abbreviations: DILI; drug-induced liver injury, mtDNA; mitochondrial DNA,

\section{Evaluation of the risk of Denosumab-Associated Hypocalcaemia}

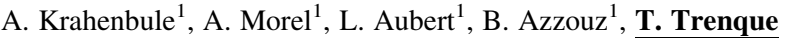 \\ ${ }^{1}$ Regional Center of Pharmacovigilance and Pharmacoepidemiology, Reims, France}

Background: Denosumab is a human monoclonal antibody working through inhibition of RANK-ligand osteoclast-mediated bone resorption. As such, it is used to prevent complications of osteoporosis, bone metastasis and giant cells tumors. However, a trade-off from this action is an increased risk of occurrence of denosumab-associated hypocalcaemia (DAH), which can lead to serious complications.

Objective: The present study aimed to evaluate the risk of hypocalcaemia associated with the intake of denosumab.

Method: All cases of adverse drug reactions including hypocalcaemia with denosumab reported to the French Pharmacovigilance Database from 1985 to the 31st of March 2017 were reviewed. Demographic and medical information were analyzed. Renal failure, a known risk factor for DAH, also was documented. The association between reported cases of hypocalcaemia and denosumab use was assessed using Reporting Odds Ratio (ROR) with 95\% confidence interval.

Results: 543 cases of hypocalcemia were collected in the database of which 53 with denosumab. Patients had a mean age of 70.36 years, males and females were represented in similar proportions ( 27 females, 25 males and 1 unknown). Concerning risk factors, renal failure was identified in 22 cases $(41.5 \%)$ and severe renal failure (defined as a renal creatinine clearance inferior to $30 \mathrm{ml} / \mathrm{min}$ ) in 7 cases $(13.2 \%)$. Hypocalcaemia was significantly associated with the use of denosumab, ROR $=191.93$ [142.04-259.33].

Conclusion: This study, performed on a large national pharmacovigilance database, implies the existence of a very significant risk of hypocalcaemia associated with the use of denosumab. Hypocalcaemia is a known side effect of denosumab, however, the extreme value of ROR found in this study implies its potential underestimation. Hypocalcaemia can lead to serious complications and a risk-benefit ratio assessment, as well as close monitoring of patients under denosumab, especially if they suffer from renal failure, is strongly recommended.

\section{Evaluation of the first EU-wide social media ADR awareness week: 7-11 November 2016}

\section{Jadeja ${ }^{1}$ \\ ${ }^{I}$ MHRA, London, UK}

Background: A Strengthening Collaboration for Operating Pharmacovigilance in Europe (SCOPE) Joint Action project survey found only four EU National Competent Authorities (NCAs) used social media to promote ADR reporting [1]. A key deliverable from this SCOPE awareness levels topic, led by the Medicines and Healthcare products Regulatory Agency (MHRA), was to develop a media toolkit to support NCAs promote suspected ADR reporting nationally. Underreporting is a known limitation of spontaneous ADR reporting systems and is linked to awareness levels.
Objective/Aim: To develop and run an EU-wide social media campaign to increase ADR reporting by $5 \%$ using SCOPE materials and measure its effectiveness to provide insight into future campaigns [2].

Methods: SCOPE developed a media toolkit of campaign collateral for NCAs consisting of animations and infographics [3,4]. The ADR awareness week took place between 7 and 11 November 2016. To support NCAs, a template press release; campaign guide; and a running plan were developed and shared. NCAs were able to adapt to suit these to their own needs. The campaign plan was developed and led by MHRA and formalised via the Heads of Medicines Agency Working Group for Communications Professionals (HMA WGCP). Participation was encouraged through SCOPE meetings and HMAWGCP. Social media channels used: LinkedIn, Facebook, YouTube, Twitter and websites. Audiences included patients, public and healthcare professionals. Participating NCAs monitored campaign impact and reported their findings to MHRA, including changes reporting compared to a baseline.

Results: 21 NCAs participated with common ADR messages. SCOPE produced 24 different versions of tailored collateral in different languages for NCAs and stakeholders such as the European Medicines Agency, the European Commission and patient organisations. Between 15 NCAs there was a $13 \%$ increase in suspected ADR reporting (1056 reports). The campaign reached 2562,071 people; 337,781 viewed animation; 22,584 likes, clicks, retweets and shares. A multitude of stakeholders were engaged to disseminate messages. Feedback on what worked well and improvements were analysed. All respondents indicated it was worthwhile running.

Conclusion: The SCOPE ADR awareness week campaign was a significant success despite being the first of its kind. Recommendations include consideration of more frequent social media use and further working together between NCAs to make this an annual ADR awareness week at a global level to encourage suspected ADR reporting for an improved and a larger data pool for signal detection.

Further sources of information/References:

1. Jadeja M, Barrow P. Increasing Awareness of National Adverse Drug Reaction Reporting Systems: Survey Report, 2015. Accessible: http://bit.ly/2qbJqSh

2. SCOPE Awareness levels toolkit. Accessible: http://bit.ly/2qB93xf

3. SCOPE animation: http://bit.ly/2qefC5P

4. SCOPE infographics: http://bit.ly/2qebSBg

\section{Mechanisms of Chemotherapy-Induced Diarrhoea}

\author{
$\underline{\text { S. French }^{1}}$, A. Davies ${ }^{1}$, M. Pirmohamed ${ }^{1}$ \\ ${ }^{1}$ University of Liverpool, Liverpool, UK
}

Background: Lower gastrointestinal toxicity-which presents clinically as diarrhoea and colitis-is a prevalent adverse event occurring during chemotherapeutic treatment. In addition to decreasing treatment efficacy and patient quality of life, it contributes to the economic burden on the NHS due to patient hospitalization and subsequent complications. Tyrosine kinase inhibitors are a broad class of chemotherapeutic agents for which this is a major problem. For example Bcr-Abl inhibitors used for the treatment of chronic myeloid leukaemia induce diarrhoea with a frequency of 70 and $25 \%$ for bosutinib and imatinib, respectively [1]. However, the mechanistic basis of this remains poorly understood.

Objective/Aim: Determine the processes involved in Bcr-Abl inhibitorinduced diarrhoea

Methods: Caco-2 (human colorectal cancer) cells, which differentiate into monolayers of polarised enterocytes, were utilized as an in vitro model. 
Cells were seeded into transwells which enable changes in paracellular permeability to be determined by measuring electrical resistance and flux of FITC-dextran, a fluorescently labelled polysaccharide, across the monolayer. Changes in gene expression, protein levels and protein localization were studied using RTqPCR, immunoblotting and immunocytofluorescence, respectively.

Results: Sub-apoptotic concentrations of bosutinib increased paracellular permeability of Caco-2 monolayers to ions and FITC-dextran (ANOVA, $\mathrm{p}<0.0001$ ), whilst imatinib was less effective at inducing this change (ANOVA, $\mathrm{p}<0.001$ ). Bosutinib decreased protein levels (ANOVA, $\mathrm{p}<0.05$ ), but not RNA levels, of cell junction protein E-cadherin-a transmembrane protein already implicated in tyrosine kinase inhibitorinduced diarrhoea ${ }^{[2,3]}$. In addition, mislocalization of E-cadherin was observed after bosutinib treatment. These changes were not seen for imatinib. As ER stress is involved in E-cadherin degradation induced by another tyrosine kinase inhibitor (erlotinib) [3], the role of ER stress in bosutinib-induced E-cadherin decrease was investigated. However, no increase in ER stress markers were detected after bosutinib or imatinib treatment.

Conclusion: Decreased intestinal barrier integrity, potentially mediated by E-cadherin degradation, is likely an important factor in the aetiology of bosutinib-induced diarrhoea. This information will provide useful for identification and development of better treatments for this gastrointestinal adverse event.

Further sources of information/References

1. Brümmendorf T, Cortes J, de Souza C, Guilhot F, Duvillié L, Pavlov $\mathrm{D}$, et al. Bosutinib versus imatinib in newly diagnosed chronic-phase chronic myeloid leukaemia: results from the 24-month follow-up of the BELA trial. Br J Haematol 2015;168:69-81

2. Hong S, Gu Y, Gao Z, Guo L, Guo W, Wu X, et al. EGFR inhibitordriven endoplasmic reticulum stress-mediated injury on intestinal epithelial cells. Life Sci 2014;119:28-33

3. Fan L, Hu L, Yang B, Fang X, Gao Z, Li W, et al. Erlotinib promotes endoplasmic reticulum stress-mediated injury in the intestinal epithelium. Toxicol Appl Pharmacol 2014;278:45-52

\section{Whole exome sequencing in individuals with statin- induced myopathy}

K.M. Bloch ${ }^{1}$, D. Carr $^{1}$, M. Pirmohamed ${ }^{1}$, A. Morris ${ }^{1}$, C.

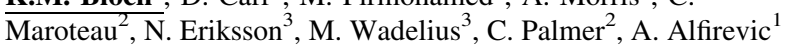

${ }^{1}$ University of Liverpool, Liverpool, UK, ${ }^{2}$ Dundee University, Dundee, UK, ${ }^{3}$ Uppsala University, Uppsala, Sweden

Background: Statins are widely used lipid-lowering drugs that are effective in reducing cardiovascular disease risk. Although they are safe and effective in the majority of patients, they can cause muscle toxicity. The clinical spectrum of statin-induced myotoxicity varies from asymptomatic elevations of creatine kinase (CK), to muscle pain or weakness with raised CK levels and rhabdomyolysis. The precise mechanisms underlying statin myotoxicity are unknown, but several hypotheses have been suggested including alterations in myocyte membrane cholesterol, depletion of isoprenoids, depletion of ubiquinone or coenzyme Q10 and genetics such as a strong association between simvastatin-associated myopathy and the common polymorphisms in SLCO1B1, a solute carrier organic anion transporter has been identified [1].

Objective: The objective of the study is to try to identify genetic risk factors that are responsible for statin-induced myopathy.
Methods: We standardized nomenclature (SRM1-SRM6) and phenotypic definitions of statin-induced muscle toxicity [2] and recruited 779 Caucasian patients on statins (Table 1). Most of our patients were on simvastatin $(\mathrm{N}=511 ; 66 \%)$ and atorvastatin $(\mathrm{N}=201 ; 26 \%)$. We performed exome sequencing in 779 patients; 245 with statin-induced toxicity (SRM3-SRM6) and 534 tolerant controls. DNA libraries from each sample were pooled for exome capturing using the SureSelect v5 all-exon probe set (Illumina). Sequencing was performed with paired-end $2 \times 125$ base reads on the Illumina HiSeq 2500 platform. The data was aligned to indexed reference genome hg19/GRCh37 using BWA, indel realignment, base quality score recalibration and variant calling were performed using Genome Analysis Toolkit (GATK) v.3.5 HaplotypeCaller. The single variant and gene-based analyses were performed using EPACTS.

Results and Conclusion: We have identified IPO9, CELF2, SIN3A and DOCK1 as top significant genes. Future work will include validation of the findings and a collaborative meta-analysis of whole exome sequencing with the US cohort.

This work was funded by the EU.

\begin{tabular}{|l|l|l|l|l|}
\hline & Mild (SRM 3) & Severe (SRM4, SRM5) & Autoimmune (SRM 6) & TOTAL \\
\hline Case & 146 & 91 & 8 & 245 \\
\hline Control & NA & NA & NA & 534 \\
\hline TOTAL & 146 & 91 & 8 & 779 \\
\hline
\end{tabular}

\section{Further sources of information/References}

1. Link E, Parish S, Armitage J, et al. SEARCH Collaborative Group SLCO1B1 variants and statin-induced myopathy-a genomewide study. N Engl J Med 2008;359:789-99

2. Alfirevic A, Neely D, Armitage J, Chinoy H, Cooper RG, Laaksonen R, Carr DF, Bloch KM, Fahy J, Hanson A, Yue QY, Wadelius M, Maitland-van Der Zee AH, Voora D, Psaty BM, Palmer CN, Pirmohamed M. Phenotype standardization for statin-induced myotoxicity. Clin Pharmacol Ther 2014 Oct;96:470-6.

\section{A Description of New Safety Signals Detected and Assessed by PRAC at EU level in 2014-2016}

\author{
A. Mahalean ${ }^{1}$, A. Farcas ${ }^{1}$ \\ ${ }^{I}$ Drug Information Research Center, University of Medicine \\ and Pharmacy, Cluj-Napoca, Romania
}

Background: Safety monitoring of all drugs throughout their entire lifecycle, in order to assure that the benefit-risk balance remains positive, is mandatory in order to protect the public health. New potential risks or changes in known risks arising from different sources are evaluated through the signal management process. At EU level the Pharmacovigilance Risk Assessment Committee (PRAC) is mandated to assess signals and make recommendations for action following the signal assessment. Objective: To describe all new signals detected and assessed at EU level in 2014-2016.

Methods: Publicly available data on signals assessment and prioritization from PRAC meeting minutes for the period January 2014-December 2016 were collected, analyzed and classified according to predefined criteria (e.g. drug evaluated, type of signal, signal source, PRAC final recommendation). Results: 190 new signals for 157 drugs/drugs associations/therapeutic classes, were evaluated by PRAC in this study's timeframe. 122 new signals 
were triggered by spontaneous reporting, 31 by literature monitoring, 2 by randomized controlled trials, or by other sources. Most drugs evaluated were authorized through CAP $(n=135)$. In 41 of the newly detected signals the drugs evaluated were authorized in the last 5 years. The drug classes for which most signals were detected and assessed were antineoplastic and immunomodulators $(n=58)$, anti-infective drugs for systemic use $(n=24)$ and drugs acting on the nervous system $(n=24)$. Signals were triggered for drug interactions $(n=9)$, in utero exposure $(n=6)$, medication errors $(n=3)$, off label use $(n=1)$ and for other different disorders, among which the blood and lymphatic system and skin and subcutaneous tissue disorders were more common. PRAC recommendations consisted in summary of product characteristics (SmPC) updates in 57 cases, package leaflet (PIL) updates in 50 cases, in sending a Direct Healthcare Professional Communication in 12 cases, Risk Management Plan updates in 11 cases, and 1 recommendation to start a referral. There were 10 cases in which no regulatory action was considered necessary. For other signals PRAC recommendation was for routine pharmacovigilance activities and discussion of the signal within the next Periodic Safety Update Report. 17 signals were still ongoing at the time of this study.

Conclusions: Most new signals assessed in the study period were triggered by spontaneous reporting and led to routine pharmacovigilance risk minimisation measures, such as updating the SmPC and the PIL. This assessment outlines again the importance of the spontaneous reporting system for up to date, safe and rational prescribing information.

\section{Drug-Injury Relief System for Patient Benefit in Taiwan: the 20 years of experience}

\author{
W. Chen ${ }^{1}$, W. Lun $\mathrm{Wu}^{1}, \mathrm{M} . \mathrm{Chu}^{1}$ \\ ${ }^{1}$ Taiwan Drug Relief Foundation, Taipei City, Taiwan
}

Background: The knowledge of science and technology cannot guarantee $100 \%$ drug safety for all users under proper usage circumstances nowadays. To benefit those victims who have not sought compensation via general lawsuit, the no-fault drug-injury relief system in Taiwan has been implemented for almost 20 years by Taiwan Drug Relief Foundation to help drug injury victims to get prompt help ${ }^{(1-2)}$. This article reviewed this unique no-fault compensation-based system. We hope the fruitful results of the system will be references for other countries sought for plan such similar system for patients' benefit.

Objectives/aim: This article is aimed to review the drug-injury relief system in Taiwan and to analyze the achievements around 20 years of implementation.

Methods: This research applies quantitative and qualitative methods to collective data from Taiwan Drug Relief Database.

Results: The results showed that the trend of annual application for relief has risen from less than 100 to close to 200 cases; the total approval rate has risen from less than 40 to 58\%. Among all applications, the most frequently applied ADRs are skin and subcutaneous disorders (67\%) where SJS and TEN are associated with higher risk of death or disability. The most frequent culprit drug of SJS/TEN are anticonvulsants (carbamazepine, phenytoin), antibiotics, non-steroidal anti-inflammatory drugs, and allopurinol.

Conclusions: The spirit of Taiwan Drug Relief System is based on consumer protection which leads to the trend of higher application approval rate. The ultimate goal of the system is to prevent further drug injuries from occurring. To achieve the goal, the most commonly applied class of ADR is focused to better communicate with both the public and health care providers. ADR education materials such as Drug Allergy Card and Early Symptoms of Drug Allergy Self-examination Table are provided as communication tools which assist medical professionals with high quality care and effective communication to their patients. Serious skin reaction is therefore early detected and prevented.

Further sources of information/References:

1. Drug Injury Relief Act. 2011.

2. On AWF, et al. Unique Drug-Injury Relief System in Taiwan: Comparing Drug-Injury Compensation in Different Countries. J Pharm Health Serv Res 2012; 3: 3-9

\section{Reintroduction of reference infliximab product in patients showing inefficacy to its biosimilar}

\author{
S. Babai ${ }^{1}$, W. Akrout ${ }^{2}$, H. Le-Louet ${ }^{1}$
}

\section{${ }^{1}$ Pharmacovigilance, Créteil, France, ${ }^{2}$ Pharmacy, Créteil, France}

Background: Biosimilars are biologic medicines that enter the market after a patent for an original reference product expires. In Europe, interchangeability is the medical practice of changing from one medicine to another that is expected to achieve the same clinical effect. Since May 2016, according to the European guidelines, the French Health Authorities have allowed interchangeability between a biotherapy reference product and its biosimilar.

Although many publications have shown that preliminary evidence supports the biosimilarity and interchangeability of biosimilar and reference TNF- $\alpha$ inhibitors, a series of cases of biosimilar infliximab inefficacy have been reported in a French department. Taking these results into account, the need for infliximab reference product (IRP) reintroduction is therefore raised for these patients.

Aim: To assess the reintroduction of IRP in adult patients with spondyloarthritis after inefficacy of its infliximab biosimilar.

Methodology: This is a retrospective study conducted from June to December 2016 on patients with spondyloarthritis switched from IRP to its biosimilar in a real-life clinical setting. Transition monitoring was mainly based on the occurrence of arthralgia and the occurrence of partial or total efficacy loss.

Transition was considered as unsuccessful after 2 biosimilar infusions with patient complain.

For patients showing drug failure with infliximab biosimilar, IRP was reintroduced.

Results: Among 53 patients with spondyloarthritis switched from IRP to its biosimilar,12 patients did not reach the 3rd biosimilar administration. Spondyloarthritis patients reported the occurrence of arthralgia (12/12) and a partial (8/12) or total (4/12) efficacy loss.

For all patients showing drug failure with infliximab biosimilar, IRP was systematically reintroduced.

Among these 12 patients, 7 showed a re-established efficacy, 2 patients did not show clinical improvement and no follow-up information were provided for the 3 last patients.

Discussion/conclusion: This study showed a transition failure rate at $23 \%$ (12/53) after the switch from IRP to its biosimilar for patients with spondyloarthritis. In order to complete these results, an anti-IFX antibodies monitoring is in progress so to highlight any biosimilar activity loss.

Although the patient's gobal assessment may be considered as subjective for a primary criterion and the need of further investigations, the 
professional healthcare may consider the reintroduction of IRP in case of inefficacy of its infliximab biosimilar.

\section{Ongoing Elevated Risk of Developing Tuberculosis in Rheumatological Conditions Where Anti-TNF-alpha Agents Were Used: Results of 2-Year Retrospective Cohort Study}

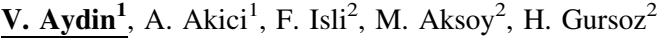 \\ ${ }^{I}$ Department of Medical Pharmacology, School of Medicine, \\ Marmara University, Istanbul, Turkey, ${ }^{2}$ Turkish Medicines \\ and Medical Devices Agency, Ministry of Health, Ankara, Turkey
}

Introduction: We recently showed that 1-year treatment with anti-tumor necrosis factor-alpha (anti-TNF- $\alpha$ ) drugs increased the risk of tuberculosis in patients with certain rheumatologic diseases (RD) [1]. Several studies reported the time to develop tuberculosis from the start of anti-TNF- $\alpha$ therapy up to 15 months $[2,3]$.

Aim: To determine the relative risk of developing tuberculosis during 2-year usage of anti-TNF- $\alpha$ in RD patients.

Material and Method: This retrospective cohort study included patients with RDs (rheumatoid arthritis, ankylosing spondylitis, juvenile arthritis, and psoriatic arthritis) that were treated with or without anti-TNF- $\alpha$ agents. These patients were followed for two years after being registered to the Prescription Information System of the Turkish Medicines and Medical Devices Agency between 1 April 2013 and 31 December 2013. Two-year relative risk of developing tuberculosis after anti-TNF- $\alpha$ usage was estimated.

Results: We identified a total of 413,500 RD patients who had two years of follow-up data. The number of tuberculosis cases within this cohort was 132, showing an incidence of 31.9 per 100,000 patients. There were 411,383 patients in the control arm (anti-TNF- $\alpha$-naïve RD patients) and 2117 patients in the study arm (who was prescribed anti-TNF- $\alpha$ drug at least twice). Most commonly used anti-TNF- $\alpha$ drugs was etanercept $(42.8 \%)$, followed by infliximab (24.1\%) and adalimumab (20.3\%). Percentages of male patients in control and study arm were $29.7 \%$ and $54.3 \%$, respectively. Mean age of the groups were $52.7 \pm 17.1$ and $41.9 \pm 13.4$, respectively. One hundred and twenty-eight patients in the control arm developed tuberculosis compared with four patients in the study arm (31.1 vs 189.0 cases per 100,000 patients, respectively) with a relative risk of developing tuberculosis of 6.07 (95\% CI, 2.25-16.42). Incidence of tuberculosis in both control and study arm was 2.0 and 2.5 times higher in male patients than that in female patients (48.4 vs 23.9 per 100,000 patients and 260.9 vs 103.4 per 100,000 patients, respectively). Age did not influence the risk of developing tuberculosis within either group.

Conclusion: Based on a nationwide cohort data, this study indicated ongoing elevated relative risk of developing tuberculosis in RD patients who were under anti-TNF- $\alpha$ therapy for a period of two years in Turkey. Further sources of information/References:

1. Akici A, Aydin V, Kadi E, Isli F, Gursoz H. Increased risk of tuberculosis in patients with rheumatologic diseases managed with anti-TNF- $\alpha$ agents: a nationwide retrospective pharmacoepidemiological cohort study in Turkey. Poster presented at the $13^{\text {th }}$ Congress of European Association of Clinical Pharmacology and Therapeutics, Prague, Czech Republic in 24-27 June, 2017

2. Gómez-Reino JJ, Carmona L, Miguel Ángel Descalzo. Risk of tuberculosis in patients treated with tumor necrosis factor antagonists due to incomplete prevention of reactivation of latent infection. Arthritis Care Res 2007; 57: 756-61
3. Tubach F, Salmon D, Ravaud P, Allanore Y, Goupille P, Bréban M, et al. Risk of tuberculosis is higher with anti-tumor necrosis factor monoclonal antibody therapy than with soluble tumor necrosis factor receptor therapy: The three-year prospective French research axed on tolerance of biotherapies registry. Arthritis Rheum 2009; 60: 1884-94

\section{Hemorrhagic stroke after thrombolysis}

\author{
A. Mrani Alaoui ${ }^{1}$, O. Ziraoui ${ }^{1}$, K. Lechheb ${ }^{1}$, N. Doghmi ${ }^{2}$,
} Y. Bousliman ${ }^{1,}$

${ }^{I}$ Pharmacy Department, Military Instruction Hospital Mohammed V, Rabat, Morocco, ${ }^{2}$ Intensive Care Unit, Military Instruction Hospital Mohammed V, Rabat, Morocco

Background: Intracranial hemorrhage is a serious but infrequent complication of thrombolysis associated with heparin therapy after myocardial infarction.

Objective: The aim of this work is to study accountability in patient with Hemorrhagic stroke after thrombolysis.

Methods: We report the case of a 56-year-old smoking patient who presented a table of epigastralgia and vomiting with an episode of syncope. After consultation at the Laayoune Military Hospital, the ECG and troponin dosage were in favor of acute coronary syndrome with ST segment elevation. After elimination of any contraindications the patient underwent a trombolysis using $40 \mathrm{mg}$ of tenecteplase (metalyse) associated with enoxaparin (6000 UI) in two injections, $300 \mathrm{mg}$ of aspirin and $600 \mathrm{mg}$ of clopidogrel. Twenty-four hours later, he was unconscious and presented right hemicorpus deficit with left mydriasis, which motivated the realization of a cerebral CT that objectified a cerebral haemorrhagic stroke of the brain stem

The patient was intubated and ventilated under neurological criteria and transferred by air to the medical intensive care department of the Military Instruction Hospital Mohammed V for treatment. The accountability study was carried out in accordance with the French method.

Results: Using the French method for the tenecteplase / hemorrhagic stroke pair, we have acquired an accountability score of I3B4 with an NI2 level of informativity.

Conclusion: The hemorrhagic risk of thrombolysis is severe. In addition to verifying contraindications, it requires strict adherence to the time between ischemia and fibrinolysis and control of blood pressure.

\section{Profile of Allopurinol toxidermia: a series of 24 cases}

Y. Moutaouakkil ${ }^{1}$, A. Tebaa ${ }^{2}$, R. Soulaymani Bencheikh ${ }^{2}$, Y. Bousliman $^{2}$

${ }^{I}$ Faculty of Medicine And Pharmacy, University Mohammed V In Rabat, Rabat, Morocco, ${ }^{2}$ Center For Antipoison Et Pharmacovigilance of Morocco, Rabat, Morocco

Background: Toxidemia is the dermal side effect of systemically administered drugs. They account for $20 \%$ of the side effects of drugs. Toxidermias are characterized by their clinical polymorphism. Allopurinol is the first xanthine oxidase inhibitor that has been on the market for more than 40 years. While it is generally well tolerated, the severe toxidermas associated with it constitute a major risk during its use. 
Aim: The aim of our study is to describe the clinical and evolutionary characteristics of these allopurinol drug reactions and to determine their etiological profile.

Methods: Retrospective study carried out at the Department of Dermatology of the military instruction hospital Mohammed V, Rabat, collecting all cases of allopurinol toxidermia over a period of two years (January 2014-December 2016). The diagnosis of toxidermia was suspected before the combination of clinical anamnestic elements, clinical and evolutionary. The paraclinic explorations were performed according to the clinical picture. A histological study has been performed for some patients in cases of severe forms or diagnosis. The study of imputability was carried out according to the French method of Begaud based on a set of semiological, chronological and bibliographic criteria.

Results: Twenty-four files were collected (15 women and 09 men), a gender ratio $(\mathrm{M} / \mathrm{F})$ of 0.6.L'âge moyen au moment du diagnostic était de 47,4 ans. Twelve patients in whom allopurinol was prescribed outside of its validated indications ( 08 cases for asymptomatic hyperuricemia and 04 cases for suspicion of gout). Clinical manifestations were urticaria in 4 cases, fixed pigmented erythema in 03 cases, macula-papular rash in 6 cases, eczema in 3 cases. We have identified 6 cases of toxic epidermal necrolysis including two Stevens-Johnson syndrome and four cases of Lyell syndrome. DRESS (Drug Reaction with Eosinophilia and Systemic Symptoms) is represented in 02 cases. Sixteen patients $(66.7 \%)$ were treated with dermocorticoids alone with a favorable outcome, and 8 patients required the use of general corticosteroid therapy. For all patients, allopurinol was discontinued. The trend was favorable in all cases.

Conclusion: Control of the misuse of allopurinol alone could reduce the number of cases of severe toxidemia by half and the patient's warning to detect these serious adverse reactions as quickly as possible and thus With a delay in treatment discontinuation associated with the more severe forms of these toxidermia.

\section{Cardiac toxicity after long-term treatment with chloroquine in a lupus patient}

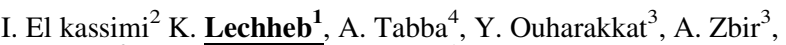 \\ K. Ennibi ${ }^{2}$, J. Chaari ${ }^{2}$, Y. Bousliman ${ }^{1}$ \\ ${ }^{1}$ Pharmacy Departement Hopital Militaire Mohammed V, Rabat, \\ Morocco, ${ }^{2}$ Service de Médecine Interne A, Hôpital Militaire \\ d'Instruction Mohammed V, Rabat, Morocco, ${ }^{3}$ Service de \\ Cardiologie. Hôpital Militaire d'Instruction Mohammed V, Rabat, \\ Morocco, ${ }^{4}$ Centre d'Antipoison et Pharmacovigilance, Hôpital \\ Militaire d'Instruction Mohammed V, Rabat, Morocco
}

Background: Chloroquine (Nivaquine ${ }^{\circledR}$ ) is a synthetic antimalarial (PSA) commonly used in the treatment of connective tissue disorders and is generally well tolerated. The most common side effects are nausea and vomiting at the introduction of the treatment. Muscular, particularly cardiac, potentially severe side effects are often underestimated and there is no recommendation for cardiac monitoring of patients with long-term APS Objective: The objective of this work is to study accountability in a patient with cardiotoxicity following prolonged use of chloroquine

Methods: We report the case of a 54-year-old female patient followed for 10 years for cutaneous and articular lupus treated with chloroquine ( $\mathrm{Ni}$ vaquine ${ }^{\circledR}$ ) at the maximum dose of $300 \mathrm{mg} / \mathrm{d}$, admitted to emergency for syncopal malaise with convulsive seizures generalized.
The electrocardiogram demonstrated ventricular hyperexcitability and a BAV Mobitz II. Clinical examination revealed good hemodynamics, normal cardiopulmonary auscultation and normal neurological examination. Cardiac ultrasound was normal. In contrast, the plasma chloroquine level was $3.6 \mathrm{~mol} / 1$ ( 0.7 to $2.2 \mathrm{~mol} / \mathrm{l})$.

Common biological examinations showed normal renal function, moderate hypokalaemia, moderate hepatic cytolysis

In the case of this patient, to determine the accountability of chloroquine we used the French method and that of the WHO.

Results: The timescales for the drug are compatible.

Evolution is suggestiveThe rechallenge was OIn the literature chloroquine cadiotoxicity is an effect already described

Conclusion: The accountability score according to the French method was I3B3 with a level of informativity of 2. According to the criteria of the WHO method, the occurrence of this adverse effect is likely

\section{Toxic epidermal necrolysis due to betalactamins: 2 case reports}

\author{
A. Mrani Alaoui ${ }^{1}$, O. Ziraoui $^{1}$, A. Meskine ${ }^{2}$, Y. Bousliman ${ }^{1}$, \\ S. Siah $^{2}$
}

${ }^{I}$ Pharmacy departement, Military Instruction Hospital Mohammed V, Rabat, Morocco, ${ }^{2}$ Burns unit, Military Instruction Hospital Mohammed V, Rabat, Morocco

Background: Lyell's syndrome or toxic epidermal necrolysis (TEN) is one of the most serious mucocutaneous drug events, it can be fatal. However, it is rare, with an incidence of $0.1 \%$ of the general population. Objective: The aim of this work is to study accountability in patients with toxic epidermal necrolysis

Methods: We report the findings of two patients with lyell syndrome who were hospitalized in the burns department.

Ms L, 36 years old with rheumatoid arthritis under leflunomide and corticosteroids.

The patient presented with a flu-like syndrome treated with amoxicillin, phenylpropanolamine-carbinoxamine and paracetamol. A week later the patient presented diffuse erythematous skin lesions, with respiratory gene and dry cough. The burned skin surface area was evaluated at $45 \%$.

The interrogation revealed a notion of self-medication with amoxicillin before the flu-like syndrome.

Ms F, aged 69 years, with type 2 diabetes for 10 years, associated with heart failure for 5 years under treatment with furosemide, ramipril and spironolactone.

The onset of symptomatology followed the administration of Cefaclor for ENT infection. The patient presented a skin rash followed by the installation of a generalized skin detachment on an erythematous background. The examination found a generalized epidermal detachment at $82 \%$

The accountability study was carried out in accordance with the French method.

Results: For the first case, amoxicillin was incriminated with an imputability score of I5B4 according to the French method.

In the second case, cefaclor was incriminated with an I3B4 accountability score using the same method.

Conclusion: In order to optimize the detection and the management of this syndrome and to improve the prognosis there are two rules to follow: Early consultation with any post-drug dermatological symptoms. 
Hospitalization of patients with suspicion of Lyell syndrome in a burn department.

\section{ALOPECIA DURING A SPA: WHAT LINK WITH INFLIXIMAB?}

\author{
$\underline{\text { K. Lechheb }}{ }^{1}$, I. El kassimi ${ }^{2}$, A. Tebaa ${ }^{3}$, A. Rkiouak ${ }^{2}$, K. Ennibi ${ }^{2}$, \\ J. Chaari $^{2}$
}

${ }^{1}$ Pharmacy Departement,Hopital Militaire Mohammed V, Rabat, Morocco, ${ }^{2}$ Service de Médecine Interne A, Hôpital Militaire d'Instruction Mohammed V, Rabat, Morocco, ${ }^{3}$ Centre d'Antipoison et Pharmacovigilance, Rabat, Morocco

Background: Biotherapy represents therapeutic advances in the treatment of immunological and inflammatory diseases. However, its use more frequent revealed adverse reactions rarely indicated especially cutaneous, and mainly associated with anti-tumor necrosis factor (TNF) $\alpha$

Objective: The aim of this work is to determine the accountability in a patient with alopecia following the administration of the Influximab

Methods: We report a case of a 36-year-old patient followed since 2007 for ankylosing spondylitis. No improvement was observed in Sulfasalazine and AINS justifying the use of Infliximab. After the 7th cure, the patient developed alopecia mainly involving the scalp whose etiological balance remained negative.

To determine the accountability of this drug, we used the French method of accountability.

Results: The timescales for the drug are compatible.

The progression to stoppage of the drug is inconclusive

The rechallenge was 0

Alopecia is a notorious effect already described in the literature.

Conclusion: The accountability score was I2B4 with an informativity level of 2 .

In our patient, we were tempted to retain Infliximab as responsible for alopecia.

\section{Attitudes of Non-medical Nurse Prescribers towards Reporting Adverse Drug Reactions via the Yellow Card Scheme-an Exploratory Study}

\author{
A. Abbas ${ }^{1}$, C. Green ${ }^{1}$, R. Mullen ${ }^{2}$ \\ ${ }^{1}$ Countess of Chester Hospital NHS Foundation Trust, Chester, UK, \\ ${ }^{2}$ School of Pharmacy and Biomolecular Sciences, Liverpool John \\ Moores University, Liverpool, UK
}

Background: Under-reporting of Adverse Drug Reactions (ADRs) is a major public health concern globally and is a major limitation of spontaneous reporting systems. The Yellow Card Scheme (YCS) is the principal system for reporting ADRs in the UK. Only 6 $-10 \%$ of serious ADRs are reported through the YCS and underreporting of ADRs by healthcare professionals is a key contributing factor [1]. ADR reporting by nurses including non-medical nurse prescribers (Nurse NMPs) at the Countess of Chester Hospital $(\mathrm{CoCH})$ is currently poor.

Aims: To ascertain the level of awareness of Nurse NMPs at $\mathrm{CoCH}$ about the YCS, explore their attitudes towards pharmacovigilance and identify related training needs and barriers to improve ADR reporting rates.

Methods: The first phase was qualitative. Semi-structured interviews were conducted with a purposive sample of 11 nurse NMPs from different specialities recruited by e-mail invitation. Participants who had an opportunity to attend a recent Yellow Card Centre North West (YCCNW) pharmacovigilance training session were asked about their views on content. The findings were analysed thematically using NVIVO 11 software until thematic saturation was reached. The second phase was quantitative and aimed to observe whether pharmacovigilance training offered would improve ADR reporting by Nurse-NMPs at $\mathrm{CoCH}$.

Results: Participants were aware of the YCS as a scheme but were not competent in using it because of gaps in knowledge about who can or should report ADRs, ADR reporting criteria and the available reporting methods. Indifference towards ADR reporting was recorded and the reasons listed were lack of feedback to reporters and misconceptions that the process is time consuming without ultimately making a difference to patient care. Some participants assumed that certainty must be established before ADRs are reported. These factors are modifiable through education. Practical barriers to ADR reporting were the difficulty in identifying ADRs partly as a result of narrow personal formularies, absence of reminders and the inefficiency of reporting systems in facilitating reporting at convenient times. YCCNW training material was found to meet training needs. Nonetheless, the impact of training was limited in magnitude and duration.

Conclusion: Improvement of ADR reporting activity requires a multidisciplinary approach incorporating the use of team champions, publicity campaigns, more efficient use of technology to enhance the reporting experience, periodical tailored training and feedback.

Further sources of information/References:

1. Stewart D, et al. Non-medical prescribers and pharmacovigilance: participation, competence and future needs. Int J Clin Pharm 2013;35:268 -74

\section{The role of Pharmacovigilance in the development of Pharmacogenetics treatments}

\author{
E. Arslan Unlu ${ }^{1}$, B. Unal ${ }^{1}$ \\ ${ }^{1}$ Bayer Turk Kim. San. Ltd. Sti., Istanbul, Turkey
}

Background: Various types of drugs are available for the treatment of a wide spectrum of disorders. In medicine, differences among people regarding the drug responsiveness, tolerance, sensitiveness to a particular drug are obviously one of the important problems in today's world Genetic and race/ethnicity differences among patients may contribute to differences in medication response, as well as the development of adverse effects. Some individuals may represent more predispositions to certain ADRs (Adverse Drug Reactions) via genetic polymorphisms that change their responses to the specific drugs. According to scientific studies, it has been proved that the individual variations in the genetic polymorphisms have an impact on personalized medical treatments. Genetic variations have an effect on the enzymes that enable drugs to be metabolized and removed from the body and it has reduced or increased their activity. As pharmacogenetics is a term that refers to the use of molecular genetic approaches to understand differences in drug response and tolerability, this article wants to show the role of pharmacovigilance in the development of pharmacogenetics treatments.

Objective: The article aims to link pharmacovigilance with pharmacogenetics in order to describe the personalized treatments according to genetic factors and to underlie the importance of adverse event reporting for the development of tailored drug treatments. 
Method: Under this respect, pharmacogenetics treatments and their contributions to ADRs were reviewed in accordance with Pharmacovigilance processes.

Results: ADR reporting is indispensable for monitoring important safety issue and signal detection in the aforesaid therapy. Assessing and interpreting these reports can lead to changes in clinical assessment and medical management. Scientific data show that several drug-related adverse reactions can result in the standard drug treatment. There can also be considerable morbidity, particularly with drug-induced diseases. Therefore, individualized medical treatment to minimize the incidence of adverse drug reactions is a need in drug management and has improved to combine individual genomic information into a patient's clinical assessment and family history. The researchers believe that the pharmacogenetics therapy is safer and more effective than the standard drug therapy. Furthermore, to reduce side effects, they expect that pharmacogenetics treatments for some diseases will become the standard of care.

Conclusion: The construal of ADR reports is significant in the development of pharmacogenetics treatments. Thus, it is important to continue to monitor closely pharmacogenetics therapy in clinical practice to improve the knowledge on their safety and efficiency. Implementation of this therapy will be one of the cornerstones of personalized medicine.

Further sources of information/References:

1. Bondon-GuittonE, et al. The contribution of pharmacogenetics to pharmacovigilance. Therapie 2016; 71: 223-28

2. Howland RH. Pharmacogenetics and Pharmacovigilance. Drug Saf 2009; 32: 265-270

3. Nelson MR, et al. Genome-wide approaches to identify pharmacogenetic contributions to adverse drug reactions. Pharmacogenomics $\mathrm{J}$ 2009; 9:23-3

\section{Peripheral neuropathy and nail's blue coloration under folfiri-bevacizumab}

K. Lechheb ${ }^{1}$, I. Bennani ${ }^{1}$, A. Tebaa ${ }^{2}$, A.1 Mrani Alaoui ${ }^{1}$, O. Ziraoui $^{1}$, Y. Tadlaoui ${ }^{1}$, Y. Bousliman ${ }^{1}$

${ }^{1}$ Pharmacy Departement Hopital Militaire Mohammed V, Rabat, Morocco, ${ }^{2}$ Centre d'antipoison et Pharmacovigilance, Rabat, Morocco

Background: The side effects of chemotherapy vary depending on the drugs used, Dosages and people. Each responds differently to treatments. However, if they become too large or if the patient does not support any of the drugs used, the treatment may be altered or interrupted to allow the body to recover.

Objective: The objective of this work is to determine the accountability in a patient with peripheral neuropathy and a nail's blue coloration under Folfiri-bevacizumab

Methods: We report a case of a 64 -year-old patient, $70 \mathrm{~kg}$, followed by metastatic colon cancer and after the 3rd cure of Foliri (5fluorouracil and Irinotecan) -bevacizumab peripheral neuropathy and nail's blue coloration.

To determine the accountability of these drugs, we used the French method of accountability.

Results:

The delay of neuropathy was compatible for 3 drugs

The evolution of the three drugs was suggestive.
The rechallenge was 0

On the data of the literature, the peripheral neuropathy is a notorious effect already described for 5 fluorouracil and bevacizumab

The delay of the occurrence of the nail's blue coloration was compatible for the 3 drugs

The evolution was not suggestive

The rechallenge was 0

Conclusion:

The accountability score for peripheral neuropathy was:

I5B4 for 5fluorouracil

I5B4 for bevacizumab

I5B3 for irinotecan

The accountability score for nail's blue coloration was

I3B3 for 5fluorouracil

I4B3 for bevacizumab

I2B1 for irinotecan

The level of informativity for the three molecules is 2 .

In our patient, it was therefore tempting to retain bevacizumab and 5fluorouracil as responsible for peripheral neuropathy and the coloring of the nails in blue.

\section{Medication errors in real clinical practice- observational study among women undergoing assisted reproduction therapy}

\author{
S. Stoev ${ }^{1}$, H. Lebanova ${ }^{1}$, I. Getov ${ }^{1}$ \\ ${ }^{1}$ Medical University Sofia, Sofia, Bulgaria
}

Objective: To identify the type, frequency and preventability of drugrelated problems (DRP) from pharmacovigilance perspective. To evaluate the role of the pharmacist for mining and prevention of medication errors and adverse drug reactions (ADRs) in the therapeutic process.

Methods: Prospective clinical observation, performed at a specialized obstetric/gynecology clinic by three indipendent observers with pharmaceutical education for the period from 03.01.2015 to 01.04.2016.

Results: The total number of indicated DRPs was 67. Observers evaluated 44 of the detected cases as ADRs. 23 of the adverse effects were clinically significant, while only $11 \%$ of the observed ADRs led to discontinuation of the product or any complication leading to hospitalization or prolongation of hospitalization. DRP are associated mainly with drug misuse, wrong medication prescribed, overdosing or too long course of treatment. Medication errors were identified in the process of prescribing or managing the prescribing information both in hospital electronic database or paper prescription forms. Only $12 \%$ of cases were registered by obervers as a result of a physician request for a clinical pharmacy consult. The accpetance level of phisycans with the proposed corrective actions was more than $80 \%$.

An analysis of several clinical cases, demonstrating serious complications as a result of a preventable ADR, has been performed.

Conclusion: Establishment of a system for constant drug use monitoring and active involvement of pharmacists in the therapeutic approach management would guarantee earlier detection and reduction of preventable ADRs and would optimize the treatment process. 
257 The Chester Experience-The impact of an intervention to improve Adverse Drug Reactions reporting in a district general hospital

\author{
$\underline{\text { A. } \operatorname{Abbas}^{1}}$, C. Green ${ }^{1}$ \\ ${ }^{I}$ Countess of Chester Hospital NHS Foundation Trust, Chester, UK
}

Background: Under-reporting of Adverse Drug Reactions (ADRs) is a major public health concern globally and is a major limitation of spontaneous reporting systems. The Yellow Card Scheme (YCS) is the UK's principal system for reporting ADRs. Only $6-10 \%$ of serious ADRs are reported through the YCS. Under-reporting of ADRs by healthcare professionals is a key problem [1]. It is estimated that $6.5 \%$ of all acute hospital admissions in the UK are related to an ADR [2]. Baseline ADR reporting rate at the Countess of Chester Hospital $(\mathrm{CoCH})$, a 600 beds district general hospital in England was negligible in 2012-2013 but improved dramatically after implementing a plan in January-2014.

Aims: To illustrate the methods used to improve ADR reporting at $\mathrm{CoCH}$. Methods: A target of 5 ADR reports / week was set by the pharmacy team. Teams initially focused on admission areas and medical specialities (rheumatology, gastroenterology and haematology) where drugs of special interest to the Medicines \& Healthcare Products Regulatory Agency (MHRA) [3] (biologics, biosimilars and novel oral anticoagulants) are used. An individual monthly reporting target of a minimum of 1 ADR report per pharmacist was set. A campaign to highlight ADRs at ward rounds and train pharmacists, doctors and non-medical nurse prescribers in pharmacovigilance was implemented. Reporting performance was received from the MHRA via the Yellow Card Centre North West (YCCNW) who oversees reporting from 31 acute and community trusts and produces annual league tables.

Results: Table 1: ADR reporting performance at $\mathrm{CoCH}$ over the last 5 years

\begin{tabular}{|l|l|l|l|}
\hline Year & Total & Reports by Pharmacy & $\begin{array}{l}\text { YCCNW league } \\
\text { standing }\end{array}$ \\
\hline $2012-13$ & 17 & 4 & $18^{\text {th }}$ \\
\hline $2013-14$ & 68 & 62 & $3^{\text {rd }}$ \\
\hline $2014-15$ & 207 & 193 & $1^{\text {st }}$ \\
\hline $2015-16$ & 416 & 389 & $2^{\text {nd }}$ \\
\hline $2016-17$ & 384 & 343 & $1^{\text {st }}$ \\
\hline
\end{tabular}

A 22 fold increase in total reporting in comparison to baseline was observed. Most significant effects were observed within pharmacy where individual reporting targets were set and monitored.

Conclusion: Improvement of ADR reporting activity requires a multidisciplinary approach including education and publicity campaigns but equally importantly setting targets, monitoring and feeding back to reporters.

\section{Further sources of information/References}

1. Stewart D, et al. Non-medical prescribers and pharmacovigilance: participation, competence and future needs. Int J Clin Pharm 2013; 35: $268-74$

2. Pirmohamed M, James S, Meakin S, Green C, Andrew K. Adverse Reactions as a cause of admissions to hospital: prospective analysis of 18820 patients. BMJ 2004; 329: 15-9

3. MHRA. Yellowcard: helping to make medicines safer. [cited May 2017] http://yellowcard.mhra.gov.uk
261 Evaluation of the antimicrobial effect of expired oral antibiotics

\author{
$\underline{\text { K. Alghasham }}{ }^{1}$ \\ ${ }^{1}$ Pharmacist, Almubarraz, Saudi Arabia
}

Introduction: In spite of significant risks, as well as non-clinical importance due to loss of potency, stiff penalties against administration of expired antibiotics are still not appropriately enforced by health policy makers in many developing countries, possibly because of little evidence to support that expired medications are hazardous

Objective: To investigate the effect of expiration dates on in vitro bacteriostatic potentials of different oral antibiotics. The potency of five antibiotics, both expired and unexpired, Azithromycin,Clarithromycin, Levofloxacin and Cloxicillin, and tetracycline were investigated, based on the antimicrobial potentials. The investigation was done by challenging local isolates of different concentrations of $S$. aureus bacteria; using Disc diffusion technique.

Results and Discussion: Although the expired antibiotics potency was less than that of the control ones, it was found that the microorganisms challenged with these antibiotics were evaluated as susceptible (According to NCCLS), as the diameter of inhibition zone lies within the susceptible range

\section{Recommendations:}

1. If the antibiotic is needed, and the patient is not able to replace the expired antibiotic, there is no evidence that it is unsafe to take the medication in most cases

2. Guidelines for storage of essential medicine should be followed with antibiotics.

3. Public health policies based on national guidelines should include monitoring of the quality control of the expired antibiotic

Further sources of information/References:

1. Marshall BM, Ochieng DJ, Levy SB. Commensals: unappreciated reservoir of antibiotic resistance. Microbe 2009; 4:231-8

2. Alekshun MN,Levy SB. Molecular mechanisms of antibacterial multidrug resistance. Cell 2007; 128:1037-50

3. Lipsitch M. Fears growing over bacteria resistant to expired antibiotics. New York Times Sep.2012 [Pleaes provide more information, such as URL]

4. Chen DW, Chang Y, Hsieh PH, Ueng SW, Lee MS. The influence of storage temperature on the antibiotic release of vancomycin-loaded polymethylmethacrylate. ScientificWorldJournal 2013; 2013: 573526

\section{Estimating the Pertinent Information Present in Social Media, not just what an Algorithm Detects}

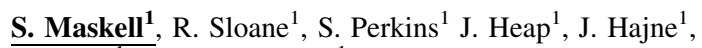
A. Jones ${ }^{1}$, M. Pirmohamed ${ }^{1}$

\section{${ }^{1}$ University of Liverpool, Liverpool, $U K$}

Background: Social media has great potential as a source of information pertinent to pharmacovigilance. Algorithms have been developed to extract this information. Previous studies have characterised the performance of these algorithms and investigated the potential utility of the information extracted relative to that stored in spontaneous reporting databases. However, these studies each consider different algorithms. As a result, it is unclear what information could ever be extracted from a fixed 
social media dataset. This makes it challenging to quantify the utility of social media independently of the algorithms used to process the data.

Objective/Aim: This objective of this research is to develop a method for estimating the information pertinent to pharmacovigilance that could ever be extracted from a social media dataset.

Methods: As in previous studies, we characterise the performance of an algorithm by considering some manually annotated social media posts. We stratify our analysis by considering each drug and adverse event in turn as a condition. For each stratum, the annotations then indicate whether a post is condition positive or condition negative. We assume that an algorithm, when applied to the same annotated post, either generates a detection or does not. In contrast to previous studies, we observe that it is possible to use the annotations and detections to estimate the ratio of the number of condition positive posts to the total number of detections. We call this ratio the "inverse yield". Crucially, an algorithm's inverse yield can be used to estimate the number of condition positive posts present from the number of detections generated when the algorithm is applied to some other (assumed unannotated) data.

Results: The dataset considered involved posts taken from Twitter and Facebook over a three year period. A specific processing chain was considered in the context of 38 products and over 1000 preferred terms. A characterisation of tens of thousands of manually annotated data, stratified across products and preferred terms, was considered. The inverse yield, as calculated using these annotated data and the associated algorithmic outputs, highlighted that the variation across preferred terms was significant when considering the relative utility of social media and spontaneous reporting.

Conclusion: A method has been developed that enables quantities calculated when characterising the performance of social media processing algorithms to be used to estimate the information present in the data feeding such algorithms.

\section{Looking Longitudinally in Twitter: Reading More than 140 Characters}

\author{
S. Maskell ${ }^{1}$, R. Sloane ${ }^{1}$, J. Hajne ${ }^{1}$, J. Heap ${ }^{1}$, S. Perkins ${ }^{1}$, \\ E. Griffith $^{1}$, A. Jones ${ }^{1}$, M. Pirmohamed ${ }^{1}$ \\ ${ }^{1}$ University of Liverpool, Liverpool, $U K$
}

Background: Each Tweet is only 140 characters long. It is therefore highly unlikely that individual Tweets contain text describing more than a single drug as well as text describing an adverse event. It is also highly unlikely that Tweets contain information about taking a drug at one time and experiencing an adverse event at a (very) different time. However, it is possible to extract the history of Tweets that a Twitter user has posted. While much of a user's history will be irrelevant to drug safety, the longitudinal history has the potential to contain more information pertinent to pharmacovigilance than individual Tweets. However, this potential is not currently quantified.

Objective/Aim: This study aims to quantify the relative value of individual Tweets and the histories of Tweets in three specific contexts: interactions between drugs and alcohol; interactions between drugs and grapefruit juice; adverse events related to pregnancy.

Methods: Given that some drugs had been identified as positive and negative controls, in the context of each drug, an automated processing chain was used to identify Tweets that mentioned the drug and an adverse event. The histories of Tweets posted by the same users were extracted. The same automated processing chain as considered previously was then applied to the posts comprising these longitudinal histories. Given the outputs from processing individual Tweets and from processing longitudinal histories, the relative performance in the three contexts considered was characterised. This characterisation was performed in terms of the ability to detect signals relevant to the contexts considered.

Results: A total of six drugs were each identified with each being either a positive or negative controls with respect to at least one of the three contexts considered. Data from Twitter collected over a three year period was considered and processed with the aim of detecting the presence of over 1000 preferred terms. Each longitudinal history consisted of a maximum of 3000 posts and typically spanned 18 months. The relative performance in the three contexts considered was characterised.

Conclusion: It is possible to extract a Twitter user's history and so extract information pertinent to drug safety that cannot be extracted from individual Tweets. There is potential for such longitudinal histories to enable social media to provide information that complements that which is only infrequently encountered in spontaneous reporting databases.

\section{Strengthening Collaborations for Operating Pharmacovigilance in Europe (SCOPE) Joint Action: supporting medicines regulators in EU to improve public health}

\author{
$\underline{\text { L. Loughlin }^{1},}$ A. Radecka ${ }^{1}$ \\ ${ }^{1}$ SCOPE Joint Action, London, UK
}

Background: In November 2013, a European team of regulators initiated the Strengthening Collaboration for Operating Pharmacovigilance in Europe (SCOPE) Joint Action to identify and address key areas relevant to supporting operation of pharmacovigilance legislation that came into effect in June 2012. Funded by the by the Health Programme of the European Union, and with contributions from the involved member states, SCOPE gathered information and expertise on how regulators in member states run their national pharmacovigilance systems and from the findings developed guidance and other training materials.

Objective/Aim: There were a number of aims of the SCOPE Joint Action:

- Enable Member States to gain a fuller understanding of, and develop best practice in reporting mechanisms for adverse drug reactions

- Create a shared understanding and implementation of best practice in signal management across the EU network

- Help the achieve good practice in Risk Communications

- Enable Member States to understand, and develop their quality management systems for pharmacovigilance

- Develop competencies to support pharmacovigilance throughout the product lifecycle

- Strengthen regulatory collaboration

Methods: SCOPE gathered information and expertise on how regulators in member states run their national pharmacovigilance systems. The project was divided into eight separate work streams. Five of these concentrated-on pharmacovigilance topics-collecting information on adverse drug reactions, identifying safety issues, communicating risk and assessing risk minimisation measures, supported by effective quality management systems. The other three focused on the functional aspectscoordination, communication and evaluation. From the findings of the five pharmacovigilance topic areas, guidance and other training materials were developed and workshops and training events were held.

Results: SCOPE delivered guidance and training in key aspects of pharmacovigilance and developed tools and templates to support good practice that those working in the medicines regulatory agencies can use to 
strengthen their national systems. The materials are available at www.scopejointaction.eu and are also being made available through the EU Network Training Centre.

Conclusions: Regulators have worked together, and in partnership with others, to improve the skills and capacity in the pharmacovigilance network to help safeguard public health in both national territories and the EU as a whole. The Joint Action supports consistent pharmacovigilance operations approaches throughout the EU network, benefiting the safety monitoring of medicines and communication outputs, thereby helping to safeguard public health.

\section{Checkpoint Inhibitor induced thyroid immune related adverse events: two distinct clinical patterns}

\author{
A. Olsson-Brown' 1 , R. Lord ${ }^{2}$, J. Sacco ${ }^{1,2}$, J. Upton ${ }^{2}$, M. Coles ${ }^{3}$, \\ M. Pirmohamed ${ }^{1}$ \\ ${ }^{1}$ University $f$ Liverpool, Liverpool, $U K,{ }^{2}$ The Clatterbridge Cancer \\ Centre, Wirral, UK, ${ }^{3}$ University of York, York, UK
}

Background: Immune checkpoint inhibitors (CPI) are used in the mainstream treatment of a rapidly increasing number of malignancies. CPIs have novel toxicities compared to those recognised within oncology. These toxicities are immune related adverse events (irAEs) and clinically mimic autoimmune disease. One such irAE is thyroid dysfunction which, whilst rarely seen with the CTLA-4 inhibitor ipilimumab, has an incidence of $6-11 \%$ PD-1 inhibitors $[1,2,3]$. Thyroid irAEs have a significant clinical impact with permeant impairment, generally requiring long-term replacement; notable clinical, often multidisciplinary, input to stabilise endocrine function and frequently delays in oncological therapy [4].

Aims/ Objectives: This study evaluated the clinical distribution of thyroid irAEs to determine hallmarks patterns specific to CPI thyroiditis Methods; A retrospective review of all patients treated with PD-1 CPI for metastatic melanoma treated at the Clatterbridge Cancer Centre over a 16-month period was undertaken identifying patients with thyroid irAEs. The clinical course and emerging clinical patterns of toxicity were evaluated to determine the presence of specific clinical trajectories within the patient cohort.

Results: Between February 2016 and May 201789 patients with metastatic melanoma were treated with monotherapy PD-1 CPI, 12 (13.5\%) of whom developed thyroid dysfunction. There were two distinct patterns identified: a hyperthyroid phase followed by a hypothyroid phase and de novo hypothyroidism. No additional patterns were identified. The majority of patients $(66 \%)$ illustrated a hyper/hypo pattern where a period of hyperthyroidism was identified with peak T4 38.94 (23.1-52.0) and trough TSH $<0.1$. This pattern occurred following cycle 3 (range 2-8) of treatment. A subsequent decline in T4 followed within 6-21 days before hypothyroidism was identified in 8.5 weeks (range 3-10 weeks) from irAE commencement. Thirty-three percent of patients displayed de novo hypothyroidism. All patients were female and the irAE occurred after cycle 4 (range 2-6). The trough T4 was 4.15 (1-5.6) and the peak TSH 68.54 (51.26-79.35). All patients displayed minor symptoms inadequate to be considered clinical thyroid dysfunction. Steroids provided little benefit and the only pharmaceutical therapy required was levothyroxine. Symptomatic management for hyperthyroidism was not required by any patient within this series. All patients had permanent dysfunction requiring replacement. All continued with immunotherapy treatment but there were significant treatment hiatuses for toxicity, the longest of which was 5 months.

Conclusions: This retrospective review has characterised the manifestation of thyroid irAE following CPI monotherapy which informs understanding and clinical management in terms particularly in terms of pattern, phase duration and pharmacological management.

Further sources of information/References:

1. Larkin J, Chiarion-Sileni V, Gonzalez JJ, et al. Combined Nivolumab and Ipilimumab or Monotherapy in Untreated Melanoma. New Engl J Med 2015; 373:23-34

2. Robert C, Schachter J, Long G, et al. Pembrolizumab versus Ipilimumab in Advanced Melanoma. New Engl J Med 2015; 372: 2521-32

3. Hodi FS, O'Day SJ, McDermott DF, et al. Improved survival with ipilimumab in patients with metastatic melanoma. New Engl J Med 363: 711-723

4. Topliss DJ. Clinical update in aspects of the management of autoimmune thyroid diseases,. Endocrinol Metab 2016; 31:493-9

\section{Disposal of Unused and Expired Medicines by Consumers-implications for Ecopharmacovigilance}

\author{
Y. Yirenkyiwaa Esseku ${ }^{\mathbf{1 , 2 , 3 , 4}}$, E. Woode ${ }^{4}$, A. Nii Oto Dodoo ${ }^{2,3}$
}

${ }^{1}$ Rapha Consult, Accra, Ghana, ${ }^{2}$ African Collaborating Centre for Pharmacovigilance, Accra, Ghana, ${ }^{3} \mathrm{WHO}$ Collaborating Centre for Advocacy and Training in Pharmacovigilance, Accra, Ghana, ${ }^{4}$ Kwame Nkrumah University of Science and Technology, Kumasi, Ghana

Introduction: Medicines are used extensively across the world on daily basis for varied purposes and different periods of time. After acquisition, some medicines end up unused or expired while in the possession of consumers who must find ways of disposing of them [1]. The methods of disposal may result in some environmental effects as a direct result of the presence of pharmaceuticals in the environment. Inadvertent exposure to animals and plants may also result in unintended effects on exposed organisms [2].

Aim: To identify the patterns of disposal methods used by consumers when they no longer have use of the products and assess the consequences of these disposal patterns for Ecopharmacovigilance.

Methods: Questionnaires were administered to consumers to gather information on the ways in which they dispose of their unused and expired medicines. The information gathered was analysed to assess the patterns of disposal used by consumers in the disposal of unused or expired medicines. Results: The study shows that up to $51 \%$ of consumers have unused or expired medicines from a visit to a health facility or had medicines stored up for emergency. These medicines, if unused or expired, must be disposed of by the consumers. The study further showed that over $70 \%$ of respondents dispose of medicines in ways to introduce the pharmaceutical and personal care products (PPCPs) directly into environmental media. Other available forms of disposal ultimately deposit the medicines into environmental media as there are no measures in place for ensuring the protection of environmental media from exposure.

Conclusion: The methods used by consumers for the disposal of unused and expired medicines are likely to result in varied effects on the environment and exposed plants and animals. There are no requirements for disposal to be undertaken in a manner that protects environmental media or exposed animals and plants. There is the need for the introduction of national laws aimed at reducing the introduction of medicines into environmental media.

\section{Further sources of information/References}

1. Esseku YY, Esseku, H. Disposal of medicines and impact on water sources. Sustaining Water and Sanitation Services for all in a Fast 
Changing World. $37^{\text {th }}$ WEDC International Conference, Hanoi, Vietnam, 2014. Available from http://wedc.lboro.ac.uk/ resources/conference/37/Esseku-2028.pdf

2. Esseku YY. Ecopharmacovigilance in Practice: Design of an intervention-the Drug Disposal Flow Diagram. 2016 (Unpublished). Available from http://ir.knust.edu.gh/xmlui/handle/123456789/9354

\section{Phenotypic Approaches to Risk Management}

\author{
K. Arnold Chan \\ University of Harvard, Boston, USA
}

The term "risk management" was introduced to modern medicine and medical product regulation some years ago by regulatory agencies, but the concept should not be new to the clinical community, who would not want to cause any harm to patients. The term "Therapeutic Risk Management" is preferred by some organizations, with the objectives of "maximizing benefit and minimizing risk" when medical products are used in day-today real world settings.

In this presentation a brief history of therapeutic risk management programs for drugs will be reviewed, including the early examples of isotretinoin and clozapine. Subsequent evolvement of regulatory guidelines and practices associated with therapeutic risk management will be described and illustrated with examples.

The last part of the presentation will highlight the importance of evaluating the effectiveness of therapeutic risk management programs, including 'process metrics' and 'outcome metrics.' From a regulatory perspective, it is not uncommon for regulatory agencies to require some level of risk management activities (for example, risk evaluation and minimization strategy [REMS] in the USA), without prior evidence to support the use of those strategies. Moreover, the effectiveness of the programs may not be systematically evaluated or evaluated in a valid manner. Most of the time the evaluation focused on the implementation, rather than outcomes, of the therapeutic risk management programs. Examples of such "process metrics" include whether educational messages reached the target health care providers or whether certain laboratory tests were ordered according to the therapeutic risk management program. While those are important, the more important objective should be to reduce adverse outcomes. Methods and challenges related to measuring relevant outcomes of therapeutic risk management programs will be discussed.

\section{Pharmacogenomics and prevention of serious cutaneous adverse drug reactions in Clinical Practice in Thailand}

\section{Sukasem ${ }^{1}$}

${ }^{I}$ Department of Pathology, Faculty of Medicine Ramathibodi Hospital, Mahidol University, Thailand

Focus screening of prevalent risk alleles; HLA $B * 15: 02, H L A-B * 58: 01$ and $H L A-B * 57: 01$, before high risk populations starting carbamazepine, allopurinol and abacavir therapy, respectively. Can it be significantly prevented occurrence of severe cutaneous adverse drug reactions (SCARs) globally? Currently, several guidelines recommend genetic tests for various $H L A-B$ alleles and dosages of drugs that induce SCARs among specific patients carrying risk alleles. With evidence linking association, the Clinical Pharmacogenetics Implementation Consortium (CPIC) dosing guidelines recommend the use of pharmacogenomic tests for presence of the markers before initiating drug therapy in patients. Individuals carrying one or two copies of these markers during clinical genotyping tests presented as "positive", thus implying a high risk of drug induced- SCARs. Therefore, the drug is not recommended in such patients, as there is high risk of drug-induced SCARs. When there are no copies of the allele detected in genotype tests, a "negative" result is recorded. Patients with negative tests can use the particular drug, according to standard dosing guidelines, because of their lower risk of SCARs related to the use of the drug. The distinct pharmacogenomics of SCARs arise from the varied frequencies of HLA alleles and clinical phenotypes in different ethnic groups. A majority of HLA alleles associated with SCARs are highly prevalent in Southeast Asian populations. In Thailand, the Thai universal health coverage scheme has done the screening program since 2014, reimbursing the cost of pharmacogenetic testing about US\$28 per patient for carbamazepine treatment. Therefore, the incidence of carbamazepineinduced SJS/TEN has been reduced sharply and the country is now working to eradicate this life-threatening adverse events. To strengthen the utilization of pharmacogenetic testing in Thailand, we have first invented a low-tech approach and launched "a pharmacogenetic wallet card". After patients have taken the pharmacogenetic test, their results are entered into the "Pharmacogenetic ID card," a purple rectangle and plastic wallet size that they can carry around and show to future doctors with their genomic results of those related to the risk of adverse drug reactions or dose recommendation. For example, after patients have taken an HLA test, their results are entered into a plastic "pharmacogenomic wallet card", which basically contains the genomic results of those related to the risk of SJS/ TEN. This card can be carried around and shown to different doctors in the future.

\section{Social Media and Pharmacovigilance (an Industry Perspective)}

\section{Lewis ${ }^{1}$}

\section{Novartis, UK United KingdomUK}

Pharmacovigilance specialists across the pharmaceutical industry are wary about social media and the potential for sharing of diverse, even spurious, data relating to adverse effects. Urban myths have created concern about large volumes of reports gleaned from crawling social media looking for adverse reactions to authorized medicines. Discussions have taken place on the role of the pharmaceutical industry, focusing on the need to collect and report adverse reactions from this diverse source of data. As recently as 2011, it was declared by an expert group that this was considered an “...impossible task..." [1]. The IMI Web-RADR consortium [2] was formed in 2014 with the aim of examining the pharmacovigilance of social media. Early in the life of the project, a hypothesis was generated that " $\mathrm{A}$ key benefit of social media is that it can be mined to generate hypotheses related to drug efficacy and safety." [3] . It was considered possible that social media sources may provide data for the detection of safety concerns in real time. Many challenges were recognised, including the posting of misinformation, either deliberately or accidentally, difficulties in the detection of adverse events due to the large volume of data, the uncontrolled nature of the content, and the potential concern of pharmaceutical companies being viewed as 'Big Brother' by users of social media [4]. Research into the pharmacovigilance of social media has been conducted by Web-RADR (involving regulatory authorities, patients, academic centres, non-government associations, and the pharmaceutical industry 
[5]). One of the aims of the research was to compare traditional methods of safety surveillance, such as spontaneous reporting, with the results of careful scrutiny of social media. The research was performed to appropriate standards for pharmacovigilance research, with an emphasis on the protection of data privacy. Data and information will be presented on the process, tools and techniques developed, content of social media sites available for research, and the results of the experiments conducted. Studies completed to date suggest that information on social media provides a potential resource for data mining, but there are caveats. Evidence is emerging that patient reported safety outcomes are content-rich with descriptions of symptoms, but diagnostic data are lacking by comparison. This is consistent with recent findings on patient-reported adverse reactions submitted via spontaneous reporting systems [6]. Recommendations concerning good pharmacovigilance practices are being prepared based on the learnings derived from Web-RADR and these will be published.

Further sources of information/References:

1. Edwards IR,Lindquist M. Social Media and Networks in Pharmacovigilance Boon or Bane? Drug Saf 2011; 34: 267-71

2. Web-RADR: Recognising Adverse Drug Reactions. Accessed @ https://web-radr.eu/ on 16 June 2017

3. Naik P, Umrath T, van Stekelenborg J, Ruben R, Abdul-Karim N, Boland R, et al. Regulatory Definitions and Good Pharmacovigilance Practices in Social Media: Challenges and Recommendations. Ther Innov Regul Sci 2015; 49:840-51

4. Sukkar E. Searching Social Networks to Detect Adverse Reactions. Pharm J 2015; 294: 75-8

5. Sloan R, Osanlou O, Lewis D, Bollegala D, Maskell S, Pirmohamed M. Social Media and Pharmacovigilance: A Review of the Opportunities and Challenges. Br J Clin Pharmacol 2015; 80 : 910-20

6. Banovac M, Candore G, Slattery J, Hoü̈ez F, Haerry D, Genov G, Arlett P. Patient Reporting in the EU: Analysis of EudraVigilance Data. Drug Saf 2017; 40: 629-45

\section{Pharmacovigilance of biologics and biosimilars}

\section{G. Trifiro}

Department of Biomedical and Dental Sciences and Morphofunctional Imaging, University of Messina, Italy \& Department of Medical Informatics-Erasmus Medical CenterRotterdam, the Netherlands

Highly innovative and costly biologics improved dramatically the management of high-burden diseases such as autoimmune diseases (e.g. TNFalfa antagonists), cancers (e.g. rituximab, trastuzumab), and chronic renal failure (e.g. epoetins) [1]. Specific serious safety issues such as administration site adverse reactions, infections and tumours, however are more likely to occur with biologics than with non-biologic drugs [2].

In the last decade, several widely prescribed biologics lost the patent thus allowing the marketing of biosimilars which are approved by regulatory agencies on the basis of documented comparability of efficacy, safety and quality with respect to corresponding originator. The real benefit-risk profile of biosimilars however has been questioned due to limited amount of premarketing information. Nevertheless, after more than ten years of marketing from the first biosimilar no proof of differences in the safety profile of biosimilars and originators has been reported.

In the past, batch-specific safety issues due to differences in the manufacturing process have been described. For this reason, another relevant issue concerning pharmacovigilance of biologics/biosimilars is the ability to trace specific batch administered to the patient in case an adverse reactions occurs, as highlighted in the EU pharmacovigilance legislation. Only around $20-25 \%$ of the spontaneously reported adverse events for biologics to the EU Eudravigilance database and the US FDA adverse event reporting system contain information on the batch number, respectively [3].

In addition, in clinical practice up to $20 \%$ frequency of switch (also reverse and multiple switches) between different biologics/biosimilars in various therapeutic areas during the first year of therapy has been reported. This common practice may limit the correct causality assessment of the ADRs occurring during biologics/biosimilars treatment. On the other side, it has been hypothesized that switching between different biologics may be associated to increased risk of immunogenicity, concern that has not been confirmed so far in a large number of both randomized clinical trials and observational studies

With the advent of a growing number of innovative biologics on a side and biosimilars in other therapeutic areas as oncology on the other side, there is a need to put in place a more efficient post-marketing surveillance system of these drugs, which may profit of availability of large amount of electronic healthcare databases which may complement spontaneous reporting system.

Further sources of information/References:

1. Biologics and the Principles of Health Insurance What is the purpose of health insurance? This review of basic insurance concepts provides a context for discussions about coverage of biologics. Biotechnol Healthc. 2012; 9(2): 14-8

2. Cutroneo PM, et al. Safety Profile of Biological Medicines as Compared with Non-Biologicals: An Analysis of the Italian Spontaneous Reporting System Database. Drug Saf 2014; 37:961-70

3. Vermeer NS, Straus SMJM, Mantel-Teeuwisse AK, Domergue F, Egberts TCG, Leufkens HGM, De Bruin ML. Traceability of biopharmaceuticals in spontaneous reporting systems: a cross sectional study in the FDA Adverse Event Reporting System (FAERS) and EudraVigilance Databases. Drug Saf 2013;36:617-25

\section{Pre-emptive genotyping for preventing ADRs}

\section{H. Guchelaar ${ }^{1}$}

${ }^{1}$ Leiden University Medical Centre, Leiden, the Netherlands

Pharmacogenomics (PGx), the study of genetic variability affecting an individual's response to a drug, holds the promise to lead to more efficacious, safer and cost-effective drug therapy. In recent years, guidelines with therapeutic recommendations have become available to guide clinicians how to interpret and adjust drug therapy based upon a pharmacogenomic test result. Indeed, for some drug-gene pairs (such as capecitabine-DPYD; mercaptopurine-TPMT) pre-therapeutic testing has been implemented in routine clinical care but their remain barriers for widescale implementation of pre-therapeutic testing.

Studies have shown that at least $90-95 \%$ of patients have an actionable genotype when tested for a panel of pharmacogenes including CYP2C9, CYP2C19, CYP2D6, CYP3A5, DYPD, SLCO1B1, TPMT, HLA-B, UGT1A1 and VKORC1. Therefore, pre-emptive testing (that is: multiple pharmacogenomic variants are collected prospectively and embedded into the patient's medical record) of a panel of genetic variants seems a promising and cost-effective approach.

We have performed a pilot study in 200 primary care patients with preemptive testing of a PGx panel with the aim to reduce ADRs. In addition, the EU Horizon2020 funded U-PGx consortium was established and investigates a pre-emptive genotyping approach of a panel of important 
pharmacogenomic variants as a new model of personalised medicine. To meet this goal, 84 existing pharmacogenomics guidelines of the Dutch Pharmacogenomics Working Party of the Royal Pharmacists Association (KNMP) are combined with novel IT solutions. Implementation is conducted at a large scale in seven European health care environments (The Netherlands, UK, Spain, Italy, Austria, Greece and Slovenia) for a total of 8100 patients and accounts fort he diversity in health system organisations and settings. The mulicenter randomized study is open since Q1 2017. Feasibility, health outcome and cost-effectiveness are investigated; the study is powered to show a reduction of $30 \%$ of the incidence of grade 2 or higher adverse drug events.

The U-PGx consortium (www.upgx.eu) ultimately aims to formulate European strategies for improving clinical implementation of pharmacogenomics.

\section{The Bengt Erik Wiholm Lecture 2017: The Language of Pharmacovigilance}

\section{$\underline{\text { J.K Aronson }}{ }^{1}$}

\section{Unviersity of Oxford, Oxford, UK}

Thesis: I take it as axiomatic that the words we use and the ways in which we use them are important. Thus, how we communicate information is crucial to the proper practice of pharmacovigilance, and an appreciation of the linguistic problems of so doing is essential. As John Locke wrote, in An Essay Concerning Human Understanding (1690), "He that uses his words loosely and unsteadily will either not be minded or not understood." I have therefore chosen as my subject for the 2017 Bengt Erik Wiholm Lecture "The Language of Pharmacovigilance".

Antithesis: In A Dictionary of Modern English Usage (Oxford, 1926), Henry W Fowler described the words pleistocene, pliocene, and miocene as "regrettable BARBARISMS", and added "It is worth while to mention this, not because the words themselves can now be either mended or ended, but on the chance that the men of science may some day wake up to their duties to the language - duties much less simple than they are apt to suppose."

Much later, the erstwhile Wykeham Professor of Logic in Oxford, Michael Dummett, in a book called Grammar and Style (Duckworth, 1993), which is good on style but too prescriptive on grammar for modern taste, wrote about style that "our object should be to make our writing unobstrusive, so that its sense can be immediately apprehended and the reader is never distracted from it."

These two observations remind us of two important things about the language we use, but do not perhaps sufficiently emphasize the attendant difficulties, especially for those for whom English is not their first language, and even sometimes those for whom it is.

Ludwig Wittgenstein (Philosophical Investigations, §43) suggested that "For a large class of cases ... the meaning of a word is its use in the language [Die Bedeutung eines Wortes ist sein Gebrauch in der Sprache]", implying that words mean what we determine and agree them to mean, through the ways in which we use them. That tenet works well in general conversation, but it is not enough for experts in specialist areas to simply agree about what the words mean; the words must also be comprehensible to those who are not expert. This potentially creates problems when the words used are technical.

Synthesis: Some topics that I suggest need attention in pharmacovigilance are listed in Table 1. This list constitutes a partial agenda for studies on the language of pharmacovigilance.

Table 1. Linguistic topics relevant to pharmacovigilance
\begin{tabular}{|l|l|}
\hline Topic & Comments and examples \\
\hline $\begin{array}{l}\text { The use of appropriate technical terminology, } \\
\text { including avoidance of the use of terms that are } \\
\text { ambiguous or imprecise }\end{array}$ & $\begin{array}{l}\text { Misused words: "safety"; "idiosyncratic"; "adverse drug } \\
\text { event"; "indicates"; "syndrome"; "precision" }\end{array}$ \\
\hline $\begin{array}{l}\text { Avoidance of the use of colloquial terms at the } \\
\text { expense of accuracy }\end{array}$ & $\begin{array}{l}\text { Colloquial terms: "side effect"; "blood level"; "well } \\
\text { tolerated" }\end{array}$ \\
\hline $\begin{array}{l}\text { Avoidance of terms that involve extraordinary } \\
\text { meanings of ordinary words }\end{array}$ & Opaque terms: "designated medical event" \\
\hline $\begin{array}{l}\text { Using linguistic methods to formulate clear precise } \\
\text { definitions - shared understanding of what a term } \\
\text { currently means is essential }\end{array}$ & $\begin{array}{l}\text { (a) Etymology (but avoiding the etymological fallacy) } \\
\text { (b) General and specific usages } \\
\text { (c) An operational approach, taking into account what we } \\
\text { actually do or should do (implying that we can agree on } \\
\text { common practices) }\end{array}$ \\
\hline Coding of terms that describe adverse reactions & Dictionaries of adverse drug reactions \\
\hline $\begin{array}{l}\text { Clarification of the meanings of words when they are } \\
\text { translated into different languages }\end{array}$ & $\begin{array}{l}\text { Mistranslations (or none) and misspellings can impede } \\
\text { systematic reviews }\end{array}$ \\
\hline $\begin{array}{l}\text { The challenge of applying the technique of natural } \\
\text { language processing }\end{array}$ & $\begin{array}{l}\text { Most work is currently coming from Departments of } \\
\text { Biomedical Informatics and Biostatistics }\end{array}$ \\
\hline
\end{tabular}

\section{Teratogen surveillance in the 21 st century}

\author{
L.Yates ${ }^{1}$ \\ ${ }^{1}$ UK Teratology Information Service, Newcastle upon Tyne, UK
}

It is generally not considered ethical to undertake randomised controlled trials of pharmacological products in pregnant women. Identification of medicines that are harmful to the human fetus therefore relies almost exclusively on the analysis of observational data. Most established teratogen surveillance systems are based on the prospective follow up of a cohort of women who have used a particular medicine peri-conceptually or at some stage during pregnancy. Adverse pregnancy outcome rates can then be analysed and congenital malformations in offspring scrutinised for a pattern that may signal an embryopathy due to the exposure. However, use of prospective cohort methodology in current times is being challenged given the often high loss to follow-up rates, the low statistical power for these small cohorts and the inability of existing systems to detect adverse effects that manifest beyond birth. Novel pharmacovigilance methodologies are therefore being increasingly applied to surveillance of medicines used in pregnancy. Although offering promise for the future, careful interpretation and contextualisation of the results from analyses based on large electronic datasets and adverse event reporting systems is required. Whilst it is clear that modernisation of teratogen surveillance systems is needed, the value of more novel approaches over prospective cohorts remains to be demonstrated.

\section{Needles in hay-use of machine learning to identify references to drugs and adverse events in social media}

\section{N. Norén ${ }^{1}$}

Uppsala Monitoring Centre, Uppsala, Sweden

Background: Patient-generated data on the Internet can convey information that is relevant for pharmacovigilance. At a minimum, there are examples of posts in patient fora that provide as detailed information on individual cases as do some of the reports collected through the spontaneous reporting systems. In principle, these should be possible to identify and analyze for pharmacovigilance purposes. At the same time, there is much that we don't know: what drugs and adverse events tend to be discussed and where; what is the clinical value of these accounts; and can we effectively screen social media for such descriptions? In comparison 
with spontaneous reports and longitudinal electronic health records, an additional challenge in the analysis of social media is that relevant records must first be singled out from larger data sets where most items do not refer to drugs or medical events. What is more, references to drugs and medical events in these posts are not available in structured format but must be recognized from free text descriptions. Finally, implied adverse events must be distinguished from comments related to e.g. medical history and indications for treatment.

Objective: To develop and evaluate methods for identifying references to drugs and adverse events in social media, primarily Twitter.

Methods: As part of the WEB-RADR research collaboration, we developed machine learning methods to identify relevant search terms for social media posts related to specific medicines. We also developed named-entity recognition methods for drugs and medical events in free text [1-2], and predictive algorithms to distinguish adverse events from other medical events. Furthermore, we applied and evaluated disproportionality analysis of annotated social media items as a basis to detect emerging drug safety signals, in retrospective analyses using historical drug safety signals at the time of their identification as gold standard.

Results: Our results to date indicate that machine learning methods can help to identify and eliminate from search queries drug names with high levels of ambiguity and thereby reduce the number of irrelevant posts identified [1-2]. Similar methods can identify references to medical events in free text, but poor sensitivity is a real challenge [3] hampering downstream statistical signal detection [4].

Conclusions: Based on our findings thus far, we would recommend organizations with limited resources not to do statistical signal detection with current methods in Twitter or Facebook, at the expense of other pharmacovigilance activities.

Further sources of information/References:

1. Ellenius J, Bergvall T, Dasgupta N, Hedfors S, Pierce C, Norén GN. Medication Name Entity Recognition in Tweets Using Global Dictionary Lookup and Word Sense Disambiguation. Pharmacoepidemiol Drug Saf 2016; 25:414-5

2. Hedfors S, Bergvall T, Gilbert M, Pierce C, Dasgupta N, Ellenius J. Improving the Yield of Relevant Data for Pharmacovigilance Analysis by Reducing Search Term Complexity-A Study on Reddit Data. Abstract. Pharmacoepidemiol Drug Saf 2016; 25:412-3

3. Gattepaille L, Vidlin S, Pierce C, Bergvall T, Ellenius J. Detecting and encoding mentions of suspected Adverse Events in Twitter using Natural Language Processing. Abstract. To appear in Pharmacoepidemiol Drug Saf 2017

4. Caster O, Lerch M, Vroman B, van Stekelenborg J. Performance of Disproportionality Analysis for Statistical Signal Detection in Social Media Data. Abstract. Pharmacoepidemiol Drug Saf 2016; 25(S3): 411

\section{Pharmacovigilance of Anti-Cancer Medicines: Opportunities and Challenges}

\section{P. Pitts ${ }^{1}$}

Center for Medicine in the Public Interest, New York, USA

Background: More oncology medicines are being approved via expedited pathways that often require less-robust patient-level data and more aggressive post-marketing programs. The risk/benefit analyses of these medicines will be more regularly reviewed (post-approval) through still emerging risk evaluation and mitigation strategies.
Objective/Aim: To move beyond "expectedness" post-marketing theory to a more directive pre-approval program of "predictive pharmacovigilance," based on a more prospective and comprehensive program based on a sponsor's pre-market distribution and marketing strategies in order to to better predict real-world clinical adverse reactions.

Methods: Develop, in collaboration with global regulatory authorities (USFDA, EMA, Health Canada, ANVISA, Saudi FDA, Jordan FDA, China FDA, COFEPRIS, etc.) and the Council for International Organizations (CIOMS), more sensitive pre-market metrics of risk potential based on developer sales projections for both on and off-label indications. Based on these data sets, sponsor and agency would than develop a "Real World Pharmacovigilance Score" - a three-dimensional baseline prediction of likely adverse events based on projected volume and specific clinical use. This is of particular value for innovative, costly oncology medicines, since their use is, in the majority, of an off-label nature. It will be an equally valuable tool for new biologics as well as for better capturing the safety and efficacy of both biosimilars and generic non-biologic complex drugs (NBCDs). Predictive pharmacovigilance" is a tool for both clinical value and value-based reimbursement.

Results: The result of this program will be a design for validated tools and techniques for predictive pharmacovigilance design and implementation. Conclusion: Developing validated tools and techniques for predictive pharamcovigilance will assist all health systems in better understanding the risks and benefits of the medicines they regulate by understanding what should be happening once a new medicines moves from risk/benefit regulatory efficacy to real-world risk/effectiveness. This will be of particular utility for smaller regulatory agencies with fewer resources. By comparing pre-approval "predictive pharmacovigilance" data, developing regulatory authorities will be able to better understand the potential gap between what was predicted and what was actually measured (via more traditional pharmacovigilance methodologies). Predictive pharmacovigilance recognizes the value of understanding the imperfect reporting of real-world clinical use and that the absence of reporting is, in itself, an important post-marketing signal.

\section{Pharmacovigilance for herbal and traditional medicines: towards solutions and innovations}

\section{P. Routledge ${ }^{1}$}

University of Cardiff, Wales, UK

Herbal and traditional medicinal products are widely used, including by people with serious underlying conditions [1]. Like conventional medicines, they may produce pharmacological and sometimes also toxicological effects, resulting in adverse drug reactions (ADRs), including interactions with other concomitantly taken medications.

We examined the spontaneous suspected ADR reporting rates for herbal medicines to the UK Medicines and Healthcare Regulatory Products Agency (MHRA) via the Yellow Card Scheme for the ten-year period between 2006 and 2015. We also reviewed the scientific literature on pharmacovigilance of herbal and traditional medicines for the 25-year period between 1992 and 2017

The average annual number of yellow cards sent to MHRA over the 10 -year period was very low at 62 (range 45 to 94) with no discernible time-trend. The mean percentage of reports deemed to be "serious" was $73 \%$ (range 53 to 92 ) with the 4 lowest values over the most recent fouryear period. The mean percentage of reports provided by patients or their family members (as distinct from health professionals) was 44\% (range 29 to 72), with the highest value occurring in 2015 . 
In general, studies which measured the frequency of spontaneous reporting of suspected ADRs to herbal and traditional medicines showed low reporting rates and one UK survey indicated poor recognition and appreciation of the potential toxicity of herbal medicines by the public [2]. There is evidence that health professionals did not always ask about use of such medicines, and even when they did, they did not always record it [3]. There is a need for increased awareness by health professionals of the potential harms which can be associated with the use of herbal and traditional medicines, as well as for an improvement in the rate of reporting of suspected ADRs. This requires health professionals to comprehensively elicit and record a full medicines history (including the use of herbal, traditional and all over-the-counter medicines). The general public, patients and their families also need to be made aware that "natural" does not necessarily mean "safe" since in a survey published in $2009,22 \%$ of people who had used herbal medicines in the previous two years felt it was not necessary to inform their GP about use of such products [2].

Further sources of information/References

1. Damery, S, Gratus, C, Grieve, R, Warmington, S, Jones, J, Routledge, P, Greenfield, S, Dowswell, G, Sherriff, J, Wilson, S. The use of herbal medicines by people with cancer: a cross-sectional survey. Br J Cancer 2011; 104: 927-933

2. Ipsos Mori (2009) Public Perceptions of Herbal Medicines. https://www.ipsos.com/ipsos-mori/en-uk/public-perceptions-herbalmedicines

3. Skinner CM, Rangasami J. Preoperative use of herbal medicines: a patient survey. Br J Anaesth. 2002; 89: 792-5

\section{Regulatory implications of WEB-RADR findings}

\section{S. Brosch ${ }^{1}$}

European Medicines Agency, London, UK

Introduction: The use of tools and data sources such as social media provide a variety of public health opportunities but also raise new challenges that need to be addressed from a regulatory perspective. The Innovative Medicines Initiative (IMI) WEB-RADR project [1] has therefore set itself to discuss emerging regulatory questions.

Aim: To put forward recommendations that can inform the future development of good practice guides.

Methods: We performed a survey in 182 countries/jurisdictions to gather information on existing practices, guidance, and legal requirements on social media monitoring to identify potential safety issues related to medicines [2]. Focusing on challenges as part of the use of social media in pharmacovigilance, we collected questions from industry project partners based on their current experience and obtained feedback from healthcare professionals and patients in the frame of two WEB-RADR workshops. Additionally, we reviewed initial results of the WEB-RADR social media analytics and evaluation work streams to develop our recommendations. Results: The following six key topics emerged: 1 . Does the use of social media make a difference in pharmacovigilance obligations? 2. Do marketing authorisation holders (MAHs) need to screen social media for the purpose of signal detection? 3. What are the MAHs obligations to screen social media for collecting adverse drug reactions (ADRs)? 4. Should MAHs attempt to follow-up with social media users for the purpose of ADR reporting? 5. What constitutes a valid ADR report from social media? 6. What data protection and confidentiality aspects apply to the use of public social media data?

Our recommendations address these topics by reviewing the use of social media as a tool in performing pharmacovigilance activities (e.g. patient support programmes, risk minimisation activities), where we clarify reporting principles based on existing guidance. We further assessed the use as an additional data source to research content and activity on social media (e.g. signal detection) where we concluded that reporting principles based on secondary use of data should apply. Finally we summarised personal data protection requirements that should be adhered to.

Conclusions: We developed recommendations on the use of social media based on a risk based approach. This should allow for a better utilisation of this new data source but also ensure continuous monitoring of the safety of medicines and a timely identification of potential safety issues without overburdening the system.

Further sources of information/References:

1. Ghosh R and Lewis D. Aims and approaches of WEB-RADR: a consortium ensuring reliable ADR reporting via mobile devices and new insights from social media. Expert OpinDrug Saf 2015; 14:184553

2. Lengsavath M, Dal Pra A, de Ferran A, Brosch S, Harmark L, Newbould V, Goncalves S. Social Media Monitoring and Adverse Drug Reaction Reporting in Pharmacovigilance: An Overview of the Regulatory Landscape. Ther Innov Regul Sci 2017; 51:125-31

\section{Training Current and Future Drug Safety Specialists; Experience of running a Masters level course}

\section{S. Webley ${ }^{1}$}

University of Herts, Hertfordshire, UK

Background: Pharmacovigilance has gone through continuous change and development which has led to improvements in the way the safety of medicines is monitored and managed. Implementation of the new EU pharmacovigilance legislation in 2012, and the introduction of the European Medicines Agencies' (EMA) Good Pharmacovigilance Practices (GVP) modules, entailed much effort in the development of new procedures and practices. With all this comes a demand for high quality training and education. The University of Hertfordshire (UH), has been offering a high quality, fully validated, Higher Education credit rated, post-graduate programme in pharmacovigilance since 1996 in collaboration with the Pharmaceutical information and Pharmacovigilance Association (PIPA). Aim: The review set out to evaluate the UH programme from the 1996/97 academic year to 2016/17, looking specifically at course content, changes in student demographics, student progression through the programme and pharmaceutical industry support. The study also sought to identify good practice beneficial to future pharmacovigilance training and education.

Methods: A retrospective review of Pharmacovigilance Annual Monitoring and Evaluation Reports was conducted for the academic years between 1996/97 and 2016/17.

Results: Recruitment of students outside of the EU has increased from $8 \%$ to $25 \%$ over the review period. Similarly, the percentage of students from EU countries also increased from $18 \%$ to $30 \%$. The profile of students attending the course has changed; more experienced staff from larger companies tended to dominate the intake in the early years but not more recently. Regarding the types of companies sponsoring employees on the course; $64 \%$ of students were from large/medium size pharmaceutical companies in 1996/97 which fell to 40\% in 2012/13. There has been a rise (10\% to $36 \%$ over the period) in the number of contractors and provider companies sending employees onto the programme. There was a 2-fold increase in the number of students 
choosing to study the full Masters; before 2012-13 the majority of students $(79 \%)$ had enrolled to exit the programme with a post graduate diploma.

Conclusion: Over the past 20 years the pharmacovigilance Masters programme has kept abreast of the latest developments and regulatory changes within pharmacovigilance and moving pedagogical teaching methods, which has maintained the high quality of the programme. One key feature of the programme is the successful collaboration between regulatory bodies (EMA and NCAs), in addition to industry personnel who co-lead modules and/or facilitate teaching sessions. We believe this business facing approach and effective partnership is essential. In addition, working with other providers of pharmacovigilance education, such as the Eu2P programme, has enabled the $\mathrm{UH}$ programme to maintain its valuable role in training current and future drug safety specialists.

\section{When does Social Media add Value to Pharmacovigilance?}

\section{S. Maskell ${ }^{1}$ \\ ${ }^{1}$ University of Liverpool, Liverpool, UK}

Introduction: There are 100 million active Twitter users and 1.6 billion people using Facebook each day. Their social media posts could provide a rich source of data relevant to drug safety. Given the potential for significant reporting biases, it is unlikely that the demographic of side-effects reported in social media replicates the demographic found in spontaneous reporting.

Aim: To understand when social media can provide information that complements that derived from spontaneous reporting.

Methods: We assessed the utility of social media by analysing both the quantity of posts and the ability to process social media data to detect signals. In both contexts, we compared performance derived from data from March 2012 to March 2015. For social media, we used data provided by Epidemico's processing chain, as applied to Twitter and Facebook. For spontaneous reporting, we used Vigibase. For the analysis of quantity of posts, we derived estimates of the underlying rate of reporting for each of the MedDRA Preferred Terms (PTs) that Epidemico attempted to detect. This derivation made use of we used 194,496 posts that had been manually curated by Epidemico. We then compared the rate of reporting observed in Vigibase with the estimated underlying rate of reporting in social media. For the analysis of signal detection, we made use of a recently-developed reference standard. We compared performance achieved by: Vigibase; using a smaller number of higher-quality social media posts; using a larger number of lower-quality posts. For each data source, we calculated Receiver Operating Characteristic curves using Information Component (IC) as an exemplar signal detection algorithm.

Results: The total number of high-quality social media posts is approximately $10 \%$ of the number of spontaneous reports in the same time period. For some PTs (e.g., "cardiac arrest"), the estimated rate of reporting is substantially lower $(<0.1 \%)$. Despite this, there are certain PTs (e.g, "altered state of consciousness") for which the estimated underlying reporting rate is substantially higher in social media than in spontaneous reporting. With Epidemico's current processing chain, social media is less able to detect signals than spontaneous reporting, but detects different signals.

Discussion: Social media can provide information that complements that stored in spontaneous reporting databases. This implies that there is a need to develop algorithms for combining the information present in the two sources.

\section{Drug-induced liver injury}

\section{G. Aithal ${ }^{1}$} ${ }^{1}$ Nottingham Digestive Diseases Centre, University of Nottingham,
Nottingham, UK

Over 350 medications have been associated with adverse effects on the liver which is due probably to the central role of the liver in the metabolism and clearance of vast majority of drugs. There is a wide range of liver pathology where drug therapy has been implicated as an underlying aetiology. Drug-induced liver injury (DILI) is defined as an adverse hepatic reaction that is unexpected on the basis of the pharmacological action of the drug administered and it should be distinguished from the consequences of drug overdose. Recent large population based retrospective cohort studies and prospective case finding studies indicate a substantial difference between the incidence of DILI and the frequency with which on-going DILI is recognised or diagnosed. Over the past decade a number of drug-related factors such as daily dose, lipophilicity, hepatic metabolism and biliary excretion have been associated with increased DILI potential; consideration of these factors may assist in the identification of the offending drug in patients exposed to multiple medications. Investigations focused host genetics have identified specific HLA alleles as risk factors for DILI. These large genome-wide association studies have been instrumental in an improved understanding of the molecular mechanisms underlying the development of DILI highlighting a crucial role for the adaptive immune system in the pathogenesis of DILI. In addition, HLA alleles when used as tests have a high negative predictive value and therefore, have been used to rule out DILI due to specific drugs. Enhanced understanding of how HLA alleles contribute to injury risk is valuable for drug development. Translation of this research into effective pre-emption and primary prevention remains the goal.

\section{Precision dosing to reduce ADRs in children}

\section{Hawcutt ${ }^{1,2}$ \\ ${ }^{1}$ Department of Women's and Children's Health, University of Liverpool, Liverpool, United UK, ${ }^{2}$ NIHR Alder Hey Clinical Research Facility, UK}

Background: Severe cutaneous adverse reactions (SCAR) have in common that they are unpredictable, often dangerous and even life-threatening conditions leading to hospital admission or prolonging hospitalization. They may appear with blisters, pustules or systemic involvement.

Methods: Stevens-Johnson syndrome (SJS) and toxic epidermal necrolysis (TEN) are severe blistering muco-cutaneous conditions. They are considered a single disease entity of different severity-with SJS on the lower, TEN on the upper more severe end and SJS/TEN-overlap as the intermediate condition-sharing common features and risk factors. As an important differential diagnosis generalized bullous fixed drug eruption (GBFDE) has to be considered. Acute generalized exanthematous pustulosis (AGEP) is a pustular type of SCAR and drug reaction with eosinophilia and systemic symptoms (DRESS) is characterized by specific organ involvement. These reactions differ in clinical pattern, demographic data, prognosis and risk factors. These reactions are studied by the multinational RegiSCAR-project.

Results: Confirmed drug risk factors for SJS/TEN are allopurinol, antibacterial sulfonamides, certain antiepileptics (carbamazepine, lamotrigine, phenobarbital, phenytoin), nevirapine and non-steroidal 
inflammatory drugs of the oxicam-type. The time latency between beginning of drug use and onset of SJS/TEN varies from 4-28 days and usually represents the first continuous use of the drug. Examples for ongoing discussion are antipyretic, analgesic and secretolytic drugs, which are taken shortly before or after the onset of SJS/TEN. In GBFDE a previous reaction is common and the time between begin of drug use to reaction onset is very short (a few hours to a few days). Frequent inducers are sulfamethoxazole and metamizole.

Risk factors for AGEP are aminopenicillins and other antibiotics, but also antimyco- tics, (hydroxy-) chloroquine and diltiazem. The "time to onset" is short for most drugs (median of 2 days) and longer for some others (9-11 days). Allopurinol and carbamazepine have been identified as the most frequent inducers of DRESS in Europe (38\% of all cases) with a "time to onset" of several weeks, followed by lamotrigine and phenytoin. Conclusion: Ongoing surveillance is needed to identify cases of SCAR and validate the clinical diagnosis. The latter is of major importance as is the correct identification of the cause, both for appropriate treatment and for research including genetic investigations. New signals of potential risk factors have to be thoroughly evaluated, before wide-ranging decisions are made in the field of drug safety and pharmacovigilance. 


\section{AUTHOR INDEX}

A

Abadie, D 36

Abbas, A 251, 257

Abou Taam, M 78, 103, 104, 105, 106

Aboukhamis, R 200

Adams, A 97

Agbabiaka, T 32

Ahmad, T 160

Airaksinen, M 121

Ait Moussa, L 222

Aithal, G 281

Akici, A 244

Akrout, W 242

Aksoy, M 244

Al Ghufli, MM 203

Al-Aqqad, A 16

Al-Braik, FA 203

Aldea, A 162

Al-Dossari, M 16

Alfirevic, A 234, 238, 264

Alghasham, K 261

Ali, S 16

Aliat, Z 219, 222

Alj, L 144

Allen, C 138

Allouchery, M 77

Alshahrani, F 62

Alshammari, T 11

Altavilla, D 157

Alves, C 82

Al-zubiedi, S 128, 132

Ananzeh, A 16

Andrade, R 162, 163

Anghel, L 115

Anton, C 146, 160

Aoki, Y 86

Aouinti, I 174

Aparicio, T 76

Argentinis, E 20

Aristizabal, A 218

Arnaud, M 76, 85

Aronson, J 273

Arslan Unlu, Ez 252

Asfar, P 79

Aubert, L 235

Auffret, M 118

Auriche, P 171

Aydin, V 244

Azzouz, B 51, 78, 108, 235

B

Babai, S 228, 242

Backstrom, J 63

Badyal, K 146, 160

Bagheri, H 8, 36, 37, 38, 108

Bakkehøi, G 40

Ball, A 234

Baqar, J 16

Barbieri, M 140

Barcelos, F 182

Barillier, H 201

Barnes, J 35
Bassi, P 107

Batel Marques, F 82

Bauchau, V 164

Baumberger, M 18

Bawaresh, N 128

Bechar, H 144

Bellet, F. 154

Beloncle, F 79

Ben Salem, F 174, 185, 187, 202

Benabdellah, G 222

Béné, J 118

Benevent, J 5

Benkebil, M 152, 171

Bennani, I 253

Bensaid, D 148

Berdi, F 199

Bergvall, T 210

Bernier, C 108

Berthaut, V 19

Beyens, M.N 71, 154

Biasiotta, A 140

Bielen, C 4

Bird, H 225

Blanco, S 162

Blandizzi, C 96, 133, 135, 147

Bloch, KM 238

Blockman, M 197

Bollaerts, K 164

Bollegala, D 74

Bondon-Guitton, E 5

Bouhlel, M 185, 214

Boulle, A 197

Bourke, J 63

Bourneau Martin, D 79, 80, 108

Bousliman, Y 111, 112, 199, 246,247, 248, 249,

253

Bousquet, C 200

Bouterfa, F 6

Bradley, T 210

Branger, P 93

Bräutigam, K 158

Briet, M 79, 80, 108

Britton, S 168

Brody, R 72

Brooks, D 72

Brosch, S 278

Bryan, P 155, 161

Buclin, T 18, 19

Bucsa, C 53, 87

Bulik, N.B. 53,87

Burri, C 124, 125

Bwire, R. 63

C

Caduff-Janosa, P 86, 207

Calabro, M 134

Calvo, S 78

Capogrosso Sansone, A 96, 133, 135, 147

Capuano, A 167

Carey, E 97

Carlhant-Kowalski, D 42

Caron, G 117

Caro-Rojas, R 83

Carr, D 238
Carvajal, A 110, 141

Cassidy, C 97

Caster, O 48, 198

Castiella, A 162

Cauvin, JM 42, 119

Cavaco, A 121

Cave, A 31

Cazaubon, Y 51

Chaari, J 248, 250

Chadwick, A 234

Chan, K.A 268

Chandler, R 68

Chan-Liston, M 63

Charfi, O 174

Charfi, R 202, 214

Charfy, R 148

Chawadronow, V 50, 91, 92, 93

Chebane, L 5, 38

Chen, M 163

Chen, W 241

Chilloleau, A 56

Chipangura, P 114

Chopra, M 142

Chrétien, B 91, 92

Chu, CY 231

Chu, MP 241

Chung, S 52

Cicala, G 140

Claraz, P 76, 85

Cohen, K 197

Coles, M 266

Colin, AL 56

Comarova, N 6

Conde, I 162

Convertino, I 96, 133, 135, 147

Cooney, L 116

Coquerel, A 50

Corona, T 96, 135

Cortoos, A 129

Coulter, H 72

Cox, A.R 30, 62

Crepin, S 56

Crisafulli, C 134

Cueto-Sanchez, Al 163

Cutroneo, P.M 140, 167

Cuzin, L 37

D

D́Alessio, L 9

Da Cas, R 57, 58, 67, 145, 157

Daghfous, R 148, 174, 185, 187, 202

Damase-Michel, C 8

Damin-Pernik, M 71, 108, 154

Dardonville, Qu 36

Davies, A 237

Davies, M 7

De Ponti, F 57

De Smedt, T 164

Defer, G 93

Dekemp, J 118

Delgado, M 61

Delumeau, J-C 117

Derache, N 93

DeVore, L 130, 217 
Dey, S 46

Dhanda, S 101

Diacciati, S 135

Dickson, R 264

Djerada, Z 51

Do, Y 52

Dobrin, D 4

Dodoo, A 124, 125, 267

Doghmi, N 246

Donegan, K 138, 155, 164

Dong, C 35

Drablier, G 79, 80, 108

Dubuc-Lepetit, L 50

DURANTON, S 56

Durrieu, G 5, 8

Dussaye, A 146

$\mathbf{E}$

Easton, C 30

Eek, A 40

Eisinger, J 129

Ekhart, C. 26

Ekmekci, P.E 33, 34

El Aidli, S 185, 187

El Aridi, L 42, 119

El Fassi Fihri, A 144

EL HARRAK, M 112

El jebari, H 148, 202

El kassimi, Ilyas 248, 250

Elkarimi, E 144

Ellenius, J 95, 210

Engo, A 223

Engo Biongo, A 196, 215

Ennibi, K 248, 250

Eriksson, N 238

Errihani, H 144

Escobedo-Peña, J 204

Esseku, Y 267

Evans, A 7

Farcas, A 53, 87, 239

Farcaş, A 115

Fedrizzi, S 50, 91, 92, 93

Fermont, I.R 205

Fernandez, MC 162

Fernandez Acuña, JM 13, 28

Ferner, R 30, 146, 160

Ferrajolo, C 134, 167

Fettah, H 199

Figueiras, A 64

Finlay, A 72

Firenzuoli, F 67

Fokoue, A 46

Fourrier-Réglat, A 76, 85

Franceschi, MP 56

Freeman, J 63

French, S 237

Fujita, R 110

G

Gaboriau, L 108, 118

Gaies, E 148, 202

Galiulo, M 96, 133, 135, 147

Gallo, E 67

Gallo, J.C 24
Gama, S 48

Ganso, M 55

García Darderes, M 9, 24

García-Cortés, M 162

Garcia-Muñoz, B 162

Garcia-Serna, R 134

Garnica, S 218

Garza-Ocanas, L 204

Gattepaille, L 210

Gaudin, C 80

Gautier, S 118

Gavard, M 56

Géniaux, H 77

Getov, I 256

Ghattaoraya, G 264

Gines, P 162

Girardi, A 57

Glasgow, R 63

Golder, S 116

Gómez Acotto, C 9

Gomez-Dominguez, E 162

Gonzalez-Jimenez, A 162, 163

González-Nieto, C 204

Goodman, C 32

Goulenok, T 154

Gouverneur, A 76, 77, 85

Green, C 251, 257

Griffith, E 263

Grundmark, B 68, 90, 95, 210

Guchelaar, H.J 272

Gundert-Remy, U 158

Güner, M.D 33, 34

Guresci, N 189

Gursoz, H 244

Gwaza, L 114

H

Haaksman, M 66

Hajne, J 262, 263

Hall, V 122

Hallal, H 162

Happe, A 42, 119

Härmark, L 23, 35, 48, 68, 81, 109

Harmeyer, S 61

Harrison-Woolrych, M 35

Hartman, J 81, 207

Hasan, MY 203

Hauben, M 147, 213

Hawcutt, D 116, 282

Hazell, L 72

Heap, J 262, 263

Hegazy, A 229

Herdeiro, MT 64, 69, 70, 73

Herdeiro, T 184, 186

Hernández-Guerra, M 162

Herrera, L.G 122

Hewlett, D 61

Ho, J 153

Holder, G 129

Howard, J 159

Hsu, SH 231

$\mathrm{Hu}, \mathrm{J} 46$

Hugli, O 18

Hugman, B 21
Hult, S 210

Humbert, X 91

Hung, E 213

Hussain, T 11

I

Inacio, P 121

Isli, F 244

Issa, S 171

J

Jadeja, M 226, 236

Jang, B 52

Jantzem, H 42, 119

Jebabli, N 148, 202

Jimenez, M 162

Jones, A 262, 263, 264

Jones, J 197

Jorgensen, A 116

K

Kant, A. 22

Kant, A.C. 81

Karapentiaz, P 200

Kastalli, S 185, 187

Kaswa, M 223

Kennedy, J 153

Khattabi, A 144

KIM, H 52

Kisic, V 4

Klouz, A 202

Klouz, A 148

Knezevic, T 133, 147

Köberle, U 139, 158

Kowalski, D 119

L

Lacroix, I 8

Ladhari, C 187

Lagarce, L 79, 80, 108

Lane, S 39

Laroche, M.L 77

Lavi, T 143

Lavon, O 143, 205

Layton, D 27, 101

Lazarova, A 124, 125

Le Bellet, C 201

Le Guillou, C 42, 119

Le Louet, H 201, 228, 242

Lebanova, H 256

Lechheb, K 246, 248, 250, 253

Lefeuvre, C 56

Lemoine, M 200

Leprovost, D 200

Leucuţa, D 115

Lewis, D 270

Li, Y 20

Lillie, M 224

Lillo Le Louet, A 154, 200, 201

Lima-Dellamora, E.C 182

Livio, F 18, 19

Liwono Yana, J 151

Lizaroso-Soto, F.A 141

Loke, Y 116

Lord, R 266

Loughlin, L 265

Lucchetta, RC 186 
Lucena, M.I 161, 163

Lucht, F 154

Lula, Y 223

Luo, H 46

Lusakibanza, M 196

Lynn, E 39

Lyon, J 234

M

Macolic Sarinic, V 207

Maeder, M 124, 125

Mahalean, A 239

Mahlangu, G 114

Makhoul, H 143

Makram, S 111, 112

Maldonado Muete, C 83

Mampunza, S 215

Manso, G 36

Mantarro, S 96, 147

Manzano, DC 13

Manzon, C 19

Marchione, P 140

Marino, A 96, 133, 135, 147

Marley, S 27

Maroteau, C 238

Marotta, E 167

Marriott, J.F 62

Martin, Audrey 50

Mas, P 48

Maskell, S 74, 262, 263, 280

Mastroianni, PC 69, 70, 184, 186

Masuka, J.T 114

Matos, G 82

Maucorps, C 50, 91, 92, 93

Mazzanti, G 57, 58

Mazzei, T 135

Mbo, J 223

McEuen, K 163

McGettigan, P 31

Mecchio, A 157

Meddah, B 144

Medina-Caliz, I 162, 163

Meeraus, W 138

Mehta, U 197

Mendes, D 82

Menniti-Ippolito, F 57, 58, 67, 145, 157

Menon, K 129

Mensah Achampong, A 53

Mesia, G 223

Mesia Kahungu, G 215

Meskine, A 249

Mestres, J 134

Miglani, S 113

Migliardi, G 140

Miljkovic, M 4

Mini, E 135

Miremont-Salamé, G 76, 77, 85

Mockenhaupt, M 283

Mogosan, C 53

Moin, A 11

Moinuddin, K 16

Molimard, M 85

Molina Frojan, MB 13, 28

Monnier, A 80
Montane, E 162

Montastruc, F 5, 8

Montastruc, J.L 5, 8, 36, 37, 38

Morel, A 78, 235

Moreno, JM 162

Morillas, RM 162

Moro, P. 57

Morris, A 238

Moscol, S 110

Moss, J 116

Mouchantaf, R 6

Mouchel, C 56

Moulis, F 154

Moutaouakkil, Y 247

Mpiempe Ngamasata, T 215

Mrani Alaoui, A 246, 249, 253

Mullen, R 251

Muller, C 56

Mulongo, R 223

N

Naledi, T 197

Nascimento, LMF 73

Naser, A 221

Navarro, JM 162

Nchinech, N 111, 112, 199, 125

Niemcryk, S 63

Nobili, S 135

Noferi, LN 13, 28

Noize, P 76

Nootim, S 126

Norén, GN 210

Norén, N 275

Nsibu Ndosimao, C 196

Nsimba, G 223

Nyambayo, P 114

Nzolo Bomene, D 196, 215

O

Oh, S 52

O'Hara, A 264

Olivier, P 56

Olsson, S 124, 125

Olsson-Brown, A 266

Oniga, O 87

Oosterhuis, I 48

Oppamayun, Y 126

Oprean, R 115

Ortega, A 162

Osborne, V 101

Oscanoa, T 110, 141

Ouhaddouch, H 219, 222

Ouharakkat, Y 248

Ouk, T 56

Owen, R 168, 226

Öziş, S.E. 34

$\mathbf{P}$

Pachl, H 158

Pacurariu, A 31

Page, A 171

Palmer, C 238

Paret, N 108

Pariente, A 76, 77, 85, 99

Parikh, C 130

Parrilli, M 96, 135
Paso, F 13, 28

Pellegrino, N 167

Pelzer, L 22

Peng, M 63

Perault-Pochat, M.C 77

Pérez-Garza, A 204

Pérez-Rdz, A 204

Pérez-Rodríguez, E 204

Perino, J 77

Perkins, S 262, 263

Petitpain, N 56

Petracek, J 131

Peyro Saint Paul, L 56

Philibert, C 108

Phouthavong, O 37

Pieroni, S 133, 147

Pinar, O 189

Pirmohamed, M 74, 159, 237, 238, 262, 263, 264, 266

Pitts, P 276

Pitzer, M 139

Plueschke, K 31

Pokakul, P 126

Ponte, M 12, 13, 28

Ponte-Astoul, J 108

Popa, A 53

Poujol, P 154

Prause, L 139, 158

Prieto, M 162

Provoost, A 103

\section{R}

Radecka, A 265

Ragunathan, G 226

Randall, C 159

Rapin, S 71

Raschi, E 57

Raucci, U 145, 157

Reich, L 61

Renard, D 19

Ribeiro, MIB 73

RKIOUAK, A 250

Robles-Diaz, M 162, 163

Rocha, M 122

Roldan, E 24

Roldán, E 9

Rolfes, L 66

Roman, E 162

Romero-Gomez, M 162

Roque, F 64, 69, 70, 73, 184, 186

Rossi, R 145, 157

Rouillard, C 80

Rousseau, V 5, 36

Rousset, M 76, 85

Roussillon, C 56

Routledge, P 277

Roy, D 27

Ruellan, AL 154

Saad, RA 203

Sacco, J 266

Sadeghi, S 38

Sahnoun, R 187

Said, A 55 
Salameh, M 143

Salem, S 16

Salguiero, E 36

Salouage, Is 148, 202

Salvadori, S 147

Sanabria, J 162, 163

Sandberg, L 207

Sanjuán-Jiménez, R 162

Sardas, S 208

Sarrah, K 174

Sartori, D 90, 95, 124, 125, 210

Sassier, M 50

Savage, R 65

Schaller, MD 19

Schulz, M 55

Seabroke, S 155, 161

Sefiani, H 144, 219

Serra, A 12, 13

Serra, HA 28

Shakir, S 7, 27, 39, 72, 101

Sharma, L 224

Sheehan, P 63

Sheridan, J 35

Shi, M 129

Siah, S 249

Sihem, E.A 174

Simon, C 79

Singal, R 129

Sinha, I 116

Skalli, S 230

Slim, M 162, 163

Sloane, R 262, 263

Smaili, I 144

Smith, A 233

Sole, E 152, 171

Sommet, A 5, 8, 37

Sonsuphap, C 126

Soriano, G 162

Soulaymani, R 219, 222

Soulaymani Bencheikh, R 111, 112, 144, 199 , 230, 247

Spina, E 140, 167

Stammschulte, T 139, 158

Star, K 68, 86

Stenberg-Nilsen, H 40

Stephens, C 163

Stewart, A 197

Stoev, S 256
Strøm, B.O. 40

Sukasem, C 269

Sultana, J 134

Suzuki, A 163

T

Taavola, H 48, 68

Tabba, A 248

Tadlaoui, Y 112

Tadlaoui, Y 253

Talibi, I 144

Tashtoush, MM 203

Tassi, R 135

Tatley, M 65

Tebaa, A 111, 112, 144, 199, 219, 222, 247,

250,253

Thomas, A 97

Thompson, A 159

Timár-Horváth, K 44, 45

Titievsky, L 164

Tona, G 223

Tona Lutete, G 196, 215

Tosi, EJ 13

Trabelsi, S 148, 202

Trad, L 111

Tregunno, P 48

Trenque, T 51, 78, 103, 104, 105, 235

Trifiro, G 134, 140, 167, 271

Trinh, N 152

Trombotto, V 186

Trotta, F 157

Tuccori, M 96, 133, 135, 147

$\mathbf{U}$

Unal, B 189, 208, 252

Upton, J 266

Urich, M 124, 125

V

Valnet Rabier, M.B 108

Van geertruyden, J.P 196, 215

Van Hunsel, F 22, 23

Van Vynckt, J 103

Vannacci, A 96, 135

Varallo, FR 69, 70, 184, 186

Vaughan, S 168, 233

Velez-Nandayapa, L 129

Velez-Nandayapa, L 130

Veyrac, G 108

Viard, A 71

Vidlin, S 210
Vignale, JF 13, 28

Vitalone, A 58

Voget, J 197

Vohra, S 35

Voirol, P 19

Vorstenbosch, S 29

van Balveren-Slingerland, L 29

van Boekel, A 109

van Eijk, M 198

van Hunsel, F 26, 66, 68

van Puijenbroek, E 26, 29, 66, 109

W

Wadelius, M 238

Wambura, C 127

Wang, Fe 20

Wang, Qi 221

Wang, W 61

Watson, S 68, 86

Webley, S 279

Wei, L 221

Weits, G 81

Westerberg, C 90

White, B 221

Whitehead, J 153

Wider, B 32

Wong, I 221

Wong, J 155

Wong, L 221

WOO, Y 52

Woode, E 267

Wu, W.L 241

$\mathbf{X}$

Xiao, 20

Y

Yates, L 274

Younus, M 61

$\mathbf{Z}$

Zack, L 143

Zaiem, A 174, 185, 187

Zapata, E 162

Zbir, A 248

Zgolli, F 174

Zhang, P 46

Zhou, J 20

Zielinski, LR 28

Ziraoui, O 246, 249, 253

Zweigenbaum, P 200 